\title{
Synthesis and Structural Revision of Glyphaeaside C
}

\author{
Brendan J. Byatt, ${ }^{\dagger}$ Atsushi Kato ${ }^{\star}$ Stephen G. Pyne ${ }^{* \dagger}$ \\ †School of Chemistry and Molecular Bioscience, University of Wollongong, Wollongong, New South Wales 2522, Australia \\ tDepartment of Hospital Pharmacy, University of Toyama, Sugitani, Toyama 2630, Japan
}

\section{Supporting Information}

NMR Data Comparison of Natural Glyphaeaside C with Structurally Related Piperidine Iminosugars........... S2

NMR Data Comparison of Natural Glyphaeaside C with Structurally Related Pyrrolidine Iminosugars .......... S4

NMR Data Comparison of Natural Glyphaeaside C with 2a·TFA and 2b·TFA.......................................... S6

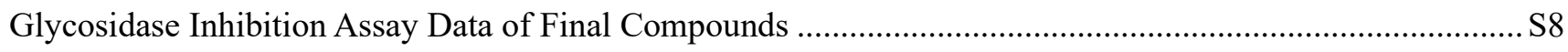

Proposed Alternative Structures of A- and B-Type Glyphaeasides........................................................... S9

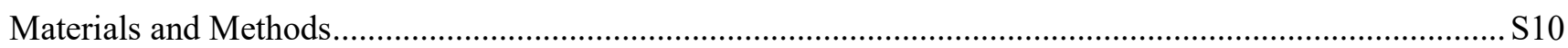

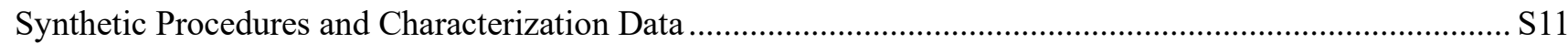

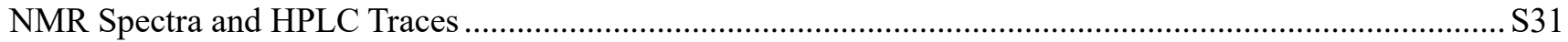

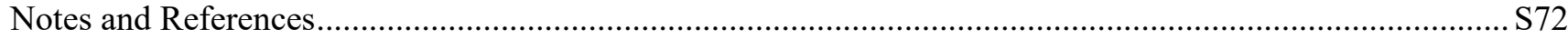




\section{NMR Data Comparison of Natural Glyphaeaside C with Structurally Related Piperidine Iminosugars}

All NMR spectra were measured in $\mathrm{CD}_{3} \mathrm{OD}$. Chemical shifts are reported as per their publication, noting the reference value used if stated. Coupling constant $(J)$ values are reported in Hz. Compound atom numbering as per natural glyphaeaside $\mathrm{C}$ in its original publication (1), with other related compounds adjusted to match.

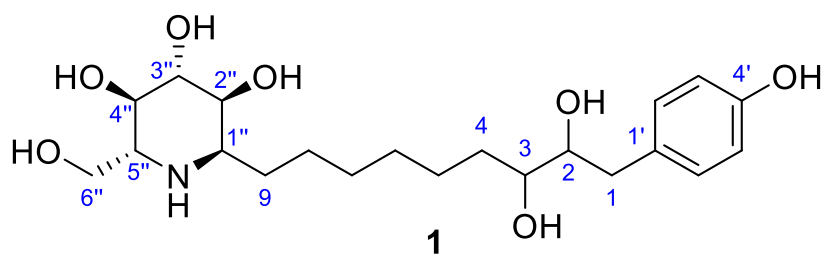<smiles>CCCCCCCC[C@H]1N[C@@H](CO)[C@@H](O)[C@H](O)[C@H]1O</smiles>

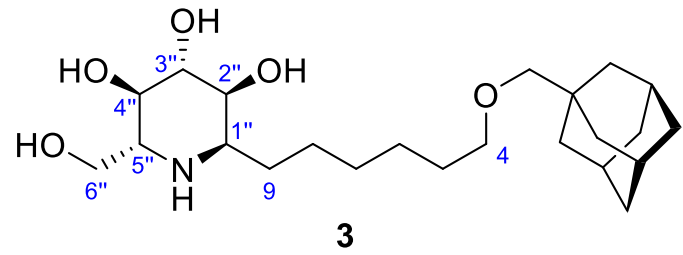

Table S1: Comparison of assigned ${ }^{1} \mathrm{H}$ NMR spectroscopic data for natural glyphaeaside $\mathrm{C}$ with related iminosugars $\mathbf{S 1}$ and $\mathbf{3} \cdot \mathrm{HCl}$.

\begin{tabular}{|c|c|c|c|c|c|}
\hline \multirow[b]{2}{*}{ Proton } & \multirow{2}{*}{$\frac{\text { Glyphaeaside C }^{\text {(natural) }}{ }^{1, a}}{\delta_{\mathrm{H}}}$} & \multicolumn{2}{|c|}{$\mathbf{S} 1^{2, b}$} & \multicolumn{2}{|c|}{$3 \cdot \mathrm{HCl}^{3, c}$} \\
\hline & & $\delta_{\mathrm{H}}$ & $\Delta \delta \mathrm{H}$ & $\delta_{\mathrm{H}}$ & $\Delta \delta_{\mathrm{H}}$ \\
\hline $1 "$ & $3.88(\mathrm{~m})$ & $\begin{array}{l}2.93(\mathrm{ddd}, J= \\
11.0,5.5,3.2)\end{array}$ & -0.95 & $\begin{array}{c}3.46(\operatorname{td}, J=6.9 \\
3.6)\end{array}$ & -0.42 \\
\hline $2^{\prime \prime}$ & $3.40(\mathrm{dd}, J=6.7,2.5)$ & $\begin{array}{l}3.51(\mathrm{dd}, J= \\
9.6,5.5)\end{array}$ & +0.11 & $\begin{array}{c}3.78(\mathrm{dd}, J= \\
\quad 6.6,3.6)\end{array}$ & +0.38 \\
\hline $3 "$ & $4.25(\mathrm{t}, J=6.7)$ & $\begin{array}{l}3.35(\mathrm{dd}, J= \\
9.6,8.7)\end{array}$ & -0.90 & $\begin{array}{c}3.71(\mathrm{dd}, J= \\
6.7,6.6)\end{array}$ & -0.54 \\
\hline $4 "$ & $3.99(\mathrm{dd}, J=7.5,6.7)$ & $\begin{array}{l}3.01(\mathrm{dd}, J= \\
9.6,8.7)\end{array}$ & -0.98 & $\begin{array}{c}3.62(\mathrm{dd}, J= \\
6.7,6.7)\end{array}$ & -0.37 \\
\hline $5^{\prime \prime}$ & $3.41(\mathrm{~m})$ & $\begin{array}{c}2.63(\mathrm{ddd}, J= \\
9.6,7.8,3.2)\end{array}$ & -0.78 & $3.34-3.32(\mathrm{~m})$ & -0.08 \\
\hline \multirow[t]{2}{*}{$6^{\prime \prime}$} & $3.80(\mathrm{dd}, J=12.1,5.2)$ & $\begin{array}{c}3.39(\mathrm{dd}, J= \\
11.0,7.8)\end{array}$ & -0.41 & $\begin{array}{c}3.83(\mathrm{dd}, J= \\
12.0,3.8)\end{array}$ & +0.03 \\
\hline & $3.86(\mathrm{dd}, J=12.1,3.5)$ & $\begin{array}{c}3.81(\mathrm{dd}, J= \\
11.0,3.2)\end{array}$ & -0.05 & $\begin{array}{c}4.00(\mathrm{dd}, J= \\
12.0,7.1)\end{array}$ & +0.14 \\
\hline \multirow[t]{2}{*}{9} & $1.64(\mathrm{~m})$ & $1.47-1.20(\mathrm{~m})$ & $-d$ & $1.65-1.60(\mathrm{~m})$ & -0.02 \\
\hline & & $1.56(\mathrm{~m})$ & +0.08 & $1.98-1.90(\mathrm{~m})$ & - \\
\hline \multirow[t]{2}{*}{8} & $1.37(\mathrm{~m})$ & $1.47-1.20(\mathrm{~m})$ & - & $1.50-1.44(\mathrm{~m})$ & +0.12 \\
\hline & $1.50(\mathrm{~m})$ & & & & -0.03 \\
\hline
\end{tabular}


${ }^{a}{ }^{1} \mathrm{H}$ NMR spectrum referenced to $\mathrm{CHD}_{2} \mathrm{OD}$ at $\delta=3.33 \mathrm{ppm} .{ }^{b}{ }^{1} \mathrm{H}$ NMR spectrum reference point not stated. ${ }^{c}$ Presumed to be $\mathrm{HCl}$ salt by contrast of spectral data with $\mathbf{S 1}$, and since the compound was not eluted from an ion-exchange resin with $\mathrm{NH}_{4} \mathrm{OH}$, although it was not reported as such in the original publication. ${ }^{1} \mathrm{H}$ NMR spectrum referenced to the deuterated solvent, exact value not stated. ${ }^{d}$ Overlapping peaks, difference from glyphaeaside $\mathrm{C}$ not calculated.

Table S2: Comparison of assigned ${ }^{13} \mathrm{C}$ NMR spectroscopic data for natural glyphaeaside $\mathrm{C}$ with related iminosugars $\mathbf{S 1}$ and $\mathbf{3} \cdot \mathrm{HCl}$.

\begin{tabular}{|c|c|c|c|c|c|}
\hline \multirow[b]{2}{*}{ carbon } & \multirow{2}{*}{$\frac{\text { Glyphaeaside } \mathbf{C} \text { (natural) }^{1, a}}{\delta_{\mathrm{C}}}$} & \multicolumn{2}{|c|}{$\mathbf{S} 1^{2, b}$} & \multicolumn{2}{|c|}{ 3. $\mathrm{HCl}^{3, c}$} \\
\hline & & $\delta_{\mathrm{C}}$ & $\Delta \delta_{\mathrm{C}}$ & $\delta_{\mathrm{C}}$ & $\Delta \delta_{\mathrm{C}}$ \\
\hline $1^{\prime \prime}$ & 70.1 & 57.5 & -12.6 & 55.3 & -14.8 \\
\hline $2^{\prime \prime}$ & 66.0 & 74.3 & +8.3 & 70.4 & +4.4 \\
\hline $3 "$ & 75.1 & 76.1 & +1.0 & 72.8 & -2.3 \\
\hline $4 "$ & 76.7 & 74.3 & -2.4 & 69.5 & -7.2 \\
\hline $5^{\prime \prime}$ & 64.8 & 56.1 & -8.7 & 59.5 & -5.3 \\
\hline $6^{\prime \prime}$ & 58.6 & 63.9 & +5.3 & 58.6 & 0 \\
\hline 9 & 34.6 & $33.1-14.4^{d}$ & - & 27.6 & -7.0 \\
\hline 8 & 26.7 & $33.1-14.4^{d}$ & - & 27.2 & -0.5 \\
\hline
\end{tabular}

${ }^{a 13} \mathrm{C}$ NMR spectrum referenced to $\mathrm{CD}_{3} \mathrm{OD}$ at $\delta=49 \mathrm{ppm} .{ }^{b}{ }^{13} \mathrm{C}$ NMR spectrum reference point not stated. ${ }^{c}{ }^{13} \mathrm{C}$ NMR spectrum referenced to the deuterated solvent, exact value not stated. ${ }^{d}$ Side chain ${ }^{13} \mathrm{C}$ NMR resonances listed and not assigned, difference from glyphaeaside $\mathrm{C}$ not calculated. 


\section{NMR Data Comparison of Natural Glyphaeaside C with Structurally Related Pyrrolidine Iminosugars}

All NMR spectra were measured in $\mathrm{CD}_{3} \mathrm{OD}$. Chemical shifts are reported as per their publication, noting the reference value used if stated. Coupling constant $(J)$ values are reported in Hz. Compound atom numbering as per natural glyphaeaside $\mathrm{C}$ in its original publication (1), with other related compounds adjusted to match.
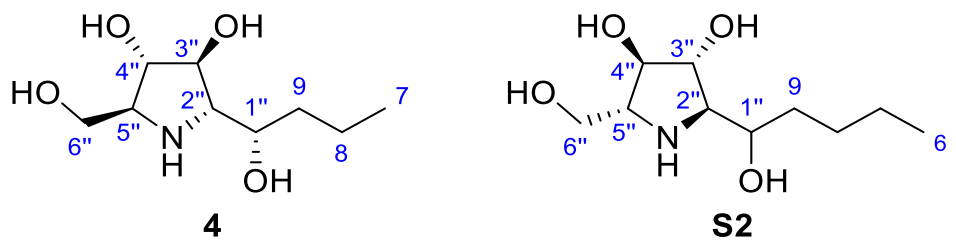

Table S3: Comparison of assigned ${ }^{1} \mathrm{H}$ NMR spectroscopic data for natural glyphaeaside $\mathrm{C}$ with related iminosugars $\mathbf{4} \cdot \mathrm{HCl}$ and $\mathbf{S 2}$.

\begin{tabular}{|c|c|c|c|c|c|}
\hline \multirow[b]{2}{*}{ proton } & \multirow{2}{*}{$\frac{{\text { Glyphaeaside } \mathbf{C} \text { (natural) }^{1, a}}_{\delta_{\mathrm{H}}}}{\delta}$} & \multicolumn{2}{|c|}{ 4. $\mathrm{HCl}^{4, b, c}$} & \multicolumn{2}{|c|}{$\mathbf{S} 2^{5, b, d}$} \\
\hline & & $\delta_{\mathrm{H}}$ & $\Delta \delta_{\mathrm{H}}$ & $\delta_{\mathrm{H}}$ & $\Delta \delta_{\mathrm{H}}$ \\
\hline $1^{\prime \prime}$ & $3.88(\mathrm{~m})$ & $3.90-3.77(\mathrm{~m})$ & $-e$ & $\begin{array}{c}3.63(\mathrm{dt}, J=8.3 \\
4.2)\end{array}$ & -0.25 \\
\hline $2^{\prime \prime}$ & $3.40(\mathrm{dd}, J=6.7,2.5)$ & $3.40-3.37(\mathrm{~m})$ & - & $\begin{array}{c}3.05(\mathrm{dd}, J= \\
6.8,4.2)\end{array}$ & -0.35 \\
\hline $3 "$ & $4.25(\mathrm{t}, J=6.7)$ & $4.23(\mathrm{t}, J=6.8)$ & -0.02 & $\begin{array}{c}4.13(\mathrm{dd}, J= \\
6.8,4.9)\end{array}$ & -0.12 \\
\hline $4^{\prime \prime}$ & $3.99(\mathrm{dd}, J=7.5,6.7)$ & $3.97(\mathrm{t}, J=6.8)$ & -0.02 & $\begin{array}{c}4.07(\mathrm{dd}, J= \\
4.9,4.2)\end{array}$ & +0.08 \\
\hline $5^{\prime \prime}$ & $3.41(\mathrm{~m})$ & $3.40-3.37(\mathrm{~m})$ & - & $\begin{array}{c}3.25(\mathrm{ddd}, J= \\
6.4,5.9,4.2)\end{array}$ & -0.16 \\
\hline \multirow[t]{2}{*}{$6^{\prime \prime}$} & $3.80(\mathrm{dd}, J=12.1,5.2)$ & $3.90-3.77(\mathrm{~m})$ & - & $\begin{array}{c}3.68(\mathrm{dd}, J= \\
11.0,6.4)\end{array}$ & -0.12 \\
\hline & $3.86(\mathrm{dd}, J=12.1,3.5)$ & $3.90-3.77(\mathrm{~m})$ & - & $\begin{array}{c}3.78(\mathrm{dd}, J= \\
11.0,5.9)\end{array}$ & -0.08 \\
\hline 9 & $1.64(\mathrm{~m})$ & $1.64-1.36(\mathrm{~m})$ & - & $1.66-1.48(\mathrm{~m})$ & - \\
\hline \multirow[t]{2}{*}{8} & $1.37(\mathrm{~m})$ & $1.64-1.36(\mathrm{~m})$ & - & $1.43-1.30(\mathrm{~m})$ & - \\
\hline & $1.50(\mathrm{~m})$ & & & $1.66-1.48(\mathrm{~m})$ & - \\
\hline
\end{tabular}

\footnotetext{
${ }^{a}{ }^{1} \mathrm{H}$ NMR spectrum referenced to $\mathrm{CHD}_{2} \mathrm{OD}$ at $\delta=3.33 \mathrm{ppm} .{ }^{b}{ }^{1} \mathrm{H}$ NMR spectrum referenced to tetramethylsilane as the internal standard. ${ }^{c}$ Presumed to be $\mathrm{HCl}$ salt as the compound was not eluted from an ion-exchange resin with $\mathrm{NH}_{4} \mathrm{OH}$, although it was not reported as such in the original publication. All NMR spectroscopic data listed and not assigned in publication, assigned in table based on similarity with the natural product. ${ }^{d}$ Relative configuration at C1" could not be determined from the NMR spectral data. ${ }^{e}$ Overlapping peaks, difference from glyphaeaside $\mathrm{C}$ not calculated.
} 
Table S4: Comparison of assigned ${ }^{13} \mathrm{C}$ NMR spectroscopic data for natural glyphaeaside $\mathrm{C}$ with related iminosugars $\mathbf{4} \cdot \mathrm{HCl}$ and $\mathbf{S 2}$.

\begin{tabular}{cccccc} 
& \multicolumn{2}{c}{ Glyphaeaside C (natural) (1,a $^{1,}$} & \multicolumn{2}{c}{$\mathbf{4 . H C l}^{4, b, c}$} & \multicolumn{2}{c}{$\mathbf{S 2}^{5, b}$} \\
\cline { 2 - 6 } carbon & $\delta \mathrm{c}$ & $\delta \mathrm{c}$ & $\Delta \delta \mathrm{c}$ & $\delta \mathrm{c}$ & $\Delta \delta_{\mathrm{c}}$ \\
$1^{\prime \prime}$ & 70.1 & 69.7 & -0.4 & 75.2 & +5.1 \\
$2^{\prime \prime}$ & 66.0 & 66.0 & 0 & 68.7 & +2.7 \\
$3^{\prime \prime}$ & 75.1 & 75.1 & 0 & 75.8 & +0.7 \\
$4^{\prime \prime}$ & 76.7 & 76.6 & -0.1 & 76.1 & -0.6 \\
$5^{\prime \prime}$ & 64.8 & 64.8 & 0 & 64.4 & -0.4 \\
$6^{\prime \prime}$ & 58.6 & 58.6 & 0 & 64.0 & +5.4 \\
9 & 34.6 & 36.7 & +2.1 & 36.7 & +2.1 \\
8 & 26.7 & 20.0 & -6.7 & 31.2 & +4.5 \\
\hline
\end{tabular}

${ }^{a}{ }^{13} \mathrm{C}$ NMR spectrum referenced to $\mathrm{CD}_{3} \mathrm{OD}$ at $\delta=49 \mathrm{ppm} .{ }^{b{ }^{13} \mathrm{C}}$ NMR spectrum referenced to tetramethylsilane as the internal standard. ${ }^{c}$ All NMR spectroscopic data listed and not assigned in publication, assigned in table based on similarity with glyphaeaside $\mathrm{C}$. 


\section{NMR Data Comparison of Natural Glyphaeaside C with 2a・TFA and $2 \mathrm{~b} \cdot$ TFA.}

All NMR spectra were measured in $\mathrm{CD}_{3} \mathrm{OD}$. Chemical shifts are reported as per their publication, noting the reference value used if stated. Coupling constant $(J)$ values are reported in Hz. Compound atom numbering as per natural glyphaeaside $\mathrm{C}$ in its original publication (1), with other related compounds adjusted to match.

Table S5: Comparison of assigned ${ }^{1} \mathrm{H}$ NMR spectroscopic data for natural glyphaeaside $\mathrm{C}$ with iminosugars $\mathbf{2 a} \cdot$ TFA and $\mathbf{2} \mathbf{b} \cdot$ TFA.

\begin{tabular}{|c|c|c|c|c|c|}
\hline \multirow[b]{2}{*}{ proton } & \multirow{2}{*}{$\frac{\text { Glyphaeaside } \mathbf{C} \text { (natural) }^{1, a}}{\delta_{\mathrm{H}}}$} & \multicolumn{2}{|c|}{$\mathbf{2 a} \cdot \mathrm{TFA}^{a}$} & \multicolumn{2}{|c|}{$\mathbf{2 b} \cdot \mathrm{TFA}^{a}$} \\
\hline & & $\delta \mathrm{H}$ & $\Delta \delta_{\mathrm{H}}$ & $\delta_{\mathrm{H}}$ & $\Delta \delta_{\mathrm{H}}$ \\
\hline $1^{\prime \prime}$ & $3.88(\mathrm{~m})$ & $3.92-3.83(\mathrm{~m})$ & $-b$ & $3.92-3.83(\mathrm{~m})$ & - \\
\hline $2^{\prime \prime}$ & $3.40(\mathrm{dd}, J=6.7,2.5)$ & $3.45-3.38(\mathrm{~m})$ & - & $3.45-3.38(\mathrm{~m})$ & - \\
\hline $3^{\prime \prime}$ & $4.25(\mathrm{t}, J=6.7)$ & $4.26(\mathrm{t}, J=7.0)$ & +0.01 & $4.26(\mathrm{t}, J=7.0)$ & 0 \\
\hline $4 "$ & $3.99(\mathrm{dd}, J=7.5,6.7)$ & $\begin{array}{c}3.99(\mathrm{dd}, J= \\
8.5,6.6)\end{array}$ & 0 & $\begin{array}{c}3.99(\mathrm{dd}, J= \\
8.5,6.6)\end{array}$ & 0 \\
\hline $5^{\prime \prime}$ & $3.41(\mathrm{~m})$ & $3.45-3.38(\mathrm{~m})$ & - & $3.45-3.38(\mathrm{~m})$ & - \\
\hline \multirow[t]{2}{*}{$6^{\prime \prime}$} & $3.80(\mathrm{dd}, J=12.1,5.2)$ & $\begin{array}{c}3.80(\mathrm{dd}, J= \\
12.0,5.2)\end{array}$ & 0 & $\begin{array}{c}3.80(\mathrm{dd}, J= \\
12.0,5.2)\end{array}$ & 0 \\
\hline & $3.86(\mathrm{dd}, J=12.1,3.5)$ & $3.92-3.83(\mathrm{~m})$ & - & $3.92-3.83(\mathrm{~m})$ & - \\
\hline 9 & $1.64(\mathrm{~m})$ & $1.69-1.58(\mathrm{~m})$ & -0.01 & $1.67-1.59(\mathrm{~m})$ & -0.01 \\
\hline \multirow[t]{2}{*}{8} & $1.37(\mathrm{~m})$ & $1.58-1.27(\mathrm{~m})$ & - & $1.58-1.29(\mathrm{~m})$ & - \\
\hline & $1.50(\mathrm{~m})$ & & & & \\
\hline 7 & $1.31(\mathrm{~m})$ & $1.58-1.27(\mathrm{~m})$ & - & $1.58-1.29(\mathrm{~m})$ & - \\
\hline 6 & $1.37(\mathrm{~m})$ & $1.58-1.27(\mathrm{~m})$ & - & $1.58-1.29(\mathrm{~m})$ & - \\
\hline \multirow[t]{2}{*}{5} & $1.37(\mathrm{~m})$ & $1.58-1.27(\mathrm{~m})$ & - & $1.58-1.29(\mathrm{~m})$ & - \\
\hline & $1.50(\mathrm{~m})$ & & & & \\
\hline 4 & $1.53(\mathrm{~m})$ & $1.58-1.27(\mathrm{~m})$ & - & $1.58-1.29(\mathrm{~m})$ & - \\
\hline 3 & $3.41(\mathrm{~m})$ & $3.45-3.38(\mathrm{~m})$ & - & $3.45-3.38(\mathrm{~m})$ & - \\
\hline 2 & $3.59(\mathrm{ddd}, J=8.5,5.5,3.4)$ & $\begin{array}{c}3.59(\mathrm{ddd}, J= \\
8.5,5.4,3.4)\end{array}$ & 0 & $\begin{array}{c}3.59(\mathrm{ddd}, J= \\
8.5,5.4,3.4)\end{array}$ & 0 \\
\hline \multirow[t]{2}{*}{1} & $2.64(\mathrm{dd}, J=13.8,8.0)$ & $\begin{array}{c}2.64(\mathrm{dd}, J= \\
13.7,8.0)\end{array}$ & 0 & $\begin{array}{c}2.64(\mathrm{dd}, J= \\
13.8,8.0)\end{array}$ & 0 \\
\hline & $2.80(\mathrm{dd}, J=13.8,5.5)$ & $\begin{array}{c}2.80(\mathrm{dd}, J= \\
13.7,5.4)\end{array}$ & 0 & $\begin{array}{c}2.80(\mathrm{dd}, J= \\
13.7,5.4)\end{array}$ & 0 \\
\hline $2^{\prime}, 6^{\prime}$ & $7.09(\mathrm{dd}, J=6.4,2.1)$ & $7.08(\mathrm{~d}, J=8.5)$ & -0.01 & $7.08(\mathrm{~d}, J=8.5)$ & -0.01 \\
\hline $3^{\prime}, 5^{\prime}$ & $6.72(\mathrm{dd}, J=6.4,2.1)$ & $6.72(\mathrm{~d}, J=8.6)$ & 0 & $6.72(\mathrm{~d}, J=8.5)$ & 0 \\
\hline
\end{tabular}

\footnotetext{
${ }^{a}{ }^{1} \mathrm{H}$ NMR spectrum referenced to $\mathrm{CHD}_{2} \mathrm{OD}$ at $\delta=3.33 \mathrm{ppm} .{ }^{b}$ Overlapping peaks, difference from glyphaeaside $\mathrm{C}$ not calculated.
} 
Table S6: Comparison of assigned ${ }^{13} \mathrm{C}$ NMR spectroscopic data for natural glyphaeaside $\mathrm{C}$ with iminosugars $\mathbf{2} \mathbf{a} \cdot$ TFA and $\mathbf{2 b} \cdot$ TFA.

\begin{tabular}{|c|c|c|c|c|c|}
\hline \multirow[b]{2}{*}{ carbon } & \multirow{2}{*}{$\frac{{\text { Glyphaeaside } \mathbf{C}\left(\text { (natural }^{1, a}\right.}^{1, a}}{\delta_{\mathrm{C}}}$} & \multicolumn{2}{|c|}{ 2a.TFA ${ }^{a}$} & \multicolumn{2}{|c|}{ 2b. TFA $^{a}$} \\
\hline & & $\delta_{\mathrm{C}}$ & $\Delta \delta_{\mathrm{C}}$ & $\delta_{\mathrm{C}}$ & $\Delta \delta_{\mathrm{C}}$ \\
\hline $1^{\prime \prime}$ & 70.1 & 70.0 & -0.1 & 70.0 & -0.1 \\
\hline $2^{\prime \prime}$ & 66.0 & 66.0 & 0 & 66.0 & 0 \\
\hline $3^{\prime \prime}$ & 75.1 & 75.1 & 0 & 75.1 & 0 \\
\hline $4^{\prime \prime}$ & 76.7 & 76.7 & 0 & 76.7 & 0 \\
\hline $5^{\prime \prime}$ & 64.8 & 64.8 & 0 & 64.8 & 0 \\
\hline $6^{\prime \prime}$ & 58.6 & 58.5 & -0.1 & 58.6 & 0 \\
\hline 9 & 34.6 & 34.6 & 0 & 34.6 & 0 \\
\hline 8 & 26.7 & $26.7^{b}$ & 0 & $26.7^{b}$ & 0 \\
\hline 7 & 30.6 & $30.6^{b}$ & 0 & $30.6^{b}$ & 0 \\
\hline 6 & 30.5 & $30.5^{b}$ & 0 & $30.5^{b}$ & 0 \\
\hline 5 & 27.0 & $27.0^{b}$ & 0 & $27.0^{b}$ & 0 \\
\hline 4 & 34.2 & 34.2 & 0 & 34.2 & 0 \\
\hline 3 & 74.0 & 74.0 & 0 & 74.0 & 0 \\
\hline 2 & 76.6 & 76.5 & -0.1 & 76.5 & -0.1 \\
\hline 1 & 39.7 & 39.8 & +0.1 & 39.7 & 0 \\
\hline $1^{\prime}$ & 131.4 & $\mathrm{nd}^{c}$ & - & 131.4 & 0 \\
\hline $2^{\prime}, 6^{\prime}$ & 131.4 & 131.4 & 0 & 131.4 & 0 \\
\hline $3^{\prime}, 5^{\prime}$ & 116.1 & 116.1 & 0 & 116.1 & 0 \\
\hline $4^{\prime}$ & 156.6 & 156.7 & +0.1 & 156.7 & +0.1 \\
\hline
\end{tabular}

${ }^{a}{ }^{13} \mathrm{C}$ NMR spectrum referenced to $\mathrm{CD}_{3} \mathrm{OD} \delta=49 \mathrm{ppm} .{ }^{b}$ Assignments made by comparison with natural product, unable to distinguish using available 2D NMR data. ${ }^{c}$ Not detected by DEPTQ or HMBC. 


\section{Glycosidase Inhibition Assay Data of Final Compounds}

Table S7: Concentration of iminosugars giving 50\% inhibition of various glycosidases.

\begin{tabular}{|c|c|c|c|c|c|}
\hline \multirow[b]{2}{*}{ enzyme } & \multicolumn{5}{|c|}{$\mathrm{IC}_{50}(\mu \mathrm{M})$} \\
\hline & $\begin{array}{c}\text { Glyphaeaside C } \\
\text { (natural) }^{1}\end{array}$ & $\mathbf{2 a}$ & $2 \mathbf{b}$ & $2 \mathbf{a}^{\prime}$ & $2 \mathbf{b}^{\prime}$ \\
\hline \multicolumn{6}{|l|}{$\alpha$-glucosidase } \\
\hline rice & $13 \%^{a}$ & $21.3 \%^{b}$ & $5.7 \%^{b}$ & $3.0 \%^{b}$ & $4.2 \%^{b}$ \\
\hline yeast & $-c$ & $2.3 \%^{b}$ & $1.7 \%^{b}$ & $1.4 \%^{b}$ & $0.9 \%^{b}$ \\
\hline \multicolumn{6}{|l|}{$\beta$-glucosidase } \\
\hline almond & 0.15 & 0.92 & 0.77 & 0.99 & 0.82 \\
\hline bovine liver & - & 0.037 & 0.019 & 0.060 & 0.038 \\
\hline human lysosome & - & 33 & 195 & 36 & 55 \\
\hline \multicolumn{6}{|l|}{$\alpha$-galactosidase } \\
\hline \multicolumn{6}{|l|}{$\beta$-galactosidase } \\
\hline bovine liver & - & 0.031 & 0.020 & 0.043 & 0.019 \\
\hline A. oryzae & $82 \%^{a}$ & - & - & - & - \\
\hline $\begin{array}{l}\boldsymbol{\alpha} \text {-mannosidase } \\
\text { jack bean }\end{array}$ & - & $4.8 \%^{b}$ & $\mathrm{NI}^{e}$ & $\mathrm{NI}^{e}$ & $\mathrm{NI}^{e}$ \\
\hline \multicolumn{6}{|l|}{$\beta$-mannosidase } \\
\hline snail & 4.5 & 48 & 30 & 56 & 35 \\
\hline $\begin{array}{l}\alpha \text {-L-fucosidase } \\
\text { bovine kidney }\end{array}$ & $\mathrm{NI}^{e}$ & $9.5 \%^{b}$ & $2.0 \%^{b}$ & $3.6 \%^{b}$ & $\mathrm{NI}^{e}$ \\
\hline \multicolumn{6}{|l|}{ trehalase } \\
\hline porcine kidney & - & $10.7 \%^{b}$ & $2.3 \%^{b}$ & $\mathrm{NI}^{e}$ & $\mathrm{NI}^{e}$ \\
\hline \multicolumn{6}{|l|}{ amyloglucosidase } \\
\hline A. niger & - & 17 & 14 & 30 & 26 \\
\hline \multicolumn{6}{|l|}{$\alpha$-L-rhamnosidase } \\
\hline \multicolumn{6}{|l|}{$\beta$-glucuronidase } \\
\hline E. coli & - & 110 & 573 & 428 & 181 \\
\hline bovine liver & - & $14.9 \%^{b}$ & $1.0 \%^{b}$ & $\mathrm{NI}^{e}$ & $\mathrm{NI}^{e}$ \\
\hline
\end{tabular}

\footnotetext{
${ }^{a}$ Inhibition $\%$ at $1000 \mu \mathrm{M} .{ }^{b}$ Inhibition $\%$ at $100 \mu \mathrm{M} .{ }^{c}$ Not tested. ${ }^{d}$ No inhibition at $1000 \mu \mathrm{M} .{ }^{e}$ No inhibition at $100 \mu \mathrm{M}$.
} 


\section{Proposed Alternative Structures of A- and B-Type Glyphaeasides}

The same rationale that led to our reassignment of glyphaeaside $\mathrm{C}$ is also applicable to the other glyphaeasides. Rearranging the B-type glyphaeasides would suggest a shared 2,5-dideoxy-2,5-imino-D-altritol iminosugar core (or the L-enantiomer), which is itself a natural product isolated from the roots of Adenophora triphylla, given the abbreviation "DIA" (Figure S1). ${ }^{6}$ By the same convention, the A-type glyphaeasides would be derivatives of 1,2,5-trideoxy-2,5-imino-D-altritol (or the L-enantiomer) - another natural product, isolated from the bark of Angylocalyx pynaertii. ${ }^{7}$ This deoxyiminosugar could be considered "1-deoxyDIA", although such abbreviation was not used in the isolation paper ( $\mathrm{C} 1$ of a 2,5-iminohexitol corresponds to $\mathrm{C} 6$ of a 2,5-disubstituted pyrrolidine - see 6-deoxy-DMDP ${ }^{7}$ - though for broussonetines this position is usually considered a hydroxymethyl substituent and thus not numbered).
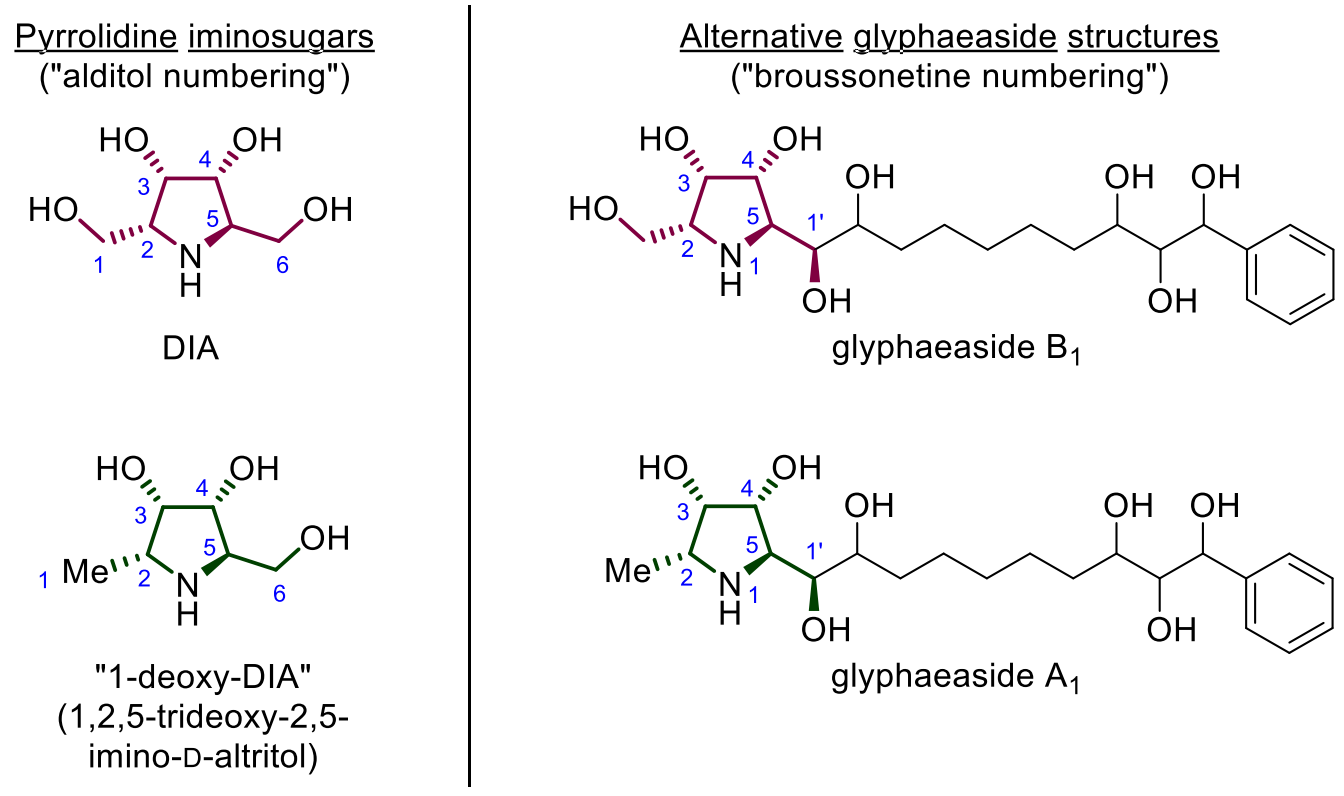

Figure S1. Proposed alternative structures of glyphaeasides $A_{1}$ and $B_{1}$ and related pyrrolidine iminosugars.

Unlike the structures that were originally proposed for the A- and B-type glyphaeaside (tentatively given L-DFJ and D-DGJ configurations, respectively, based on literature precedent), there is no reason to expect their revised structures to possess opposing core configurations - in fact, the precedent offered from the literature would suggest they have the same absolute configuration, differing only by their methyl/hydroxymethyl substituent at $\mathrm{C} 2$. 


\section{Materials and Methods}

All reagents were obtained from commercial suppliers and used without further purification unless stated otherwise. Anhydrous THF was distilled from Na/benzophenone prior to use. Anhydrous DCM was distilled from $\mathrm{CaH}_{2}$ prior to use. All other anhydrous solvents were stored over $4 \AA$ molecular sieves for at least $2 \mathrm{~d}$ prior to use. Reactions involving anhydrous solvents were carried out using oven-dried and desiccator-cooled glassware and performed under a positive pressure of $\mathrm{N}_{2}$ unless stated otherwise. Reactions conducted above room temperature were performed in a heated oil bath. Where indicated, solutions were concentrated by evaporation using a rotary evaporator at reduced pressure. Column chromatography was carried out on silica gel $60(40-63 \mu \mathrm{m})$ under a positive pressure of compressed air. Analytical TLC was performed on aluminum plates pre-coated with silica gel 60 F254 or reversed-phase (RP-18) modified silica gel 60 F254 and visualized with $254 \mathrm{~nm}$ light and immersion in ceric ammonium molybdate stain solution followed by heating. Analytical HPLC was performed on a Shimadzu Prominence system (LC-2030C 3D) using a Phenomenex Gemini C18 $5 \mu$ column $(2.0 \times 100 \mathrm{~mm})$ with a binary solvent system (solvent A: water, $\mathrm{pH} 2.4$, with $0.0025 \%$ TFA; solvent $\mathrm{B}: \mathrm{MeOH}$ ), a combination isocratic/gradient elution program, and a flow rate of $0.2 \mathrm{~mL} / \mathrm{min}$, and the chromatogram was monitored at $200 \mathrm{~nm}$. Semi-preparative HPLC was performed using a Shimadzu Prominence solvent delivery unit (LC-20AP) equipped with a low-pressure gradient unit and a Phenomenex Gemini C18 $5 \mu$ column $(10.0 \times 150 \mathrm{~mm})$, using the same solvent composition and adjusted elution programs that were used under analytical conditions. Samples purified by semi-preparative HPLC were filtered through a $0.45 \mu$ m nylon filter, dissolved in $100 \%$ solvent A (ca. 0.5 $\mathrm{mL}$ ), injected in $80 \mu \mathrm{L}$ portions, collected manually by chromatogram observation, and concentrated. Optical rotation values were measured using a Jasco P-2000 polarimeter with a $10 \mathrm{~cm}, 2 \mathrm{~mL}$ cell. Infrared spectral data were determined on neat samples using a Bruker Vertex 70 FT-IR spectrometer. NMR spectra were recorded using a Bruker Avance III 400 spectrometer $\left(400.16 \mathrm{MHz},{ }^{1} \mathrm{H} ; 100.63 \mathrm{MHz},{ }^{13} \mathrm{C} ; 376.49\right.$ $\left.\mathrm{MHz},{ }^{19} \mathrm{~F}\right)$ with a BBO Observe probe and Bruker Avance Neo 500 spectrometer $\left(499.75 \mathrm{MHz},{ }^{1} \mathrm{H} ; 125.67\right.$ $\mathrm{MHz},{ }^{13} \mathrm{C}$ ) with a BBO Prodigy $\mathrm{N}_{2}$ cryoprobe. Chemical shifts $(\delta)$ are given in ppm relative to residual solvent signals $\left(\mathrm{CD}_{2} \mathrm{HOD} \delta=3.33 \mathrm{ppm}\right.$, DMSO- $d 5 \delta=2.50 \mathrm{ppm}$, pyridine- $\left.d 4 \delta=8.71 \mathrm{ppm}\right)$ or an internal reference $\left(\mathrm{TMS} \delta=0.00 \mathrm{ppm}\right.$ for $\left.\mathrm{CDCl}_{3}\right)$ for ${ }^{1} \mathrm{H}$ NMR spectra, deuterated solvent signals $\left(\mathrm{CDCl}_{3} \delta=77.16\right.$ ppm, $\mathrm{CD}_{3} \mathrm{OD} \delta=49.00 \mathrm{ppm}$, DMSO- $d 6 \delta=39.52 \mathrm{ppm}$, pyridine- $d 5 \delta=150.35 \mathrm{ppm}$ ) for ${ }^{13} \mathrm{C}$ NMR spectra, and an external reference $\left(\mathrm{C}_{6} \mathrm{H}_{5} \mathrm{CF}_{3}\right.$ in $\mathrm{CDCl}_{3} \delta=-62.74$ for $\mathrm{CD}_{3} \mathrm{OD}$, pyridine- $\left.d 5\right)$ for ${ }^{19} \mathrm{~F}$ NMR spectra. NMR assignments were made using DEPTQ, COSY, HSQC, and HMBC experiments. Concerning the ${ }^{1} \mathrm{H}$ NMR spectroscopic data for compounds isolated as mixtures of isomers, the isomeric ratio is noted, and the integrations for non-overlapping protons of each isomer are divided accordingly. For compounds existing as unequal mixtures of rotamers, the ${ }^{1} \mathrm{H}$ NMR and ${ }^{13} \mathrm{C}$ NMR chemical shifts of the minor rotamer are denoted with an asterisk. High-resolution mass spectra were obtained using a Waters Xevo QToF spectrometer $(\mathrm{ESI}+)$ and are reported as $(\mathrm{m} / \mathrm{z})$. 


\section{Synthetic Procedures and Characterization Data}

\section{$\underline{2,4,5-\text { Triiodo- } 1 H \text {-imidazole }(\mathbf{S 3})^{8}}$}

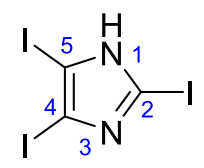

S3

To a round-bottom flask containing hexane $(300 \mathrm{~mL})$ was added iodine $(15.2 \mathrm{~g}, 60.0 \mathrm{mmol})$. The mixture was stirred at $\mathrm{rt}$ for $30 \mathrm{~min}$, followed by the addition of water $(300 \mathrm{~mL})$ and imidazole $(1.36 \mathrm{~g}, 20.0 \mathrm{mmol})$. The reaction mixture was stirred at $\mathrm{rt}$ for $24 \mathrm{~h}$, adding portions of a $\mathrm{NaOH}$ solution $(8 \% \mathrm{w} / \mathrm{v})$ when precipitation was observed. The reaction mixture was filtered, and the hexane layer was removed by decantation. The aqueous layer was acidified ( $\mathrm{pH} 5)$ using conc. $\mathrm{HCl}(32 \%)$, and the resulting precipitate was collected by vacuum filtration. The crude material was dissolved in hot $\mathrm{MeOH}(25 \mathrm{~mL})$, seeded with a small crystal of imidazole, and cooled to rt. Once crystallization was observed, the flask containing the product was cooled to $-20{ }^{\circ} \mathrm{C}$ in a freezer and stood overnight. The crystals were collected by vacuum filtration (4.47 $\mathrm{g}$ ), and the filtrate was concentrated until further precipitation was observed on cooling. The flask was stood at $\mathrm{rt}$ for $2 \mathrm{~h}$, and the resulting crystals were collected by vacuum filtration and combined with the first crop, affording $\mathbf{S 3}$ (6.48 g, $14.5 \mathrm{mmol}$ ) in $73 \%$ yield as a yellow crystalline solid. The analytical data were in agreement with the literature. , $^{8}$

Mp $182-184{ }^{\circ} \mathrm{C}\left(\right.$ lit. $\left.{ }^{8} \mathrm{mp} 190-192{ }^{\circ} \mathrm{C}\right)$.

IR (neat) $\tilde{v}_{\max }\left(\mathrm{cm}^{-1}\right): 2549,1806,1505,1412,1371,1267,1162,969$.

${ }^{1} \mathrm{H}$ NMR (400.16 MHz, $\left.\left(\mathrm{CD}_{3}\right)_{2} \mathrm{SO}\right): \delta 13.35$ (br, $\left.1 \mathrm{H}, \mathrm{NH}\right)$.

${ }^{13} \mathrm{C}$ NMR $\left(100.63 \mathrm{MHz},\left(\mathrm{CD}_{3}\right)_{2} \mathrm{SO}\right): \delta 89.8(3 \times \mathrm{ArC})$.

$\underline{(2 R, 3 S, 4 S)-2,3,5-T r i s\left(\text { benzyloxy)-4-iodopentanal } O \text {-(tert-butyldiphenylsilyl) oxime }(\mathbf{S 4})^{9}\right.}$

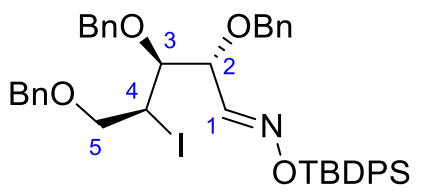

(E)-S4

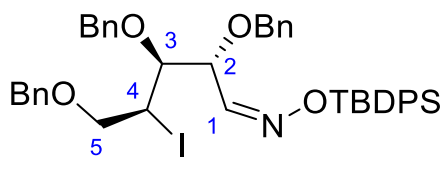

(Z)-S4

To a two-neck round-bottom flask containing anhydrous $\mathrm{MeOH}(22 \mathrm{~mL})$ at $0{ }^{\circ} \mathrm{C}$ (ice bath) under a flow of $\mathrm{N}_{2}$ was added in portions sodium metal $(1.56 \mathrm{~g}, 68.0 \mathrm{mmol})$. Once the solid had completely reacted, the 
$\mathrm{NaOMe}$ solution was warmed to $\mathrm{rt}$ and added to a solution of 2,3,5-tri- $O$-benzyl- $\beta$-D-arabinofuranose (7.14 $\mathrm{g}, 17.0 \mathrm{mmol})$ and hydroxylamine hydrochloride $(9.45 \mathrm{~g}, 136 \mathrm{mmol})$ in anhydrous $\mathrm{MeOH}(85 \mathrm{~mL})$. The reaction mixture was stirred at $\mathrm{rt}$ for $2 \mathrm{~h}$ and concentrated. The residue was dissolved in $\mathrm{CH}_{2} \mathrm{Cl}_{2}(100 \mathrm{~mL})$ and washed with water $(3 \times 70 \mathrm{~mL})$. The organic layer was dried over $\mathrm{MgSO}_{4}$, filtered, and concentrated. The residue was dissolved in anhydrous $\mathrm{CH}_{2} \mathrm{Cl}_{2}(22 \mathrm{~mL})$, followed by the addition of imidazole $(1.50 \mathrm{~g}$, $22.1 \mathrm{mmol})$. Once the solid had completely dissolved, tert-butyldiphenylsilyl chloride $(5.50 \mathrm{~mL}, 21.3$ $\mathrm{mmol}$ ) was added carefully. The reaction mixture was stirred at $\mathrm{rt}$ for $30 \mathrm{~min}$, diluted with $\mathrm{CH}_{2} \mathrm{Cl}_{2}(35 \mathrm{~mL})$, and washed with water $(35 \mathrm{~mL})$. The aqueous layer was extracted with $\mathrm{CH}_{2} \mathrm{Cl}_{2}(3 \times 70 \mathrm{~mL})$, and the combined organic layers were washed with sat. aq. $\mathrm{NaCl}(35 \mathrm{~mL})$, dried over $\mathrm{MgSO}_{4}$, filtered, and concentrated. The residue was dissolved in anhydrous toluene $(425 \mathrm{~mL})$, followed by the addition of $\mathbf{S 3}(6.60 \mathrm{~g}, 14.8$ $\mathrm{mmol})$ and triphenylphosphine $(8.92 \mathrm{~g}, 34.0 \mathrm{mmol})$. The reaction mixture was heated at reflux, stirred for $1 \mathrm{~h}$, and cooled to rt. Additional $\mathbf{S 3}$ (3.33 g, $7.48 \mathrm{mmol}$ ) and triphenylphosphine (4.46 g, $17.0 \mathrm{mmol})$ were added, and the reaction mixture was heated at reflux, stirred for $4 \mathrm{~h}$, cooled to $\mathrm{rt}$, and quenched with sat. aq. $\mathrm{NaHCO}_{3}(140 \mathrm{~mL})$. Crystals of iodine were added in portions with stirring until the organic layer no longer became turbid upon addition. Excess iodine was quenched with sat. aq. $\mathrm{Na}_{2} \mathrm{~S}_{2} \mathrm{O}_{3}(140 \mathrm{~mL})$. The aqueous layer was extracted with EtOAc $(140 \mathrm{~mL})$, and the combined organic layers were washed with sat. aq. $\mathrm{NaCl}$ $(75 \mathrm{~mL})$, dried over $\mathrm{MgSO}_{4}$, filtered, and concentrated. The residue was suspended in 1:1 Et $2 \mathrm{O} /$ pentane $(250 \mathrm{~mL})$ to precipitate triphenylphosphine oxide, which was removed by vacuum filtration. The filtrate was concentrated, and the residue was purified by column chromatography (10:90 EtOAc/hexane), affording (E/Z)-S4 (9.69 g, $12.4 \mathrm{mmol}, 3.4: 1)$ in 73\% yield as a colorless oil. Qualitatively pure fractions of individual isomers were used for analytical data characterization, which were in agreement with the literature. $^{9-11}$

\section{$\underline{(E)-\mathbf{S 4}}$}

$R_{\mathrm{f}}: 0.50$ (10:90 EtOAc/hexane).

$[\alpha]_{\mathrm{D}}^{25}+22.1\left(c 1.01, \mathrm{CHCl}_{3}\right)\left[\mathrm{lit}^{10}[\alpha]_{\mathrm{D}}^{25}-27.6\left(c 1.05, \mathrm{CHCl}_{3}\right)\right.$, lit. $\left.{ }^{11}[\alpha]_{\mathrm{D}}^{24}+16.2\left(c 1.39, \mathrm{CHCl}_{3}\right)\right]$.

IR (neat) $\tilde{v}_{\max }\left(\mathrm{cm}^{-1}\right): 3069,3030,2930,2857,1454,1361,1113,1071,916,822,735,695,613$.

${ }^{1} \mathrm{H}$ NMR (499.75 MHz, $\mathrm{CDCl}_{3}$ ): $\delta$ 7.76-7.69 (m, 4 H, 4× $\left.\mathrm{ArCH} \mathrm{SiPh}_{2}\right), 7.59$ (d, J=8.1 Hz, $1 \mathrm{H}, \mathrm{H1}$ ), $7.43-$ $7.14\left(\mathrm{~m}, 21 \mathrm{H}, 21 \times \mathrm{ArCH} \mathrm{OBn} / \mathrm{SiPh}_{2}\right), 4.89$ (d, $\left.J=11.5 \mathrm{~Hz}, 1 \mathrm{H}, \mathrm{CHHOBn}\right), 4.69$ (d, $J=11.5 \mathrm{~Hz}, 1 \mathrm{H}$, $\mathrm{CH} H \mathrm{OBn}$ ), 4.47 (d $J=11.6 \mathrm{~Hz}, 1 \mathrm{H}, \mathrm{CH} \mathrm{HOBn}), 4.34-4.23$ (m, $\left.4 \mathrm{H}, \mathrm{CH} H \mathrm{OBn}, \mathrm{CH}_{2} \mathrm{OBn}, \mathrm{H} 2\right), 4.13$ (ddd, $J=8.6,5.3,3.0 \mathrm{~Hz}, 1 \mathrm{H}, \mathrm{H} 4), 3.72$ (dd, $J=10.1,8.6 \mathrm{~Hz}, 1 \mathrm{H}, \mathrm{CHH} \mathrm{H}$ ), 3.59 (dd, $J=10.1,5.3 \mathrm{~Hz}, 1 \mathrm{H}$, $\mathrm{CH} H \mathrm{H} 5), 3.52$ (dd, $J=7.2,3.0 \mathrm{~Hz}, 1 \mathrm{H}, \mathrm{H} 3), 1.14$ (s, $\left.9 \mathrm{H}, \mathrm{Me}_{3} \mathrm{C}\right)$. 
${ }^{13} \mathrm{C}$ NMR (125.67 MHz, $\left.\mathrm{CDCl}_{3}\right): \delta 153.0(\mathrm{C} 1), 138.5,137.8,137.6(3 \times \mathrm{ArC}), 135.72,135.70(2 \times \mathrm{ArCH})$, $133.5,133.3$ (2×ArC), 129.9, 128.6, 128.5, 128.4, 128.2, 128.0, 127.94, 127.86, 127.81, 127.76, 127.7 (23× $\mathrm{ArCH}), 81.1(\mathrm{C} 2), 77.6(\mathrm{C} 3), 74.9\left(\mathrm{CH}_{2} \mathrm{OBn}\right), 72.9,72.7\left(\mathrm{C} 5, \mathrm{CH}_{2} \mathrm{OBn}\right), 71.5\left(\mathrm{CH}_{2} \mathrm{OBn}\right), 31.4(\mathrm{C} 4)$, $27.3\left(\mathrm{Me}_{3} \mathrm{C}\right), 19.5\left(\mathrm{Me}_{3} \mathrm{C}\right){ }^{12}$

LRMS (ESI) m/z: $[\mathrm{M}+\mathrm{Na}]^{+} 806(100 \%)$.

$\underline{(Z)-\mathbf{S 4}}$

$R_{\mathrm{f}:} 0.45$ (10:90 EtOAc/hexane).

$[\alpha]_{\mathrm{D}}^{25}-14.7\left(c 1.17, \mathrm{CHCl}_{3}\right)\left[\operatorname{lit}^{10}[\alpha]_{\mathrm{D}}^{25}-25.4\left(c 1.5, \mathrm{CH}_{2} \mathrm{Cl}_{2}\right)\right]$

IR (neat) $\tilde{v}_{\max }\left(\mathrm{cm}^{-1}\right): 3069,3020,2929,2856,1454,1361,1109,1067,912,820,737,695,606$.

${ }^{1} \mathrm{H}$ NMR (499.75 MHz, $\left.\mathrm{CDCl}_{3}\right): \delta$ 7.75-7.67 (m, 4 H, 4× $\left.\mathrm{ArCH} \mathrm{SiPh}_{2}\right), 7.46-7.21(\mathrm{~m}, 21 \mathrm{H}, 21 \times \mathrm{ArCH} \mathrm{OBn} /$ $\left.\mathrm{SiPh}_{2}\right), 7.04(\mathrm{~d}, J=6.6 \mathrm{~Hz}, 1 \mathrm{H}, \mathrm{H} 1), 5.35(\mathrm{dd}, J=6.6,5.1 \mathrm{~Hz}, 1 \mathrm{H}, \mathrm{H} 2), 4.69\left(\mathrm{ABq}, \Delta \delta_{\mathrm{AB}}=0.03, J_{\mathrm{AB}}=\right.$ $11.3 \mathrm{~Hz}, 2 \mathrm{H}, \mathrm{CH}_{2} \mathrm{OBn}$ ), 4.59 (d, $J=11.6 \mathrm{~Hz}, 1 \mathrm{H}, \mathrm{CHHOBn}$ ), 4.47-4.30 (m, $4 \mathrm{H}, \mathrm{CH} H \mathrm{OBn}, \mathrm{CH}_{2} \mathrm{OBn}$, H4), 3.84 (t, $J=4.9 \mathrm{~Hz}, 1 \mathrm{H}, \mathrm{H} 3$ ), 3.73 (dd, $J=10.7,6.6 \mathrm{~Hz}, 1 \mathrm{H}, \mathrm{CHH} \mathrm{H5),} 3.64$ (dd, $J=10.7,6.0 \mathrm{~Hz}, 1$ $\mathrm{H}, \mathrm{CH} H \mathrm{H} 5), 1.13$ (s, $9 \mathrm{H}, 3 \times \mathrm{Me}_{3} \mathrm{C}$ ).

${ }^{13} \mathrm{C}$ NMR (125.67 MHz, $\left.\mathrm{CDCl}_{3}\right): \delta 155.1$ (C1), 138.0, 137.8, 137.3 (3× $\left.\mathrm{ArC}\right), 135.7(\mathrm{ArCH}), 134.9(\mathrm{ArCH})$, 133.15, 133.12 (2×ArC), 130.0, 129.8, 128.6, 128.54, 128.45, 128.14, 128.12, 128.0, 127.92, 127.86, 127.8 $(23 \times \mathrm{ArCH}), 79.3(\mathrm{C} 3), 74.9\left(\mathrm{CH}_{2} \mathrm{OBn}\right), 73.7(\mathrm{C} 2), 73.0\left(\mathrm{CH}_{2} \mathrm{OBn}\right), 72.7(\mathrm{C} 5), 72.5\left(\mathrm{CH}_{2} \mathrm{OBn}\right), 31.3(\mathrm{C} 4)$, $27.3\left(3 \times \mathrm{Me}_{3} \mathrm{C}\right), 19.5\left(\mathrm{Me}_{3} \mathrm{C}\right), 19.2$.

LRMS (ESI) m/z: [M+ Na $]^{+} 806(100 \%)$.

$(2 R, 3 R, 4 R)-3,4-B i s\left(\right.$ benzyloxy)-2-((benzyloxy)methyl)-3,4-dihydro- $2 H$-pyrrole-1-oxide (S5) ${ }^{11}$

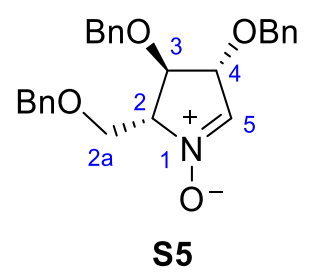

To a solution of $(E / Z)-\mathbf{S} 4(6.65 \mathrm{~g}, 8.49 \mathrm{mmol}, 3.4: 1)$ in anh. THF $(45 \mathrm{~mL})$ was added a $1.0 \mathrm{M}$ solution of $n$ tetrabutylammonium fluoride in THF $(8.80 \mathrm{~mL}, 8.80 \mathrm{mmol})$. The reaction mixture was stirred at $\mathrm{rt}$ for 16 $\mathrm{h}$ and concentrated. The residue was purified by column chromatography (40:60 $\rightarrow$ 100:0 EtOAc/hexane), 
affording S5 (1.88 g, $4.51 \mathrm{mmol})$ in 53\% yield as a yellow solid. The analytical data were in agreement with the literature. ${ }^{10,11}$

$R_{\mathrm{f}}: 0.30$ (70:30 EtOAc/hexane).

$[\alpha]_{\mathrm{D}}^{25}-23.7\left(c 0.90, \mathrm{CHCl}_{3}\right)\left[\operatorname{lit}^{10}[\alpha]_{\mathrm{D}}^{25}-41\left(c 1, \mathrm{CHCl}_{3}\right)\right.$, lit. $\left.^{11}[\alpha]_{\mathrm{D}}^{19}-41.7\left(c 1.00, \mathrm{CHCl}_{3}\right)\right]$.

IR (neat) $\tilde{v}_{\max }\left(\mathrm{cm}^{-1}\right): 3048,2922,2870,1591,1495,1451,1361,1275,1087,1076,734,697$.

${ }^{1} \mathrm{H}$ NMR (499.75 MHz, CDCl 3 ): $\delta 7.37-7.23$ (m, 15 H, 15×ArCH), 6.90 (t, $\left.J=1.8 \mathrm{~Hz}, 1 \mathrm{H}, \mathrm{H} 5\right), 4.66$ (td, $J=2.2,0.6 \mathrm{~Hz}, 1 \mathrm{H}, \mathrm{H} 4), 4.61$ (d, $J=11.9 \mathrm{~Hz}, 1 \mathrm{H}, \mathrm{CHHOBn}), 4.55-4.52$ (m, $\left.4 \mathrm{H}, 2 \times \mathrm{CH}_{2} \mathrm{OBn}\right), 4.50$ (d, $J=11.9 \mathrm{~Hz}, 1 \mathrm{H}, \mathrm{CH} H \mathrm{OBn}), 4.37$ (dd, $J=3.7,2.2 \mathrm{~Hz}, 1 \mathrm{H}, \mathrm{H} 3), 4.05$ (dd, $J=10.1,4.9 \mathrm{~Hz}, 1 \mathrm{H}, \mathrm{CHH}$ H2a), 4.03-3.99 (m, $1 \mathrm{H}, \mathrm{H} 2), 3.75$ (dd, $J=10.1,2.9 \mathrm{~Hz}, 1 \mathrm{H}, \mathrm{CH} H 2 \mathrm{a})$.

${ }^{13} \mathrm{C}$ NMR (125.67 MHz, $\left.\mathrm{CDCl}_{3}\right): \delta 137.7,137.2,137.1(3 \times \mathrm{ArC}), 133.2(\mathrm{C} 5), 128.7,128.6,128.5,128.24$, 128.21, 128.02, 127.99, 127.82, 127.77 (15× $\mathrm{ArCH}), 82.7$ (C4), 80.3 (C3), 77.5 (C2), 73.5, 71.9, 71.7

$\left(3 \times \mathrm{CH}_{2} \mathrm{OBn}\right), 66.0(\mathrm{C} 2 \mathrm{a})$.

LRMS (ESI) m/z: [M+ Na $]^{+} 440(100 \%)$.

$\underline{(2 R, 3 R, 4 R, 5 R)-3,4-B i s(\text { benzyloxy)-2-((benzyloxy)methyl)-5-vinylpyrrolidin-1-ol (S6) }}{ }^{9}$

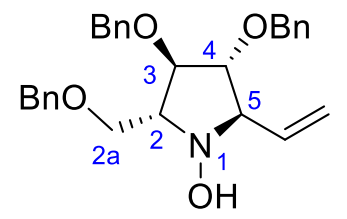

S6

To a solution of $\mathbf{S 5}(439 \mathrm{mg}, 1.05 \mathrm{mmol})$ in anhydrous THF $(1.10 \mathrm{~mL})$ at $0{ }^{\circ} \mathrm{C}$ (ice bath) under argon was added dropwise a $1.0 \mathrm{M}$ solution of vinylmagnesium bromide in THF (1.25 mL, $1.25 \mathrm{mmol})$. The reaction mixture was stirred at $0{ }^{\circ} \mathrm{C}$ for $30 \mathrm{~min}$ and quenched with sat. aq. $\mathrm{NaHCO}_{3}(10 \mathrm{~mL})$. The aqueous layer was extracted with EtOAc $(3 \times 10 \mathrm{~mL})$, and the combined organic layers were washed with sat. aq. $\mathrm{NaCl}(6$ $\mathrm{mL}$ ), dried over $\mathrm{Na}_{2} \mathrm{SO}_{4}$, filtered, and concentrated. The residue was purified by column chromatography (25:75 EtOAc/hexane), affording $\mathbf{S 6}$ (378 $\mathrm{mg}, 0.849 \mathrm{mmol})$ in $81 \%$ yield as a white solid. The analytical data were in agreement with the literature., ${ }^{9,13}$

$R_{\mathrm{f}}: 0.35$ (30:70 EtOAc/hexane).

$[\alpha]_{\mathrm{D}}^{25}-17.8\left(c 0.88, \mathrm{CHCl}_{3}\right)\left[\right.$ lit. $\left.^{13}[\alpha]_{\mathrm{D}}^{20}-19.6\left(c 0.66, \mathrm{CHCl}_{3}\right)\right]$. 
IR (neat) $\tilde{v}_{\max }\left(\mathrm{cm}^{-1}\right): 3329,3063,3026,2864,1496,1452,1362,1306,1207,1098,1027,995,921,864$, $729,692,661$.

${ }^{1} \mathrm{H}$ NMR (499.75 MHz, $\mathrm{CDCl}_{3}$ ): $\delta$ 7.35-7.23 (m, $\left.15 \mathrm{H}, 15 \times \mathrm{ArCH}\right), 6.04$ (ddd, $J=17.2,10.3,8.3 \mathrm{~Hz}, 1 \mathrm{H}$, $=\mathrm{CH}), 5.35-5.24\left(\mathrm{~m}, 2 \mathrm{H},=\mathrm{CH}_{2}\right), 4.58-4.41\left(\mathrm{~m}, 6 \mathrm{H}, 3 \times \mathrm{CH}_{2} \mathrm{OBn}\right), 3.98(\mathrm{t}, J=3.7 \mathrm{~Hz}, 1 \mathrm{H}, \mathrm{H} 3), 3.91(\mathrm{dd}$, $J=5.6,3.1 \mathrm{~Hz}, 1 \mathrm{H}, \mathrm{H} 4), 3.80-3.72$ (m, $2 \mathrm{H}, \mathrm{H} 5, \mathrm{CHH} \mathrm{H} 2 \mathrm{a}), 3.62$ (dd, $J=9.6,6.6 \mathrm{~Hz}, 1 \mathrm{H}, \mathrm{CH} H \mathrm{H} 2 \mathrm{a}$ ), 3.54-3.49 (m, $1 \mathrm{H}, \mathrm{H} 2)$.

${ }^{13} \mathrm{C}$ NMR (125.67 MHz, $\left.\mathrm{CDCl}_{3}\right): \delta 138.2,138.0,137.9(3 \times \mathrm{ArC}), 135.5(=\mathrm{CH}), 128.42,128.39,128.0$, 127.9, 127.8, 127.73, $127.68(15 \times \mathrm{ArCH}), 119.5\left(=\mathrm{CH}_{2}\right), 86.0(\mathrm{C} 4), 83.7(\mathrm{C} 3), 73.3\left(\mathrm{CH}_{2} \mathrm{OBn}\right), 72.8(\mathrm{C} 5)$, 71.9, $71.7\left(2 \times \mathrm{CH}_{2} \mathrm{OBn}\right), 69.6(\mathrm{C} 2), 67.7(\mathrm{C} 2 \mathrm{a})$.

LRMS (ESI) m/z: [M+ H] $446(100 \%)$.

$(2 R, 3 R, 4 R, 5 R)$-Benzyl 3,4-bis(benzyloxy)-2-((benzyloxy)methyl)-5-vinylpyrrolidine-1-carboxylate (9) $)^{9,13}$

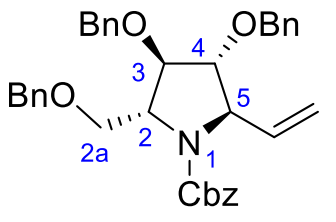

9

To a solution of $\mathbf{S 6}(378 \mathrm{mg}, 0.849 \mathrm{mmol})$ in glacial acetic acid $(4.0 \mathrm{~mL})$ was added water $(4.0 \mathrm{~mL})$ and activated zinc powder $(167 \mathrm{mg}, 2.55 \mathrm{mmol})$. The reaction mixture was stirred at $\mathrm{rt}$ for $8 \mathrm{~h}$, filtered through celite, and quenched by careful addition of solid $\mathrm{Na}_{2} \mathrm{CO}_{3}$ until no further gas evolution was observed. The mixture was extracted with $\mathrm{CH}_{2} \mathrm{Cl}_{2}(3 \times 10 \mathrm{~mL})$, and the combined organic layers were washed with sat. aq. $\mathrm{NaCl}(10 \mathrm{~mL})$, dried over $\mathrm{Na}_{2} \mathrm{SO}_{4}$, filtered, and concentrated. The residue was suspended in water (5.0 $\mathrm{mL}$ ), followed by the addition of solid $\mathrm{NaHCO}_{3}(178 \mathrm{mg}, 2.12 \mathrm{mmol})$. After the solid had completely dissolved, dioxane $(5.0 \mathrm{~mL})$ was added. The mixture was cooled to $0^{\circ} \mathrm{C}$ in an ice bath and stirred for $10 \mathrm{~min}$, followed by the addition of benzyl chloroformate $(145 \mu \mathrm{L}, 1.02 \mathrm{mmol})$. The reaction mixture was warmed to rt, stirred vigorously for $3 \mathrm{~h}$, and diluted with water $(6 \mathrm{~mL})$. The aqueous layer was extracted with EtOAc $(2 \times 10 \mathrm{~mL})$, and the combined organic layers were washed with $1 \mathrm{M}$ aq. $\mathrm{HCl}(5 \mathrm{~mL})$, sat. aq. $\mathrm{NaCl}(5 \mathrm{~mL})$, dried over $\mathrm{Na}_{2} \mathrm{SO}_{4}$, filtered, and concentrated. The residue was purified by column chromatography (10:90 EtOAc/hexane), affording 9 (431 mg, $0.765 \mathrm{mmol}$ ) in 90\% yield as a colorless oil. The analytical data were in agreement with the literature. ${ }^{9,14}$

$R_{\mathrm{f}}: 0.30$ (15:85 EtOAc/hexane).

$[\alpha]_{\mathrm{D}}^{25}-4.7\left(c 1.03, \mathrm{CHCl}_{3}\right)\left[\right.$ lit. $^{9}[\alpha]_{\mathrm{D}}^{24}-9.7\left(c 1.0, \mathrm{CHCl}_{3}\right)$, lit. $^{14}\left(\right.$ ent-9) $\left.[\alpha]_{\mathrm{D}}^{24}+5.4\left(c 0.74, \mathrm{CHCl}_{3}\right)\right]$. 
IR (neat) $\tilde{v}_{\max }\left(\mathrm{cm}^{-1}\right): 3030,2926,2863,1697,1496,1453,1403,1348,1211,1072,1028,986,914,850$, $770,733,695$.

${ }^{1} \mathrm{H}$ NMR (499.75 MHz, $\mathrm{CDCl}_{3}, 1.2: 1$ mixture of rotamers): $\delta 7.41-7.14(\mathrm{~m}, 20 \mathrm{H}, 20 \times \mathrm{ArCH}), 5.95-5.81$ (m, $1 \mathrm{H},=\mathrm{CH}), 5.29-5.00\left(\mathrm{~m}, 4 \mathrm{H}, \mathrm{CH}_{2} \mathrm{Cbz},=\mathrm{CH}_{2}\right), 4.67-4.28\left(\mathrm{~m}, 7.55 \mathrm{H}, 3 \times \mathrm{CH}_{2} \mathrm{OBn}, \mathrm{H} 5, \mathrm{H} 2\right), 4.23-$ 4.15 (m, $1.45 \mathrm{H}, \mathrm{H} 3, \mathrm{H} 2 *), 4.02$ (dd, $J=8.7,4.3 \mathrm{~Hz}, 0.55 \mathrm{H}, \mathrm{CHH} \mathrm{H} 2 \mathrm{a}), 3.85$ (d, J=5.7 Hz, $1 \mathrm{H}, \mathrm{H} 4)$, 3.73 (dd, $\left.J=9.0,4.2 \mathrm{~Hz}, 0.45 \mathrm{H}, \mathrm{CHH} \mathrm{H} 2 \mathrm{a}^{*}\right), 3.57-3.48$ (m, $\left.1 \mathrm{H}, \mathrm{CH} H \mathrm{H} 2 \mathrm{a}\right)$.

${ }^{13} \mathrm{C}$ NMR (125.67 MHz, $\mathrm{CDCl}_{3}, 1.2: 1$ mixture of rotamers): $\delta 155.1,154.4^{*}(\mathrm{C}=\mathrm{O} \mathrm{Cbz}), 138.6,138.3$, 137.9, 137.8, 137.5, $136.6(4 \times \mathrm{ArC} \mathrm{OBn} / \mathrm{Cbz}), 136.8,136.3 *(=\mathrm{CH}), 128.59,128.56,128.50,128.46$, $128.43,128.39,128.3,128.13,128.06,127.93,127.90,127.79,127.76,127.74,127.70,127.6(20 \times \mathrm{ArCH}$ $\mathrm{OBn} / \mathrm{Cbz}) .116 .9 *, 116.5\left(=\mathrm{CH}_{2}\right), 86.9,86.0^{*}(\mathrm{C} 4), 82.9^{*}, 81.6(\mathrm{C} 3), 73.14,73.08^{*}, 71.46^{*}, 71.44,71.2^{*}$, $71.0\left(3 \times \mathrm{CH}_{2} \mathrm{OBn}\right), 68.8 *, 68.0(\mathrm{C} 2 \mathrm{a}), 67.23 *, 67.19(\mathrm{C} 5), 67.0\left(\mathrm{CH}_{2} \mathrm{Cbz}\right), 63.2,62.9 *(\mathrm{C} 2)$.

LRMS (ESI) m/z: [M+ Na $]^{+} 586(100 \%)$.

$(2 R, 3 R, 4 R, 5 R)$-Benzyl 3,4-bis(benzyloxy)-2-((benzyloxy)methyl)-5-((S)-1,2-epoxyethyl)pyrrolidine-1carboxylate (10a) and $(2 R, 3 R, 4 R, 5 R)$-Benzyl 3,4-bis(benzyloxy)-2-((benzyloxy)methyl)-5- $((R)-1,2$-epoxyethyl)pyrrolidine-1-carboxylate $(\mathbf{1 0 b})$

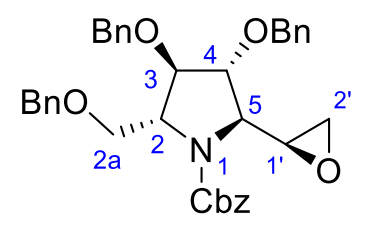

$10 \mathrm{a}$

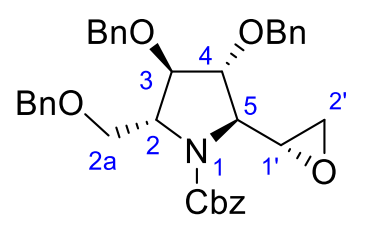

$10 \mathrm{~b}$

To a solution of $9(431 \mathrm{mg}, 0.765 \mathrm{mmol})$ in $\mathrm{CH}_{2} \mathrm{Cl}_{2}(7.7 \mathrm{~mL})$ was added $\mathrm{Na}_{2} \mathrm{HPO}_{4} \cdot 2 \mathrm{H}_{2} \mathrm{O}(545 \mathrm{mg}, 3.06$ mmol) and $m$-CPBA (1.06 g, $6.12 \mathrm{mmol}, 99 \%) .{ }^{15}$ The reaction mixture was stirred at $\mathrm{rt}$ for $19 \mathrm{~h}$, diluted with $\mathrm{Et}_{2} \mathrm{O}(95 \mathrm{~mL})$, and washed with $10 \%(\mathrm{w} / \mathrm{v})$ aq. $\mathrm{NaOH}(2 \times 40 \mathrm{~mL})$. The combined aqueous layers were back-extracted with $\mathrm{Et}_{2} \mathrm{O}(4 \times 25 \mathrm{~mL})$, and the combined organic layers were washed with sat. aq. $\mathrm{NaCl}$ $(50 \mathrm{~mL})$, dried over $\mathrm{Na}_{2} \mathrm{SO}_{4}$, filtered, and concentrated. The residue was purified by column chromatography (10:90 $\rightarrow 20: 80 \mathrm{EtOAc} /$ hexane), affording 10a (254 mg, $0.439 \mathrm{mmol})$ and $\mathbf{1 0 b}$ (127 mg, $0.219 \mathrm{mmol})$ in $57 \%$ and $29 \%$ yields, respectively, as colorless oils.

\section{$\underline{10 a}$}

$R_{\mathrm{f}}: 0.35$ (20:80 EtOAc/hexane).

$[\alpha]_{\mathrm{D}}^{25}-25.1\left(c 1.03, \mathrm{CHCl}_{3}\right)$. 
IR (neat) $\tilde{v}_{\max }\left(\mathrm{cm}^{-1}\right): 3062,3031,2926,2864,1700,1496,1453,1403,1346,1256,1206,1087,1027,966$, 920, 857, 807, 769, 734, 695, 641 .

${ }^{1} \mathrm{H}$ NMR (400.16 MHz, $\mathrm{CDCl}_{3}, 1: 1$ mixture of rotamers): $\delta 7.43-7.10(\mathrm{~m}, 20 \mathrm{H}, 20 \times \mathrm{ArCH}), 5.19,5.18$ $(2 \times \mathrm{d}, J=12.4,11.8 \mathrm{~Hz}, 1 \mathrm{H}, \mathrm{CHHCbz}), 5.03$ (d, $J=12.4 \mathrm{~Hz}, 0.5 \mathrm{H}, \mathrm{CH} H \mathrm{Cbz}), 4.97$ (d, $J=11.8 \mathrm{~Hz}, 0.5$ $\mathrm{H}, \mathrm{CH} H \mathrm{Cbz}$ ), 4.66-4.29 (m, $\left.6.5 \mathrm{H}, 3 \times \mathrm{CH}_{2} \mathrm{OBn}, \mathrm{H} 2\right), 4.26-4.18$ (m, $\left.2.5 \mathrm{H}, \mathrm{H} 2, \mathrm{H} 3, \mathrm{H} 4\right), 4.01$ (dd, J= 8.8, $4.4 \mathrm{~Hz}, 0.5 \mathrm{H}, \mathrm{CHH}$ H2a), 3.72 (dd, $J=9.0,4.2 \mathrm{~Hz}, 1 \mathrm{H}, \mathrm{CHH} \mathrm{H} 2 \mathrm{a}), 3.57-3.49$ (m, $1 \mathrm{H}, \mathrm{CH} H \mathrm{H} 2 \mathrm{a}), 3.47$ (d, $J=8.7 \mathrm{~Hz}, 0.5 \mathrm{H}, \mathrm{H} 5), 3.35$ (d, $J=8.7 \mathrm{~Hz}, 0.5 \mathrm{H}, \mathrm{H} 5), 3.09$ (dt, $J=8.7,3.1 \mathrm{~Hz}, 0.5 \mathrm{H}, \mathrm{H1}$ '), 3.02 (dt, $J$ = 8.8, 3.1 Hz, 0.5 H, H1'), 2.99-2.94 (m, 1 H, $\left.\mathrm{CH}_{2} \mathrm{H} 2^{\prime}\right), 2.56$ (dd, $\left.J=5.1,3.7 \mathrm{~Hz}, 0.5 \mathrm{H}, \mathrm{CHH} \mathrm{H} 2^{\prime}\right), 2.41$ (dd, $J=5.1,2.5 \mathrm{~Hz}, 0.5 \mathrm{H}, \mathrm{CH} H \mathrm{H} 2^{\prime}$ ).

${ }^{13} \mathrm{C}$ NMR (100.63 MHz, $\mathrm{CDCl}_{3}, 1: 1$ mixture of rotamers): $\delta 154.91,154.84(\mathrm{C}=\mathrm{O}), 138.5,138.3,137.7$, 137.6, 137.53, 137.49 (3×ArC OBn), 136.4, 136.1 (ArC Cbz), 128.8, 128.7, 128.68, 128.6, 128.56, 128.54, 128.5, 128.3, 128.2, 127.93, 127.91, 127.9, 127.8, 127.7, 127.6 (20×ArCH OBn/Cbz), 84.8, 83.6 (C4), 82.5, 81.1 (C3), 73.22, $73.16\left(\mathrm{CH}_{2} \mathrm{OBn}(\mathrm{C} 2 \mathrm{a})\right), 71.6,71.5,71.3,71.2\left(2 \times \mathrm{CH}_{2} \mathrm{OBn}\right), 68.6,67.7$ (C2a), 67.6, 67.3 $\left(\mathrm{CH}_{2} \mathrm{Cbz}\right), 67.0,66.5(\mathrm{C} 5), 63.9,63.6(\mathrm{C} 2), 51.6,51.3\left(\mathrm{C} 1^{\prime}\right), 51.0,50.7\left(\mathrm{C}^{\prime}\right)$.

HRMS (ESI) m/z: [M + Na $]^{+}$Calcd for $\mathrm{C}_{36} \mathrm{H}_{37} \mathrm{NO}_{6} \mathrm{Na}$ 602.2519; Found 602.2547.

\section{$\underline{10 b}$}

$R_{\mathrm{f}}: 0.20$ (20:80 EtOAc/hexane).

$[\alpha]_{\mathrm{D}}^{25}-29.0\left(c 0.46, \mathrm{CHCl}_{3}\right)$.

IR (neat) $\tilde{v}_{\max }\left(\mathrm{cm}^{-1}\right): 3031,2924,2863,1698,1496,1453,1403,1348,1259,1205,1087,1027,912,873$, $815,770,734,695,641$.

${ }^{1} \mathrm{H}$ NMR (400.16 MHz, $\mathrm{CDCl}_{3}, 1.2: 1$ mixture of rotamers): $\delta 7.49-7.11(\mathrm{~m}, 20 \mathrm{H}, 20 \times \mathrm{ArCH}), 5.24-5.04$ (m, $2 \mathrm{H}, \mathrm{CH}_{2} \mathrm{Cbz}$ ), 4.66-4.30 (m, 6.55 H, 3× $\mathrm{CH}_{2} \mathrm{OBn}, \mathrm{H} 2$ ), 4.20 (dd, $J=10.6,4.0 \mathrm{~Hz}, 0.45 \mathrm{H}, \mathrm{H} 2 *$ ), 4.174.11 (m, 1 H, H3), 4.01 (dd, $J=8.9,4.2 \mathrm{~Hz}, 0.55 \mathrm{H}, \mathrm{CHH} \mathrm{H} 2 \mathrm{a}), 3.93$ (d, J=6.0 Hz, $0.45 \mathrm{H}, \mathrm{H} 5 *$ ), 3.85 (br, $1 \mathrm{H}, \mathrm{H} 4), 3.77$ (d, $J=6.1 \mathrm{~Hz}, 0.55 \mathrm{H}, \mathrm{H} 5), 3.71$ (dd, $J=9.1,4.1 \mathrm{~Hz}, 0.45 \mathrm{H}, \mathrm{CHH} \mathrm{H} 2 \mathrm{a} *), 3.57-3.45$ (m, 1 $\mathrm{H}, \mathrm{CH} H \mathrm{H} 2 \mathrm{a}$ ), 3.36 (br, $\left.0.45 \mathrm{H}, \mathrm{H} 1^{\prime *}\right), 3.19$ (br, $\left.0.55 \mathrm{H}, \mathrm{H} 1^{\prime}\right), 2.68-2.54$ (m, $1.45 \mathrm{H}, \mathrm{CH}_{2} \mathrm{H} 2^{\prime}, \mathrm{CHH} \mathrm{H} 2^{\prime *}$ ), 2.52-2.47 (m, 0.55 H, CHH H2').

${ }^{13} \mathrm{C} \mathrm{NMR}\left(100.63 \mathrm{MHz}, \mathrm{CDCl}_{3}, 1.2: 1\right.$ mixture of rotamers): $\delta 155.2,154.8^{*}(\mathrm{C}=\mathrm{O}), 138.5,138.3^{*}, 137.7$, 137.4 (3× $\mathrm{ArC} \mathrm{OBn}), 136.6,136.5^{*}$ (ArC Cbz), 128.6, 128.56, 128.5, 128.2, 128.0, 127.9, 127.86, 127.8, $127.7(20 \times \mathrm{ArCH} \mathrm{OBn} / \mathrm{Cbz}), 84.7,83.1^{*}(\mathrm{C} 4), 82.3^{*}, 80.9$ (C3), 73.2, 71.5*, 71.4, 71.3*, 71.2 
$\left(3 \times \mathrm{CH}_{2} \mathrm{OBn}\right), 68.5^{*}, 67.7(\mathrm{C} 2 \mathrm{a}), 67.4\left(\mathrm{CH}_{2} \mathrm{Cbz}\right), 66.3,66.2 *(\mathrm{C} 5), 63.6,63.4 *(\mathrm{C} 2), 53.2,52.3 *\left(\mathrm{C} 1^{\prime}\right), 44.3$, $44.2^{*}\left(\mathrm{C} 2^{\prime}\right)$.

HRMS (ESI) m/z: [M + Na $]^{+}$Calcd for $\mathrm{C}_{36} \mathrm{H}_{37} \mathrm{NO}_{6} \mathrm{Na}$ 602.2519; Found 602.2509.

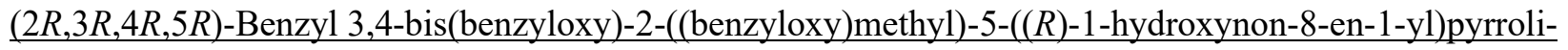
dine-1-carboxylate (6a)

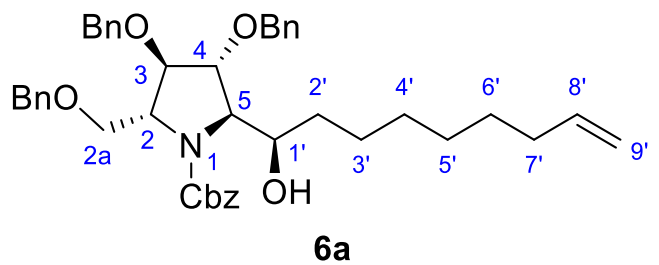

To an unstirred suspension of magnesium turnings $(112 \mathrm{mg}, 4.60 \mathrm{mmol})$ and a crystal of iodine in anhydrous THF $(0.80 \mathrm{~mL})$ at $45^{\circ} \mathrm{C}$ under argon was added a few drops of a solution of 7-bromo-1-heptene (900 $\mathrm{mg}$, $5.06 \mathrm{mmol})$ in anhydrous THF $(5.0 \mathrm{~mL})$. Once initiation was observed, the reaction mixture was stirred, and the remaining volume of bromide solution was added dropwise over 30 min using a syringe pump. Once the addition was complete, the reaction mixture was heated at a gentle reflux, stirred for $2 \mathrm{~h}$, and cooled to rt, affording a dark grey solution. An aliquot of the solution was titrated against a solution of iodine $/ \mathrm{LiCl}$ in THF, and the concentration of the Grignard reagent was determined to be $0.453 \mathrm{M}$. In a separate flask, CuBr·DMS (234 mg, $1.15 \mathrm{mmol}$ ) was weighed out, sealed, flushed with argon, and suspended in anhydrous THF $(1.0 \mathrm{~mL})$. The suspension was cooled to $-40{ }^{\circ} \mathrm{C}$ using an immersion-cooled cold bath and stirred, followed by the dropwise addition of the Grignard reagent $(5.10 \mathrm{~mL}, 2.31 \mathrm{mmol})$. The reaction mixture was stirred at $-40{ }^{\circ} \mathrm{C}$ for $1 \mathrm{~h}$, during which time the initial white color of the suspension changed to yellow, orange, and finally dark purple. A solution of $\mathbf{1 0 a}(133 \mathrm{mg}, 0.230 \mathrm{mmol})$ in anhydrous THF $(0.80 \mathrm{~mL})$ was prepared and added dropwise to the reaction vessel, and the flask was rinsed in with additional THF $(0.20 \mathrm{~mL})$. The reaction mixture was stirred at $-40{ }^{\circ} \mathrm{C}$ for $24 \mathrm{~h}$, quenched carefully with sat. aq. $\mathrm{NH}_{4} \mathrm{Cl}(2 \mathrm{~mL})$, and stirred vigorously for $10 \mathrm{~min}$, affording a royal blue organic layer. The mixture was warmed to $\mathrm{rt}$, and the aqueous layer was extracted with EtOAc $(4 \times 5 \mathrm{~mL})$. The combined organic layers were washed with sat. aq. $\mathrm{NaCl}(10 \mathrm{~mL})$, dried over $\mathrm{Na}_{2} \mathrm{SO}_{4}$, filtered, and concentrated. The residue was purified by column chromatography $(0: 100 \rightarrow 20: 80$ EtOAc/hexane) and concentrated in vacuo at 50 ${ }^{\circ} \mathrm{C}$ for $2 \mathrm{~h}$ to remove remaining traces of hept-6-en-1-ol, affording $6 \mathbf{6 a}(131 \mathrm{mg}, 0.193 \mathrm{mmol})$ in 84\% yield as a colorless oil.

$R_{\mathrm{f}}: 0.30$ (20:80 EtOAc/hexane).

$[\alpha]_{\mathrm{D}}^{25}-31.6\left(c 1.42, \mathrm{CHCl}_{3}\right)$. 
IR (neat) $\tilde{v}_{\max }\left(\mathrm{cm}^{-1}\right): 3459,3031,2925,2855,1699,1496,1453,1405,1345,1207,1073,1028,909,734$, $696,605$.

${ }^{1} \mathrm{H}$ NMR (400.16 MHz, $\mathrm{CDCl}_{3}, 1.6: 1$ mixture of rotamers): $\delta 7.38-7.13(\mathrm{~m}, 20 \mathrm{H}, 20 \times \mathrm{ArCH}), 5.80$ (ddt, $J$ $=16.9,10.2,6.6 \mathrm{~Hz}, 1 \mathrm{H},=\mathrm{CH}), 5.21-5.14(\mathrm{~m}, 1 \mathrm{H}, \mathrm{CHHCbz}), 5.06,5.05(2 \times \mathrm{d}, J=12.4,12.3 \mathrm{~Hz}, 1 \mathrm{H}$, $\mathrm{CH} H \mathrm{Cbz}), 5.02-4.89\left(\mathrm{~m}, 2 \mathrm{H},=\mathrm{CH}_{2}\right), 4.67-4.12\left(\mathrm{~m}, 8.62 \mathrm{H}, 3 \times \mathrm{CH}_{2} \mathrm{OBn}, \mathrm{H} 2, \mathrm{H} 3, \mathrm{H} 1^{\prime}\right), 4.10-3.98$ (m, 2.38 H, H4, H5, CHH H2a*), 3.90 (br, 0.38 H, H1'*), 3.69 (dd, J=8.9, 4.3 Hz, 0.62 H, CHH H2a), 3.52-3.42 (m, 1.62 H, CHH H2a, OH), 3.30 (d, $\left.J=6.0 \mathrm{~Hz}, 0.38 \mathrm{H}, \mathrm{OH}^{*}\right), 2.07-1.97$ (m, $2 \mathrm{H}, \mathrm{CH}_{2} \mathrm{H} 7$ '), 1.59-1.06 (m, $\left.10 \mathrm{H}, 5 \times \mathrm{CH}_{2} \mathrm{H} 2^{\prime}, \mathrm{H} 3^{\prime}, \mathrm{H} 4^{\prime}, \mathrm{H} 5^{\prime}, \mathrm{H}^{\prime}\right)$.

${ }^{13} \mathrm{C} \mathrm{NMR}\left(100.63 \mathrm{MHz}, \mathrm{CDCl}_{3}, 1.6: 1\right.$ mixture of rotamers): $\delta 154.9,154.7(\mathrm{C}=\mathrm{O} \mathrm{Cbz}), 139.3,139.2(=\mathrm{CH})$, $138.5^{*}, 138.2,137.4,137.3^{*}, 136.8,136.6^{*}, 136.4,136.2 *$ (4×ArC OBn/Cbz), 128.7, 128.63, 128.59, 128.5, 128.3, 128.23, 128.2, 128.1, 127.8, 127.76, 127.7 (20× $\mathrm{ArCH} \mathrm{OBn} / \mathrm{Cbz}), 114.3,114.2\left(=\mathrm{CH}_{2}\right), 81.8$ (C4), 81.4, 80.0*(C3), 73.2, 71.4, 71.3 (6× $\left.\mathrm{CH}_{2} \mathrm{OBn}\right), 70.4 *$, 70.2 (C5), 69.6, 69.4*(C1'), $68.4(\mathrm{C} 2 \mathrm{a}), 67.38$, 67.35, 67.3 (C2a*, $\left.\mathrm{CH}_{2} \mathrm{Cbz}\right), 63.4^{*}, 63.2$ (C2), 35.7*, 34.6, 29.6, 29.4*, 29.2, 29.1*, 29.0, 28.9*, 26.3, $26.1^{*}\left(\mathrm{C} 2^{\prime}, \mathrm{C}^{\prime}, \mathrm{C}^{\prime}, \mathrm{C} 5^{\prime}, \mathrm{C} 6^{\prime}\right), 33.9\left(\mathrm{C}^{\prime}\right)$.

HRMS (ESI) m/z: [M + Na $]^{+}$Calcd for $\mathrm{C}_{43} \mathrm{H}_{51} \mathrm{NO}_{6} \mathrm{Na}$ 700.3614; Found 700.3624.

$(1 R, 5 R, 6 R, 7 R, 7 \mathrm{a} R)-1-($ Oct-7-en-1-yl)-6,7-bis(benzyloxy)-5-((benzyloxy)methyl)tetrahydropyrrolo[1,2c]oxazol-3(1H)-one (11a)

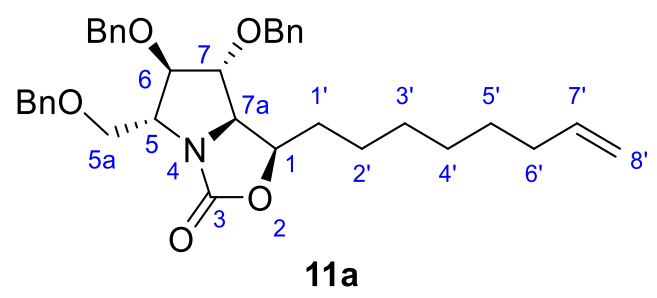

To a solution of $\mathbf{6 a}(28.0 \mathrm{mg}, 0.0413 \mathrm{mmol})$ in anhydrous THF $(1.4 \mathrm{~mL})$ at $0{ }^{\circ} \mathrm{C}$ (ice bath) was added sodium hydride $(60 \% \mathrm{w} / \mathrm{w}$ in mineral oil, $10.7 \mathrm{mg}, 0.267 \mathrm{mmol})$. The reaction mixture was warmed to $\mathrm{rt}$, stirred for $3 \mathrm{~h}$, cooled to $0{ }^{\circ} \mathrm{C}$, and carefully quenched with sat. aq. $\mathrm{NH}_{4} \mathrm{Cl}(1.5 \mathrm{~mL})$. The aqueous layer was extracted with EtOAc $(3 \times 1.5 \mathrm{~mL})$, and the combined organic layers were dried over $\mathrm{Na}_{2} \mathrm{SO}_{4}$, filtered, and concentrated. The residue was purified by column chromatography $(0: 100 \rightarrow 20: 80$ EtOAc/hexane), affording 11 a (23.3 mg, $0.0409 \mathrm{mmol}$ ) in 99\% yield as a pale-yellow oil.

$R_{\mathrm{f}}: 0.30$ (20:80 EtOAc/hexane).

$[\alpha]_{\mathrm{D}}^{25}+7.0\left(c 1.17, \mathrm{CHCl}_{3}\right)$. 
IR (neat) $\tilde{v}_{\max }\left(\mathrm{cm}^{-1}\right): 3031,2925,2855,1755,1640,1497,1454,1360,1304,1207,1065,1028,909,821$, $779,735,696,609$.

${ }^{1} \mathrm{H}$ NMR (400.16 MHz, $\mathrm{CDCl}_{3}$ ): $\delta$ 7.39-7.18 (m, $\left.15 \mathrm{H}, 15 \times \mathrm{ArCH}\right), 5.79$ (ddt, $J=16.9,10.2,6.7 \mathrm{~Hz}, 1 \mathrm{H}$, $=\mathrm{CH}), 5.03-4.89\left(\mathrm{~m}, 2 \mathrm{H},=\mathrm{CH}_{2}\right), 4.64-4.50\left(\mathrm{~m}, 5 \mathrm{H}, \mathrm{CH}_{2} \mathrm{OBn}, 2 \times \mathrm{CHHOBn}, \mathrm{H} 1\right), 4.47(\mathrm{~d}, J=11.6 \mathrm{~Hz}, 1$ H, CHHOBn), 4.42 (d, $J=11.6 \mathrm{~Hz}, 1 \mathrm{H}, \mathrm{CH} H \mathrm{OBn}), 4.22$ (dd, $J=4.3,3.1 \mathrm{~Hz}, 1 \mathrm{H}, \mathrm{H} 6), 4.15$ (td, $J=5.6$, $3.1 \mathrm{~Hz}, 1 \mathrm{H}, \mathrm{H} 5), 4.01$ (dd, $J=7.8,4.3 \mathrm{~Hz}, 1 \mathrm{H}, \mathrm{H} 7$ ), 3.89 (t, $J=7.8 \mathrm{~Hz}, 1 \mathrm{H}, \mathrm{H} 7 \mathrm{a}), 3.60-3.51$ (m, $2 \mathrm{H}$, $\mathrm{CH}_{2} \mathrm{H} 5 \mathrm{a}$ ), 2.07-1.97 (m, 2 H, $\mathrm{CH}_{2} \mathrm{H6}^{\prime}$ ), 1.66-1.17 (m, 10 H, 5× $\mathrm{CH}_{2} \mathrm{H}^{\prime}, \mathrm{H}^{\prime}$, H3', H4', H5').

${ }^{13} \mathrm{C}$ NMR (100.63 MHz, $\left.\mathrm{CDCl}_{3}\right): \delta 160.8(\mathrm{C}=\mathrm{O}), 139.1(=\mathrm{CH}), 137.9,137.6,137.3(3 \times \mathrm{ArC}), 128.6,128.57$, 128.3, 128.2, 128.1, 127.9, $127.8(15 \times \mathrm{ArCH}), 114.4\left(=\mathrm{CH}_{2}\right), 86.7$ (C6), 82.5 (C7), 76.8 (C1), 73.5, 72.3, $72.2\left(3 \times \mathrm{CH}_{2} \mathrm{OBn}\right), 70.5$ (C5a), 64.8 (C7a), $62.6(\mathrm{C} 5), 33.8$ (C6'), $30.4\left(\mathrm{Cl}^{\prime}\right), 29.3,29.0,28.9,26.0$ (C2', $\left.\mathrm{C} 3^{\prime}, \mathrm{C} 4^{\prime}, \mathrm{C} 5^{\prime}\right)$.

HRMS (ESI) m/z: [M + Na] $]^{+}$Calcd for $\mathrm{C}_{36} \mathrm{H}_{43} \mathrm{NO}_{5} \mathrm{Na}$ 592.3039; Found 592.3059 .

(2R,3R,4R,5R)-Benzyl 3,4-bis(benzyloxy)-2-((benzyloxy)methyl)-5-((S)-1-hydroxynon-8-en-1-yl)pyrrolidine-1-carboxylate $(\mathbf{6 b})$

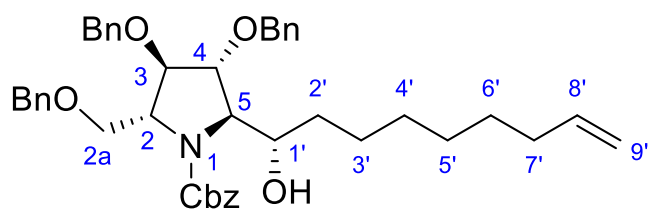

$6 b$

The reaction was carried out as above for 10a starting from $10 \mathrm{~b}(52.6 \mathrm{mg}, 0.0907 \mathrm{mmol})$, affording an inseparable mixture of $\mathbf{6 b}(28.2 \mathrm{mg}, 0.0416 \mathrm{mmol})$ and $\mathbf{1 1 b}(17.2 \mathrm{mg}, 0.0302 \mathrm{mmol})$ in $46 \%$ and $33 \%$ yields, respectively, as a yellow oil. ${ }^{16}$

$R_{\mathrm{f}}: 0.45$ (25:75 EtOAc/hexane).

IR (neat) $\tilde{v}_{\max }\left(\mathrm{cm}^{-1}\right): 3454,3031,2925,2855,1758,1698,1496,1453,1407,1349,1209,1091,1070,1027$, 994, 909, 733, 696.

${ }^{1} \mathrm{H}$ NMR (400.16 MHz, $\mathrm{CDCl}_{3}$, 6b only): $\delta$ 7.40-7.13 (m, 20 H, 20×ArCH OBn/Cbz), 5.87-5.72 (m, $1 \mathrm{H}$, $=\mathrm{CH}), 5.16(\mathrm{~d}, J=12.2 \mathrm{~Hz}, 1 \mathrm{H}, \mathrm{CHHCbz}), 5.09(\mathrm{~d}, J=12.2 \mathrm{~Hz}, 1 \mathrm{H}, \mathrm{CH} H \mathrm{Cbz}), 5.03-4.89\left(\mathrm{~m}, 2 \mathrm{H},=\mathrm{CH}_{2}\right)$, 4.65-4.30 (m, $\left.6 \mathrm{H}, 3 \times \mathrm{CH}_{2} \mathrm{OBn}\right), 4.21-4.13$ (m, $\left.2 \mathrm{H}, \mathrm{H} 2, \mathrm{H} 3\right), 4.04$ (d, J= $\left.7.9 \mathrm{~Hz}, 1 \mathrm{H}, \mathrm{H} 5\right), 3.90$ (br, $1 \mathrm{H}$, H1'), 3.84 (br, $1 \mathrm{H}, \mathrm{H} 4), 3.81$ (dd, $J=8.9,4.1 \mathrm{~Hz}, 1 \mathrm{H}, \mathrm{CHH} \mathrm{H} 2 \mathrm{a}), 3.48$ (dd, $J=10.5,8.9 \mathrm{~Hz}, 1 \mathrm{H}, \mathrm{CH} H$ H2a), 2.09-1.96 (m, 2 H, CH $\left.2 \mathrm{H}^{\prime}\right), 1.75-1.06$ (m, 10 H, 5× $\left.\mathrm{CH}_{2} \mathrm{H1}^{\prime}, \mathrm{H} 2^{\prime}, \mathrm{H} 3^{\prime}, \mathrm{H} 4^{\prime}, \mathrm{H} 5^{\prime}\right)$. 
${ }^{13} \mathrm{C}$ NMR (100.63 MHz, $\mathrm{CDCl}_{3}$, 6b only): $\delta 157.4(\mathrm{C}=\mathrm{O}), 139.3(=\mathrm{CH}), 138.3,137.6,137.5,136.2(4 \times \mathrm{ArC}$ $\mathrm{OBn} / \mathrm{Cbz}), 128.70,128.68,128.6,128.53,128.47,128.3,128.1,128.0,127.9,127.8,127.7(20 \times \mathrm{ArCH}$ $\mathrm{OBn} / \mathrm{Cbz}), 114.3\left(=\mathrm{CH}_{2}\right), 83.1(\mathrm{C} 4), 82.2(\mathrm{C} 3), 73.5\left(\mathrm{C} 1^{\prime}\right), 73.1,71.3,71.1\left(3 \times \mathrm{CH}_{2} \mathrm{OBn}\right), 70.1(\mathrm{C} 5), 68.5$ (C2a), 67.8 ( $\left.\mathrm{CH}_{2} \mathrm{Cbz}\right), 63.3(\mathrm{C} 2), 35.4(\mathrm{C} 2$ '), 33.9 (C7'), 29.6, 29.2, 29.0, 25.3 (C3', C4', C5', C6').

HRMS (ESI) m/z: [M + Na] $]^{+}$Calcd for $\mathrm{C}_{43} \mathrm{H}_{51} \mathrm{NO}_{6} \mathrm{Na}$ 700.3614; Found 700.3616.

$(1 S, 5 R, 6 R, 7 R, 7 \mathrm{a} R)-1-($ Oct-7-en-1-yl)-6,7-bis(benzyloxy)-5-((benzyloxy)methyl)tetrahydropyrrolo[1,2c]oxazol-3(1H)-one (11b)

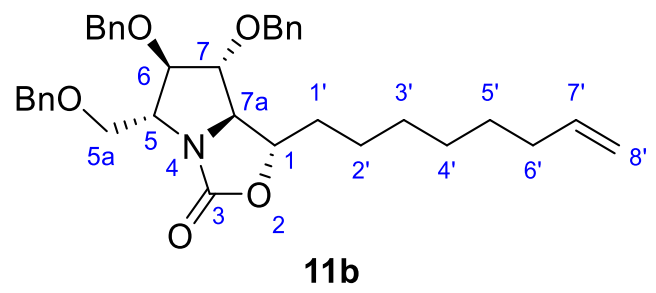

The reaction was carried out as above for $\mathbf{6 a}$ starting from a mixture of $\mathbf{6 b}(12.8 \mathrm{mg}, 0.0189 \mathrm{mmol})$ and $\mathbf{1 1 b}$ (7.8 mg, $0.0137 \mathrm{mmol})$, affording $11 \mathbf{b}(17.0 \mathrm{mg}, 0.0298 \mathrm{mmol})$ in $92 \%$ yield as a pale-yellow oil.

$R_{\mathrm{f}}: 0.45$ (25:75 EtOAc/hexane).

$[\alpha]_{\mathrm{D}}^{25}-27.8\left(c 0.85, \mathrm{CHCl}_{3}\right)$

IR (neat) $\tilde{v}_{\max }\left(\mathrm{cm}^{-1}\right): 3031,2925,2855,1755,1496,1454,1363,1214,1096,1060,1027,909,734,696$.

${ }^{1} \mathrm{H}$ NMR (400.16 MHz, $\left.\mathrm{CDCl}_{3}\right): \delta 7.38-7.20(\mathrm{~m}, 15 \mathrm{H}, 15 \times \mathrm{ArCH}), 5.80(\mathrm{ddt}, J=17.0,10.2,6.7 \mathrm{~Hz}, 1 \mathrm{H}$, $=\mathrm{CH}), 4.99(\mathrm{dq}, J=17.0,1.7 \mathrm{~Hz}, 1 \mathrm{H},=\mathrm{CHH}), 4.94(\mathrm{ddt}, J=10.2,2.3,1.2 \mathrm{~Hz}, 1 \mathrm{H},=\mathrm{CH} H), 4.60(\mathrm{~d}, 11.8$ $\mathrm{Hz}, 1 \mathrm{H}, \mathrm{C} H \mathrm{HOBn}), 4.59(\mathrm{~d}, J=12.0 \mathrm{~Hz}, 1 \mathrm{H}, \mathrm{CH} H \mathrm{OBn}), 4.54\left(\mathrm{ABq}, \Delta \delta_{\mathrm{AB}}=0.01, J_{\mathrm{AB}}=12.1 \mathrm{~Hz}, 2 \mathrm{H}\right.$, $\mathrm{CH}_{2} \mathrm{OBn}$ ), 4.46 (d, $\left.J=11.8 \mathrm{~Hz}, 1 \mathrm{H}, \mathrm{CH} H \mathrm{OBn}\right), 4.45$ (d, $\left.J=12.0 \mathrm{~Hz}, 1 \mathrm{H}, \mathrm{CH} H \mathrm{OBn}\right), 4.26-4.20$ (m, $2 \mathrm{H}$, H1, H6), 4.16-4.10 (m, 1 H, H5), 3.86 (dd, $J=6.3,4.0$ Hz, 1 H, H7), 3.59-3.50 (m, 3 H, CH $\mathrm{CH}_{2}$, H7a), 2.08-2.00 (m, 2 H, $\left.\mathrm{CH}_{2} \mathrm{H} 6^{\prime}\right), 1.76-1.49$ (m, $2 \mathrm{H}, \mathrm{CH}_{2} \mathrm{H}^{\prime}$ ), 1.46-1.19 (m, $8 \mathrm{H}, 4 \times \mathrm{CH}_{2} \mathrm{H} 2^{\prime}, \mathrm{H}^{\prime}, \mathrm{H}^{\prime}, \mathrm{H}^{\prime}$ ).

${ }^{13} \mathrm{C} \mathrm{NMR}\left(100.63 \mathrm{MHz}, \mathrm{CDCl}_{3}\right): \delta 160.7(\mathrm{C}=\mathrm{O}), 139.1(=\mathrm{CH}), 138.0,137.6,137.5(3 \times \mathrm{ArC}), 128.7,128.59$, 128.55, 128.3, 128.1, 128.0, 127.90, 127.87, $127.8(15 \times \mathrm{ArCH}), 114.5\left(=\mathrm{CH}_{2}\right), 88.1$ (C7), $85.8(\mathrm{C} 6), 80.3$ (C1), 73.4, 72.6, 72.1 (3× $\left.\mathrm{CH}_{2} \mathrm{OBn}\right), 69.8$ (C5a), 67.5 (C7a), 62.4 (C5), 35.4 (C1'), 33.8 (C6'), 29.2, 29.0, 28.9, 24.6 (C2', C3', C4', C5').

HRMS (ESI) m/z: [M + Na] $]^{+}$Calcd for $\mathrm{C}_{36} \mathrm{H}_{43} \mathrm{NO}_{5} \mathrm{Na}$ 592.3039; Found 592.3044. 
$\underline{4-A l l y l p h e n o l ~(S 7)})^{17}$

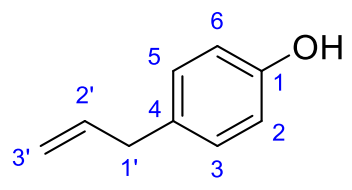

S8

To a solution of 4-allylanisole $(\mathbf{1 2}, 481 \mathrm{mg}, 3.25 \mathrm{mmol})$ in anhydrous $\mathrm{CH}_{2} \mathrm{Cl}_{2}(11 \mathrm{~mL})$ at $0{ }^{\circ} \mathrm{C}$ (ice bath) was added dropwise a $1.0 \mathrm{M}$ solution of $\mathrm{BBr}_{3}(3.60 \mathrm{~mL}, 3.60 \mathrm{mmol})$ in $\mathrm{CH}_{2} \mathrm{Cl}_{2}$. The reaction mixture was stirred at $0{ }^{\circ} \mathrm{C}$ for $1 \mathrm{~h}$, carefully quenched with water $(7.5 \mathrm{~mL})$, warmed to $\mathrm{rt}$, and stirred vigorously for 1 h. The aqueous layer was extracted with $\mathrm{CH}_{2} \mathrm{Cl}_{2}(10 \mathrm{~mL})$, and the combined organic layers were washed with sat. aq. $\mathrm{NaCl}(10 \mathrm{~mL})$, dried over $\mathrm{MgSO}_{4}$, filtered, and concentrated. The residue was purified by column chromatography (5:95 $\rightarrow$ 7:93 EtOAc/hexane), affording $\mathbf{S} 7$ (256 mg, $1.91 \mathrm{mmol})$ in 59\% yield as a colorless oil. The analytical data were in agreement with the literature. ${ }^{17,18}$

$R_{\mathrm{f}}: 0.30$ (20:80 EtOAc/hexane).

IR (neat) $\tilde{v}_{\max }\left(\mathrm{cm}^{-1}\right): 3341,3025,2974,2924,1638,1611,1508,1443,1377,1228,1172,1056,996,915$, $820,773$.

${ }^{1} \mathrm{H}$ NMR (499.75 MHz, $\left.\mathrm{CDCl}_{3}\right): \delta 7.02(\mathrm{~d}, J=8.4 \mathrm{~Hz}, 2 \mathrm{H}, \mathrm{H} 3, \mathrm{H} 5), 6.75$ (d, $\left.J=8.4 \mathrm{~Hz}, 2 \mathrm{H}, \mathrm{H} 2, \mathrm{H} 6\right)$, 5.98-5.88 (m, $1 \mathrm{H},=\mathrm{CH}), 5.75-5.58(\mathrm{~m}, 1 \mathrm{H}, \mathrm{OH}), 5.07-5.01\left(\mathrm{~m}, 2 \mathrm{H}=\mathrm{CH}_{2}\right), 3.29\left(\mathrm{~d}, J=6.7 \mathrm{~Hz}, 2 \mathrm{H}, \mathrm{CH}_{2}\right.$ $\left.\mathrm{H} 1^{\prime}\right)$.

${ }^{13} \mathrm{C}$ NMR (125.67 MHz, $\left.\mathrm{CDCl}_{3}\right): \delta 153.6(\mathrm{C} 1), 137.9(=\mathrm{CH}), 132.5(\mathrm{C} 4), 129.9(\mathrm{C} 3, \mathrm{C} 5), 115.6\left(=\mathrm{CH}_{2}\right)$, $115.4(\mathrm{C} 2, \mathrm{C} 6), 39.4\left(\mathrm{C1}^{\prime}\right)$.

1-Allyl-4-(benzyloxy)benzene $(7)^{19}$

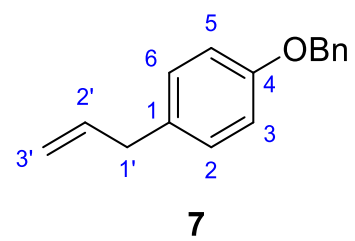

To a solution of $\mathbf{S} 7(256 \mathrm{mg}, 1.91 \mathrm{mmol})$ in anhydrous acetone $(6.8 \mathrm{~mL})$ was added $\mathrm{K}_{2} \mathrm{CO}_{3}(396 \mathrm{mg}, 2.87$ $\mathrm{mmol})$ and benzyl bromide $(295 \mu \mathrm{L}, 2.48 \mathrm{mmol})$. The reaction mixture was heated at reflux, stirred for 16 $\mathrm{h}$, cooled to rt, and filtered. The filtrate was concentrated, and the residue was dissolved in EtOAc (4.5 mL), washed with water $(4.5 \mathrm{~mL})$, sat. aq. $\mathrm{NaCl}(4.5 \mathrm{~mL})$, dried over $\mathrm{Na}_{2} \mathrm{SO}_{4}$, filtered, and concentrated. The 
residue was purified by column chromatography (1:99 $\mathrm{Et}_{2} \mathrm{O} /$ pentane), affording 7 (319 $\left.\mathrm{mg}, 1.42 \mathrm{mmol}\right)$ in $75 \%$ yield as a colorless oil. The analytical data were in agreement with the literature. ${ }^{19,20}$

$R_{\mathrm{f}}: 0.45\left(5: 95 \mathrm{Et}_{2} \mathrm{O} /\right.$ hexane) .

IR (neat) $\tilde{v}_{\max }\left(\mathrm{cm}^{-1}\right): 3031,2901,1638,1611,1583,1509,1454,1433,1380,1298,1236,1174,1111,1080$, 1023, 994, 912, 860, 807, 782, 733, 695.

${ }^{1} \mathrm{H}$ NMR (400.16 MHz, $\left.\mathrm{CDCl}_{3}\right): \delta$ 7.46-7.28 (m, $5 \mathrm{H}, 5 \times \mathrm{ArCH} \mathrm{OBn),} 7.10$ (d, J=8.8 Hz, $\left.2 \mathrm{H}, \mathrm{H} 2, \mathrm{H} 6\right)$, $6.91(\mathrm{~d}, J=8.8 \mathrm{~Hz}, 2 \mathrm{H}, \mathrm{H} 3, \mathrm{H} 5), 5.95$ (ddt, $J=16.9,10.1,6.7 \mathrm{~Hz}, 1 \mathrm{H},=\mathrm{CH}), 5.09-5.01\left(\mathrm{~m}, 4 \mathrm{H},=\mathrm{CH}_{2}\right.$, $\left.\mathrm{CH}_{2} \mathrm{OBn}\right), 3.33$ (d, $J=6.7 \mathrm{~Hz}, 2 \mathrm{H}, \mathrm{CH}_{2} \mathrm{H}^{\prime}$ ).

${ }^{13} \mathrm{C}$ NMR (100.63 MHz, $\left.\mathrm{CDCl}_{3}\right): \delta 157.3$ (C4), 137.9 (=CH), 137.2 (ArC OBn), 132.4 (C1), 129.5 (C2, C6), 128.6, 127.9, 127.5 (5× $\mathrm{ArCH} \mathrm{OBn}), 115.5\left(=\mathrm{CH}_{2}\right), 114.9(\mathrm{C} 3, \mathrm{C} 5), 70.1\left(\mathrm{CH}_{2} \mathrm{OBn}\right), 39.4\left(\mathrm{C} 1^{\prime}\right)$.

$(2 R, 3 R, 4 R, 5 R)$-Benzyl 3,4-bis(benzyloxy)-2-((benzyloxy)methyl)-5-((R,E/Z)-10-((4-benzyloxy)phenyl)1-hydroxydec-8-en-1-yl)pyrrolidine-1-carboxylate ((E/Z)-5)
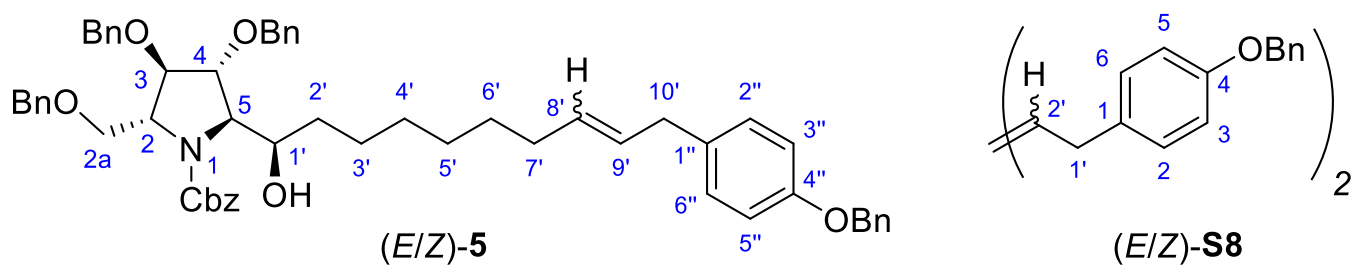

Compounds 6a (69.5 mg, $0.103 \mathrm{mmol})$ and 7 (138 mg, $0.615 \mathrm{mmol})$ were co-evaporated with DCE $(3 \times 2$ $\mathrm{mL})$ and dissolved in anhydrous, degassed $\mathrm{CH}_{2} \mathrm{Cl}_{2}(2.1 \mathrm{~mL})$, followed by the addition of Grubbs' firstgeneration catalyst $(8.5 \mathrm{mg}, 0.0103 \mathrm{mmol})$. The reaction mixture was heated at reflux for $7 \mathrm{~h}$, cooled to $\mathrm{rt}$, and concentrated. The mixture was suspended in $\mathrm{Et}_{2} \mathrm{O}(4 \mathrm{~mL})$, resulting in precipitation of $(E / Z)-\mathbf{S 8}$ as a white crystalline solid, which was removed by filtration. The filtrate was concentrated and left exposed to the atmosphere for $2 \mathrm{~d}$. The black residue was purified twice by column chromatography $(0: 100 \rightarrow 20: 80$ EtOAc/hexane), affording (E/Z)-5 (59.2 $\mathrm{mg}, 0.0677 \mathrm{mmol}, 3.6: 1)$ in $66 \%$ yield as a yellow oil.

$R_{\mathrm{f}}: 0.25$ (20:80 EtOAc/hexane).

$[\alpha]_{\mathrm{D}}^{25}-24.8\left(c 1.04, \mathrm{CHCl}_{3}\right)$.

IR (neat) $\tilde{v}_{\max }\left(\mathrm{cm}^{-1}\right): 3458,3063,3030,2924,2854,1699,1608,1509,1497,1453,1405,1345,1236,1220$, $1175,1073,1026,967,911,819,733,695$. 
${ }^{1} \mathrm{H}$ NMR (400.16 MHz, $\mathrm{CDCl}_{3} / \mathrm{D}_{2} \mathrm{O}, 3.6: 1$ mixture of (E/Z)-isomers, 1.5:1 mixture of rotamers): $\delta$ 7.457.13 (m, 25 H, 25×ArCH OBn/Cbz), 7.09 (d, $J=8.7$ Hz, 2 H, H2", H6"), 6.90 (d, J=8.7 Hz, 2 H, H3", H5"), 5.59-5.41 (m, 2 H, H8', H9'), 5.18, 5.17 (2×d, J=12.3, 12.3 Hz, 1 H, CHHCbz), 5.09-5.00 (m, 3 H, $\mathrm{CH} H \mathrm{Cbz}, \mathrm{CH}_{2} \mathrm{OBn}\left(\mathrm{C}^{\prime \prime}\right)$ ), 4.67-4.11 (m, 8.60 H, 3× $\left.\mathrm{CH}_{2} \mathrm{OBn}, \mathrm{H} 2, \mathrm{H} 3, \mathrm{H} 1^{\prime}\right), 4.10-3.97$ (m, 2.40 H, H4, H5, CHH H2a*), 3.90 (br, 0.40 H, H1'*), 3.69 (dd, $J=8.9,4.3$ Hz, 0.60 H, CHH H2a), 3.47 (dd, $J=10.6,8.9$ $\mathrm{Hz}, 1 \mathrm{H}, \mathrm{CH} H \mathrm{H} 2 \mathrm{a}), 3.33$ (d, $\left.J=6.3 \mathrm{~Hz}, 0.44 \mathrm{H}, \mathrm{CH}_{2}(Z)-\mathrm{H}_{10}^{\prime}\right), 3.26$ (d, J=5.9 Hz, $\left.1.56 \mathrm{H}, \mathrm{CH}_{2}(E)-\mathrm{H}_{10}^{\prime}\right)$, 2.15-2.09 (m, $\left.0.44 \mathrm{H}, \mathrm{CH}_{2}(Z)-\mathrm{H}^{\prime}\right), 2.04-1.95$ (m, $\left.1.56 \mathrm{H}, \mathrm{CH}_{2}(E)-\mathrm{H}^{\prime}\right), 1.57-1.06\left(\mathrm{~m}, 10 \mathrm{H}, 5 \times \mathrm{CH}_{2} \mathrm{H} 2^{\prime}\right.$, $\left.\mathrm{H} 3^{\prime}, \mathrm{H} 4^{\prime}, \mathrm{H} 5^{\prime}, \mathrm{H6} 6^{\prime}\right)$.

${ }^{13} \mathrm{C}$ NMR (100.63 MHz, $\mathrm{CDCl}_{3} / \mathrm{D}_{2} \mathrm{O}, 3.6: 1$ mixture of (E/Z)-isomers, 1.5:1 mixture of rotamers): $\delta 157.2$ $\left(\mathrm{C} 4{ }^{\prime \prime}\right), 154.9,154.7^{*}(\mathrm{C}=\mathrm{O}), 138.2,137.4,137.3,136.8,136.6^{*}, 136.4,136.2 *(5 \times \mathrm{ArC} \mathrm{OBn} / \mathrm{Cbz}), 133.63$, 133.56* (C1"), 131.9 ((E)-C9'), 131.8* ((Z)-C9'), 129.5 (C2", C6"), 129.24 ((Z)-C8'), $129.18\left((E)-\mathrm{C} 8^{\prime}\right)$, $128.7,128.65,128.6,128.5,128.3,128.25,128.2$, 128.1 128.0, 127.8, 127.7, 127.6 (25×ArCH OBn/Cbz), 115.0 ((Z)-C3", (Z)-C5"), 114.9 ((E)-C3", (E)-C5"), 81.8 (C4), 81.4, 80.1* (C3), 73.2, 71.4, 71.3 $\left(3 \times \mathrm{CH}_{2} \mathrm{OBn}\right), 70.3^{*}, 70.14(\mathrm{C} 5), 70.17\left(\mathrm{CH}_{2} \mathrm{OBn}\left(\mathrm{C} 4{ }^{\prime \prime}\right)\right)$, 69.6, 69.4* $\left(\mathrm{Cl}^{\prime}\right), 68.4\left(\mathrm{C}^{2} \mathrm{a}^{*}\right)$, 67.41, 67.37, 67.3 (C2a, $\left.\mathrm{CH}_{2} \mathrm{Cbz}\right), 63.4^{*}, 63.3$ (C2), 38.3 ((E)-C10'), 35.7, 34.7, 29.8, 29.7, 29.6, 29.5, 29.3, 27.3, 26.3, 26.1 $\left.\left(\mathrm{C} 2^{\prime}, \mathrm{C}^{\prime}, \mathrm{C}^{\prime}, \mathrm{C}^{\prime}, \mathrm{C}^{\prime},(Z)-\mathrm{C} 7^{\prime}\right)\right), 32.7\left((Z)-\mathrm{C} 10^{\prime}\right), 32.6\left((E)-\mathrm{C} 7^{\prime}\right)$.

HRMS (ESI) m/z: [M + Na] $]^{+}$Calcd for $\mathrm{C}_{57} \mathrm{H}_{63} \mathrm{NO}_{7} \mathrm{Na}$ 896.4502; Found 896.4518.

$(2 R, 3 R, 4 R, 5 R)$-Benzyl 3,4-bis(benzyloxy)-2-((benzyloxy)methyl)-5-((1R,8R/S,9S)-10-((4-benzyloxy)phenyl)-1,8,9-trihydroxydecyl)pyrrolidine-1-carboxylate (13a/13a')

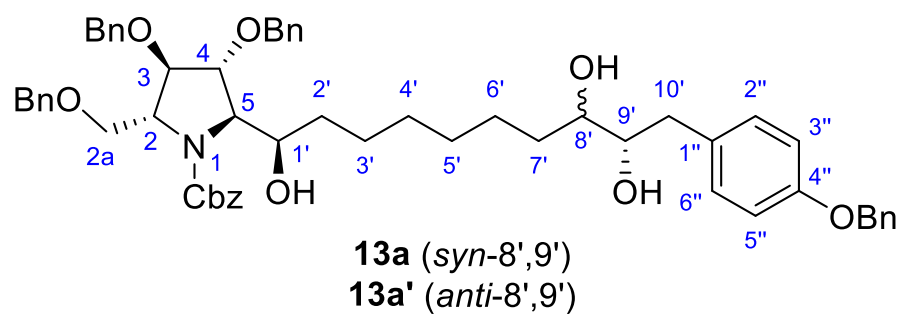

To a solution of $(E / Z)-5(43.5 \mathrm{mg}, 0.0498 \mathrm{mmol}, 4.8: 1)$ in water/t-BuOH/THF $(1.25 \mathrm{~mL}, 1: 1: 0.5 \mathrm{v} / \mathrm{v})$ at $\mathrm{rt}$ was added methanesulfonamide $(9.5 \mathrm{mg}, 0.0996 \mathrm{mmol})$ and AD-mix- $\alpha(139 \mathrm{mg})$. The reaction mixture was stirred vigorously at $\mathrm{rt}$ for $2 \mathrm{~d}$, quenched with solid $\mathrm{Na}_{2} \mathrm{SO}_{3}(150 \mathrm{mg})$, and stirred vigorously for $30 \mathrm{~min}$. The aqueous layer was extracted with EtOAc $(3 \times 2 \mathrm{~mL})$, and the combined organic layers were washed with $2 \mathrm{~N}$ aq. $\mathrm{KOH}(1 \mathrm{~mL})$, dried over $\mathrm{Na}_{2} \mathrm{SO}_{4}$, filtered, and concentrated. The residue was purified by column chromatography $(0: 100 \rightarrow 50: 50 \mathrm{EtOAc} /$ hexane $)$, affording an inseparable mixture of 13a and 13a' (39.2 mg, $0.0432 \mathrm{mmol}, 4.8: 1)$ in $87 \%$ yield as a colorless oil. 
$R_{\mathrm{f}}: 0.20$ (40:60 EtOAc/hexane).

$[\alpha]_{\mathrm{D}}^{25}-23.9\left(c 0.48, \mathrm{CHCl}_{3}\right)$.

IR (neat) $\tilde{v}_{\max }\left(\mathrm{cm}^{-1}\right): 3441,3031,2927,2856,1697,1610,1510,1454,1408,1346,1238,1176,1073,1027$, 913, 820, 735, 696 .

${ }^{1} \mathrm{H}$ NMR (400.16 MHz, $\mathrm{CDCl}_{3}$, 4.8:1 mixture of syn/anti-diols, 1.7:1 mixture of rotamers): $\delta$ 7.45-7.09 (m, 27 H, 25×ArCH OBn/Cbz, H2", H6"), 6.92 (d, J= 8.5 Hz, 2 H, H3", H5"), 5.21-5.13 (m, 1 H, CHHCbz), 5.09-4.99 (m, 3 H, CHHCbz, $\mathrm{CH}_{2} \mathrm{OBn}\left(\mathrm{C}^{\prime \prime}\right)$ ), 4.67-4.10 (m, 8.63 H, 3× $\mathrm{CH}_{2} \mathrm{OBn}, \mathrm{H} 2, \mathrm{H} 3, \mathrm{H} 1$ '), 4.09-3.97 (m, 2.27 H, H4, H5, CHH H2a*), 3.89 (br, 0.27 H, 2×H1'*), 3.78-3.71 (m, 0.17 H, anti-H9'), 3.71-3.65 (m, 0.80 H, CHH H2a, anti-H8'), 3.65-3.57 (m, $0.83 \mathrm{H}$, syn-H9'), 3.53 (d, J=6.4 Hz, 0.63 H, OH(C1')), 3.50-3.40 (m, $1.83 \mathrm{H}, \mathrm{CH} H \mathrm{H} 2 \mathrm{a}$, syn-H8'), 3.34 (d, $\left.J=6.1 \mathrm{~Hz}, 0.27 \mathrm{H}, \mathrm{OH}\left(\mathrm{C} 1^{\prime}\right) *\right), 2.86-2.75$ (m, $1 \mathrm{H}$, $\mathrm{CHH}$ syn-H10', CHH anti-H10'), 2.72-2.60 (m, 1 H, CHH syn-H10', CHH anti-H10'), 2.35-2.04 (m, 2 H, $\left.\mathrm{OH}\left(\mathrm{C}^{\prime}\right), \mathrm{OH}\left(\mathrm{C9}^{\prime}\right)\right)$, 1.61-1.03 (m, 12 H, 6× $\left.\mathrm{CH}_{2} \mathrm{H}_{2}^{\prime}, \mathrm{H}^{\prime}, \mathrm{H}^{\prime}, \mathrm{H}^{\prime}, \mathrm{H}^{\prime}, \mathrm{H}^{\prime}\right)$.

${ }^{13} \mathrm{C}$ NMR (100.63 MHz, $\mathrm{CDCl}_{3}, 4.8: 1$ mixture of syn/anti-diols, 1.7:1 mixture of rotamers): $\delta$ 157.66*, 157.63 (C4"), 154.9, 154.7* (C=O), 138.4* $138.2,137.3,137.27^{*}, 137.2,136.8,136.6^{*}, 136.4,136.2^{*}$ (5×ArC OBn/Cbz), 130.6 (C1"), 130.5, 130.45* (C2", C6"), 128.7, 128.64, 128.6, 128.5, 128.3, 128.29, 128.25, 128.2, 128.1, 128.0, 127.8, 128.77, 127.7, 127.6 (25×ArCH OBn/Cbz), 115.2*, 115.1 (C3", C5"), 81.8*, 81.7 (C4), 81.3, 80.0* (C3), 75.5 (anti-C9'), 75.2 (syn-C9'), 74.1 (anti-C8'), 73.6 (syn-C8'), 73.2, $71.3\left(3 \times \mathrm{CH}_{2} \mathrm{OBn}\right), 70.4^{*}, 70.2$ (C5), $70.1\left(\mathrm{CH}_{2} \mathrm{OBn}\left(\mathrm{C} 4{ }^{\prime \prime}\right)\right), 69.5,69.4 *\left(\mathrm{C} 1{ }^{\prime}\right), 68.3,67.4 *(\mathrm{C} 2 \mathrm{a}), 67.3$ $\left(\mathrm{CH}_{2} \mathrm{Cbz}\right), 63.4,63.2^{*}(\mathrm{C} 2), 39.4$ (syn-C10'), 36.8 (anti-C10'), 35.6*, 34.5, 33.8, 29.8*, 29.7* , 29.6, 29.5, 26.1, $25.6\left(\mathrm{C} 2^{\prime}, \mathrm{C} 3^{\prime}, \mathrm{C} 4^{\prime}, \mathrm{C} 5^{\prime}, \mathrm{C6}^{\prime}, \mathrm{C} 7^{\prime}\right)$.

HRMS (ESI) m/z: [M + Na] $]^{+}$Calcd for $\mathrm{C}_{57} \mathrm{H}_{65} \mathrm{NO}_{9} \mathrm{Na}$ 930.4557; Found 930.4564.

(2R,3R,4R,5R)-Benzyl 3,4-bis(benzyloxy)-2-((benzyloxy)methyl)-5-((1R,8R/S,9R)-10-((4-benzyloxy)phenyl)-1,8,9-trihydroxydecyl)pyrrolidine-1-carboxylate $\left(\mathbf{1 3 b} / \mathbf{1 3} \mathbf{b}^{\prime}\right)$

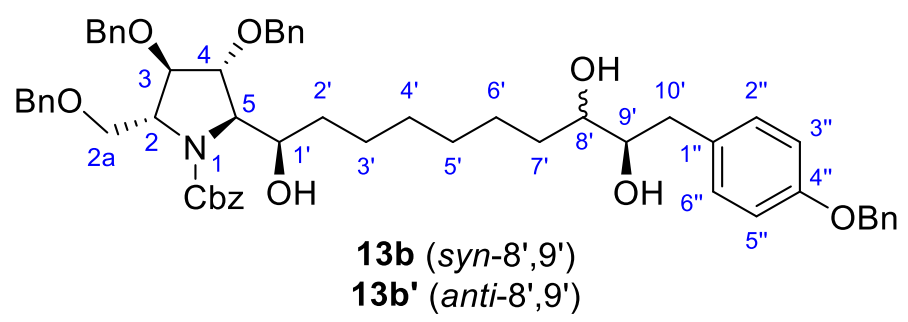


The reaction was carried out as above for 13a/13a' starting from $(E / Z)-5$ (45.8 mg, $0.0524 \mathrm{mmol}, 3.4: 1)$ using AD-mix- $\beta$, affording an inseparable mixture of $\mathbf{1 3 b}$ and $\mathbf{1 3 \mathbf { b } ^ { \prime }}$ (42.9 $\left.\mathrm{mg}, 0.0472 \mathrm{mmol}, 3.4: 1\right)$ in $90 \%$ yield as a colorless oil.

$R_{\mathrm{f}}: 0.20$ (40:60 EtOAc/hexane).

$[\alpha]_{\mathrm{D}}^{25}-17.2\left(c 0.64, \mathrm{CHCl}_{3}\right)$.

IR (neat) $\tilde{v}_{\max }\left(\mathrm{cm}^{-1}\right): 3434,3031,2927,2856,1696,1610,1510,1453,1238,1220,1176,1073,1027,912$, $819,734,696$.

${ }^{1} \mathrm{H}$ NMR (400.16 MHz, $\mathrm{CDCl}_{3}, 3.4: 1$ mixture of syn/anti-diols, 1.7:1 mixture of rotamers): $\delta$ 7.45-7.11 (m, 27 H, 25×ArCH OBn/Cbz, H2", H6"), 6.93 (d, J= 8.4 Hz, 2 H, H3", H5"), 5.21-5.14 (m, 1 H, CHHCbz), 5.10-5.01 (m, 3 H, CHHCbz, $\mathrm{CH}_{2} \mathrm{OBn}\left(\mathrm{C}^{\prime \prime}\right)$ ), 4.67-4.11 (m, 8.63 H, 3× $\left.\mathrm{CH}_{2} \mathrm{OBn}, \mathrm{H} 2, \mathrm{H} 3, \mathrm{H} 1^{\prime}\right), 4.09-3.96$ (m, 2.27 H, H4, H5, CHH H2a*), 3.89 (br, 0.27 H, H1'*), 3.79-3.72 (m, 0.23 H, anti-H9'), 3.72-3.66 (m, 0.86 H, CHH H2a, anti-H8'), 3.66-3.58 (m, 0.77 H, syn-H9'), 3.53-3.41 (m, 2.03 H, CHH H2a, syn-H8', $\left.\mathrm{OH}\left(\mathrm{Cl}^{\prime}\right)\right), 3.32\left(\mathrm{~d}, J=6.0 \mathrm{~Hz}, 0.37 \mathrm{H}, \mathrm{OH}\left(\mathrm{Cl}^{\prime}\right)^{*}\right), 2.88-2.76(\mathrm{~m}, 1 \mathrm{H}, \mathrm{CHH}$ syn-H10', CHH anti-H10'), 2.72-2.61 (m, 1 H, CHH syn-H10', CHH anti-H10'), 2.21-1.91 (m, 2 H, OH(C8'), OH(C9')), 1.57-1.07 (m, $\left.12 \mathrm{H}, 6 \times \mathrm{CH}_{2} \mathrm{H} 2^{\prime}, \mathrm{H}^{\prime}, \mathrm{H}^{\prime}, \mathrm{H}^{\prime}, \mathrm{H}^{\prime}, \mathrm{H}^{\prime}\right)$.

${ }^{13} \mathrm{C} \mathrm{NMR}\left(100.63 \mathrm{MHz}, \mathrm{CDCl}_{3}, 3.4: 1\right.$ mixture of syn/anti-diols, 1.7:1 mixture of rotamers): $\delta 157.7$ (C4"), $155.0(\mathrm{C}=\mathrm{O}), 137.4,137.2,136.8,136.8$ (5×ArC OBn/Cbz), 130.5 (C2", C6"), 128.7, 128.67, 128.6, 128.5, 128.4, 128.3, 128.27, 128.2, 128.1, 127.9, 127.8, 127.7, 127.6 (25×ArCH OBn/Cbz), 115.2, (C3", C5"), 81.9*, 81.8 (C4), 81.4, 80.0* (C3), 75.5 (anti-C9'), 75.2 (syn-C9'), 74.1 (anti-C8'), 73.7 (syn-C8'), 73.2, $71.4\left(3 \times \mathrm{CH}_{2} \mathrm{OBn}\right), 70.4 *, 70.21(\mathrm{C} 5), 70.19\left(\mathrm{CH}_{2} \mathrm{OBn}\left(\mathrm{C}^{\prime \prime}\right)\right), 69.5,69.4 *\left(\mathrm{C} 1^{\prime}\right), 68.4,67.4 *(\mathrm{C} 2 \mathrm{a}), 67.3$ $\left(\mathrm{CH}_{2} \mathrm{Cbz}\right), 63.4^{*}, 63.3$ (C2), 39.5 (syn-C10'), 36.8 (anti-C10'), 35.7*, 34.6, 33.9, 29.8*, 29.7*, 29.64, 29.56, 26.2, 25.7 (C2', C3', C4', C5', C6', C7'). Undetected: C1".

HRMS (ESI) m/z: [M + Na $]^{+}$Calcd for $\mathrm{C}_{57} \mathrm{H}_{65} \mathrm{NO}_{9} \mathrm{Na}$ 930.4557; Found 930.4554. 
$(2 R, 3 R, 4 R, 5 R)-5-((1 R, 8 S, 9 S)-1,8,9$-Trihydroxy-10-((4-hydroxy)phenyl)decyl)-2-(hydroxymethyl)pyrrolidine-3,4-diol (2a·TFA) and $(2 R, 3 R, 4 R, 5 R)-5-((1 R, 8 R, 9 S)-1,8,9$-Trihydroxy-10-((4-hydroxy)phenyl)decyl)-2-(hydroxymethyl)pyrrolidine-3,4-diol (2a' $\cdot$ TFA)
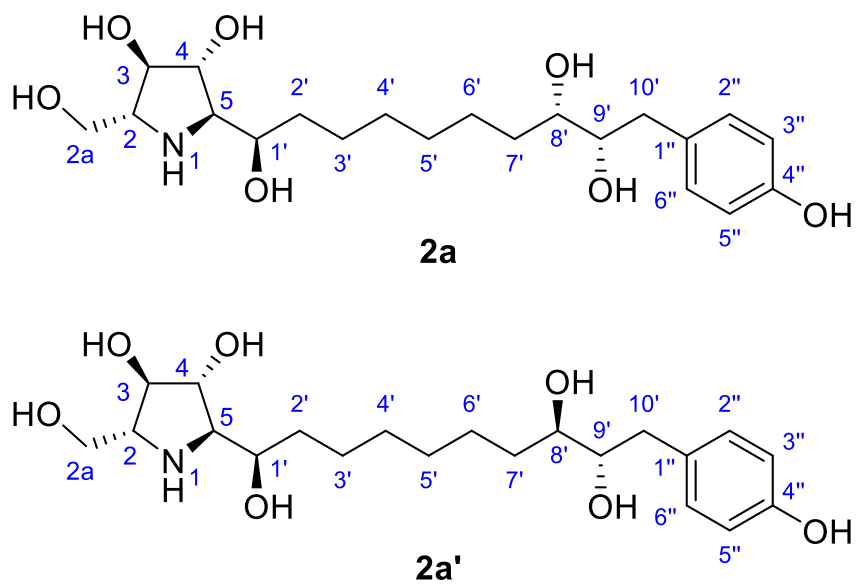

To a round-bottom flask containing a mixture of 13a and 13a' $(35.2 \mathrm{mg}, 0.0388 \mathrm{mmol}, 4.8: 1)$ was added anhydrous $\mathrm{MeOH}(2.0 \mathrm{~mL})$. After stirring to dissolve, the solution was degassed by sonication under $\mathrm{N}_{2}$ flow for $10 \mathrm{~min}$, followed by the addition of $\mathrm{PdCl}_{2}(13.8 \mathrm{mg}, 0.0776 \mathrm{mmol})$. The reaction mixture was sparged with $\mathrm{H}_{2}$ for $5 \mathrm{~min}$, then stirred at rt under an atmosphere of $\mathrm{H}_{2}$ (balloon) for $20 \mathrm{~h}$. After ESI-MS analysis confirmed complete deprotection of the starting material, the reaction mixture was filtered through a pad of Celite, rinsed in with additional $\mathrm{MeOH}(5 \mathrm{~mL})$, and concentrated. The residue was purified through a short column of RP-18 silica gel (40:60 MeOH/0.0025\% aq. TFA) affording a mixture of $2 \mathbf{a} \cdot \mathrm{TFA}$ and $\mathbf{2 a}^{\prime} \cdot$ TFA (17.8 $\left.\mathrm{mg}, 0.0337 \mathrm{mmol}, 4.8: 1\right)$ in $87 \%$ yield as a yellow lacquer. The diol mixture was further purified by semi-preparative RP-HPLC $(0 \%$ B for $0.0 \rightarrow 3.0 \mathrm{~min}$, then $10 \rightarrow 20 \%$ B for $3.0 \rightarrow 25.5 \mathrm{~min}$ ), affording $\mathbf{2 a} \cdot$ TFA $\left(R_{\mathrm{t}} 22.04 \mathrm{~min}, 9.2 \mathrm{mg}, 0.0174 \mathrm{mmol}\right)$ and $\mathbf{2 \mathbf { a } ^ { \prime }} \cdot \mathrm{TFA}\left(R_{\mathrm{t}} 19.57 \mathrm{~min}, 2.5 \mathrm{mg}, 0.0047 \mathrm{mmol}\right)$ in $45 \%$ and $12 \%$ yields, respectively, as colorless lacquers.

\section{$\underline{\mathbf{2 a} \cdot \text { TFA }}$}

$[\alpha]_{\mathrm{D}}^{25}+3.0(c 0.46, \mathrm{MeOH})$.

${ }^{1} \mathrm{H}$ NMR (400.16 MHz, CD $\left.3 \mathrm{OD}\right): \delta 7.08$ (d, J= 8.5 Hz, $\left.2 \mathrm{H}, \mathrm{H} 2 ", \mathrm{H} 6 "\right), 6.72$ (d, J=8.6 Hz, 2 H, H3", H5"), 4.26 (t, $J=6.8 \mathrm{~Hz}, 1 \mathrm{H}, \mathrm{H} 4), 3.99$ (dd, $J=8.5,6.8 \mathrm{~Hz}, 1 \mathrm{H}, \mathrm{H} 3$ ), 3.92-3.83 (m, 2 H, H1', CHH H2a), 3.80 (dd, $J=12.0,5.2 \mathrm{~Hz}, 1 \mathrm{H}, \mathrm{CH} H \mathrm{H} 2 \mathrm{a}$ ), 3.59 (ddd, $J=8.5,5.4,3.4 \mathrm{~Hz}, 1 \mathrm{H}, \mathrm{H} 9$ '), 3.45-3.38 (m, $3 \mathrm{H}, \mathrm{H} 2$, H5, H8'), 2.80 (dd, $J=13.7,5.4$ Hz, $1 \mathrm{H}, \mathrm{CHH} \mathrm{H10}$ ), 2.64 (dd, $\left.J=13.7,8.0 \mathrm{~Hz}, 1 \mathrm{H}, \mathrm{CH} H \mathrm{H} 10^{\prime}\right), 1.69-$ 1.58 (m, 2 H, $\left.\mathrm{CH}_{2} \mathrm{H} 2^{\prime}\right), 1.58-1.27$ (m, $10 \mathrm{H}, 5 \times \mathrm{CH}_{2} \mathrm{H}^{\prime}$ ', H4', H5', $\left.\mathrm{H}^{\prime}, \mathrm{H}^{\prime}\right)$. 
${ }^{1} \mathrm{H}$ NMR (499.75 MHz, $\mathrm{C}_{5} \mathrm{D}_{5} \mathrm{~N}$ ): $\delta 7.46$ (d, $J=8.5 \mathrm{~Hz}, 2 \mathrm{H}, \mathrm{H} 2 ", \mathrm{H6}$ "), 7.19 (d, J=8.5 Hz, 2 H, H3", H5"), 5.25 (t, $J=7.0 \mathrm{~Hz}, 1 \mathrm{H}, \mathrm{H} 4), 4.99$ (dd, $J=8.9,7.0 \mathrm{~Hz}, 1 \mathrm{H}, \mathrm{H} 3$ ), 4.62-4.57 (m, $1 \mathrm{H}, \mathrm{H1}$ ) ), 4.47-4.41 (m, 2 H, $\mathrm{CH}_{2} \mathrm{H} 2 \mathrm{a}$ ), 4.29-4.23 (m, 2 H, H2, H5), 4.12-4.05 (m, 1 H, H9'), 3.89-3.82 (m, 1 H, H8'), 3.25 (dd, $J=$ 13.6, 4.9 Hz, $1 \mathrm{H}, \mathrm{CHH} \mathrm{H10}$ ), 3.12 (dd, $\left.J=13.6,8.1 \mathrm{~Hz}, 1 \mathrm{H}, \mathrm{CH} H \mathrm{H} 10^{\prime}\right), 2.12-1.89$ (m, 2 H, $\mathrm{CH}_{2} \mathrm{H} 2^{\prime}$ ), 1.89-1.72 (m, 2 H, $\mathrm{CH}_{2} \mathrm{H}^{\prime}$ ), 1.72-1.10 (m, 8 H, 4× $\mathrm{CH}_{2} \mathrm{H}^{\prime}$ ', H4', H5', H6').

${ }^{13} \mathrm{C}$ NMR (100.63 MHz, CD 3 OD): $\delta 156.7$ (C4"), 131.4 (C2", C6"), 116.1 (C3", C5"), 76.7 (C3), 76.5 (C9'), $75.1(\mathrm{C} 4), 74.0\left(\mathrm{C}^{\prime}\right), 70.0\left(\mathrm{C1}^{\prime}\right), 66.0(\mathrm{C} 5), 64.8(\mathrm{C} 2), 58.5$ (C2a), $39.8\left(\mathrm{C} 10^{\prime}\right), 34.6\left(\mathrm{C} 2^{\prime}\right), 34.2$ (C7'), 30.6, 30.5, 27.0, 26.7 (C3', C4', C5', C6'). Undetected: C1".

${ }^{13} \mathrm{C}$ NMR (125.67 MHz, C5 $\left.\mathrm{D}_{5} \mathrm{~N}\right): \delta 157.7\left(\mathrm{C}^{\prime \prime}\right), 131.8$ (C2", C6"), $131.6(\mathrm{C} 1 "), 116.6(\mathrm{C} 3 ", \mathrm{C} 5 "), 77.3$ (C3), 76.6 (C9'), 75.8 (C4), 74.0 (C8'), 70.2 (C1'), 66.4 (C5), 65.3 (C2), 59.3 (C2a), $40.6\left(\mathrm{C} 10^{\prime}\right), 34.9$ (C2'), $34.8\left(\mathrm{C}^{\prime}\right), 30.6,30.4,27.1,27.0\left(\mathrm{C}^{\prime}, \mathrm{C}^{\prime}, \mathrm{C} 5^{\prime}, \mathrm{C}^{\prime}\right)$.

${ }^{19} \mathrm{~F}$ NMR (376.49 MHz, $\left.\mathrm{CD}_{3} \mathrm{OD}\right): \delta-77.0(\mathrm{~s}, \mathrm{TFA}-)$.

HRMS (ESI) m/z: $[\mathrm{M}+\mathrm{H}]^{+}$Calcd for $\mathrm{C}_{21} \mathrm{H}_{36} \mathrm{NO}_{7}$ 414.2492; Found 414.2500.

\section{$\underline{\mathbf{2 a}^{\prime} \cdot \mathrm{TFA}}$}

$[\alpha]_{\mathrm{D}}^{25}+6.8(c 0.13, \mathrm{MeOH})$.

${ }^{1} \mathrm{H}$ NMR (400.16 MHz, CD $\left.3 \mathrm{OD}\right): \delta 7.08$ (d, $\left.J=8.5 \mathrm{~Hz}, 2 \mathrm{H}, \mathrm{H} 2 ", \mathrm{H} 6 "\right), 6.72$ (d, J=8.4 Hz, $\left.2 \mathrm{H}, \mathrm{H} 3 ", \mathrm{H}^{\prime \prime}\right)$, 4.25 (br, 1 H, H4), 3.98 (br, 1 H, H3), 3.93-3.76 (m, 3 H, H1', $\mathrm{CH}_{2} \mathrm{H} 2 \mathrm{a}$ ), 3.58 (dt, J = 9.1, 4.7 Hz, 1 H, H9'), 3.47-3.36 (m, 3 H, H2, H5, H8'), 2.89 (dd, $J=14.1,3.7$ Hz, 1 H, CHH H10'), 2.54 (dd, J= 14.0, 8.9 $\left.\mathrm{Hz}, 1 \mathrm{H}, \mathrm{CH} H \mathrm{H} 10^{\prime}\right), 1.77-1.21$ (m, 12 H, 6×CH $\left.\mathrm{CH}_{2} \mathrm{H}^{\prime}, \mathrm{H} 3^{\prime}, \mathrm{H}^{\prime}, \mathrm{H}^{\prime}, \mathrm{H}^{\prime}, \mathrm{H} 7^{\prime}\right)$.

${ }^{13} \mathrm{C}$ NMR (100.63 MHz, CD 3 OD): $\delta 156.6$ (C4"), 131.6 (C1"), 131.4 (C2", C6"), 116.0 (C3", C5"), 77.5 (C9'), 76.7 (C3), 75.4 (C8'), 75.1 (C4), 70.1 (C1'), 66.0 (C5), 64.8 (C2), 58.9 (C2a), 39.4 (C10'), 33.4 (C7'), 30.7, 30.6, $26.8\left(\mathrm{C}^{\prime}, \mathrm{C}^{\prime}, \mathrm{C} 5^{\prime}, \mathrm{C6}^{\prime}\right)$. Undetected: $\mathrm{C1}^{\prime}, \mathrm{C} 2^{\prime}$.

${ }^{19} \mathrm{~F}$ NMR (376.49 MHz, $\left.\mathrm{CD}_{3} \mathrm{OD}\right): \delta-76.8(\mathrm{~s}, \mathrm{TFA}-)$.

HRMS (ESI) m/z: $[\mathrm{M}+\mathrm{H}]^{+}$Calcd for $\mathrm{C}_{21} \mathrm{H}_{36} \mathrm{NO}_{7}$ 414.2492; Found 414.2511. 
$(2 R, 3 R, 4 R, 5 R)-5-((1 R, 8 R, 9 R)-1,8,9$-Trihydroxy-10-((4-hydroxy)phenyl)decyl)-2-(hydroxymethyl)pyrrolidine-3,4-diol (2b-TFA) and $(2 R, 3 R, 4 R, 5 R)-5-((1 R, 8 S, 9 R)-1,8,9$-Trihydroxy-10-((4-hydroxy)phenyl)decyl)-2-(hydroxymethyl)pyrrolidine-3,4-diol (2 $\left.\mathbf{b}^{\prime} \cdot \mathrm{TFA}\right)$
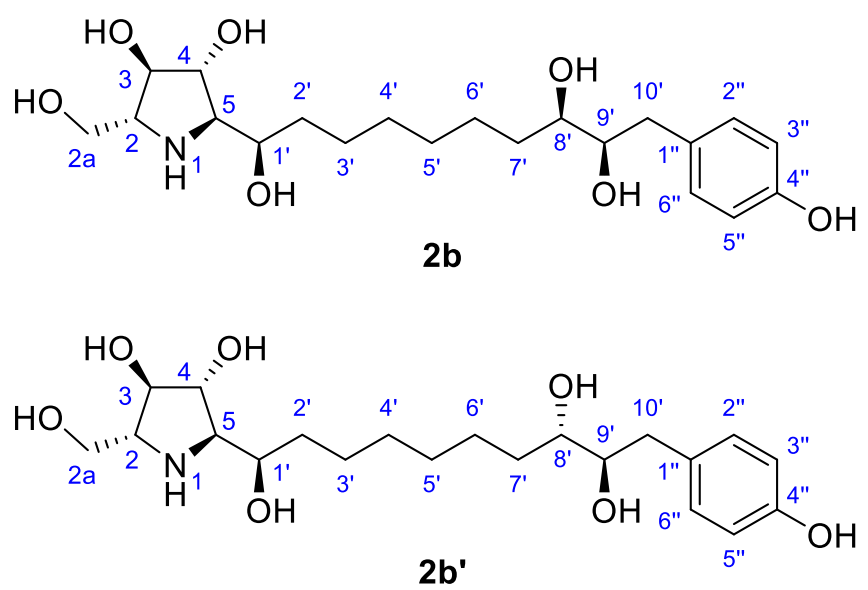

The reaction was carried out as above for $\mathbf{2} \mathbf{a} \cdot$ TFA $/ \mathbf{2} \mathbf{a}^{\prime} \cdot$ TFA starting from a mixture of $\mathbf{1 3} \mathbf{b}$ and $\mathbf{1 3} \mathbf{b}^{\prime}$ (42.9 $\mathrm{mg}, 0.0472 \mathrm{mmol}, 3.4: 1)$. Initial purification of the crude residue as above afforded a mixture of $\mathbf{2} \mathbf{b} \cdot$ TFA and $\mathbf{2} \mathbf{b}^{\prime} \cdot$ TFA (15.6 mg, $\left.0.0296 \mathrm{mmol}, 3.4: 1\right)$ in 63\% yield as a yellow lacquer. Purification by semi-preparative RP-HPLC as above afforded $\mathbf{2} \mathbf{b} \cdot$ TFA $\left(R_{\mathrm{t}} 23.37 \mathrm{~min}, 8.4 \mathrm{mg}, 0.0159 \mathrm{mmol}\right)$ and $\mathbf{2} \mathbf{b}^{\prime} \cdot \mathrm{TFA}\left(R_{\mathrm{t}} 20.90\right.$ min, $2.8 \mathrm{mg}, 0.0053 \mathrm{mmol}$ ) in $34 \%$ and $11 \%$ yields, respectively, as colorless lacquers.

\section{$\underline{\mathbf{2 b} \cdot \mathrm{TFA}}$}

$[\alpha]_{\mathrm{D}}^{25}+19.9(c 0.42, \mathrm{MeOH})$.

${ }^{1} \mathrm{H}$ NMR (400.16 MHz, CD $\left.{ }_{3} \mathrm{OD}\right): \delta 7.08$ (d, J= 8.5 Hz, 2 H, H2", H6"), 6.72 (d, J=8.5 Hz, 2 H, H3", H5"), 4.25 (t, $J=7.0 \mathrm{~Hz}, 1 \mathrm{H}, \mathrm{H} 4), 3.99$ (dd, $J=8.5,6.6 \mathrm{~Hz}, 1 \mathrm{H}, \mathrm{H} 3$ ), 3.92-3.83 (m, 2 H, H1', CHH H2a), 3.80 (dd, $J=12.0,5.2 \mathrm{~Hz}, 1 \mathrm{H}, \mathrm{CH} H \mathrm{H} 2 \mathrm{a}$ ), 3.59 (ddd, $J=8.5,5.4,3.4 \mathrm{~Hz}, 1 \mathrm{H}, \mathrm{H} 9$ '), 3.45-3.38 (m, $3 \mathrm{H}, \mathrm{H} 2$, H5, H8'), 2.80 (dd, $J=13.7,5.4 \mathrm{~Hz}, 1 \mathrm{H}, \mathrm{CHH} \mathrm{H10}$ ), 2.64 (dd, $\left.J=13.8,8.0 \mathrm{~Hz}, 1 \mathrm{H}, \mathrm{CH} H \mathrm{H} 10^{\prime}\right), 1.67-$ 1.59 (m, 2 H, $\left.\mathrm{CH}_{2} \mathrm{H} 2^{\prime}\right), 1.58-1.29$ (m, $10 \mathrm{H}, 5 \times \mathrm{CH}_{2} \mathrm{H}^{\prime}$ ', $\left.\mathrm{H}^{\prime}, \mathrm{H}^{\prime}, \mathrm{H}^{\prime}, \mathrm{H}^{\prime}\right)$.

${ }^{1} \mathrm{H}$ NMR (499.75 MHz, $\mathrm{C}_{5} \mathrm{D}_{5} \mathrm{~N}$ ): $\delta 7.46$ (d, J=8.6 Hz, 2H, H2", H6"), 7.21-7.13 (m, 2 H, H3", H5"), 5.22 (t, $J=7.1 \mathrm{~Hz}, 1 \mathrm{H}, \mathrm{H} 4), 4.99-4.92$ (m, $1 \mathrm{H}, \mathrm{H} 3), 4.58-4.50$ (m, $\left.1 \mathrm{H}, \mathrm{H} 1^{\prime}\right), 4.45-4.37$ (m, $\left.2 \mathrm{H}, \mathrm{CH}_{2} \mathrm{H} 2 \mathrm{a}\right)$, 4.23-4.15 (m, 2 H, H2, H5), 4.11-4.04 (m, 1 H, H9'), 3.88-3.82 (m, 1 H, H8'), 3.24 (dd, J= 13.6, 4.9 Hz, $1 \mathrm{H}, \mathrm{CHH} \mathrm{H10}$ ), 3.12 (dd, $\left.J=13.6,8.0 \mathrm{~Hz}, 1 \mathrm{H}, \mathrm{CH} H \mathrm{H} 10^{\prime}\right), 2.11-1.88$ (m, $\left.2 \mathrm{H}, \mathrm{CH}_{2} \mathrm{H} 2^{\prime}\right), 1.88-1.72$ (m, $\left.2 \mathrm{H}, \mathrm{CH}_{2} \mathrm{H}^{\prime}\right), 1.72-1.16$ (m, $\left.8 \mathrm{H}, 4 \times \mathrm{CH}_{2} \mathrm{H}^{\prime}, \mathrm{H}^{\prime}, \mathrm{H} 5^{\prime}, \mathrm{H} 6^{\prime}\right)$. 
${ }^{13} \mathrm{C}$ NMR (100.63 MHz, CD $\left.{ }_{3} \mathrm{OD}\right): \delta 156.7$ (C4"), 131.37 (C1"), 131.35 (C2", C6"), 116.1 (C3", C5"), 76.7 (C3), 76.5 (C9'), $75.1(\mathrm{C} 4), 74.0\left(\mathrm{C} 8^{\prime}\right), 70.0\left(\mathrm{C1}^{\prime}\right), 66.0(\mathrm{C} 5), 64.8(\mathrm{C} 2), 58.6(\mathrm{C} 2 \mathrm{a}), 39.7\left(\mathrm{C} 10^{\prime}\right), 34.6\left(\mathrm{C2} 2^{\prime}\right)$, $34.2\left(\mathrm{C}^{\prime}\right), 30.6,30.5,27.0,26.7\left(\mathrm{C}^{\prime}, \mathrm{C}^{\prime}, \mathrm{C} 5^{\prime}, \mathrm{C} 6^{\prime}\right)$.

${ }^{13} \mathrm{C}$ NMR (125.67 MHz, C5 $\left.\mathrm{D}_{5} \mathrm{~N}\right): \delta 157.7$ (C4"), 131.8 (C2", C6"), 131.7 (C1"), 116.6 (C3", C5"), 77.6 (C3), 76.6 (C9'), 76.2 (C4), 74.0 (C8'), 70.5 (C1'), 66.5 (C5), 65.3 (C2), 59.6 (C2a), $40.6\left(\mathrm{C} 10^{\prime}\right), 35.0$ (C2'), $34.9\left(\mathrm{C}^{\prime}\right), 30.6,30.5,27.1,27.0\left(\mathrm{C}^{\prime}, \mathrm{C}^{\prime}, \mathrm{C}^{\prime}, \mathrm{C}^{\prime}\right)$.

${ }^{19} \mathrm{~F}$ NMR (376.49 MHz, $\left.\mathrm{CD}_{3} \mathrm{OD}\right): \delta-76.9$ (s, TFA-).

HRMS (ESI) m/z: [M + H] $]^{+}$Calcd for $\mathrm{C}_{21} \mathrm{H}_{36} \mathrm{NO}_{7}$ 414.2492; Found 414.2504.

$\underline{\mathbf{2} \mathbf{b}^{\prime} \cdot \mathrm{TFA}}$

$[\alpha]_{\mathrm{D}}^{25}+11.1(c 0.14, \mathrm{MeOH})$.

${ }^{1} \mathrm{H}$ NMR (400.16 MHz, CD $\left.{ }_{3} \mathrm{OD}\right): \delta 7.08$ (d, $\left.J=8.5 \mathrm{~Hz}, 2 \mathrm{H}, \mathrm{H} 2 ", \mathrm{H} 6 "\right), 6.72$ (d, J= 8.5 Hz, 2 H, H3", H5"), 4.26 (t, $J=6.9 \mathrm{~Hz}, 1 \mathrm{H}, \mathrm{H} 4), 3.99$ (t, $J=7.5 \mathrm{~Hz}, 1 \mathrm{H}, \mathrm{H} 3$ ), 3.93-3.83 (m, $\left.2 \mathrm{H}, \mathrm{H} 1^{\prime}, \mathrm{CHH} \mathrm{H} 2 \mathrm{a}\right), 3.80$ (dd, $J$ $=12.0,5.2 \mathrm{~Hz}, 1 \mathrm{H}, \mathrm{CH} H \mathrm{H} 2 \mathrm{a}), 3.58\left(\mathrm{ddd}, J=9.1,5.7,3.7 \mathrm{~Hz}, 1 \mathrm{H}, \mathrm{H} 9^{\prime}\right), 3.45-3.38$ (m, $3 \mathrm{H}, \mathrm{H} 2$, H5, H8'), $2.89\left(\mathrm{dd}, J=14.1,3.7 \mathrm{~Hz}, 1 \mathrm{H}, \mathrm{CHH} \mathrm{H} 10^{\prime}\right), 2.54$ (dd, $\left.J=14.0,8.9 \mathrm{~Hz}, 1 \mathrm{H}, \mathrm{CH} H \mathrm{H} 10^{\prime}\right), 1.76-1.27$ (m, 12 $\left.\mathrm{H}, 6 \times \mathrm{CH}_{2} \mathrm{H} 2^{\prime}, \mathrm{H} 3^{\prime}, \mathrm{H} 4^{\prime}, \mathrm{H} 5^{\prime}, \mathrm{H} 6^{\prime}, \mathrm{H} 7^{\prime}\right)$.

${ }^{13} \mathrm{C}$ NMR (100.63 MHz, CD 3 OD): $\delta 156.6$ (C4"), 131.6 (C1"), 131.4 (C2", C6"), 116.0 (C3", C5"), 77.5 (C9'), $76.7(\mathrm{C} 3), 75.4\left(\mathrm{C}^{\prime}\right), 75.1(\mathrm{C} 4), 70.1\left(\mathrm{C1}^{\prime}\right), 66.0(\mathrm{C} 5), 64.8(\mathrm{C} 2), 58.6(\mathrm{C} 2 \mathrm{a}), 39.5\left(\mathrm{C} 10^{\prime}\right), 34.6\left(\mathrm{C} 2^{\prime}\right)$, $33.4\left(\mathrm{C}^{\prime}\right), 30.7,30.6,26.8,26.4\left(\mathrm{C}^{\prime}, \mathrm{C}^{\prime}, \mathrm{C} 5^{\prime}, \mathrm{C} 6^{\prime}\right)$.

${ }^{19} \mathrm{~F}$ NMR (376.49 MHz, CD $\left.{ }_{3} \mathrm{OD}\right): \delta-76.9(\mathrm{~s}, \mathrm{TFA}-)$.

HRMS (ESI) m/z: [M + Na $]^{+}$Calcd for $\mathrm{C}_{21} \mathrm{H}_{35} \mathrm{NO}_{7} \mathrm{Na}$ 436.2311; Found 436.2304. 
NMR Spectra and HPLC Traces 


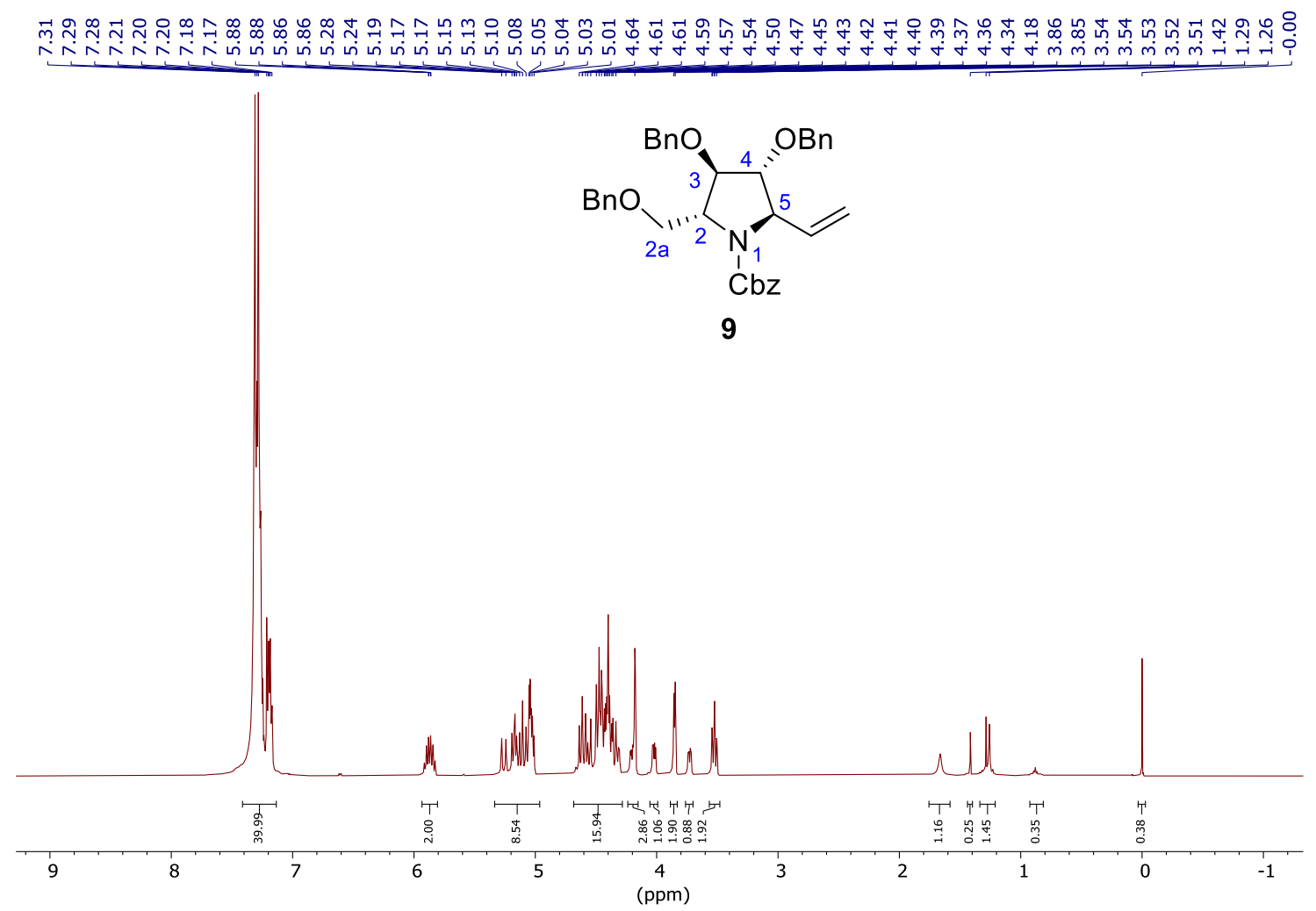

Figure S2. ${ }^{1} \mathrm{H}$ NMR spectrum $\left(499.75 \mathrm{MHz}, \mathrm{CDCl}_{3}\right)$ of 9 (1.2:1 mixture of rotamers).

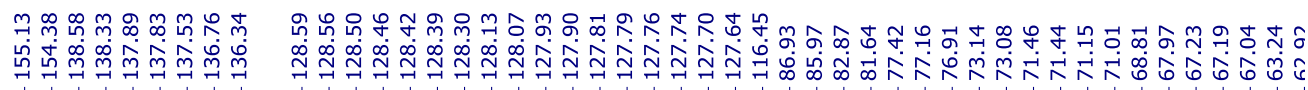

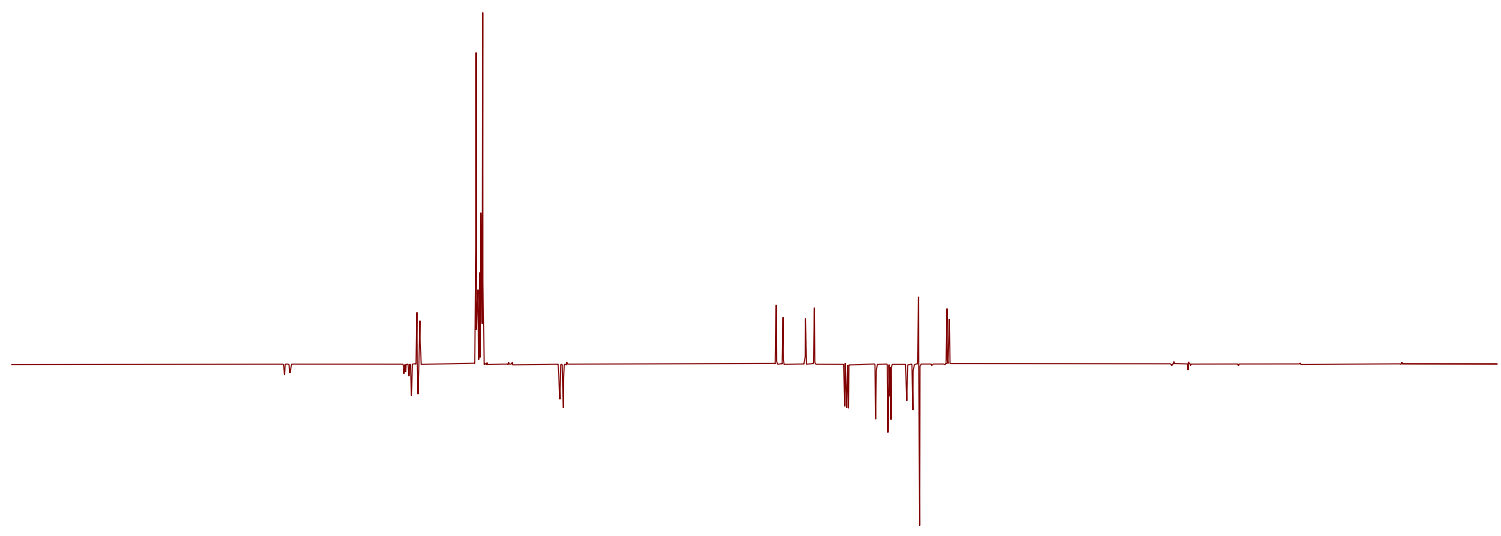

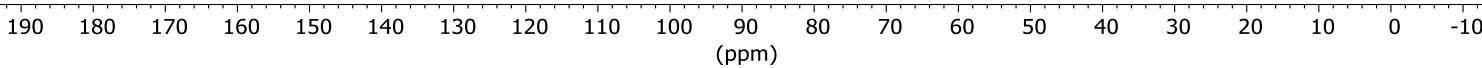

Figure S3. DEPTQ spectrum (125.67 MHz, $\left.\mathrm{CDCl}_{3}\right)$ of 9 (1.2:1 mixture of rotamers). 


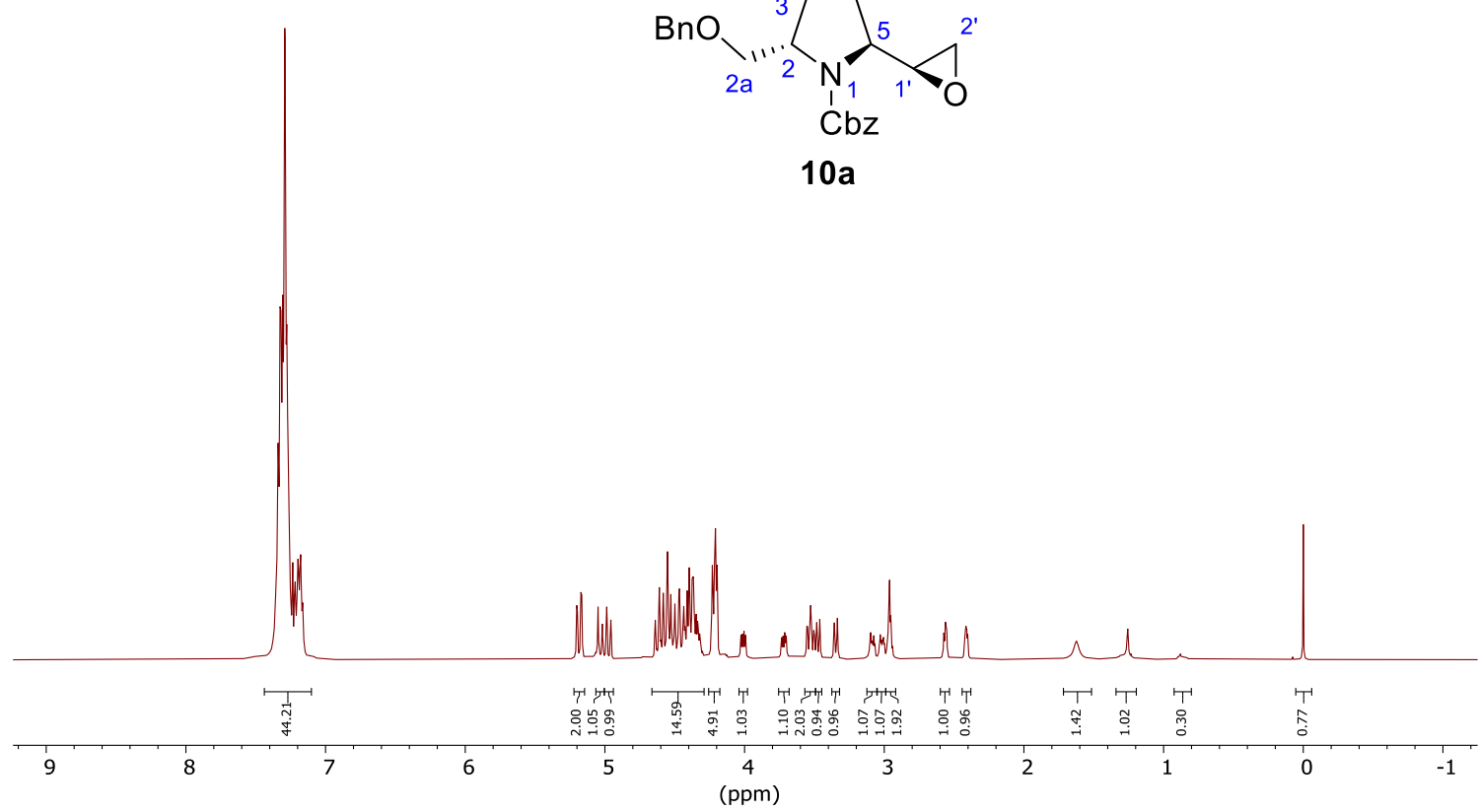

Figure S4. ${ }^{1} \mathrm{H}$ NMR spectrum $\left(400.16 \mathrm{MHz}, \mathrm{CDCl}_{3}\right)$ of $\mathbf{1 0 a}$ (1:1 mixture of rotamers).

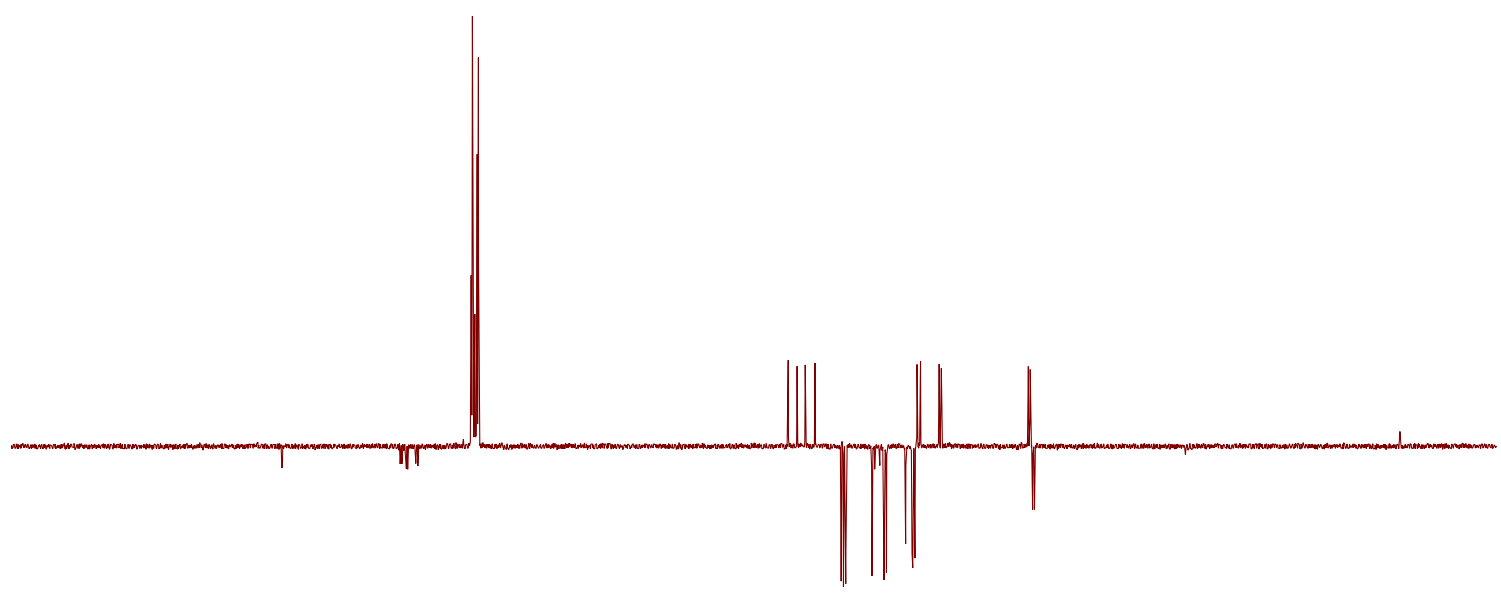

\begin{tabular}{llllllllllllllllllllll}
\hline 190 & 180 & 170 & 160 & 150 & 140 & 130 & 120 & 110 & 100 & 90 & 80 & 70 & 60 & 50 & 40 & 30 & 20 & 10 & 0 & -10
\end{tabular}

Figure S5. DEPTQ spectrum (100.63 MHz, $\left.\mathrm{CDCl}_{3}\right)$ of $\mathbf{1 0 a}(1: 1$ mixture of rotamers). 


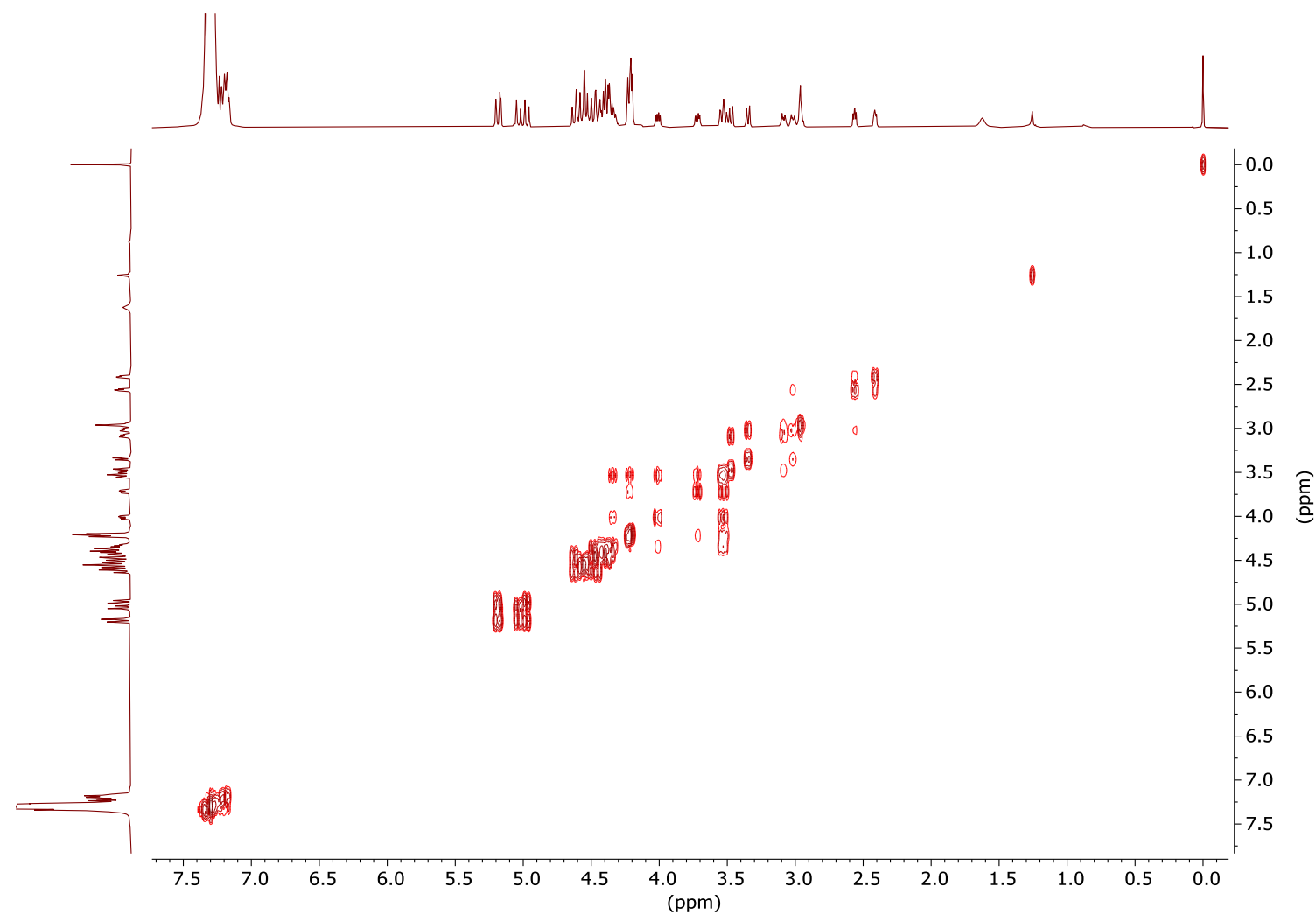

Figure S6. COSY spectrum $\left(400.16 \mathrm{MHz}, \mathrm{CDCl}_{3}\right)$ of $\mathbf{1 0 a}$.

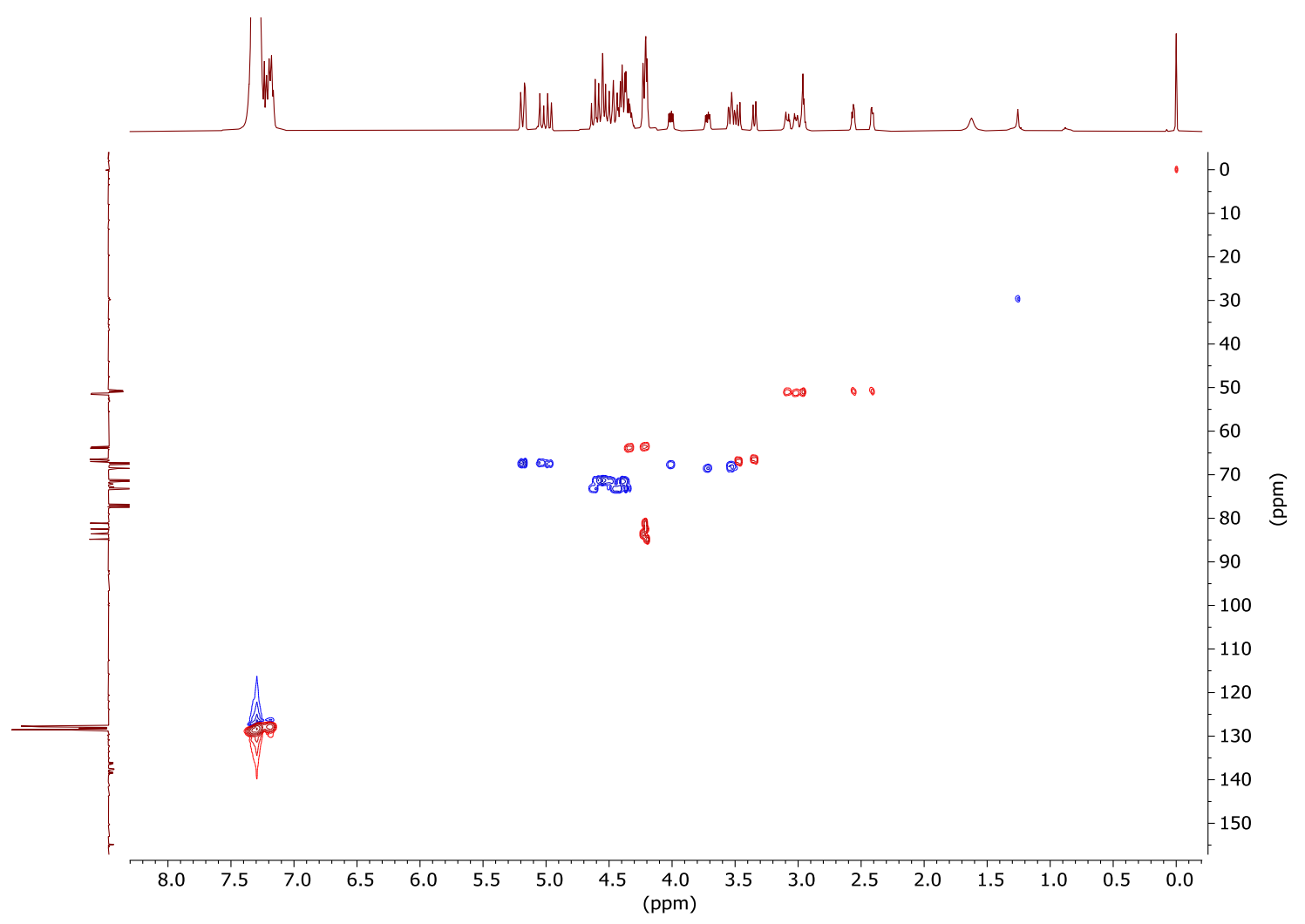

Figure S7. HSQC spectrum $\left(400.16,100.63 \mathrm{MHz}^{\left.\mathrm{CDCl}_{3}\right)}\right.$ of $\mathbf{1 0 a}$. 


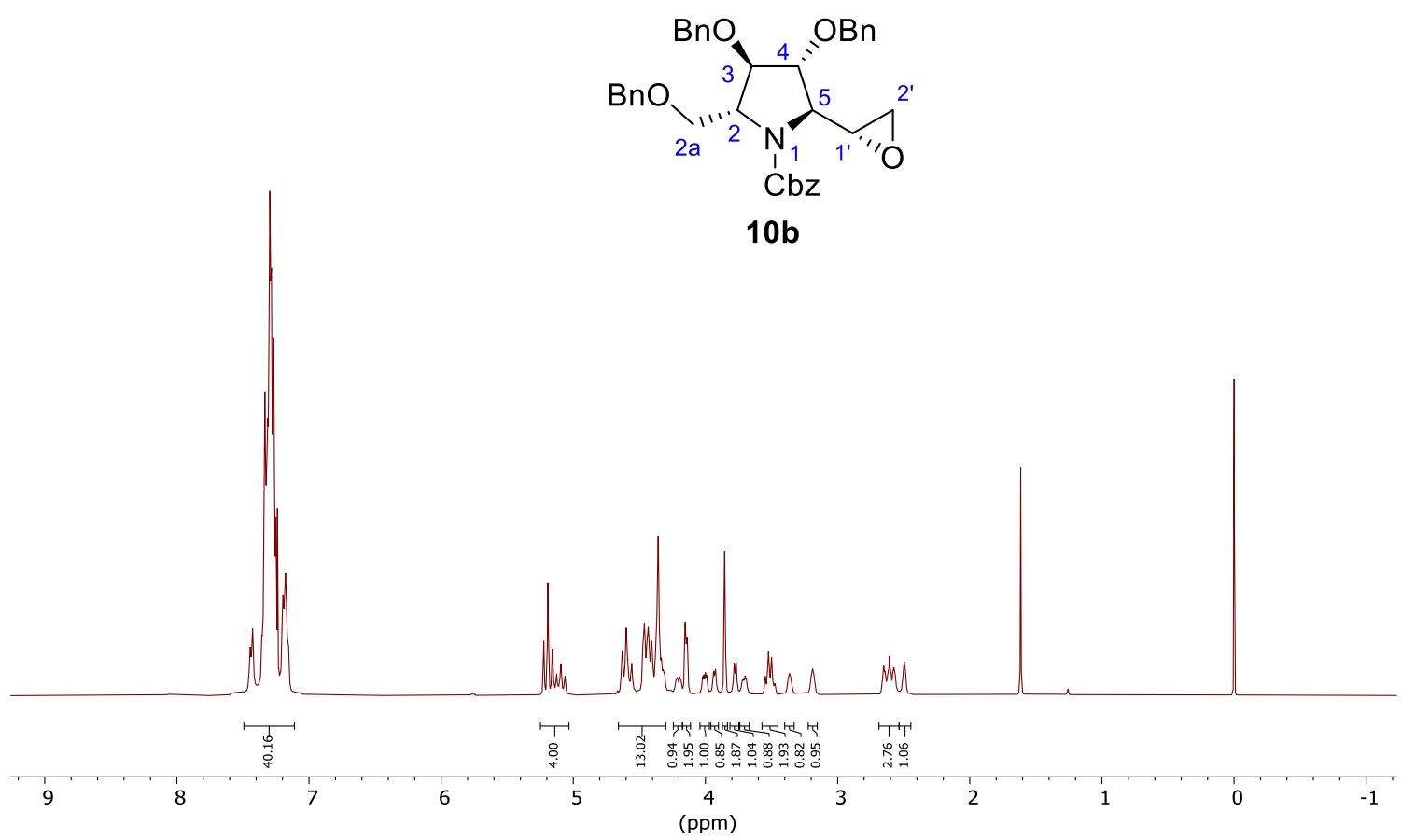

Figure S8. ${ }^{1} \mathrm{H}$ NMR spectrum $\left(400.16 \mathrm{MHz}, \mathrm{CDCl}_{3}\right)$ of $\mathbf{1 0 b}(1.2: 1$ mixture of rotamers).

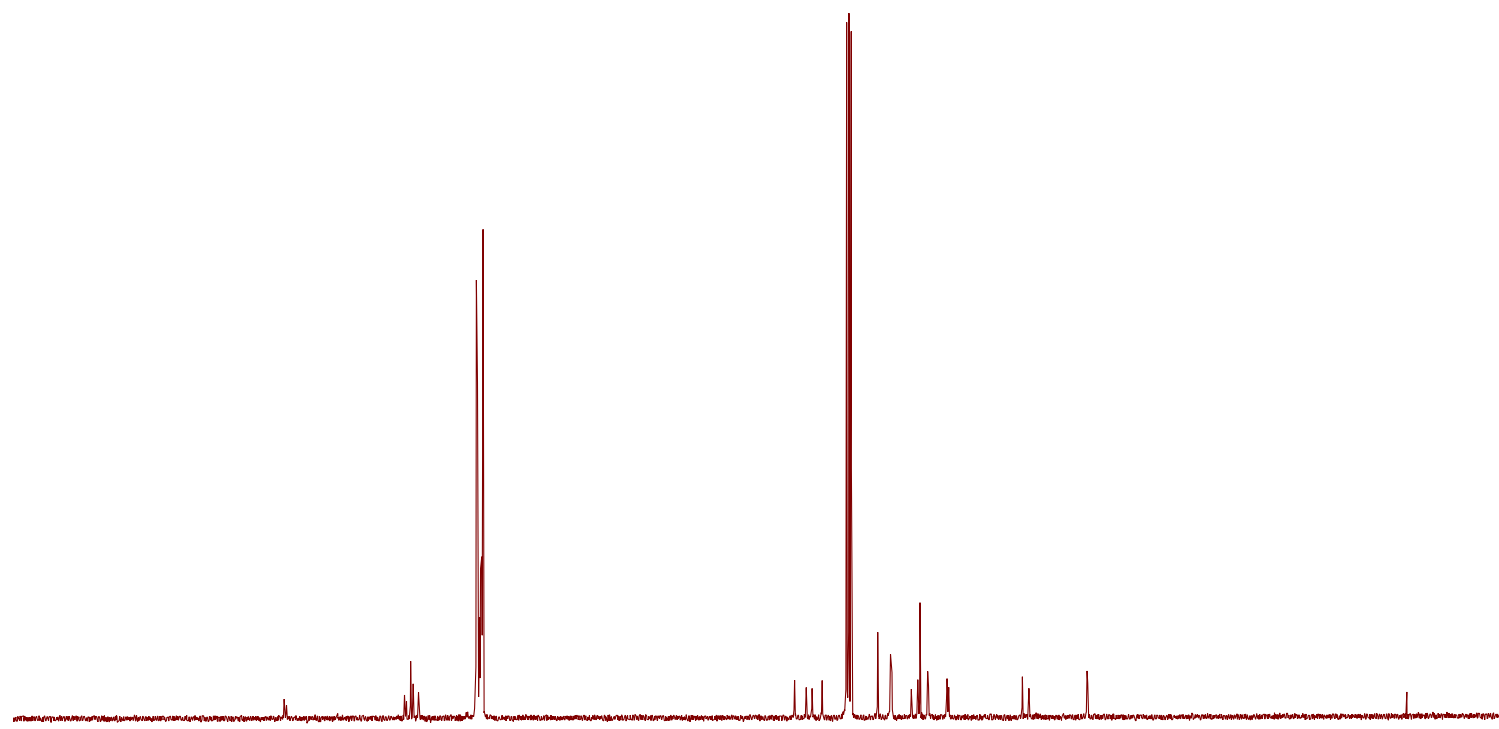

\begin{tabular}{lllllllllllllllllllllll}
\hline 190 & 180 & 170 & 160 & 150 & 140 & 130 & 120 & 110 & 100 & $\begin{array}{c}90 \\
(\mathrm{ppm})\end{array}$ & 80 & 70 & 60 & 50 & 40 & 30 & 20 & 10 & 0 & -10
\end{tabular}

Figure S9. ${ }^{13} \mathrm{C}$ NMR spectrum $\left(100.63 \mathrm{MHz}, \mathrm{CDCl}_{3}\right)$ of $\mathbf{1 0 b}(1.2: 1$ mixture of rotamers). 


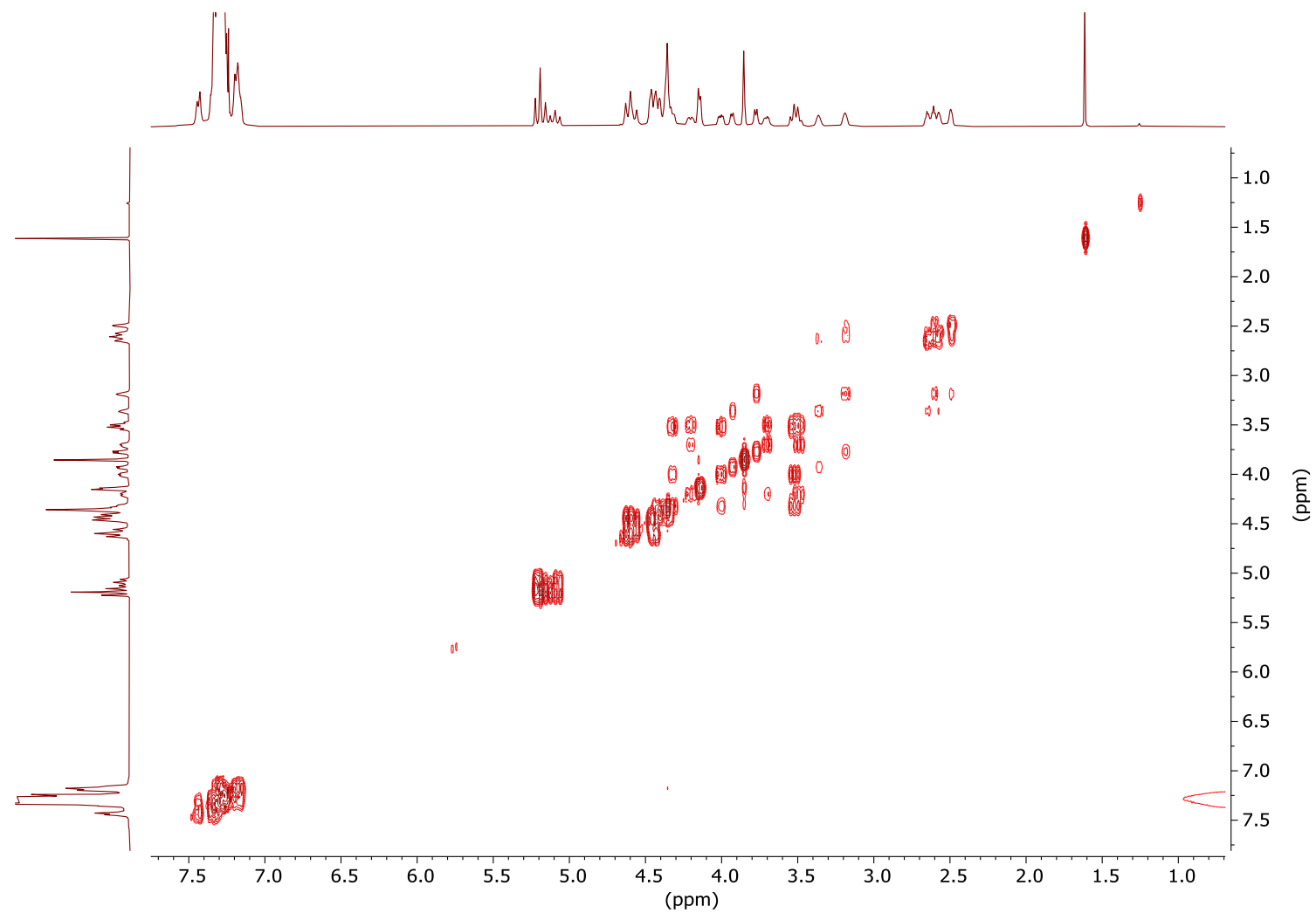

Figure S10. COSY spectrum $\left(400.16 \mathrm{MHz}, \mathrm{CDCl}_{3}\right)$ of $\mathbf{1 0 b}$.

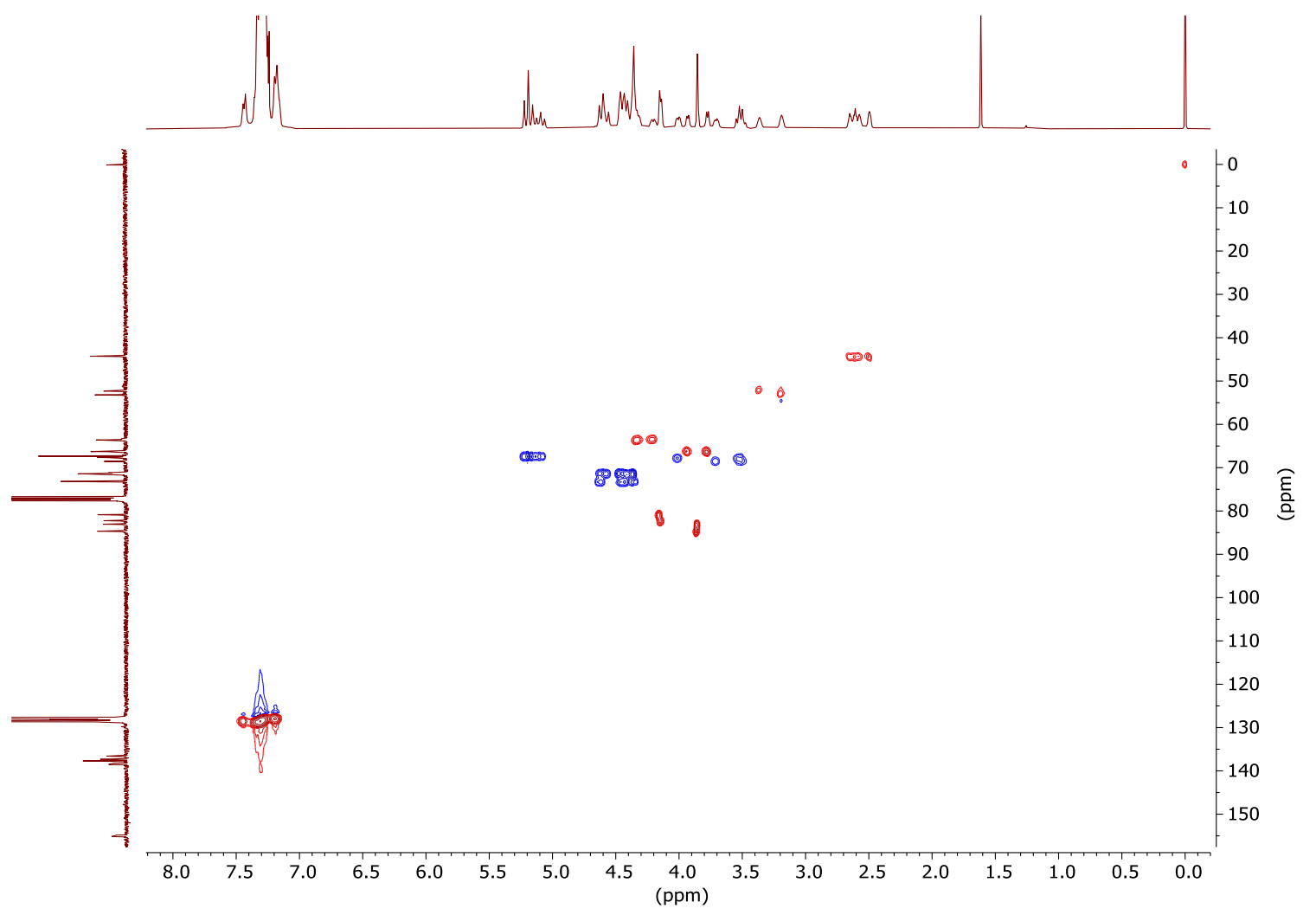

Figure S11. HSQC spectrum $\left(400.16,100.63 \mathrm{MHz}, \mathrm{CDCl}_{3}\right)$ of $\mathbf{1 0 b}$. 


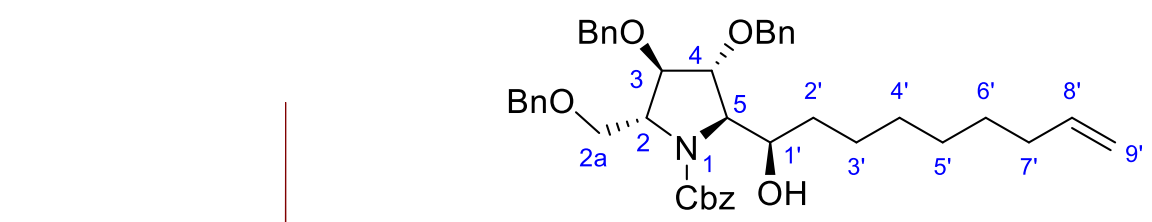

$6 \mathbf{a}$

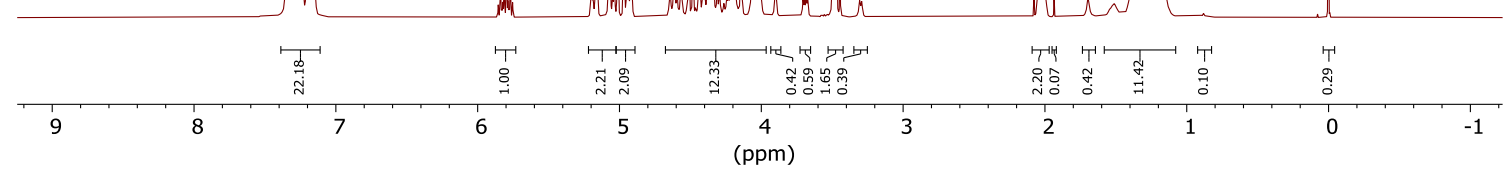

Figure S12. ${ }^{1} \mathrm{H}$ NMR spectrum $\left(400.16 \mathrm{MHz}, \mathrm{CDCl}_{3}\right)$ of $\mathbf{6 a}$ (1.6:1 mixture of rotamers).

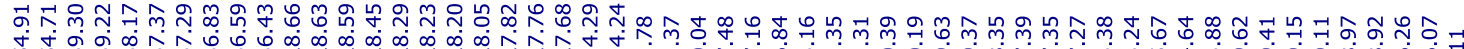

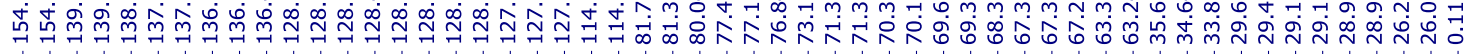

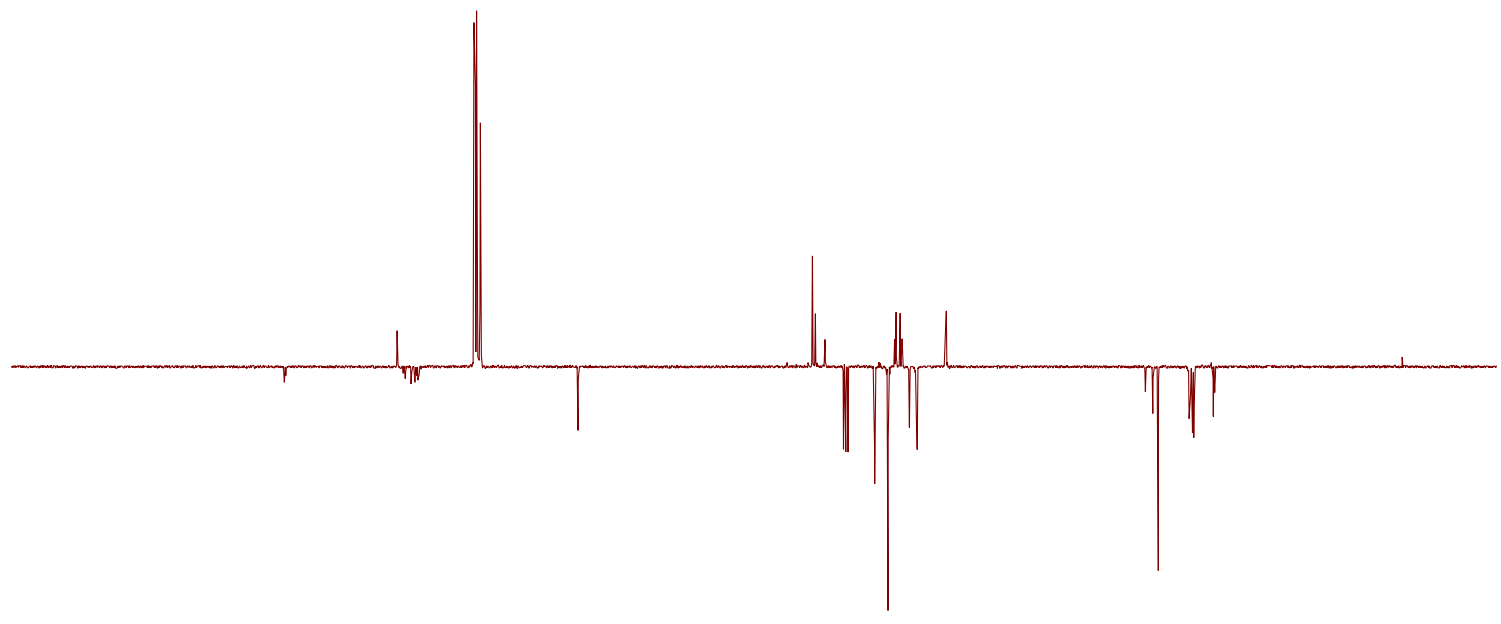

\begin{tabular}{llllllllllllllllllllll}
\hline 190 & 180 & 170 & 160 & 150 & 140 & 130 & 120 & 110 & 100 & $\begin{array}{c}90 \\
(\mathrm{ppm})\end{array}$ & 80 & 70 & 60 & 50 & 40 & 30 & 20 & 10 & 0 & -10
\end{tabular}

Figure S13. DEPTQ spectrum (100.63 MHz, $\left.\mathrm{CDCl}_{3}\right)$ of $\mathbf{6 a}$ (1.6:1 mixture of rotamers). 


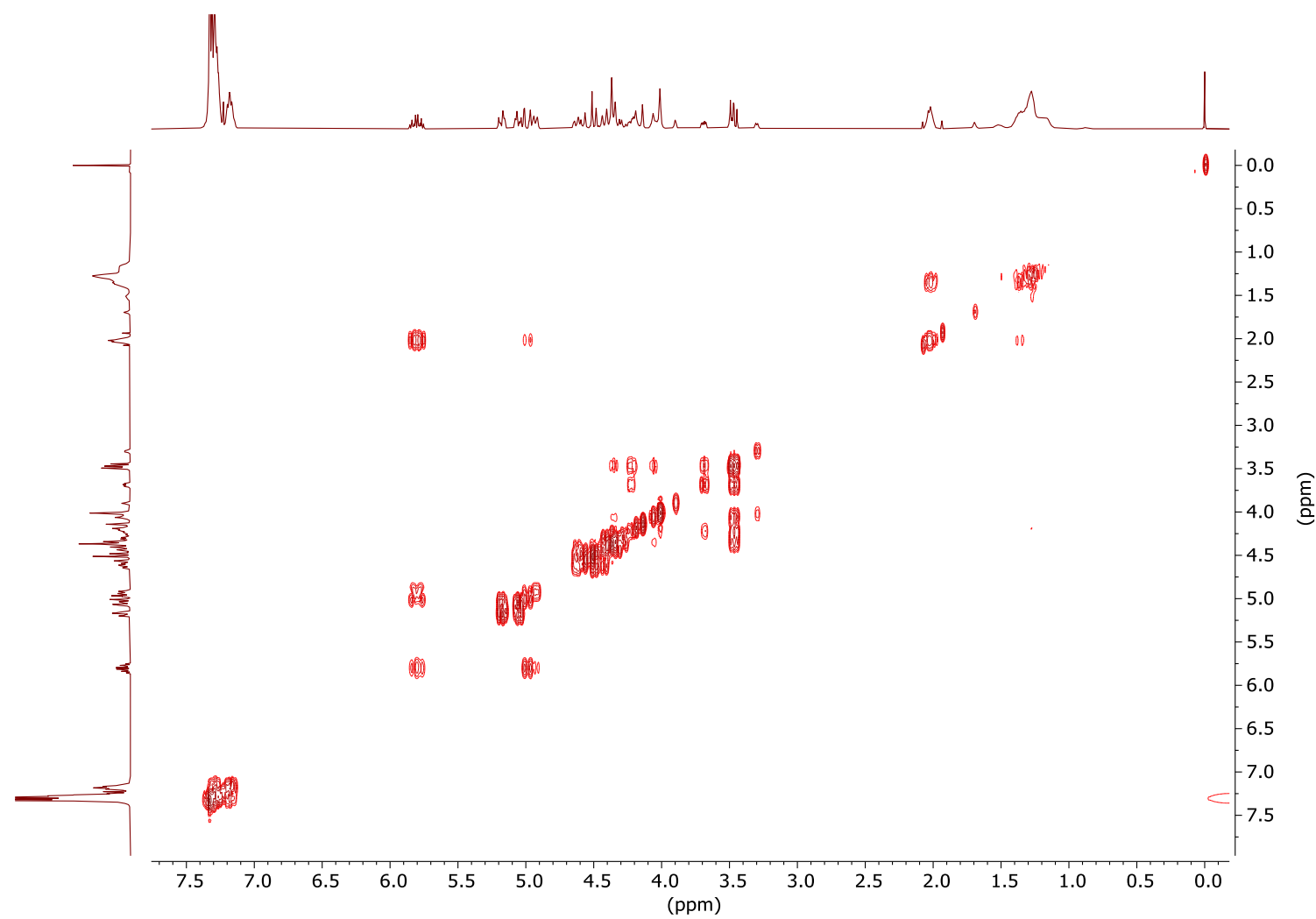

Figure S14. COSY spectrum $\left(400.16 \mathrm{MHz}, \mathrm{CDCl}_{3}\right)$ of $\mathbf{6 a}$.

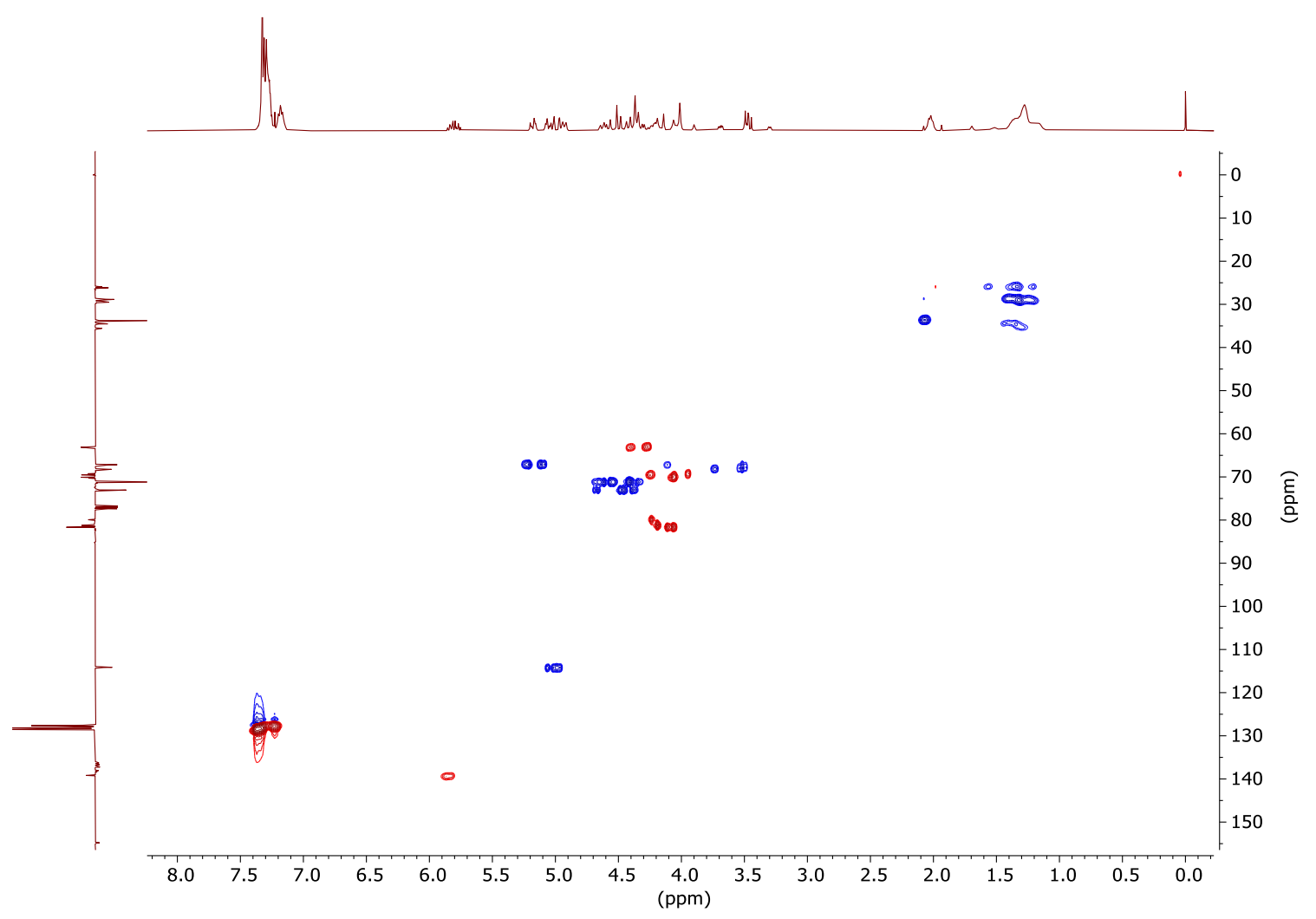

Figure S15. HSQC spectrum $\left(400.16,100.63 \mathrm{MHz}, \mathrm{CDCl}_{3}\right)$ of $6 \mathbf{a}$. 


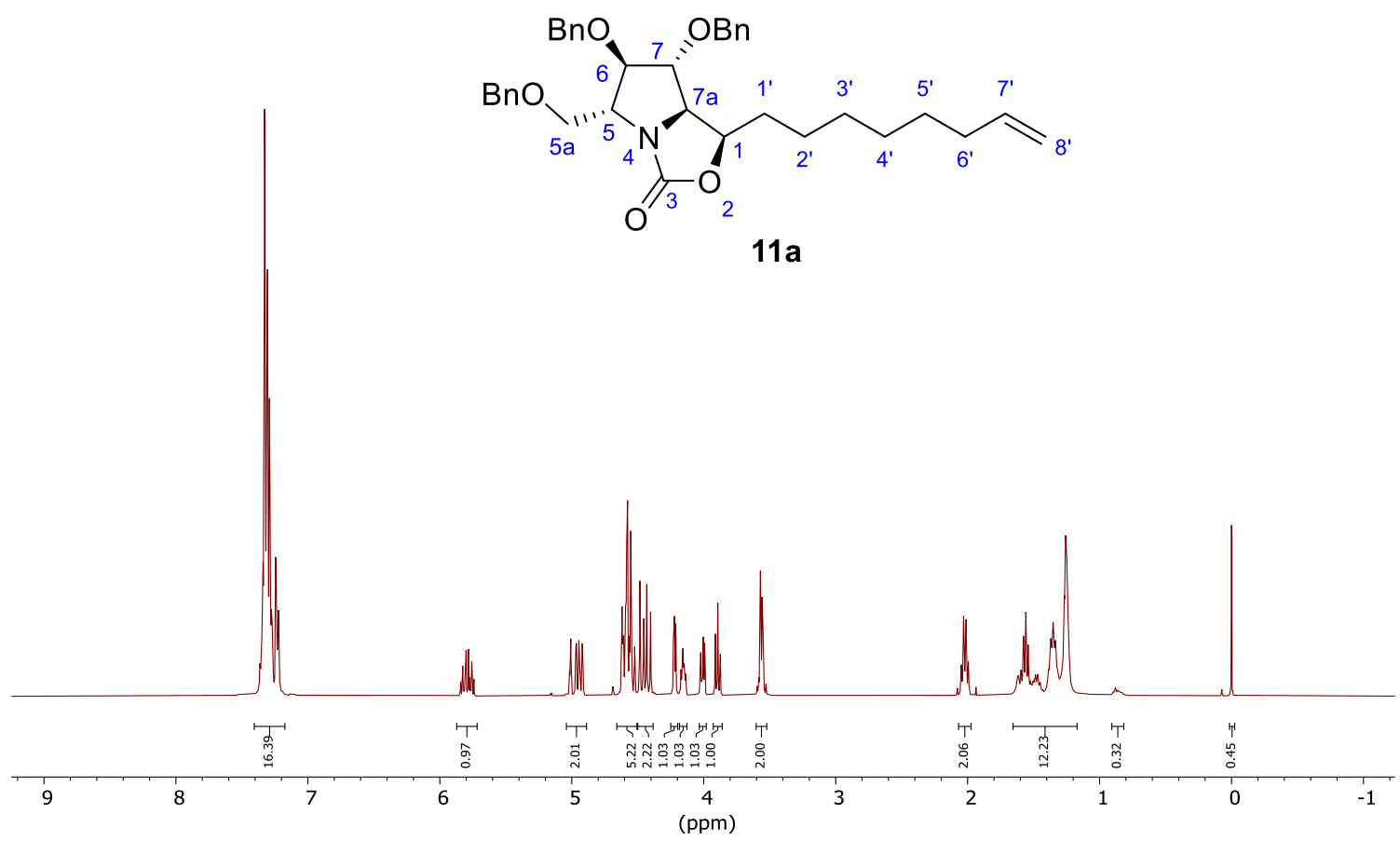

Figure S16. ${ }^{1} \mathrm{H}$ NMR spectrum $\left(400.16 \mathrm{MHz}, \mathrm{CDCl}_{3}\right)$ of $\mathbf{1 1 a}$.

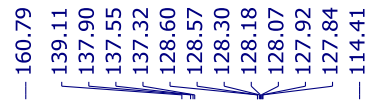

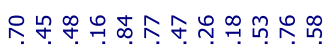

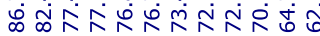

m

लं

$\longrightarrow$

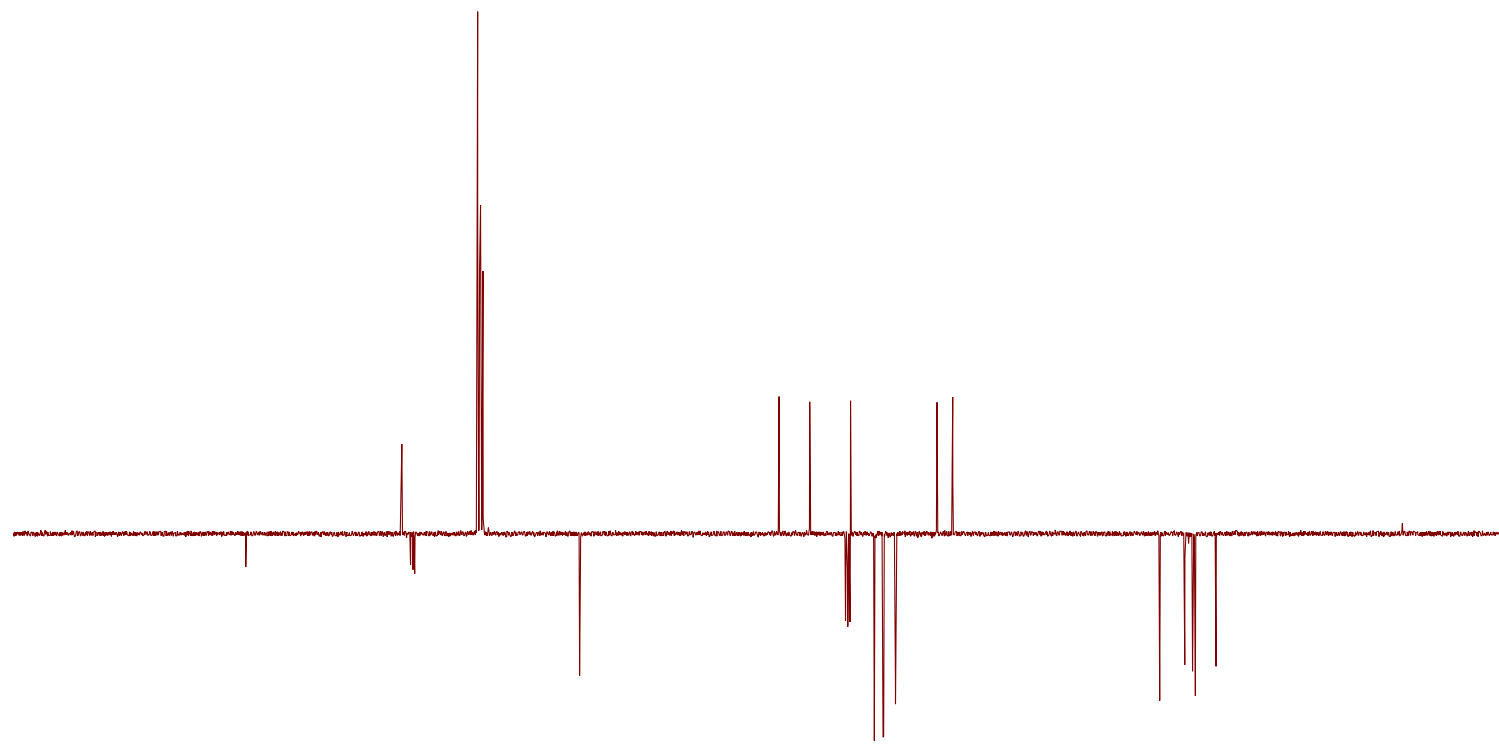

\begin{tabular}{lllllllllllllllllllll}
\hline 190 & 180 & 170 & 160 & 150 & 140 & 130 & 120 & 110 & 100 & 90 & 80 & 70 & 60 & 50 & 40 & 30 & 20 & 10 & 0 & -10 \\
$(\mathrm{ppm})$ & & & & &
\end{tabular}

Figure S17. DEPTQ spectrum (100.63 MHz, $\left.\mathrm{CDCl}_{3}\right)$ of 11a. 


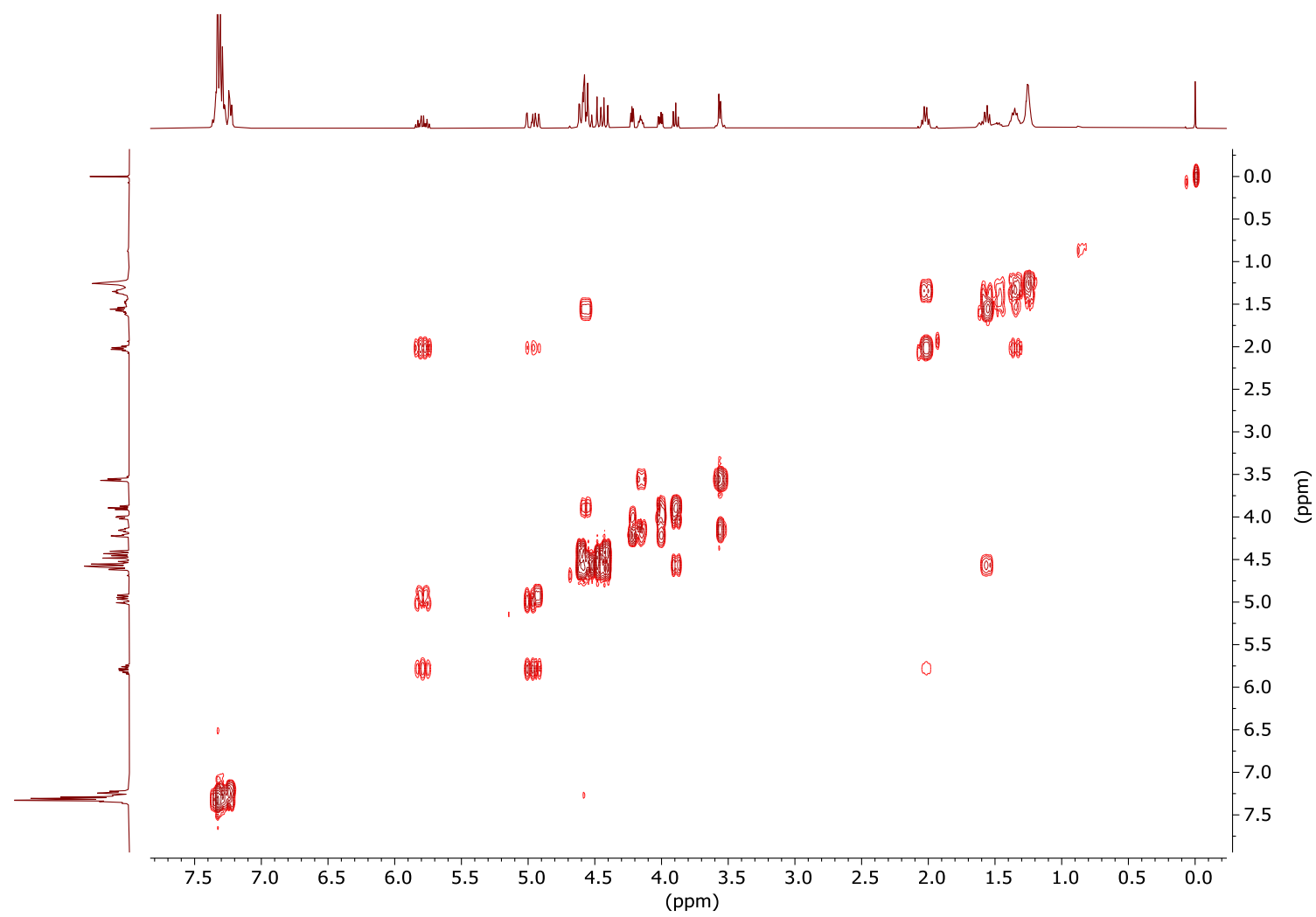

Figure S18. COSY spectrum (400.16 MHz, $\left.\mathrm{CDCl}_{3}\right)$ of $11 a$.

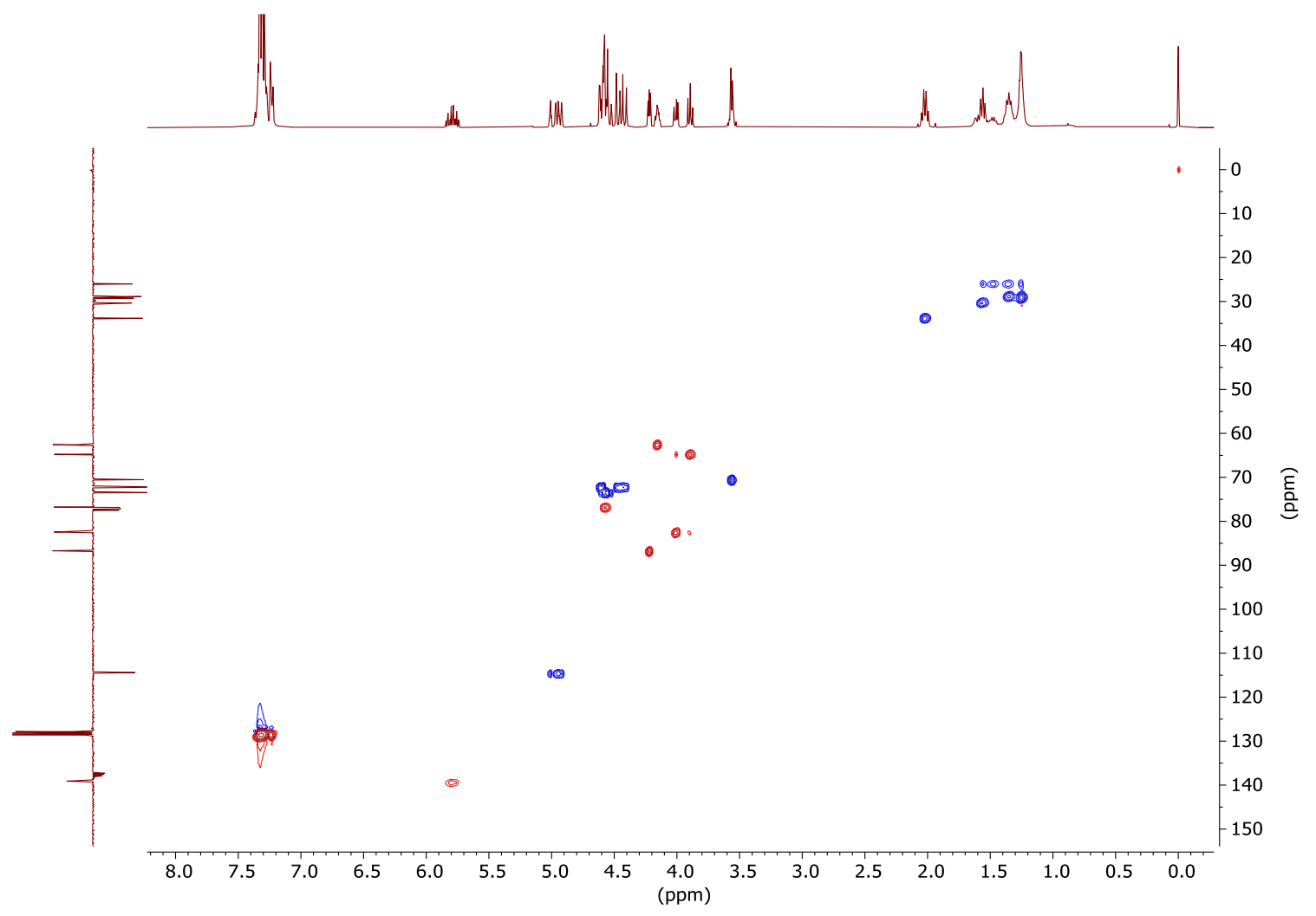

Figure S19. HSQC spectrum $\left(400.16,100.63 \mathrm{MHz}, \mathrm{CDCl}_{3}\right)$ of $\mathbf{1 1 a}$. 


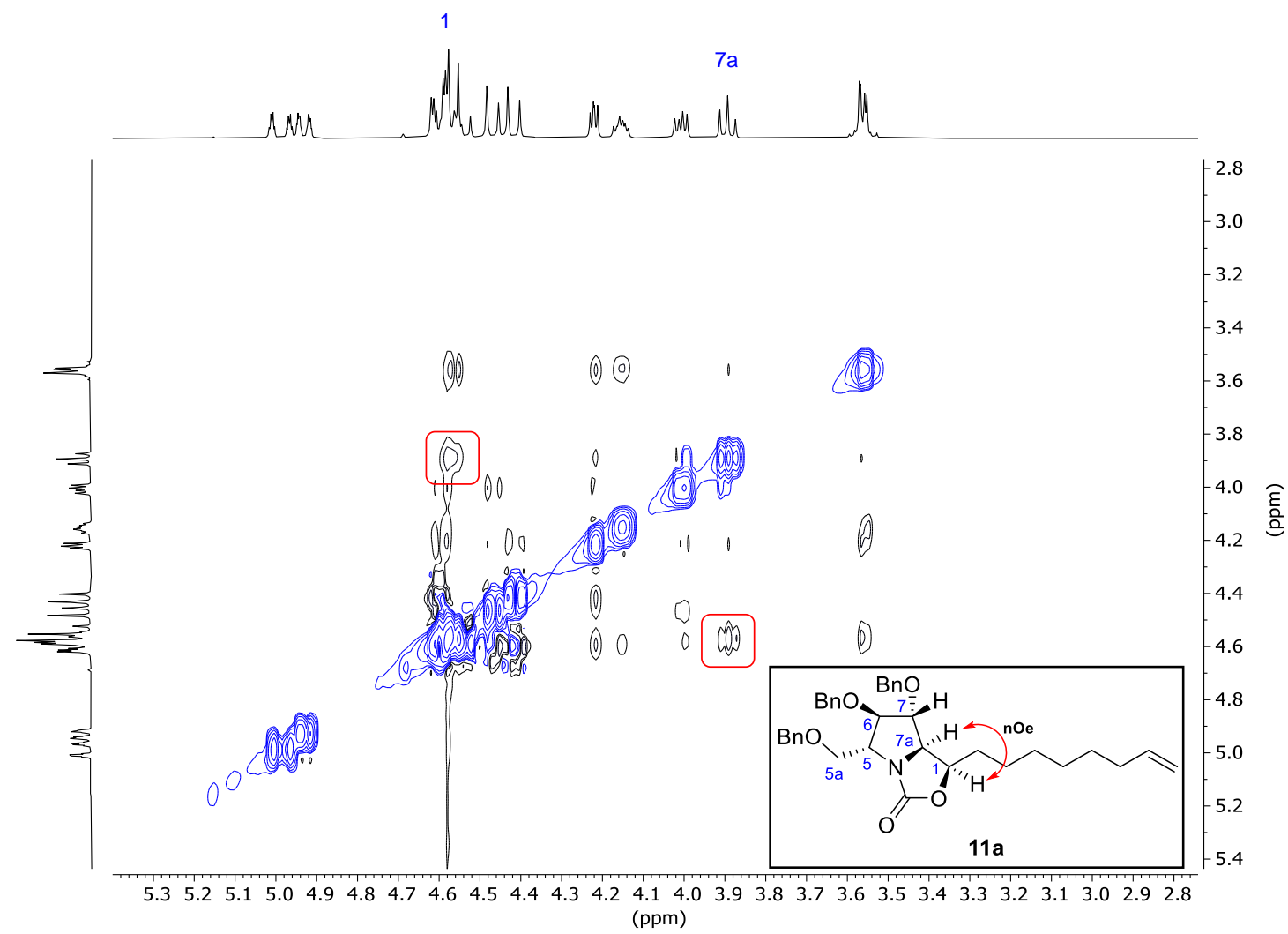

Figure S20. 2D NOESY spectrum (400.16 MHz, $\left.\mathrm{CDCl}_{3}\right)$ of $\mathbf{1 1 a}$.
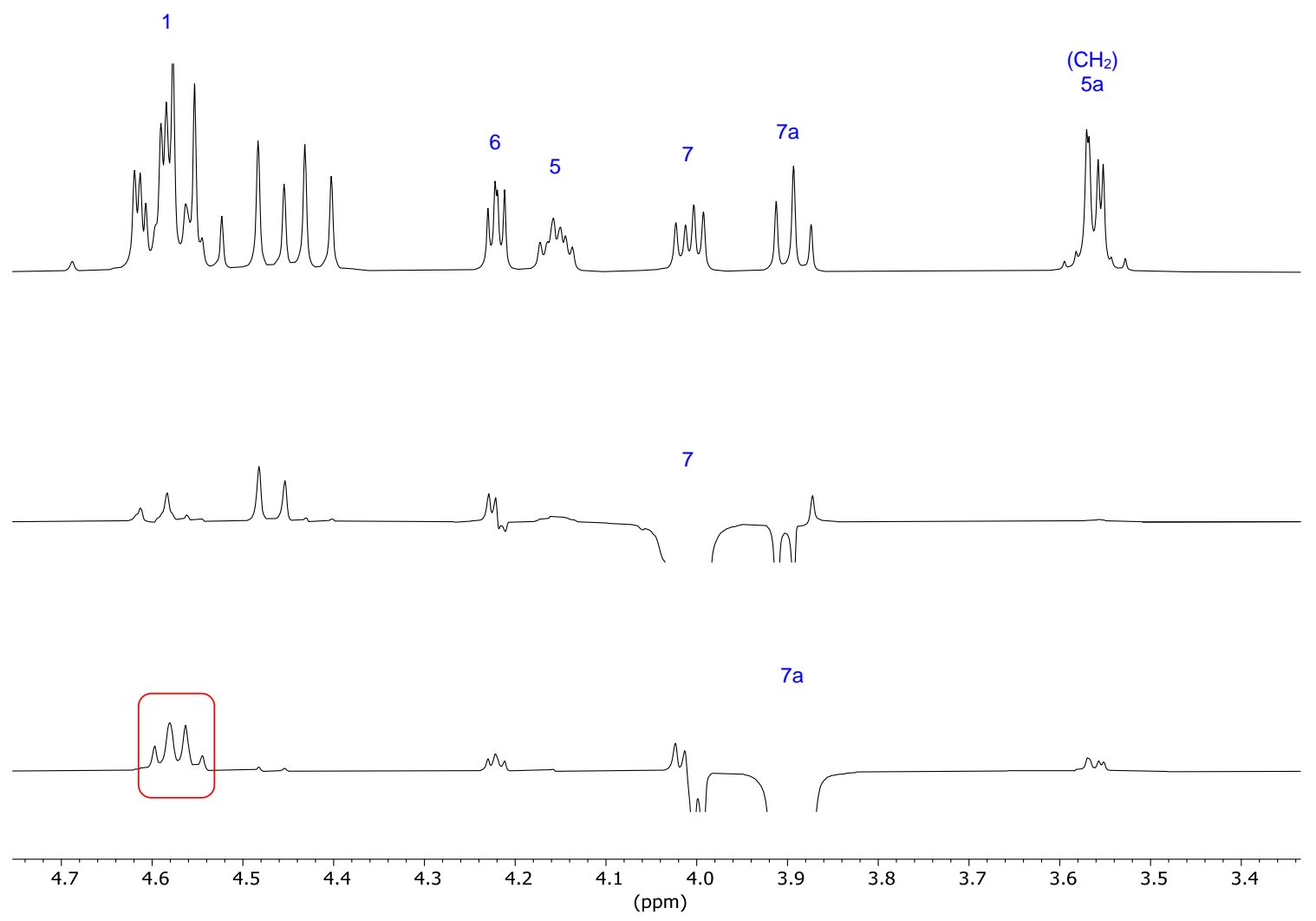

Figure S21. ${ }^{1} \mathrm{H}$ NMR and 1D NOESY spectra $\left(400.16 \mathrm{MHz}, \mathrm{CDCl}_{3}\right)$ of $\mathbf{1 1 a}$. 


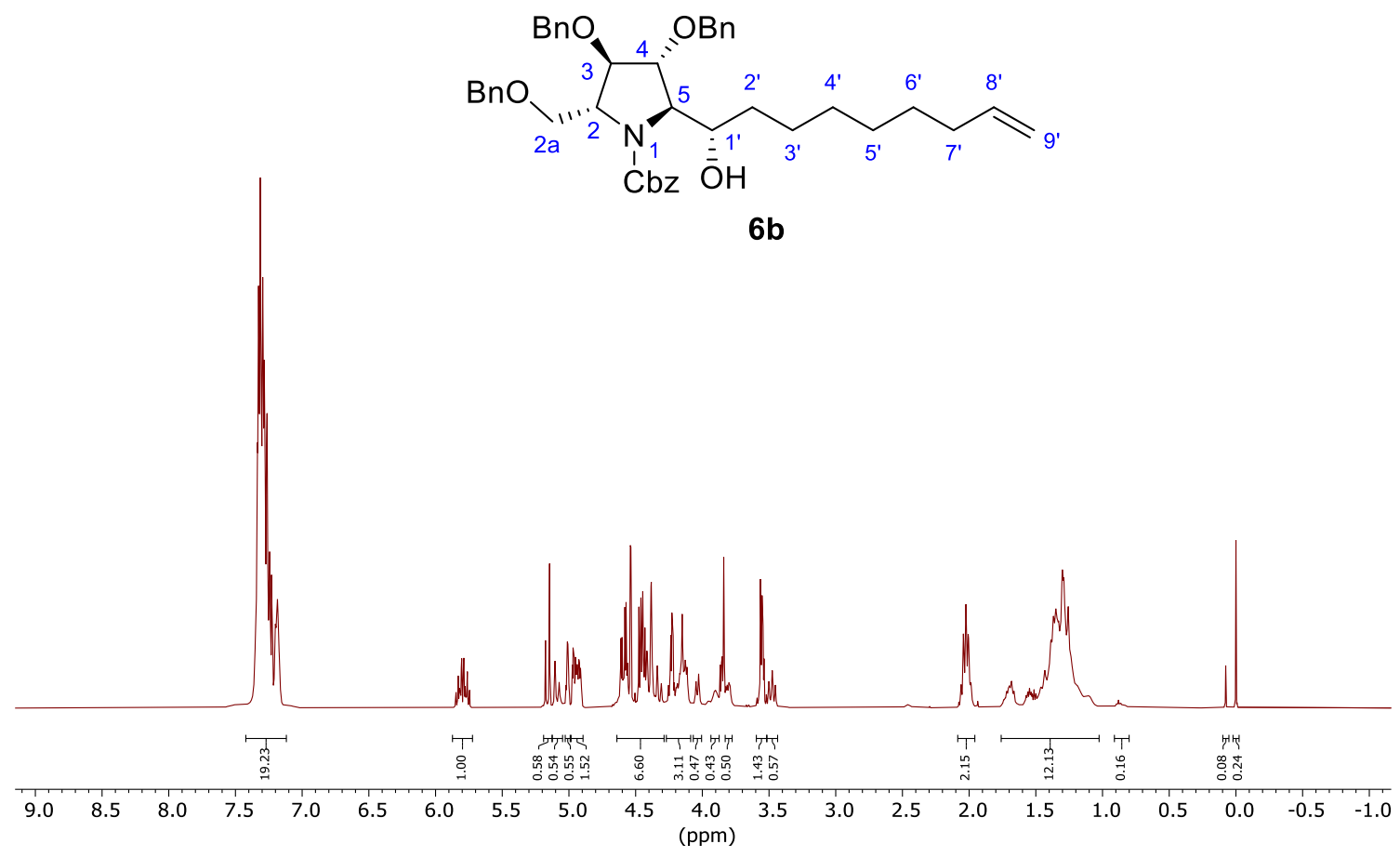

Figure S22. ${ }^{1} \mathrm{H}$ NMR spectrum $\left(400.16 \mathrm{MHz}, \mathrm{CDCl}_{3}\right)$ of $\mathbf{6 b}$ and $\mathbf{1 1 b}(1.4: 1$ mixture).

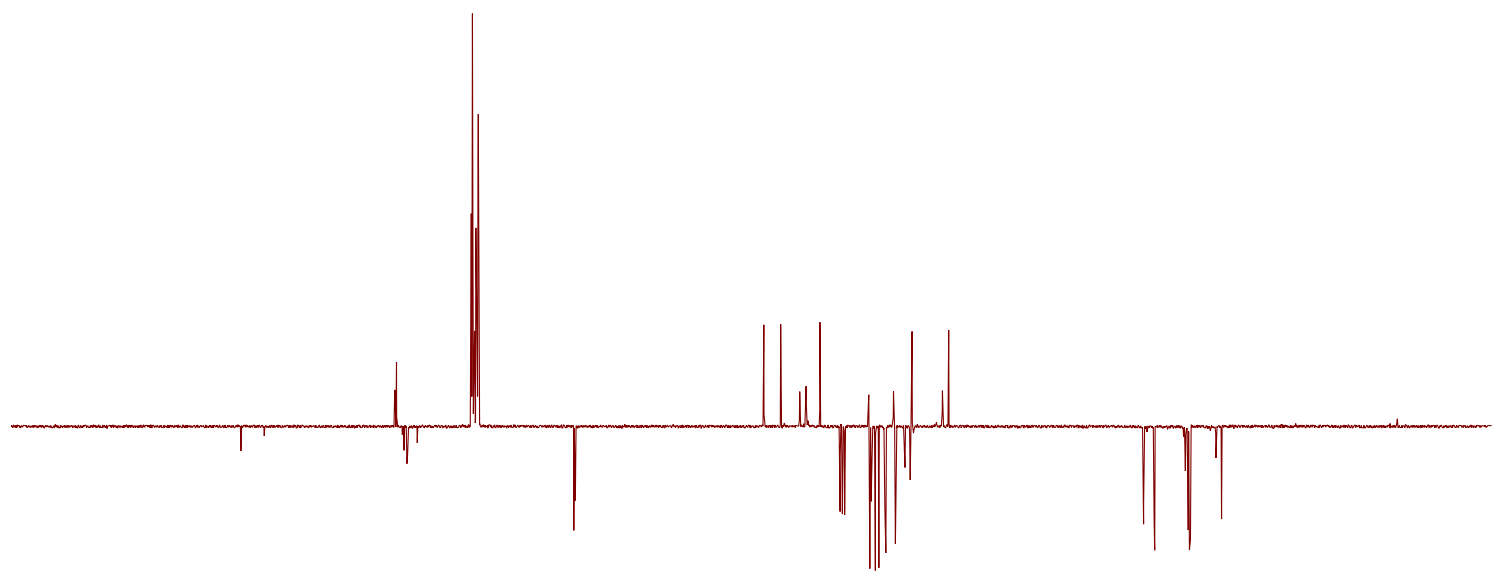

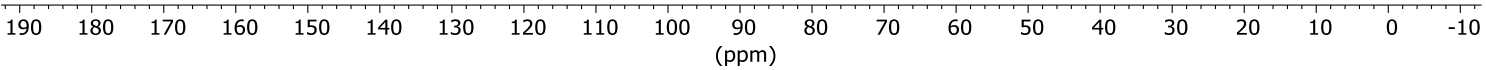

Figure S23. DEPTQ spectrum (100.63 MHz, $\left.\mathrm{CDCl}_{3}\right)$ of $\mathbf{6 b}$ and $\mathbf{1 1 b}(1.4: 1$ mixture). 


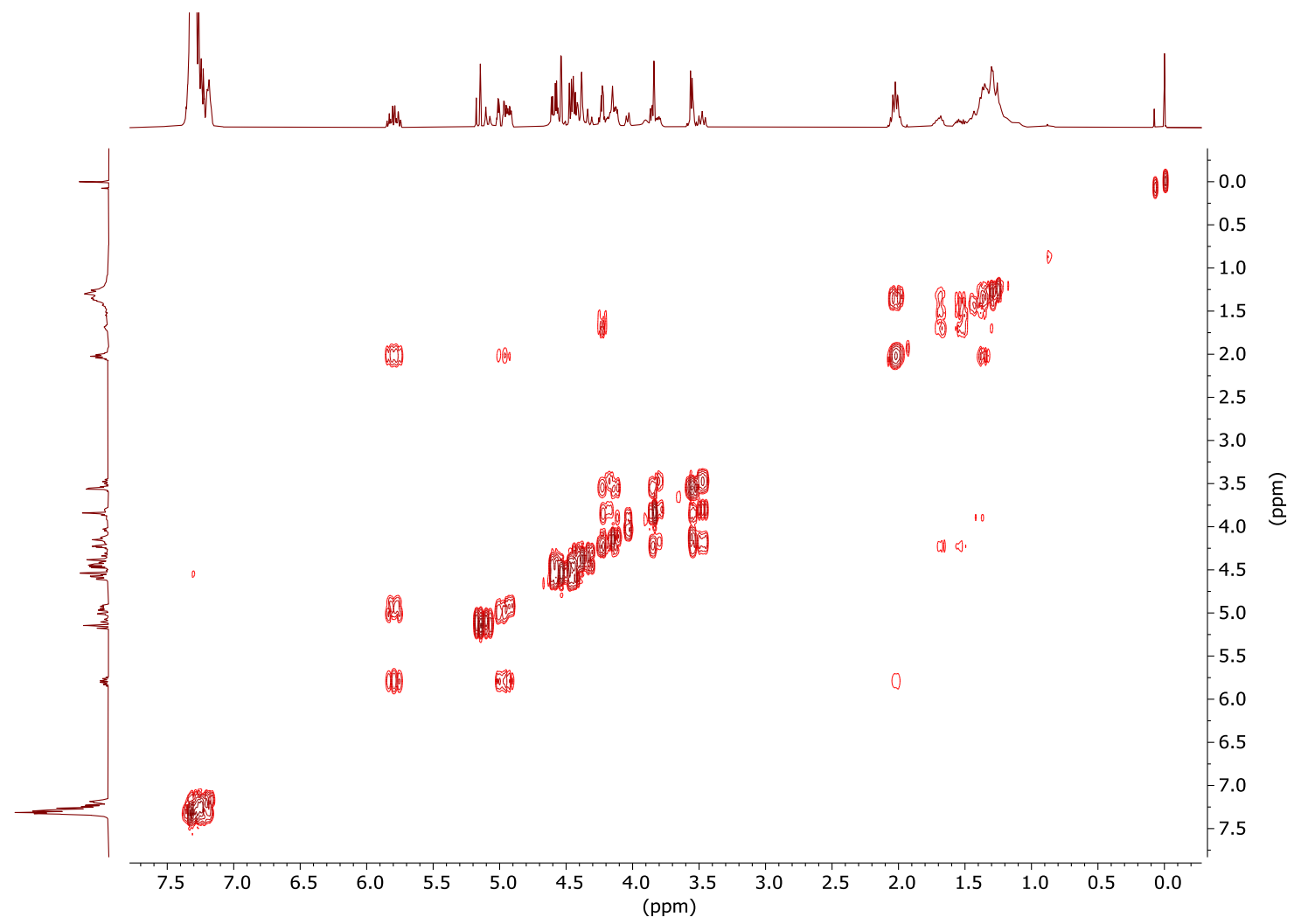

Figure S24. COSY spectrum (400.16 MHz, $\left.\mathrm{CDCl}_{3}\right)$ of $\mathbf{6 b}$ and $\mathbf{1 1 b}$ (1.4:1 mixture).

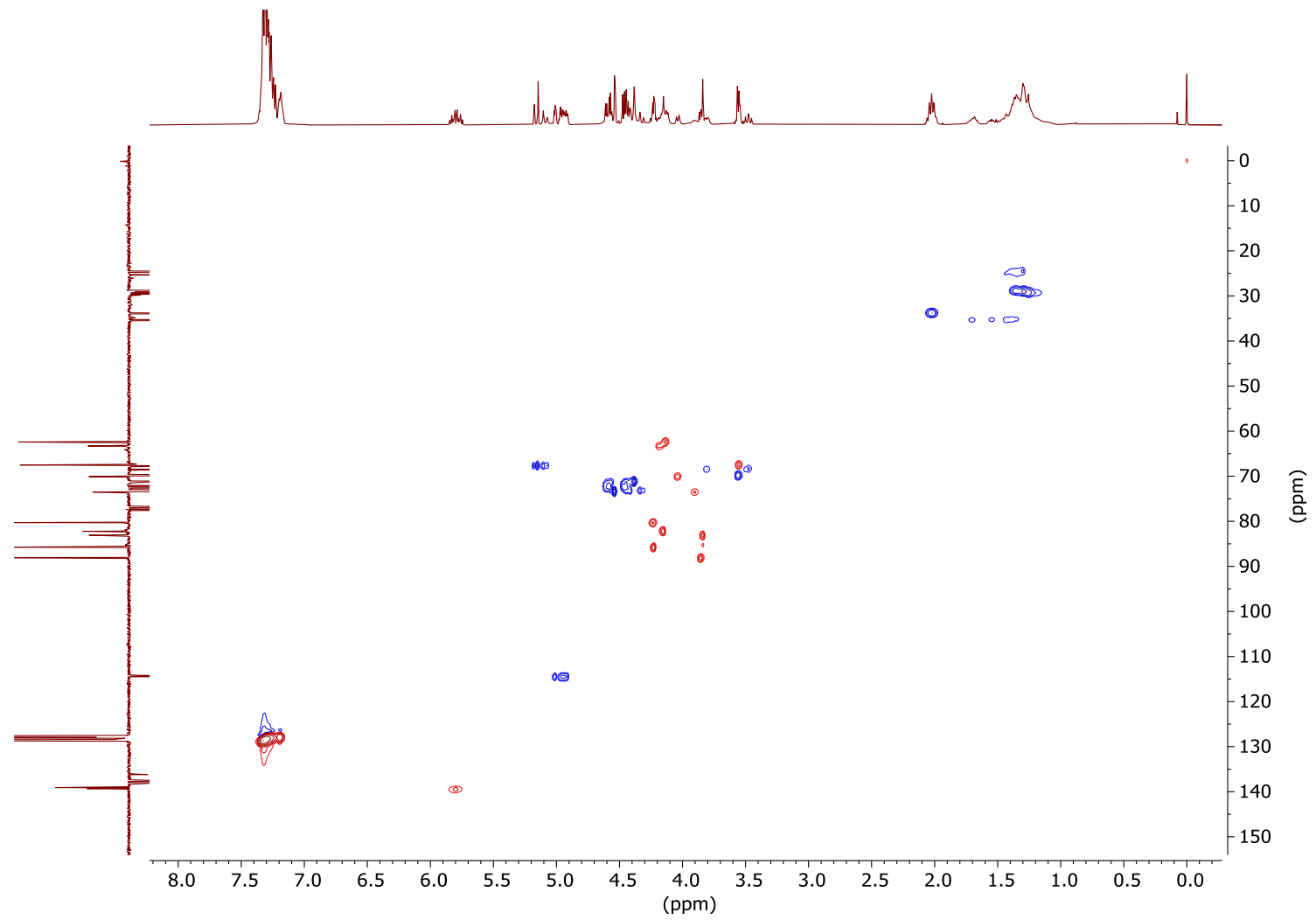

Figure S25. HSQC spectrum (400.16, 100.63 MHz, $\left.\mathrm{CDCl}_{3}\right)$ of $\mathbf{6 b}$ and $\mathbf{1 1 b}(1.4: 1$ mixture). 


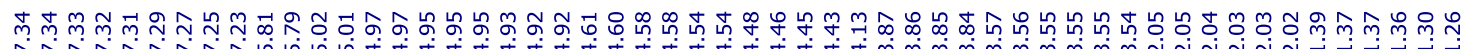

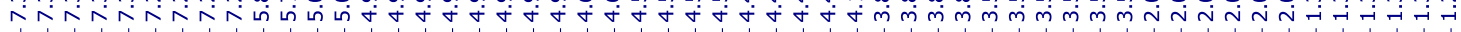
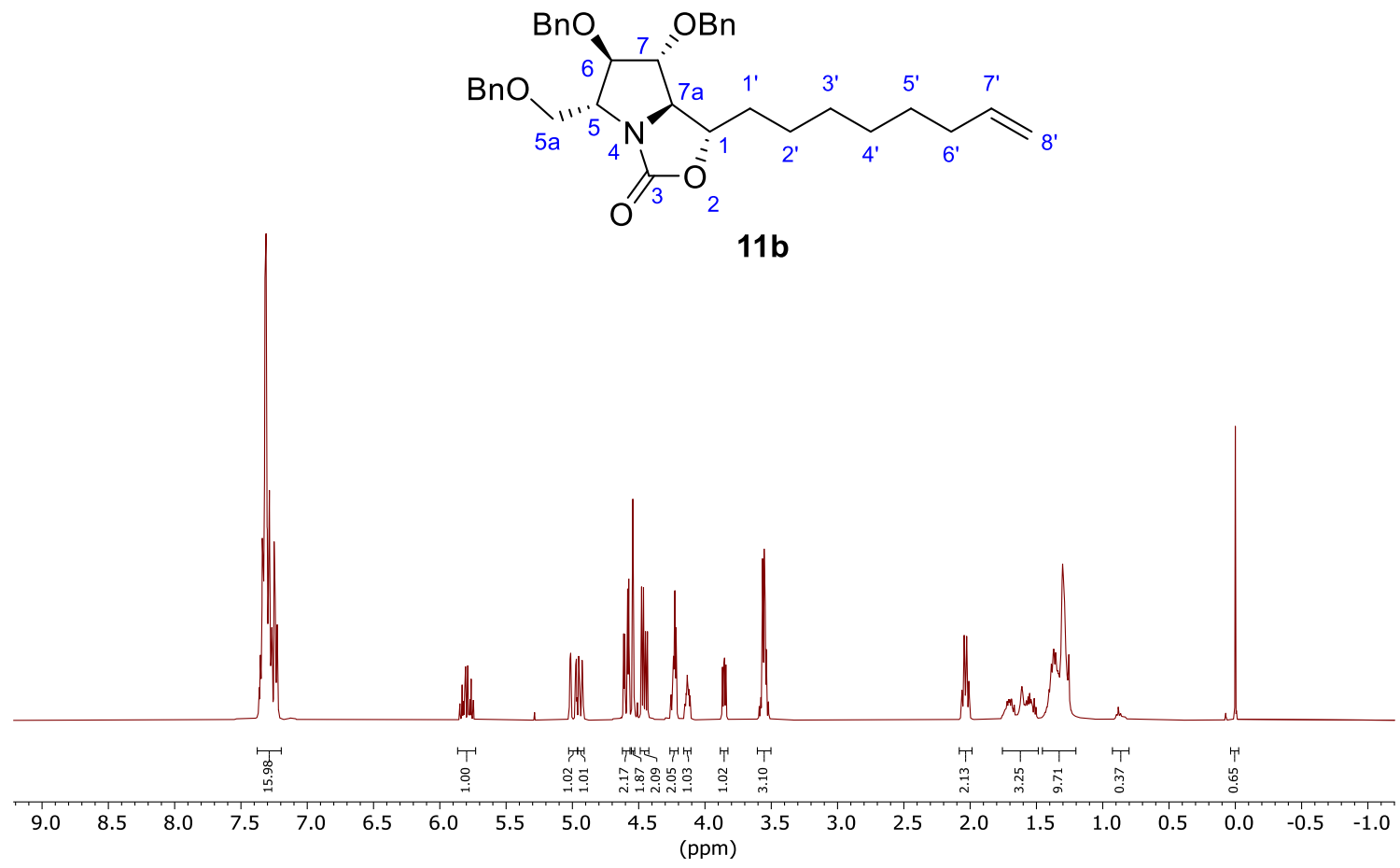

Figure S26. ${ }^{1} \mathrm{H}$ NMR spectrum $\left(400.16 \mathrm{MHz}, \mathrm{CDCl}_{3}\right)$ of $\mathbf{1 1 b}$.
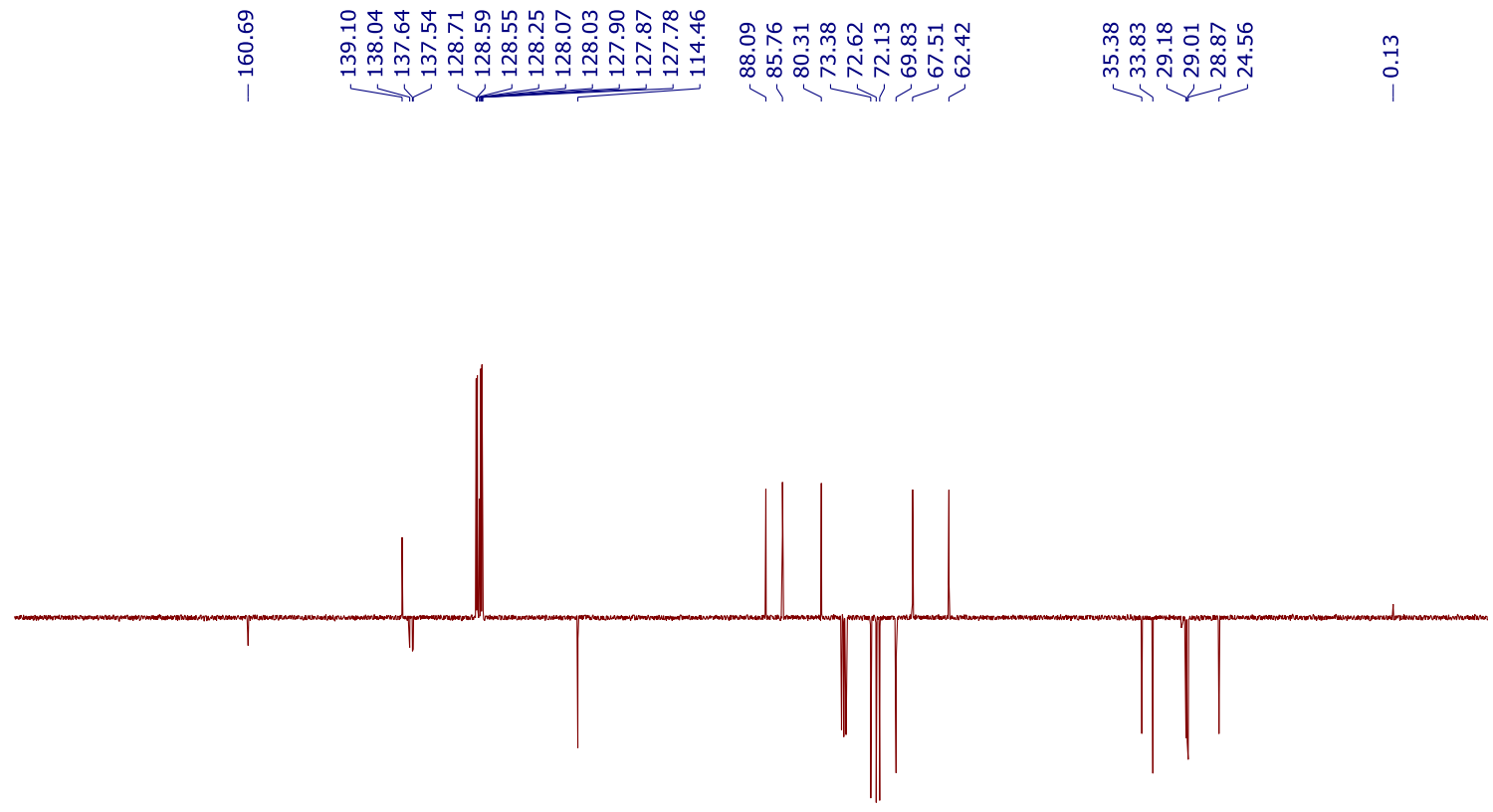

$\begin{array}{lllllllllllllllllllll}190 & 180 & 170 & 160 & 150 & 140 & 130 & 120 & 110 & 100 & 90 & 80 & 70 & 60 & 50 & 40 & 30 & 20 & 10 & 0 & -10\end{array}$

Figure S27. DEPTQ spectrum (100.63 MHz, $\left.\mathrm{CDCl}_{3}\right)$ of $\mathbf{1 1 b}$. 


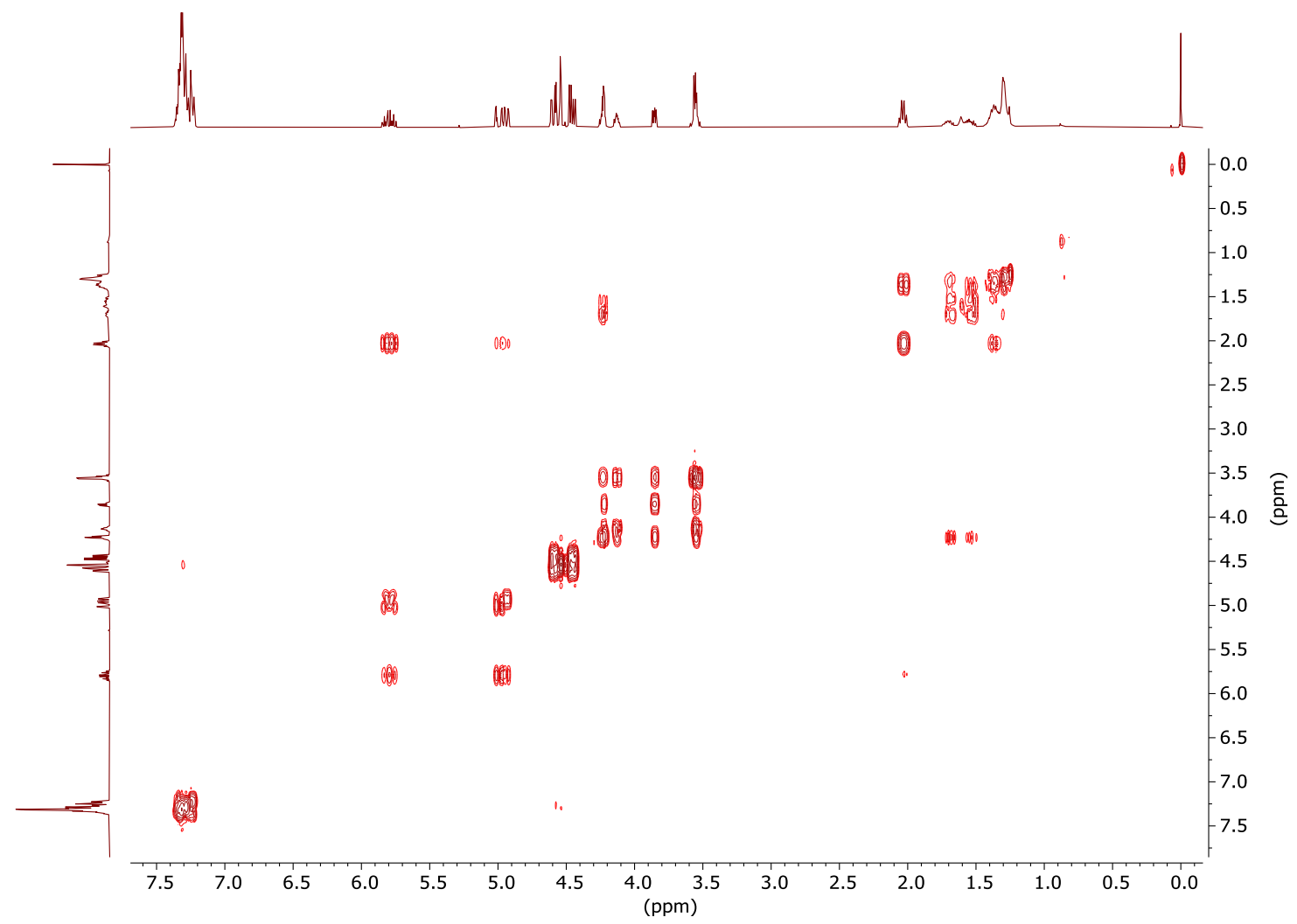

Figure S28. COSY spectrum (400.16 MHz, $\left.\mathrm{CDCl}_{3}\right)$ of $\mathbf{1 1 b}$.

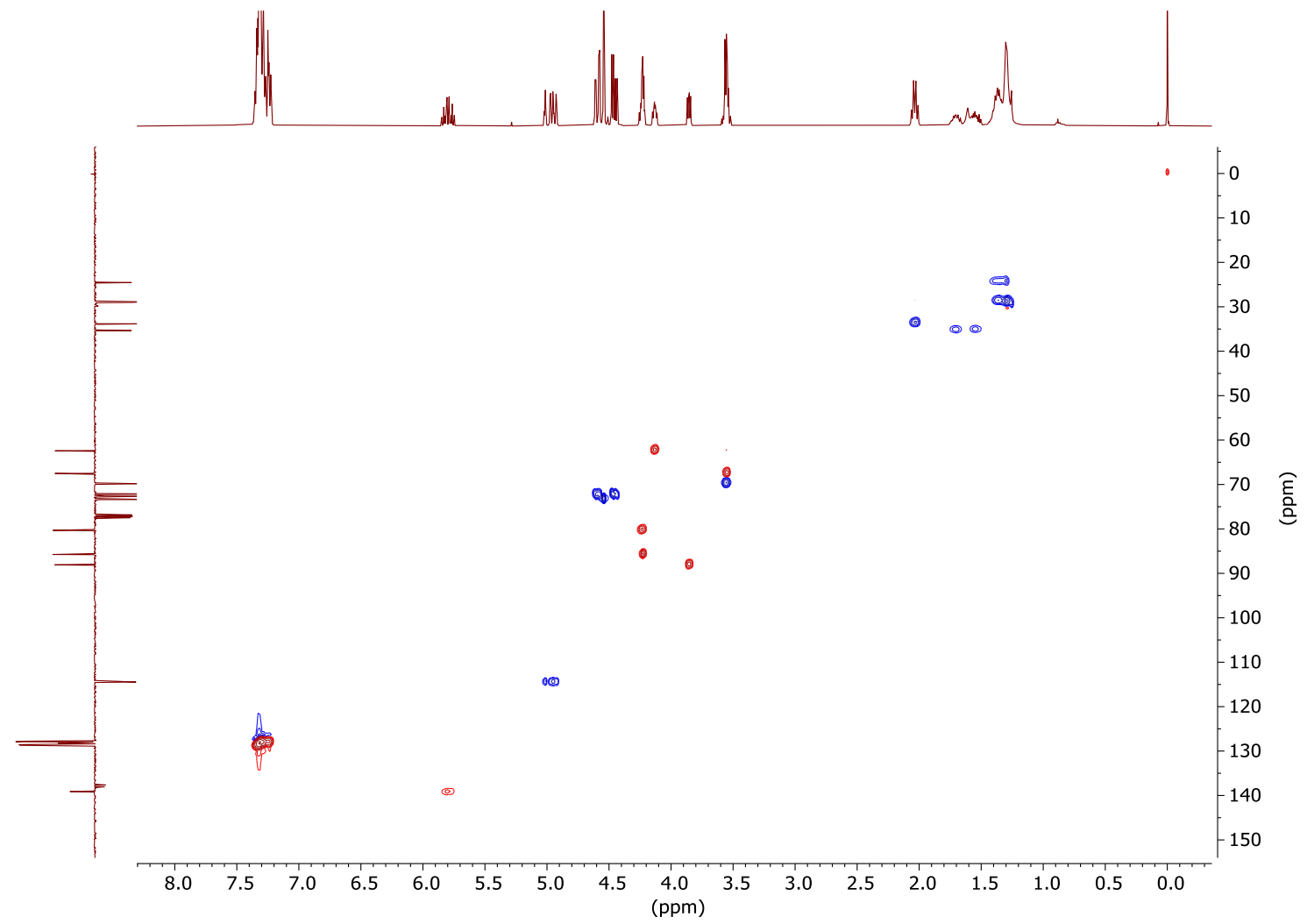

Figure S29. HSQC spectrum $\left(400.16,100.63 \mathrm{MHz}, \mathrm{CDCl}_{3}\right)$ of $\mathbf{1 1 b}$. 


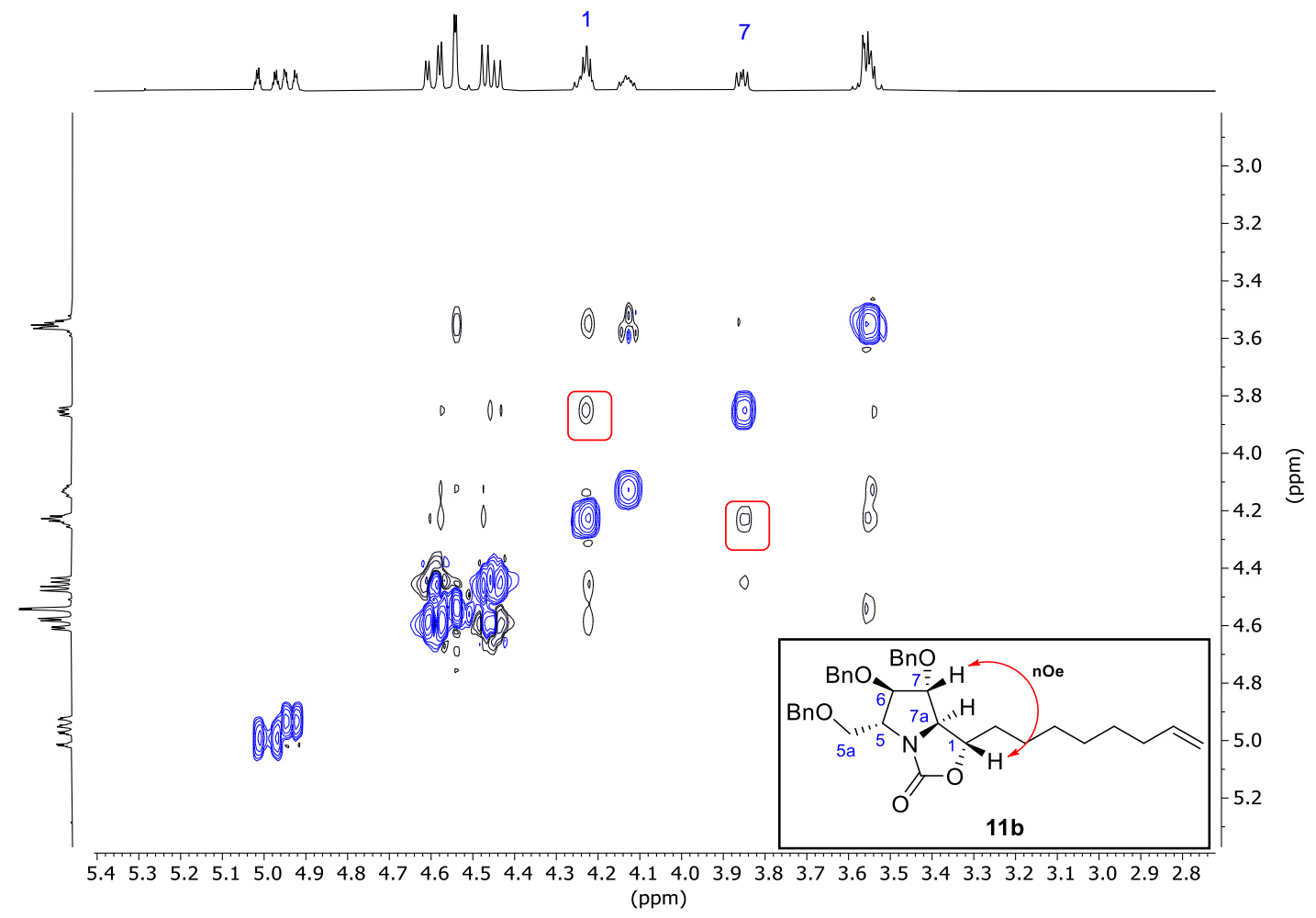

Figure S30. 2D NOESY spectrum $\left(400.16 \mathrm{MHz}, \mathrm{CDCl}_{3}\right)$ of $\mathbf{1 1 b}$.
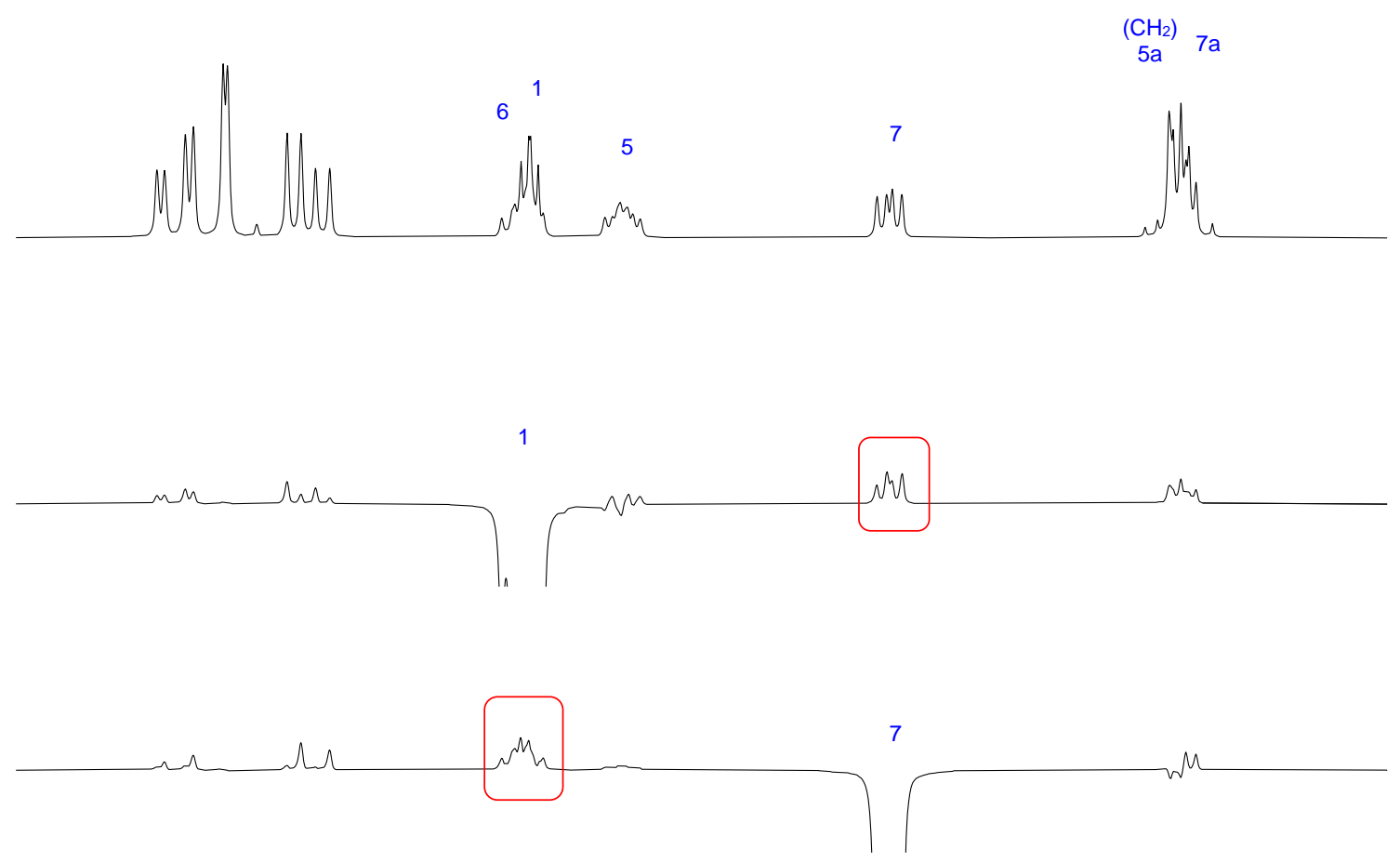

\begin{tabular}{|c|c|c|c|c|c|c|c|c|c|c|c|c|c|}
\hline 4.7 & 4.6 & 4.5 & 4.4 & 4.3 & 4.2 & 4.1 & 4.0 & 3.9 & 3.8 & 3.7 & 3.6 & 3.5 & 3.4 \\
\hline
\end{tabular}

Figure S31. ${ }^{1} \mathrm{H}$ NMR and 1D NOESY spectra $\left(400.16 \mathrm{MHz}, \mathrm{CDCl}_{3}\right)$ of $\mathbf{1 1 b .}$ 


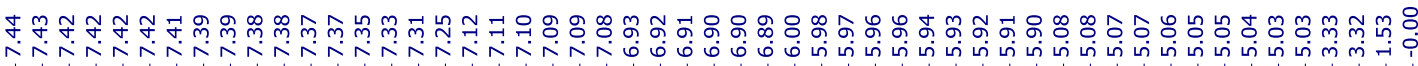

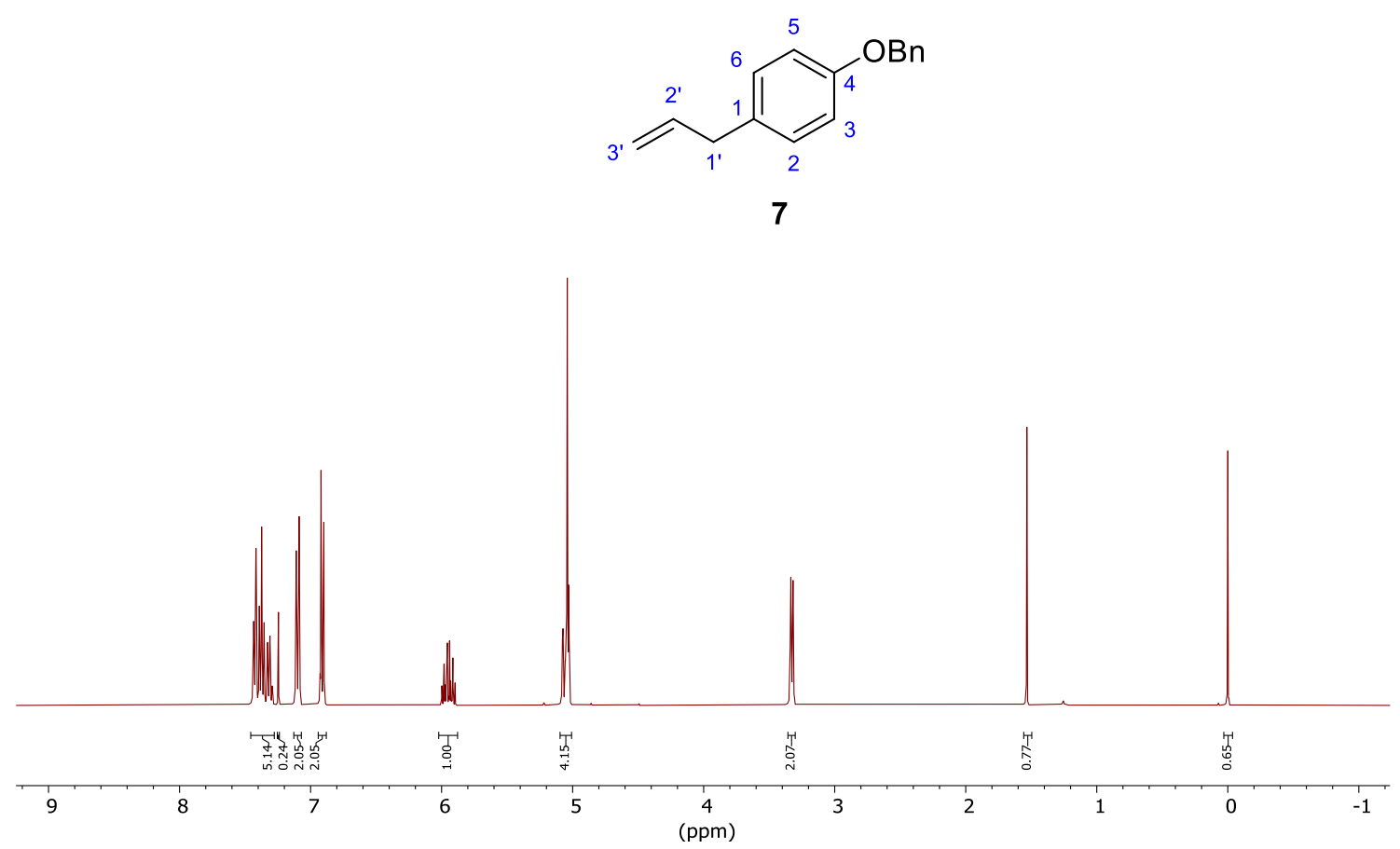

Figure S32. ${ }^{1} \mathrm{H}$ NMR spectrum $\left(400.16 \mathrm{MHz}, \mathrm{CDCl}_{3}\right)$ of 7.
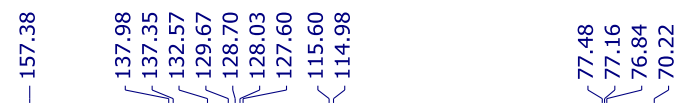

ㅇํำ
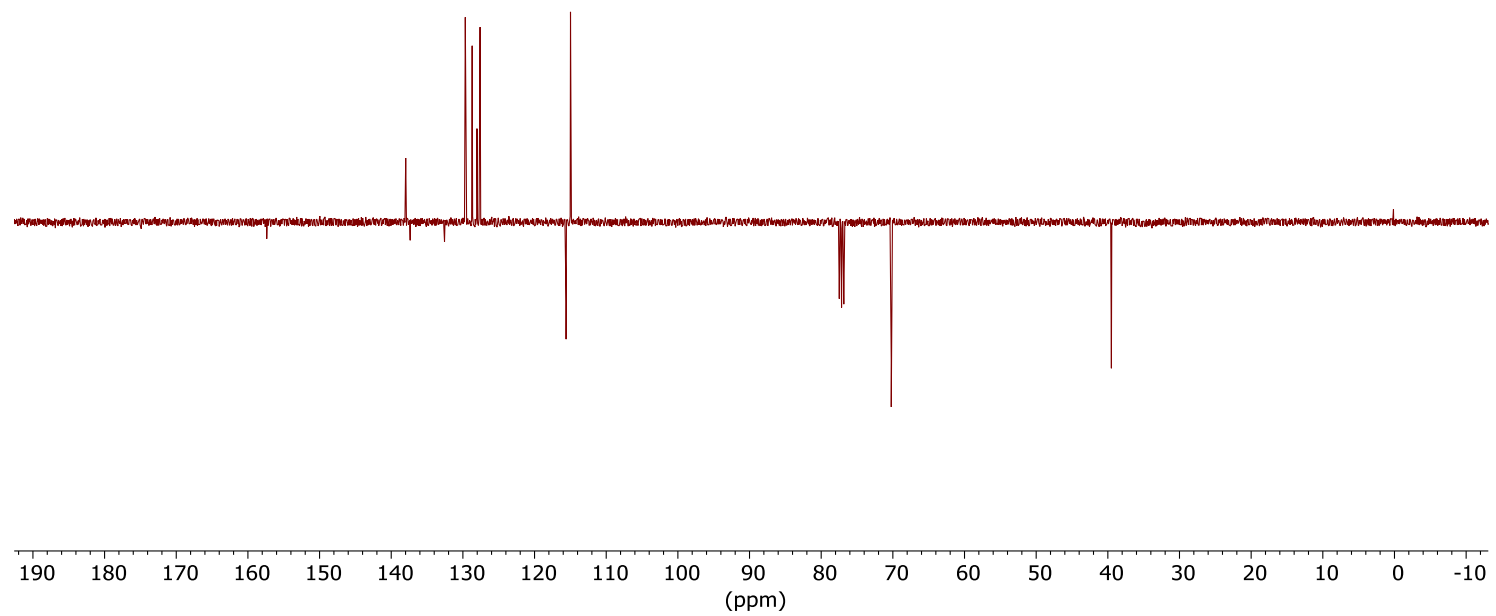

Figure S33. DEPTQ spectrum $\left(100.63 \mathrm{MHz}, \mathrm{CDCl}_{3}\right)$ of 7. 


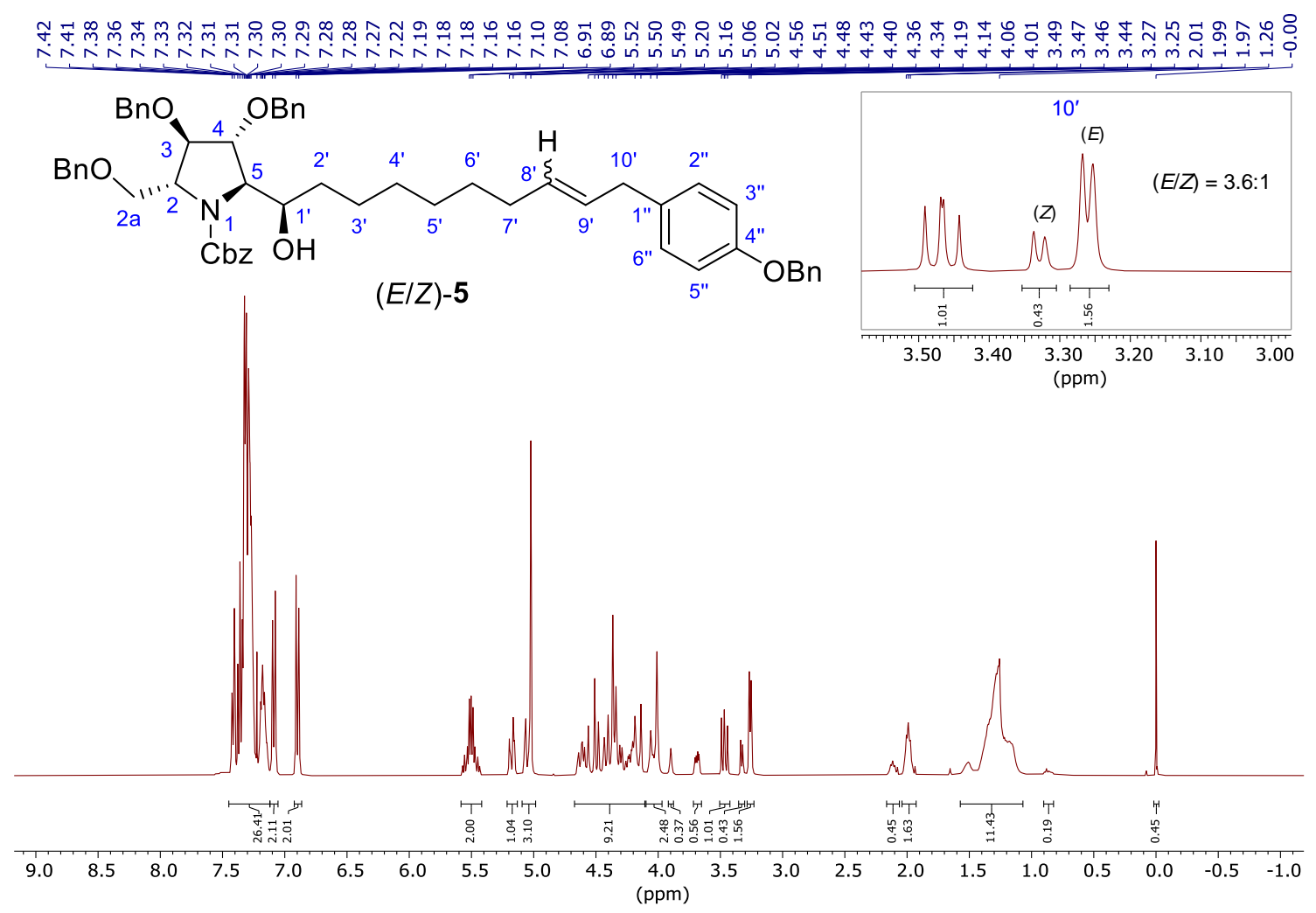

Figure S34. ${ }^{1} \mathrm{H}$ NMR spectrum $\left(400.16 \mathrm{MHz}, \mathrm{CDCl}_{3} / \mathrm{D}_{2} \mathrm{O}\right)$ of $(E / Z)-\mathbf{5}$ (1.5:1 mixture of rotamers).

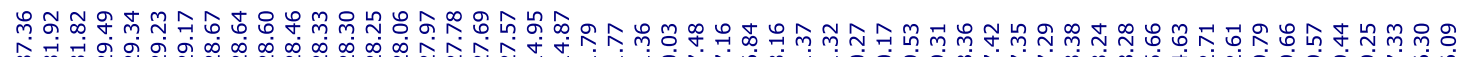

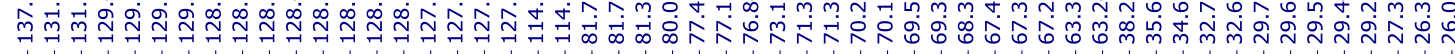

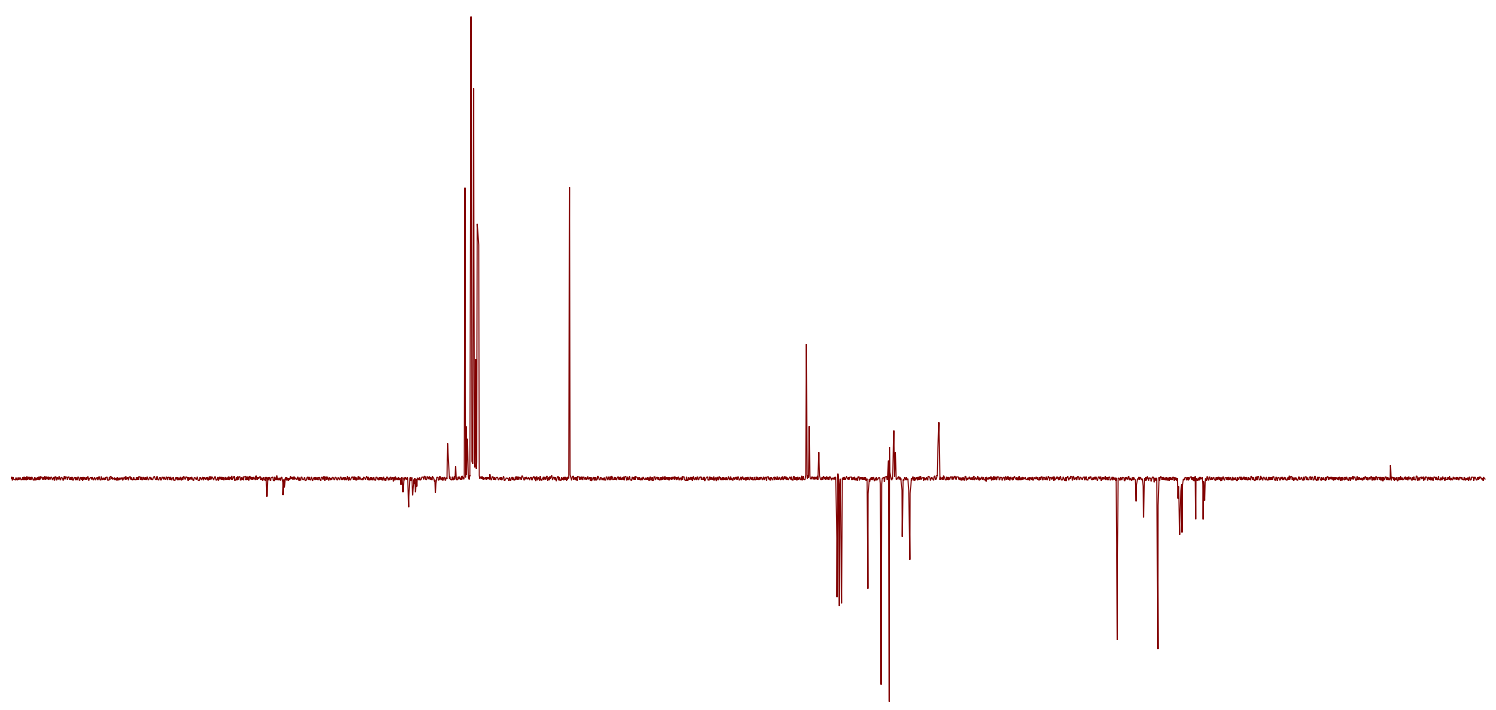

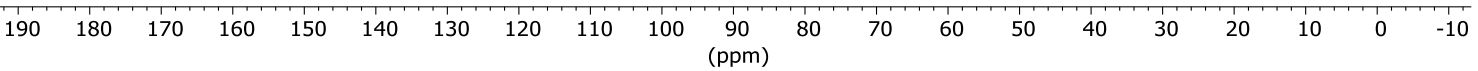

Figure S35. DEPTQ spectrum (100.63 MHz, $\left.\mathrm{CDCl}_{3} / \mathrm{D}_{2} \mathrm{O}\right)$ of $(E / Z)-5$ (1.5:1 mixture of rotamers). 


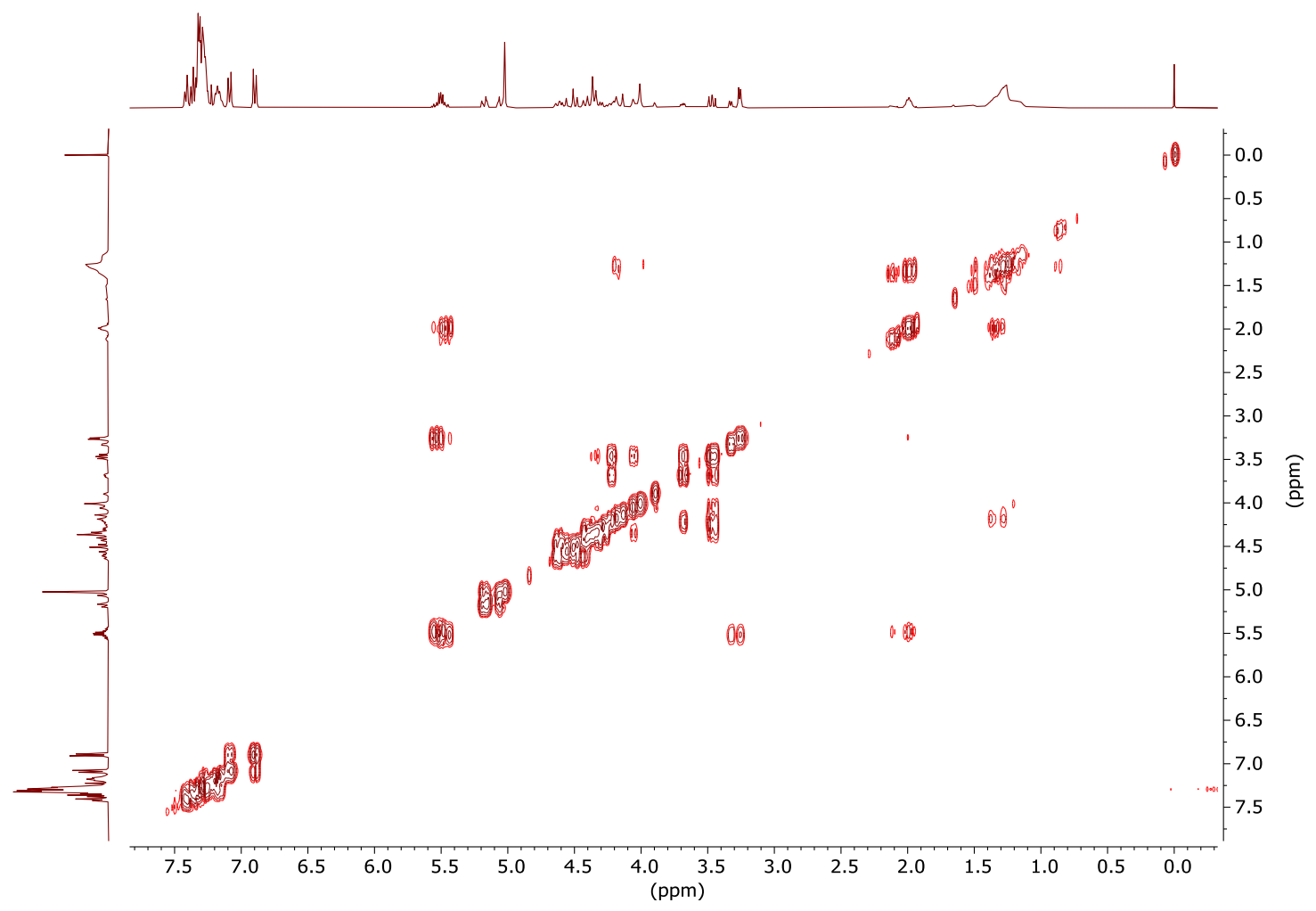

Figure S36. COSY spectrum (400.16 MHz, $\left.\mathrm{CDCl}_{3} / \mathrm{D}_{2} \mathrm{O}\right)$ of $(E / Z)-5$.

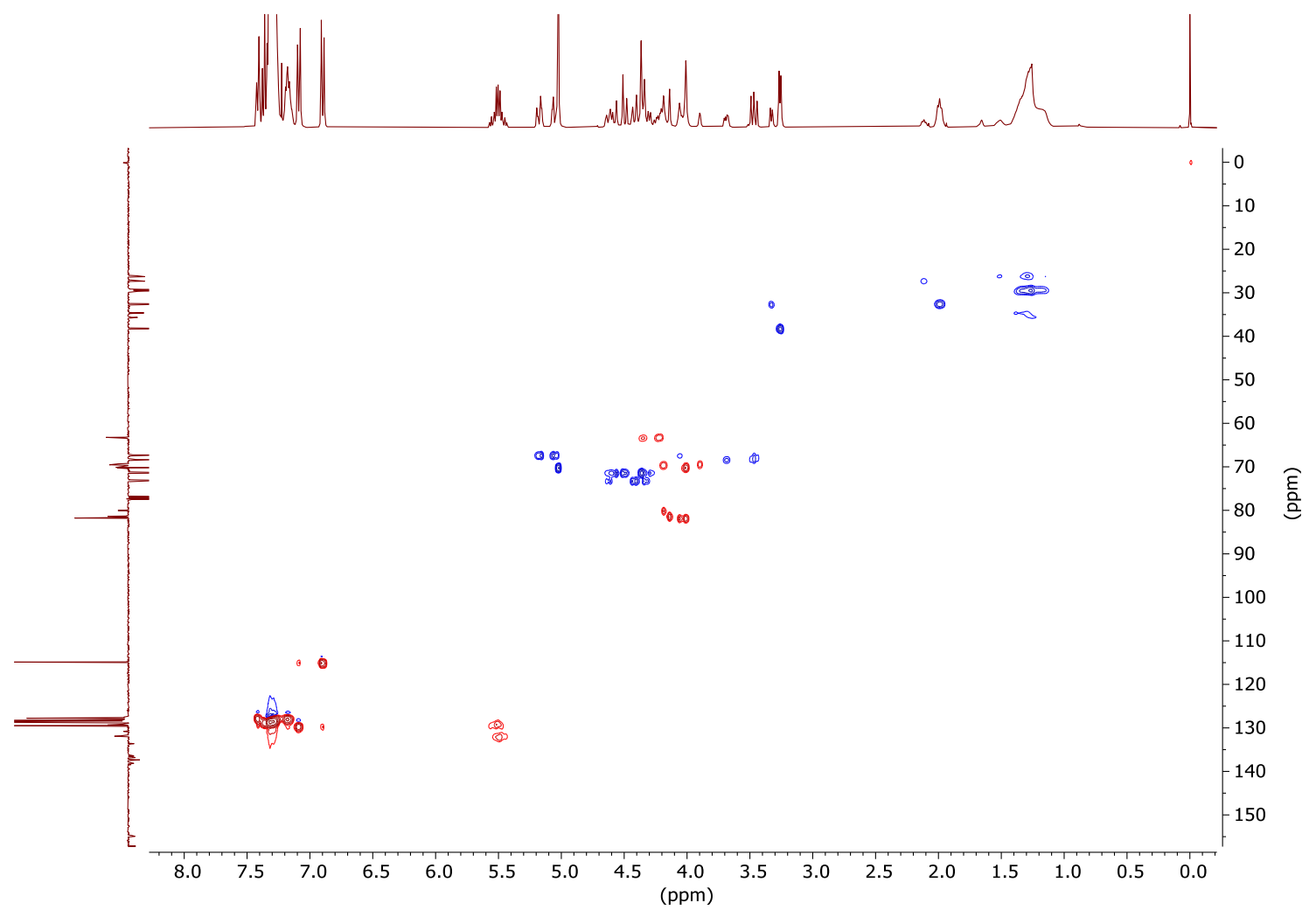

Figure S37. HSQC spectrum (400.16, $\left.100.63 \mathrm{MHz}, \mathrm{CDCl}_{3} / \mathrm{D}_{2} \mathrm{O}\right)$ of $(E / Z)-5$. 

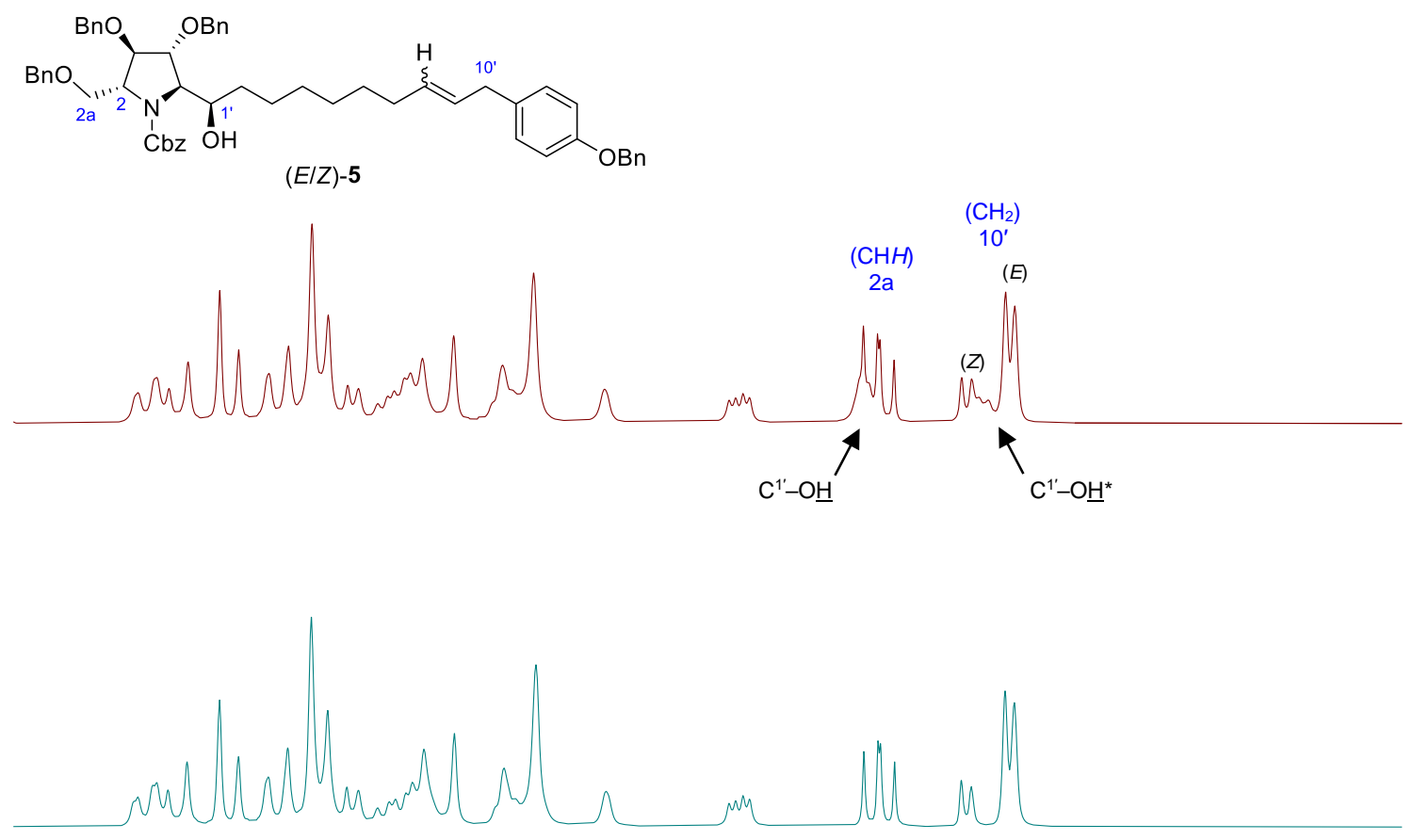

\begin{tabular}{llllllllllllllllllllll}
\hline 4.8 & 4.7 & 4.6 & 4.5 & 4.4 & 4.3 & 4.2 & 4.1 & 4.0 & 3.9 & $\begin{array}{c}3.8 \\
(\mathrm{ppm})\end{array}$ & 3.7 & 3.6 & 3.5 & 3.4 & 3.3 & 3.2 & 3.1 & 3.0 & 2.9 & 2.8 & 2.7
\end{tabular}

Figure S38. ${ }^{1} \mathrm{H}$ NMR spectra (400.16 MHz) of (E/Z)-5 in (a) $\mathrm{CDCl}_{3}$ (maroon) and (b) $\mathrm{CDCl}_{3} / \mathrm{D}_{2} \mathrm{O}$ (teal).

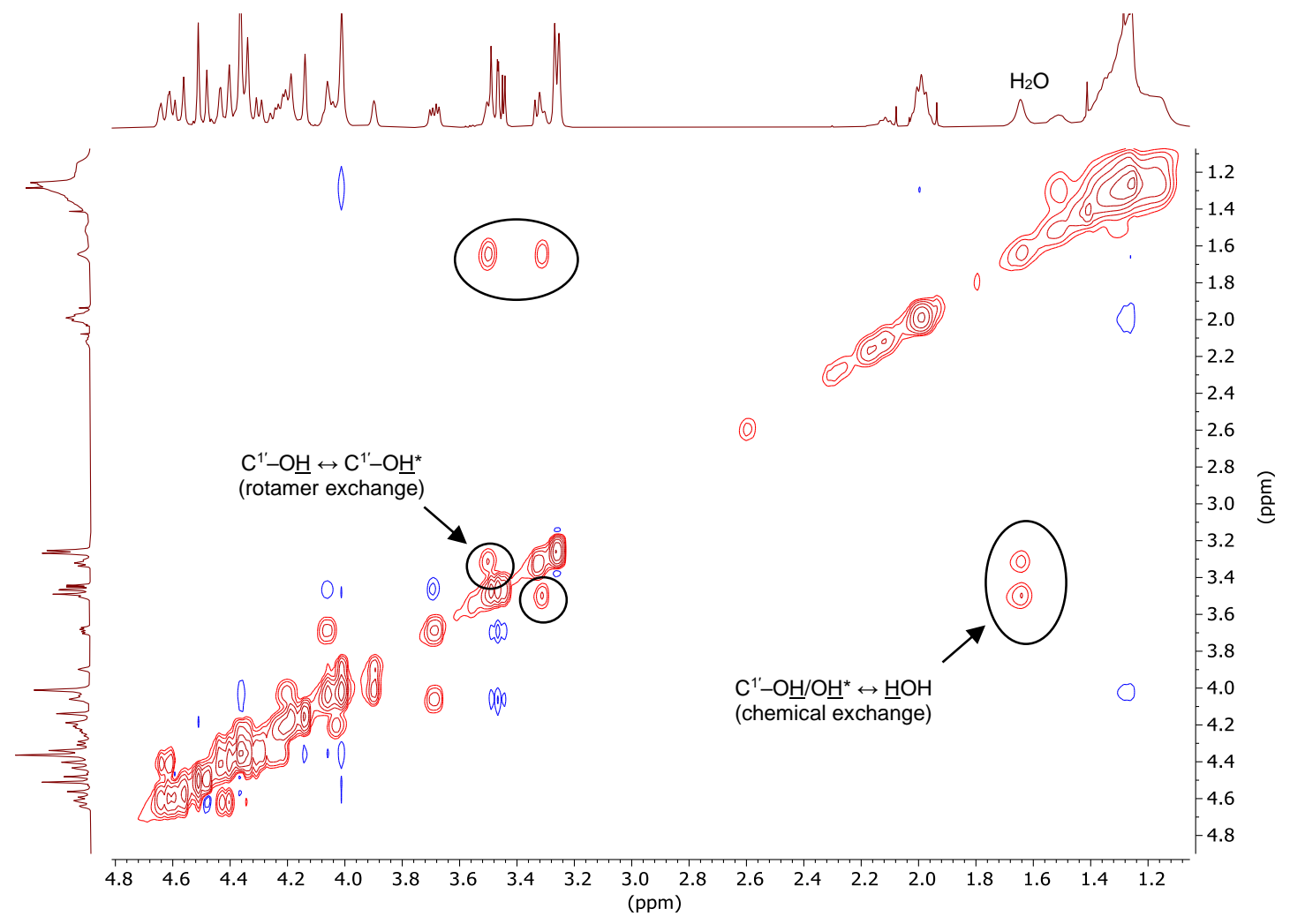

Figure S39. 2D NOESY spectrum (400.16 MHz, $\left.\mathrm{CDCl}_{3}\right)$ of $(E / Z)-5$. 

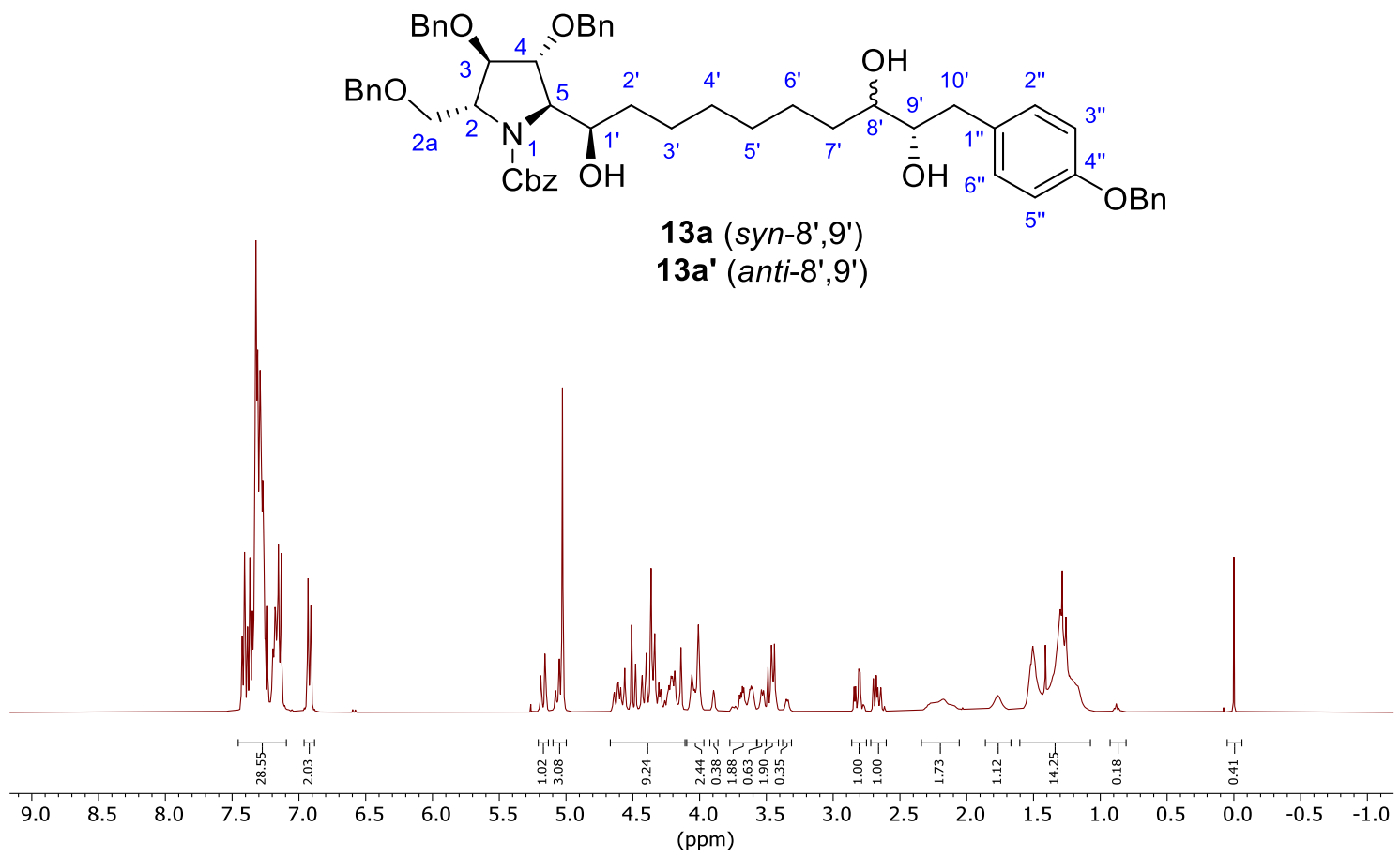

Figure S40. ${ }^{1} \mathrm{H}$ NMR spectrum $\left(400.16 \mathrm{MHz}, \mathrm{CDCl}_{3}\right)$ of $\mathbf{1 3 a} / \mathbf{1 3 a} \mathbf{a}^{\prime}$ (1.7:1 mixture of rotamers).

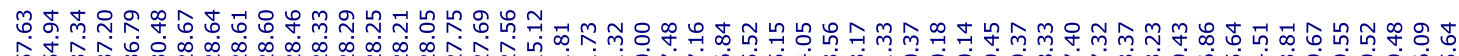

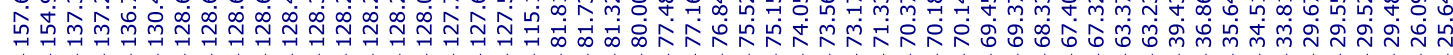

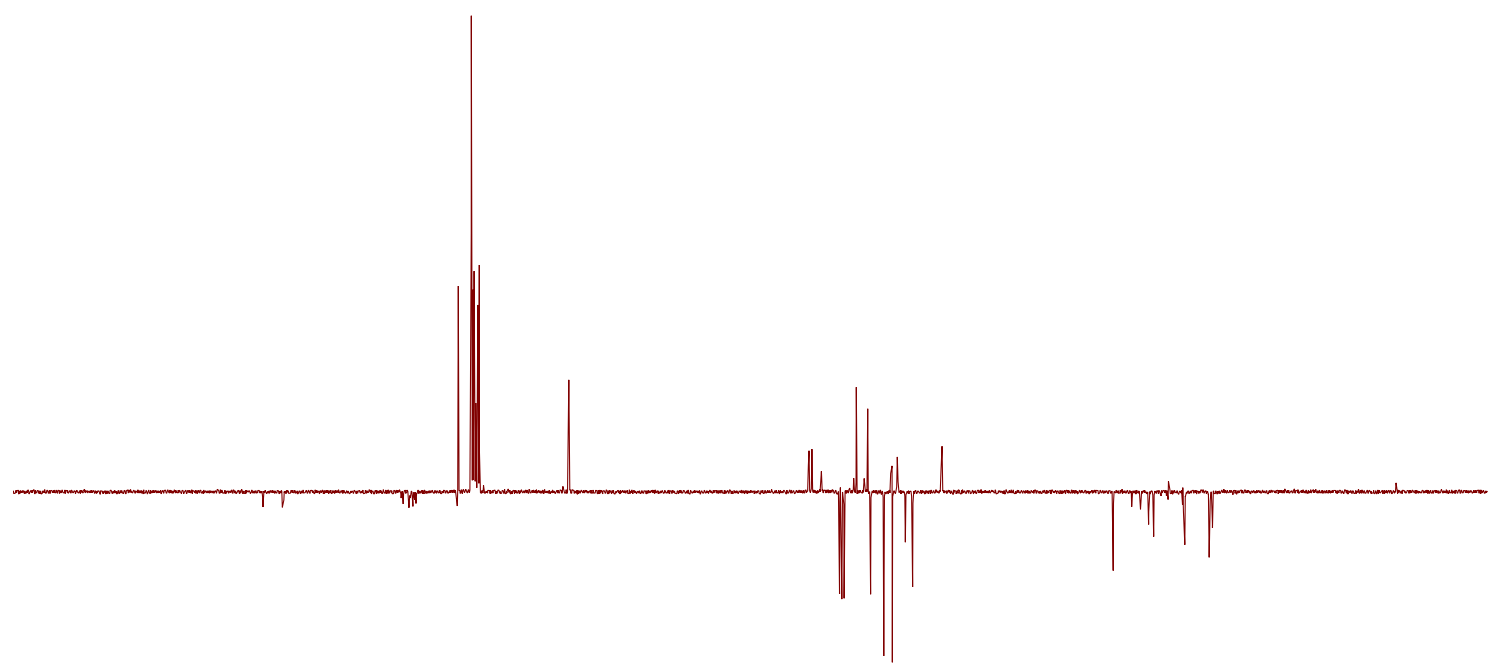

$\begin{array}{lllllllllllllllllllll}190 & 180 & 170 & 160 & 150 & 140 & 130 & 120 & 110 & 100 & \begin{array}{c}90 \\ (\mathrm{ppm})\end{array} & 80 & 70 & 60 & 50 & 40 & 30 & 20 & 10 & 0 & -10\end{array}$

Figure S41. DEPTQ spectrum (100.63 MHz, $\left.\mathrm{CDCl}_{3}\right)$ of 13a/13a' (1.7:1 mixture of rotamers). 


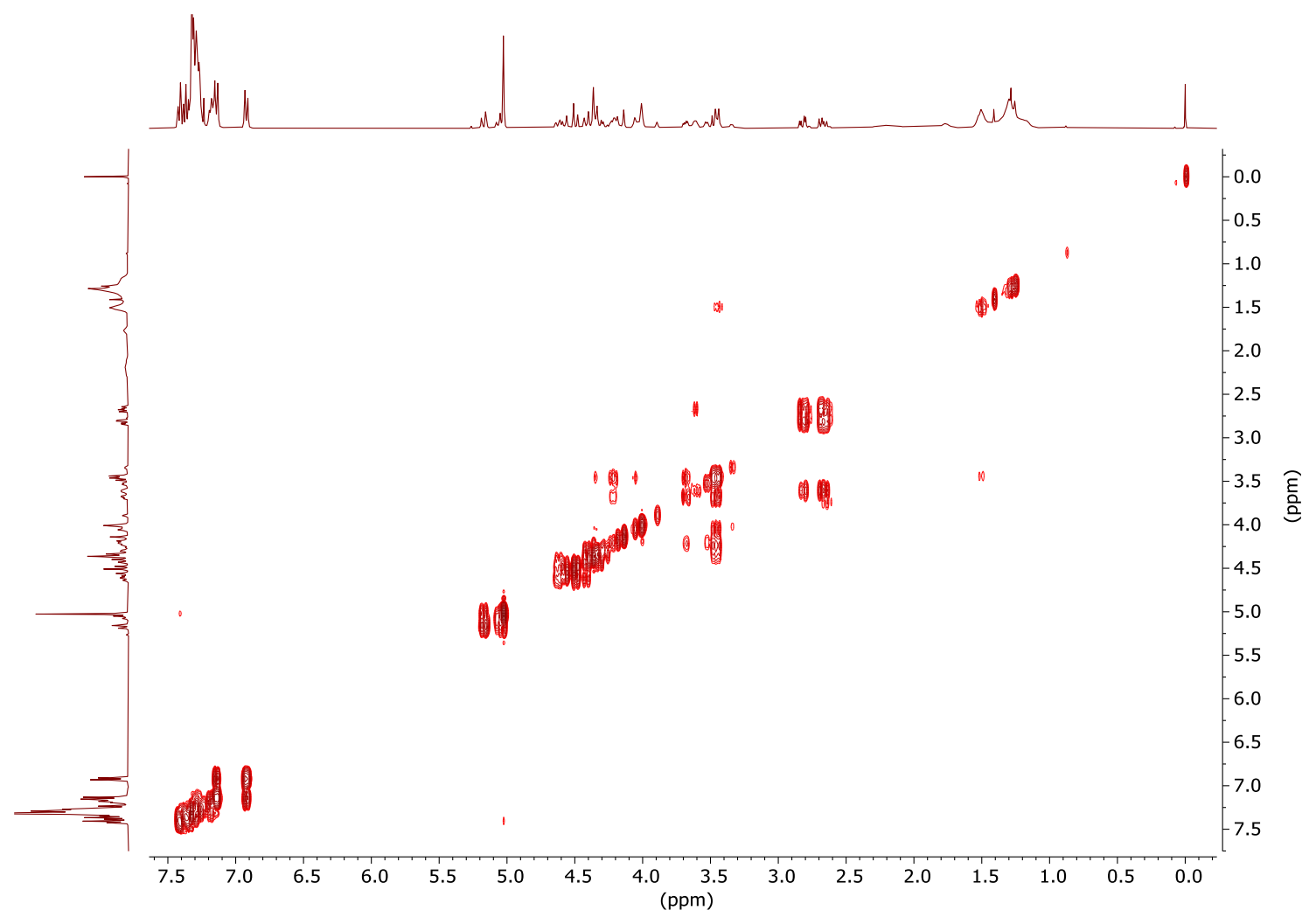

Figure S42. COSY spectrum $\left(400.16 \mathrm{MHz}, \mathrm{CDCl}_{3}\right)$ of $\mathbf{1 3 a} / \mathbf{1 3 a}^{\prime}$.

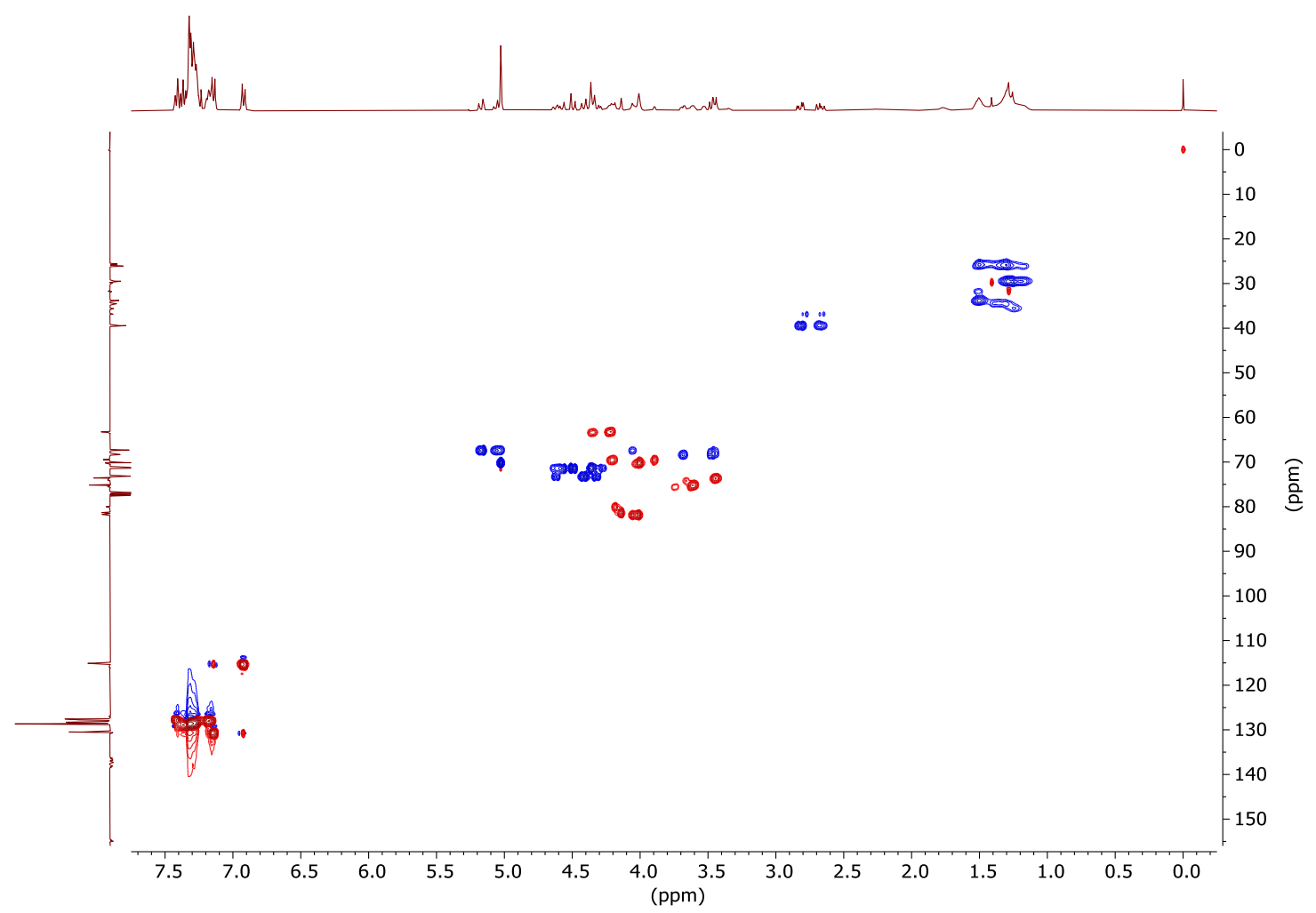

Figure S43. HSQC spectrum $\left(400.16,100.63 \mathrm{MHz}, \mathrm{CDCl}_{3}\right)$ of 13a/13a'. 


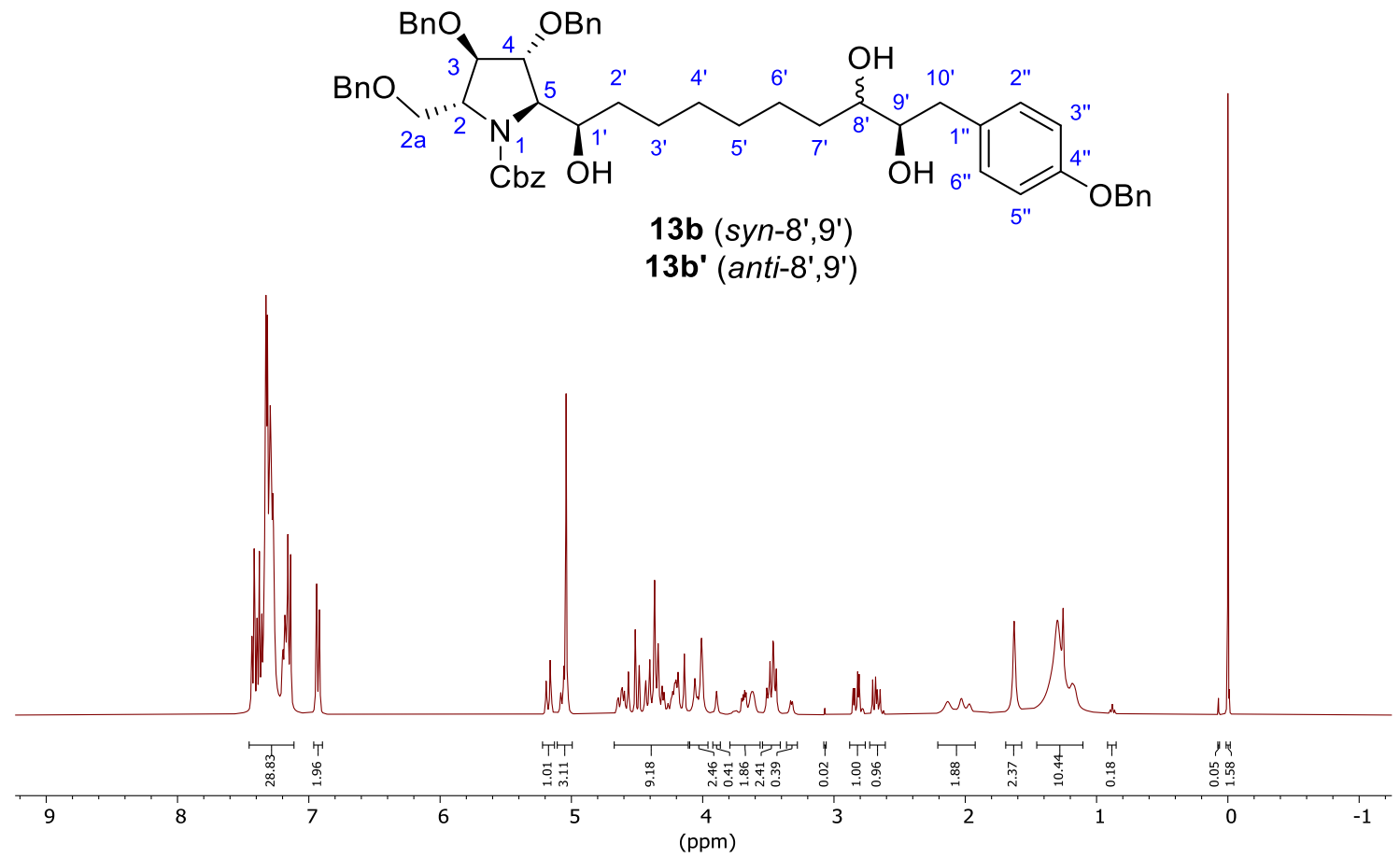

Figure S44. ${ }^{1} \mathrm{H}$ NMR spectrum (400.16 MHz, $\left.\mathrm{CDCl}_{3}\right)$ of $\mathbf{1 3 b} / \mathbf{1 3 b}$ ' (1.7:1 mixture of rotamers).

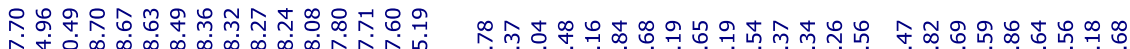
ĥન
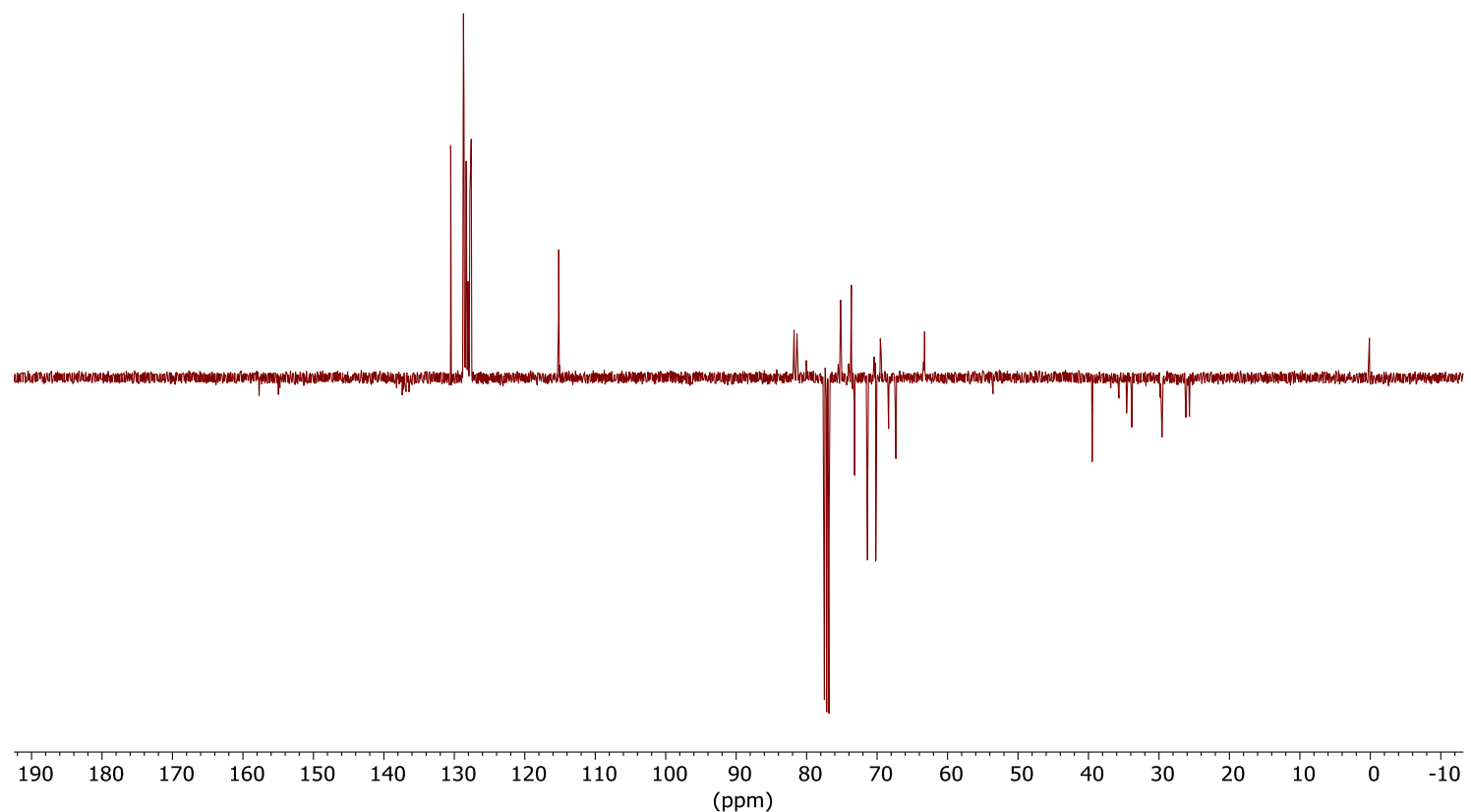

Figure S45. DEPTQ spectrum (100.63 MHz, $\left.\mathrm{CDCl}_{3}\right)$ of $\mathbf{1 3 b} / \mathbf{1 3} \mathbf{b}^{\prime}$ (1.7:1 mixture of rotamers). 


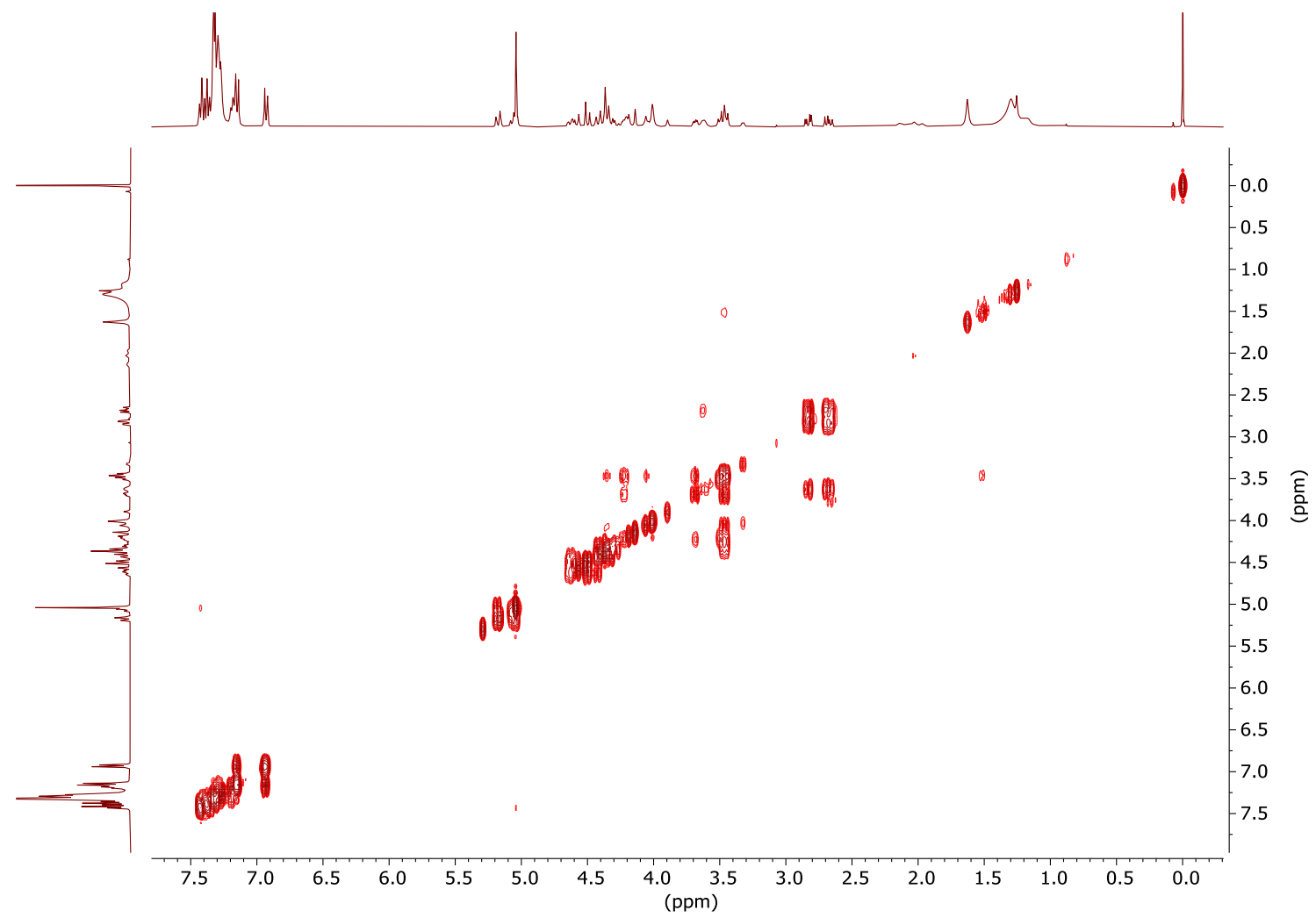

Figure S46. COSY spectrum (400.16 MHz, $\left.\mathrm{CDCl}_{3}\right)$ of $\mathbf{1 3 b} / \mathbf{1 3 b}$ '.

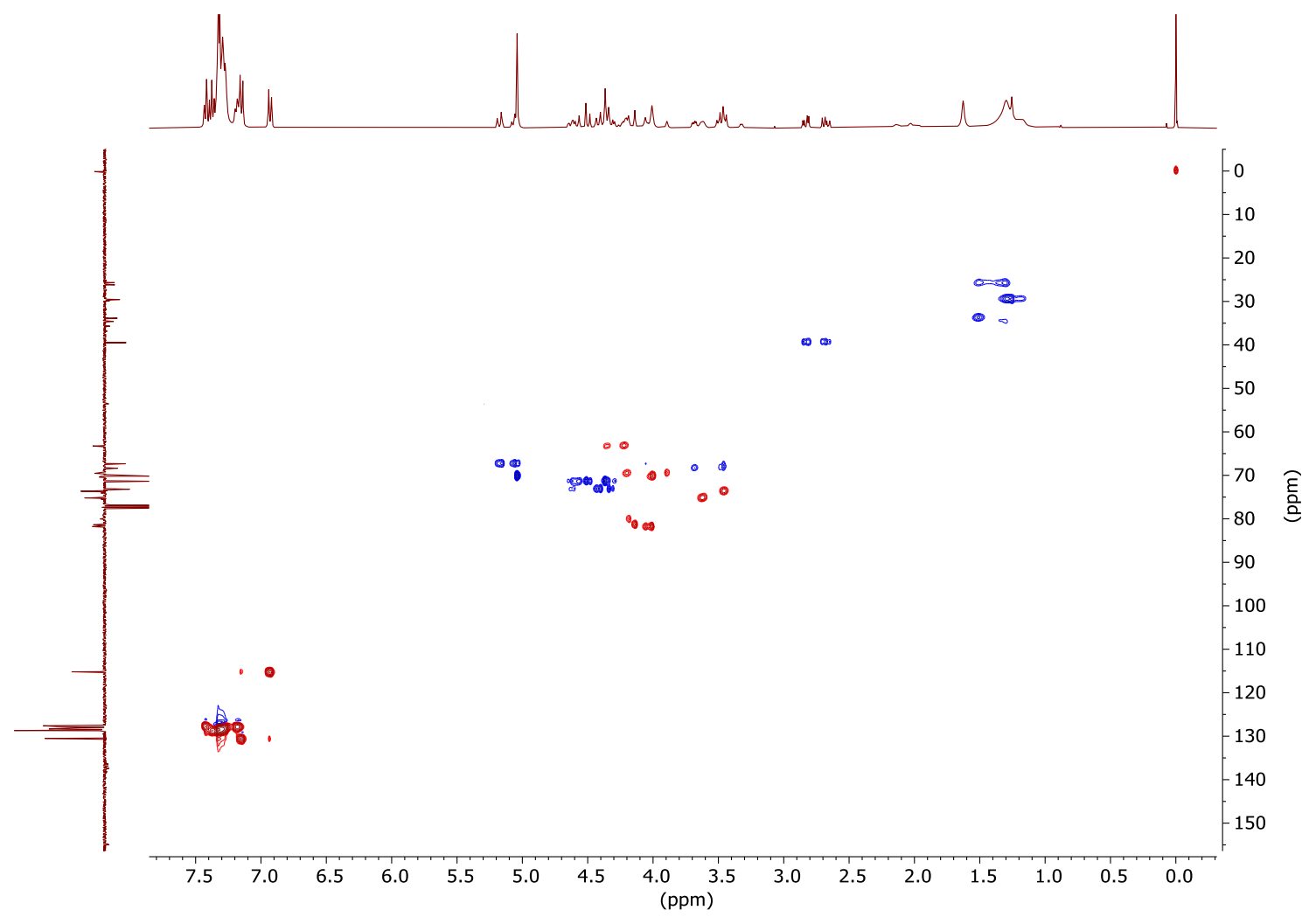

Figure S47. HSQC spectrum $\left(400.16,100.63 \mathrm{MHz}, \mathrm{CDCl}_{3}\right)$ of $\mathbf{1 3 b} / \mathbf{1 3} \mathbf{b}^{\prime}$. 


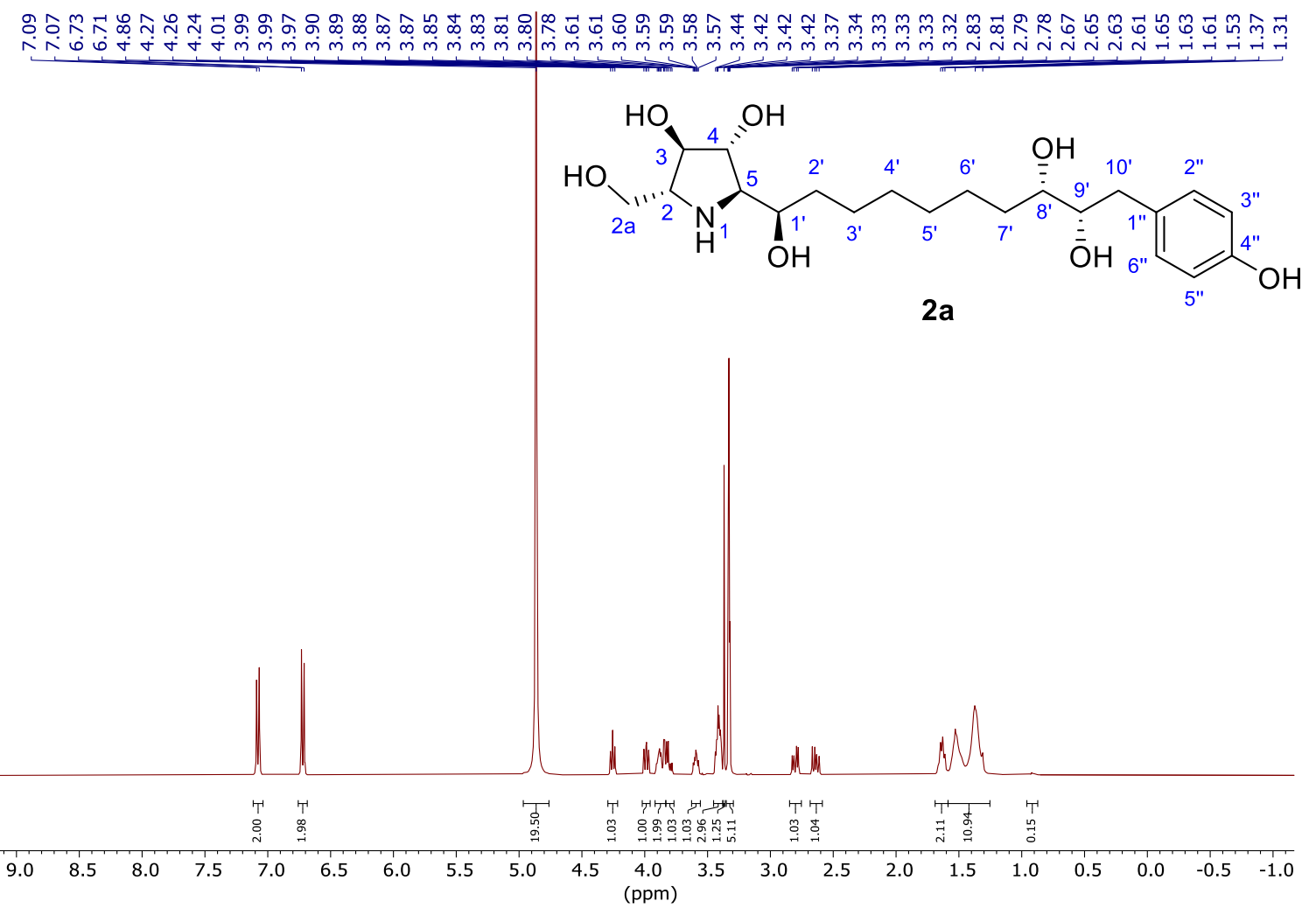

Figure S48. ${ }^{1} \mathrm{H}$ NMR spectrum $\left(400.16 \mathrm{MHz}, \mathrm{CD}_{3} \mathrm{OD}\right)$ of $\mathbf{2 a} \cdot \mathrm{TFA}$.
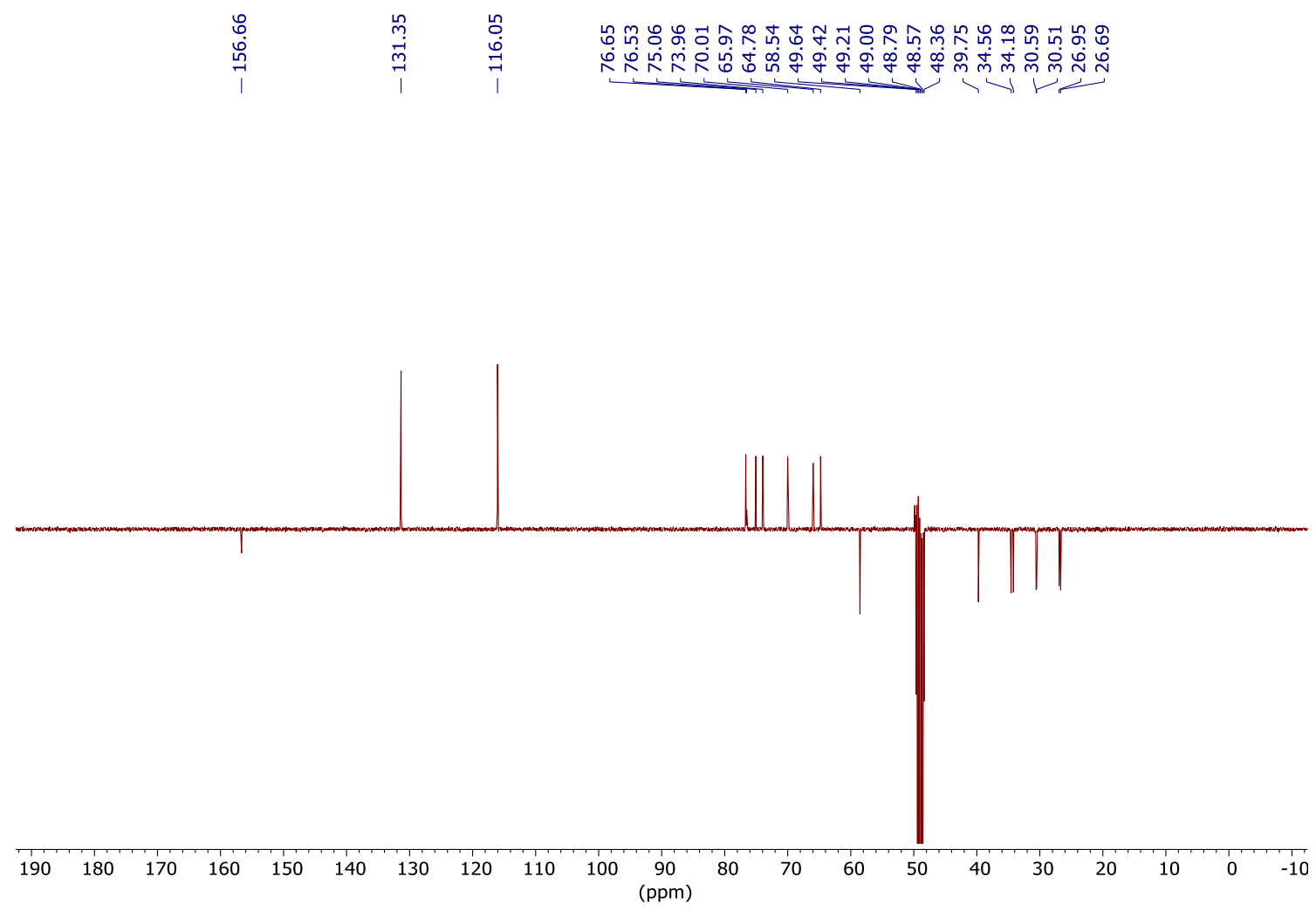

Figure S49. DEPTQ spectrum (100.67 MHz, $\left.\mathrm{CD}_{3} \mathrm{OD}\right)$ of 2a.TFA. 


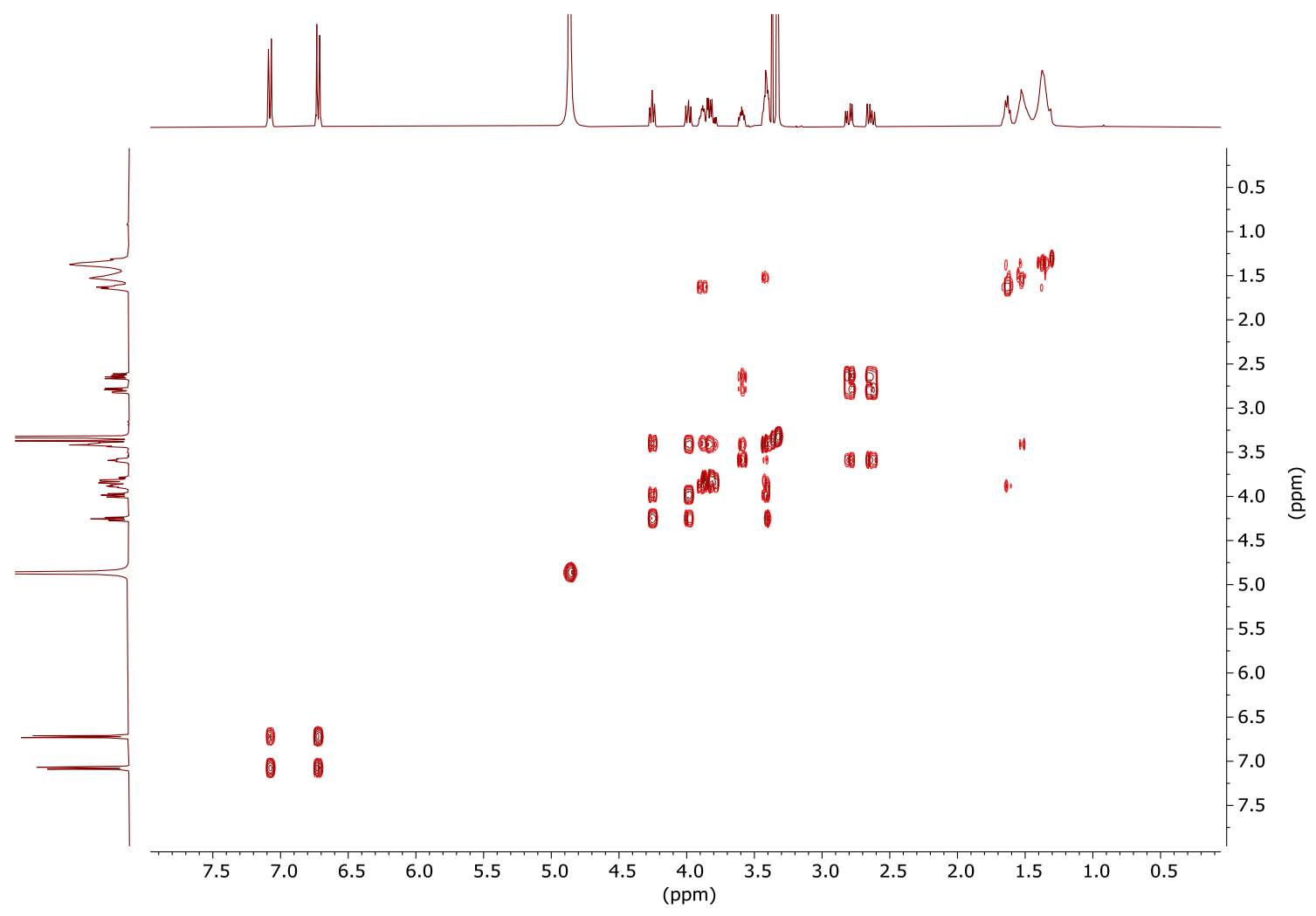

Figure S50. COSY spectrum (400.16 MHz, $\left.\mathrm{CD}_{3} \mathrm{OD}\right)$ of $\mathbf{2 a} \cdot \mathrm{TFA}$.

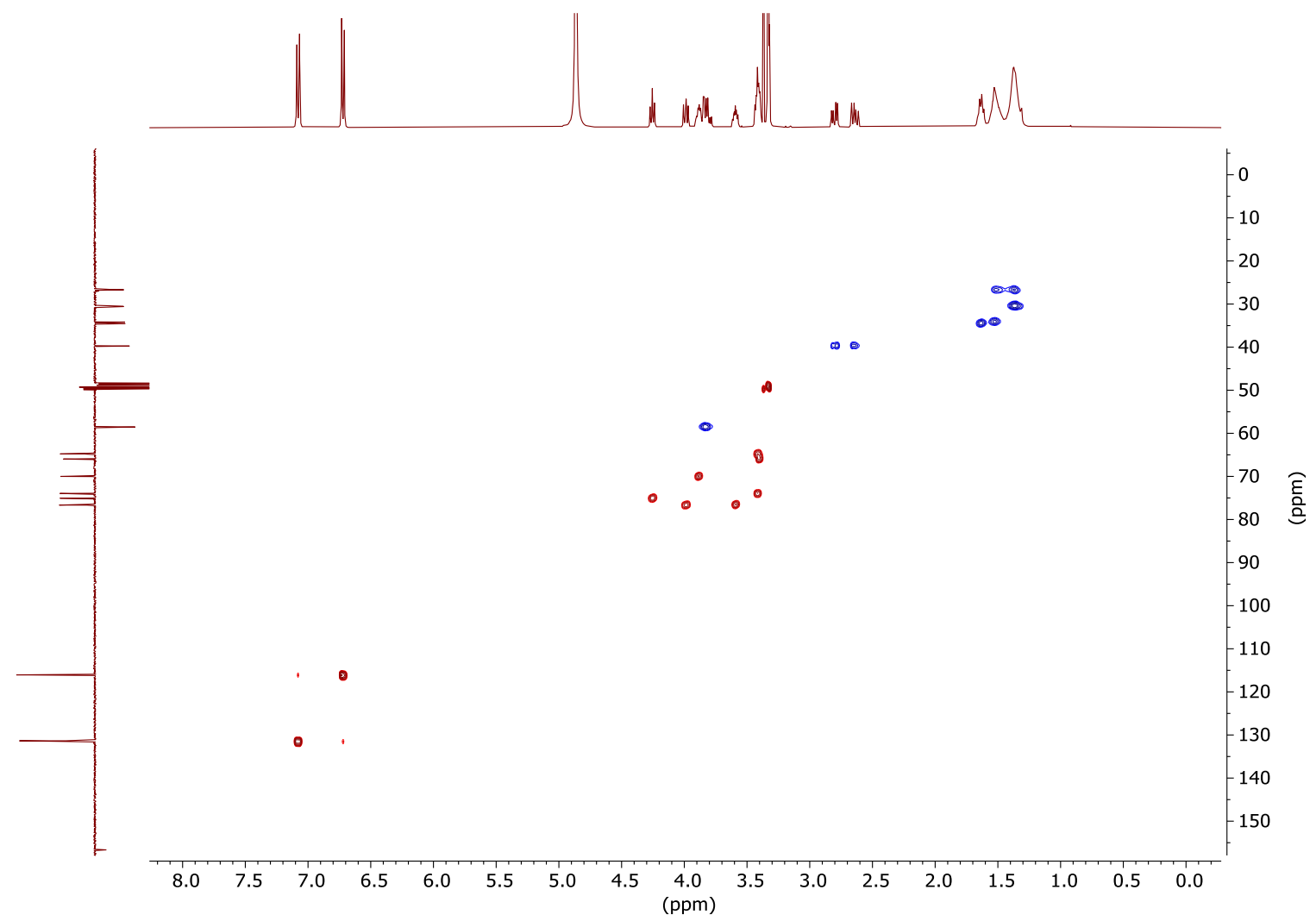

Figure S51. HSQC spectrum (400.16, $\left.100.63 \mathrm{MHz}, \mathrm{CD}_{3} \mathrm{OD}\right)$ of 2a.TFA. 


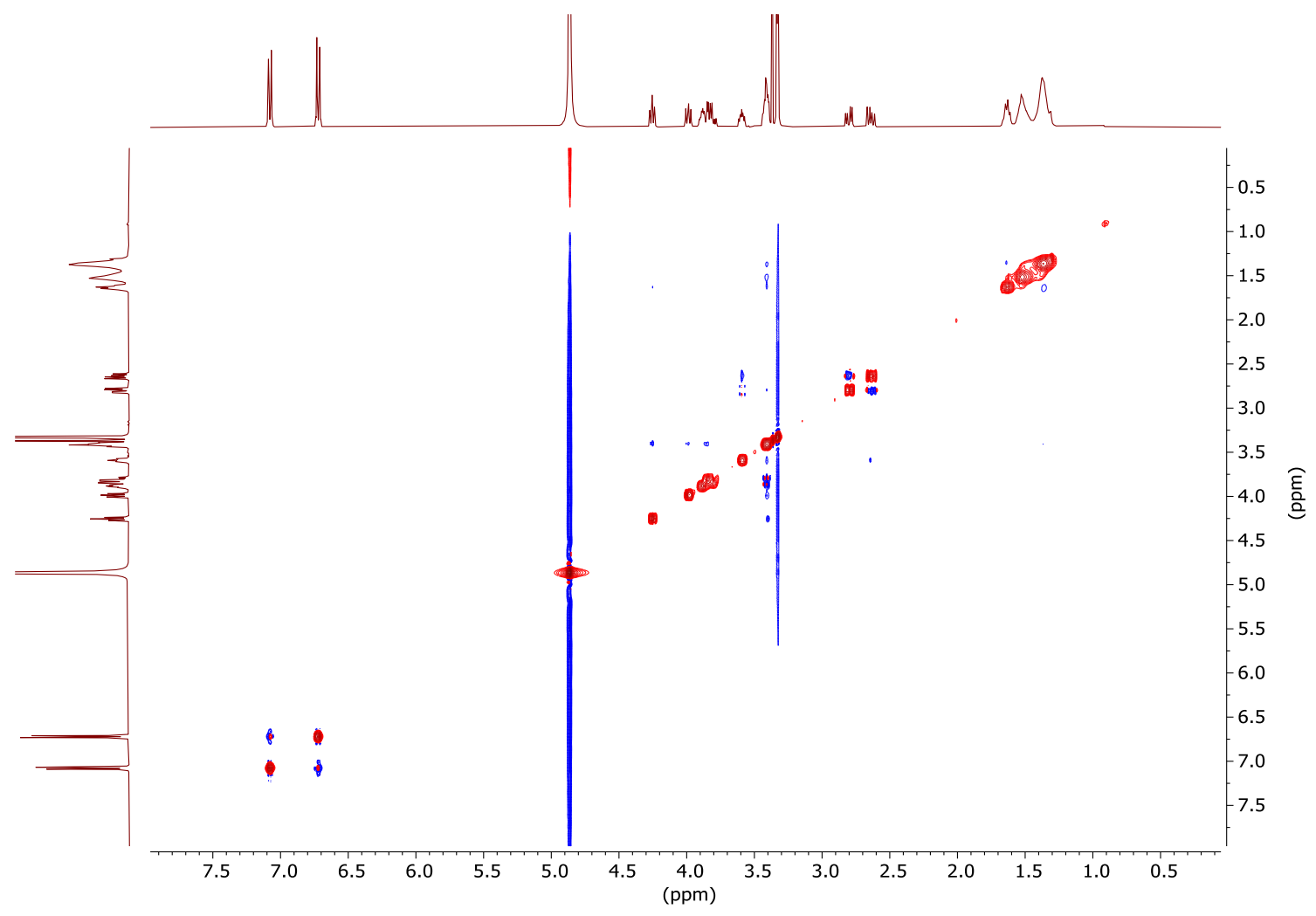

Figure S52. 2D NOESY spectrum (400.16 MHz, $\left.\mathrm{CD}_{3} \mathrm{OD}\right)$ of $\mathbf{2 a} \cdot \mathrm{TFA}$.

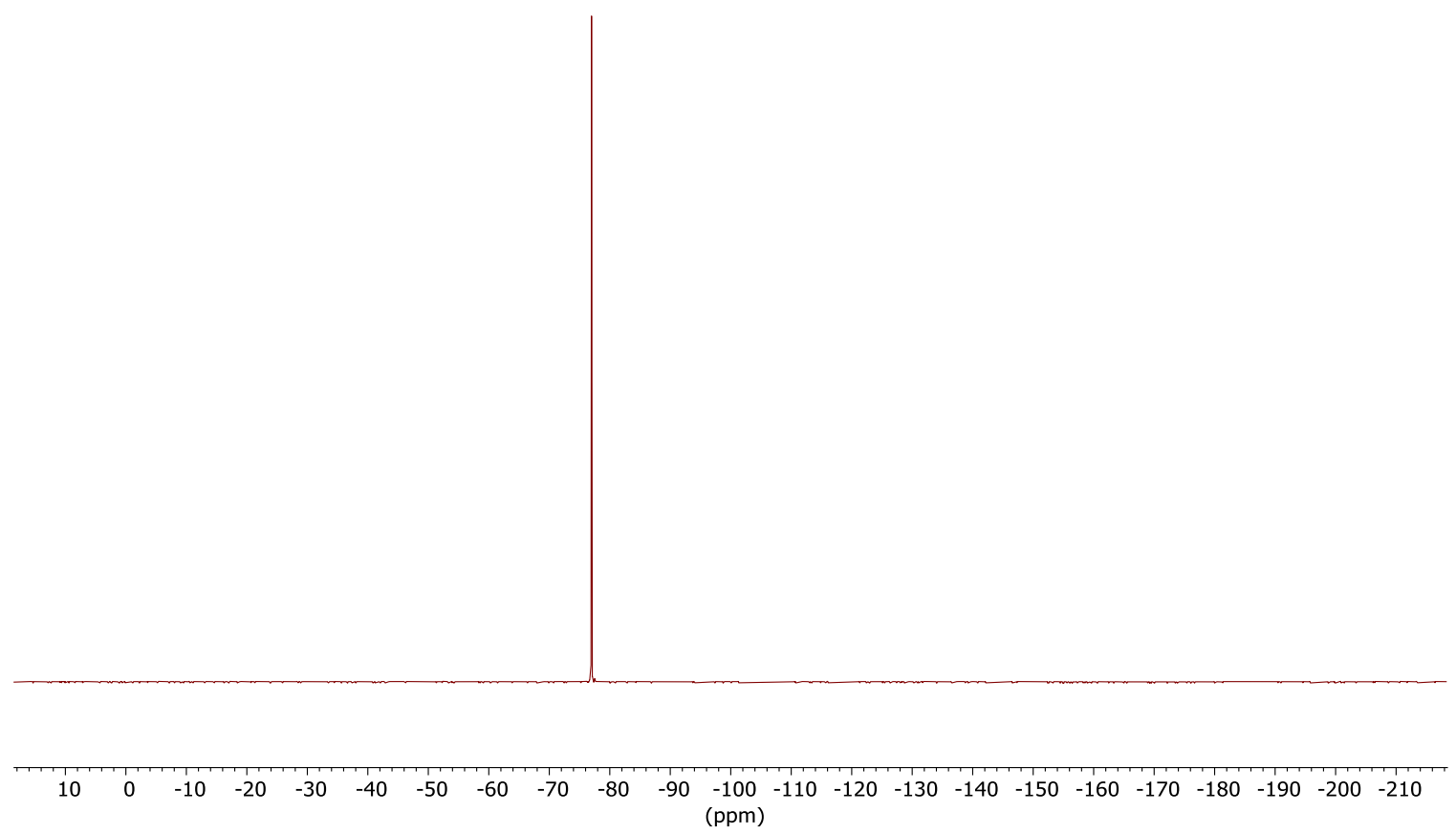

Figure S53. ${ }^{19} \mathrm{~F}$ NMR spectrum $\left(376.49 \mathrm{MHz}, \mathrm{CD}_{3} \mathrm{OD}\right)$ of 2a.TFA. 


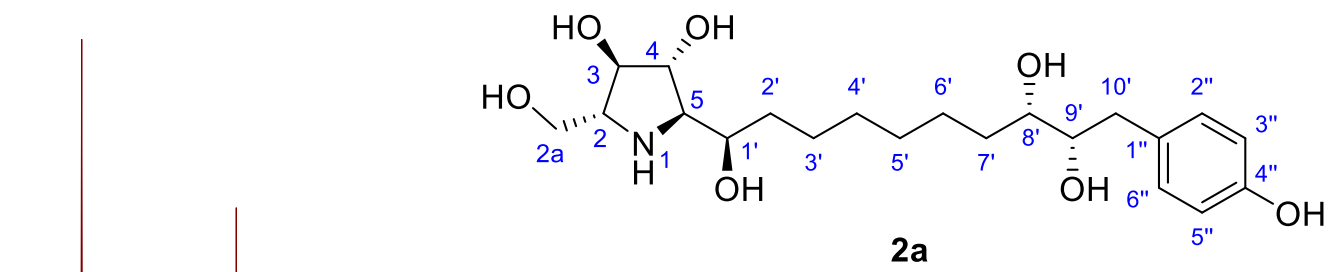

$2 a$
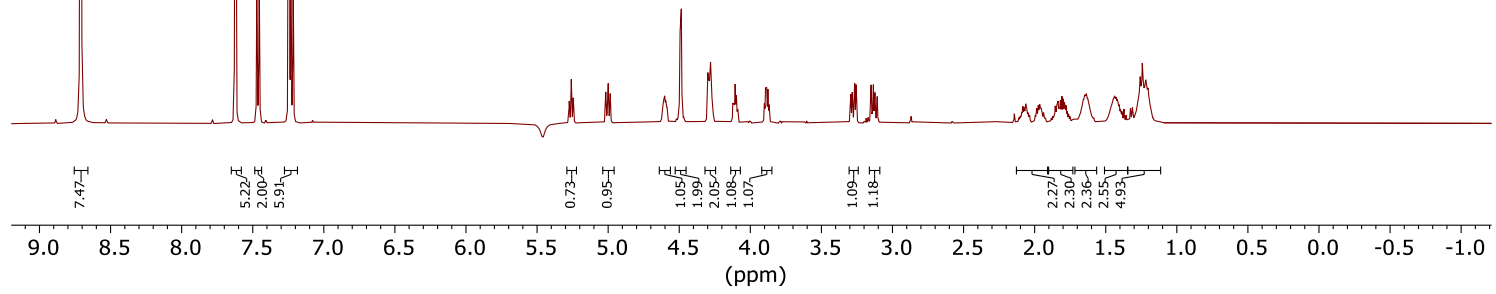

Figure S54. ${ }^{1} \mathrm{H}$ NMR spectrum (499.67 MHz, $\mathrm{C}_{5} \mathrm{D}_{5} \mathrm{~N}$, water suppression) of 2a.TFA.
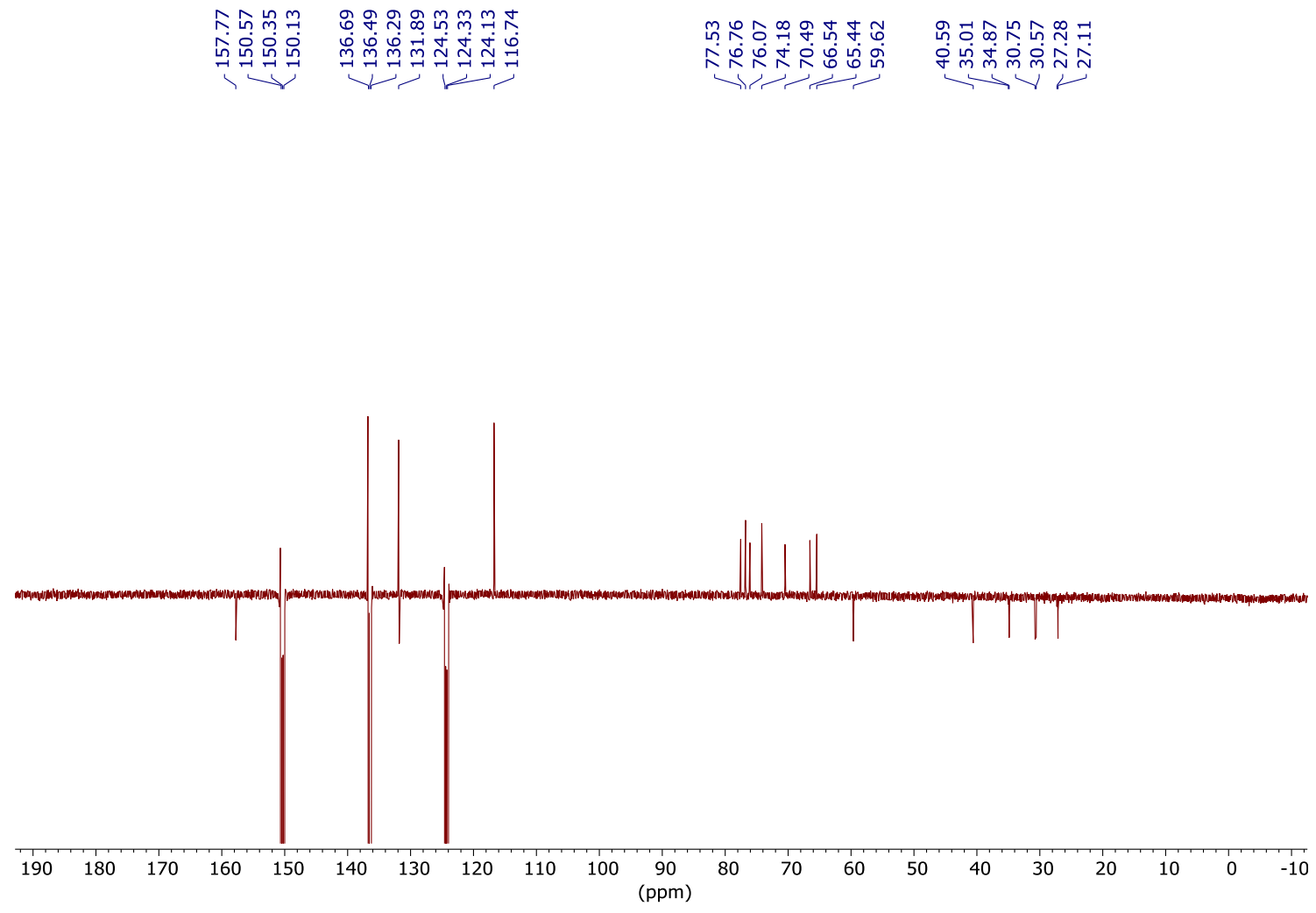

Figure S55. DEPTQ spectrum (125.67 MHz, $\left.\mathrm{C}_{5} \mathrm{D}_{5} \mathrm{~N}\right)$ of 2a.TFA. 


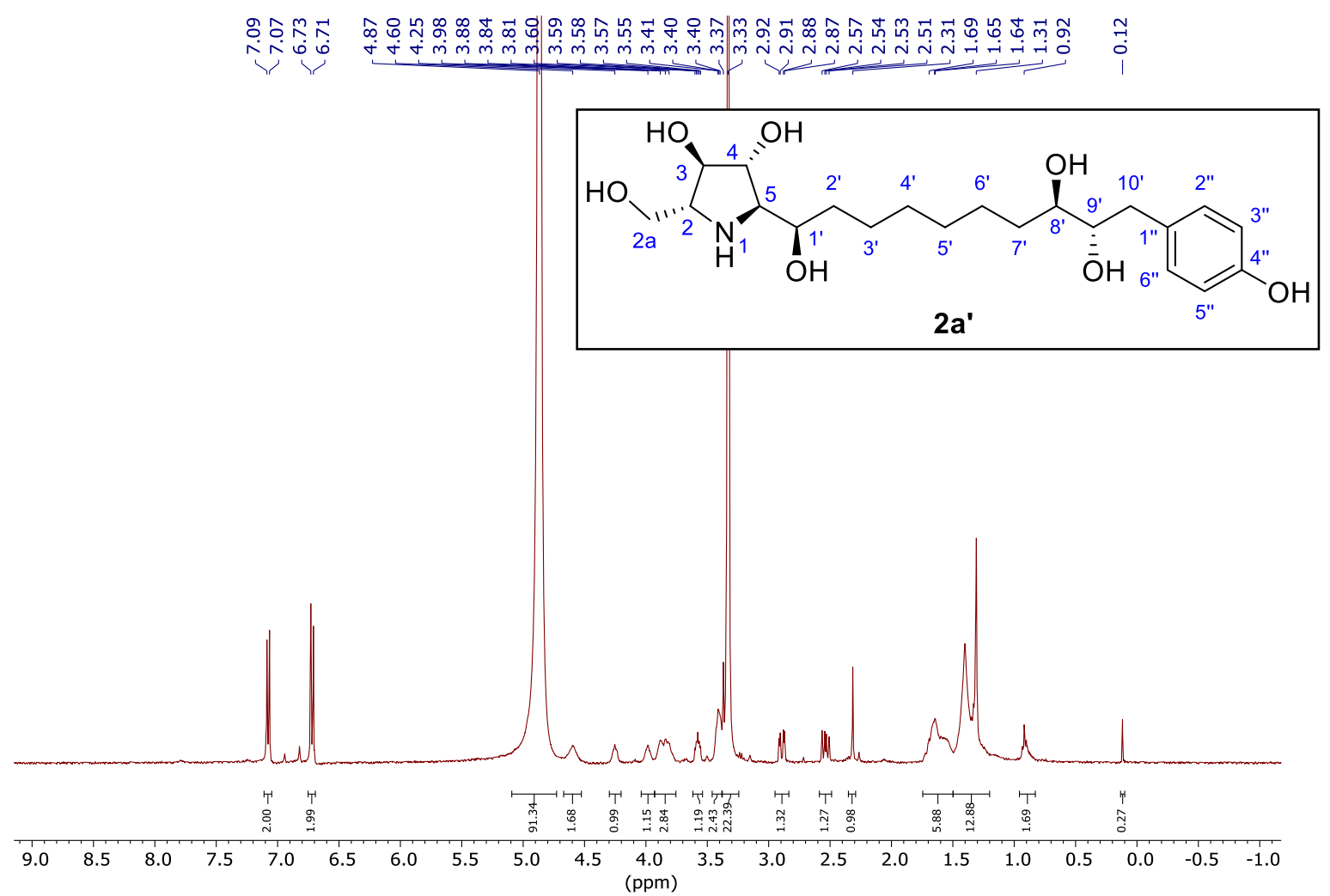

Figure S56. ${ }^{1} \mathrm{H}$ NMR spectrum $\left(400.16 \mathrm{MHz}, \mathrm{CD}_{3} \mathrm{OD}\right)$ of $\mathbf{2 a}$ '.TFA.
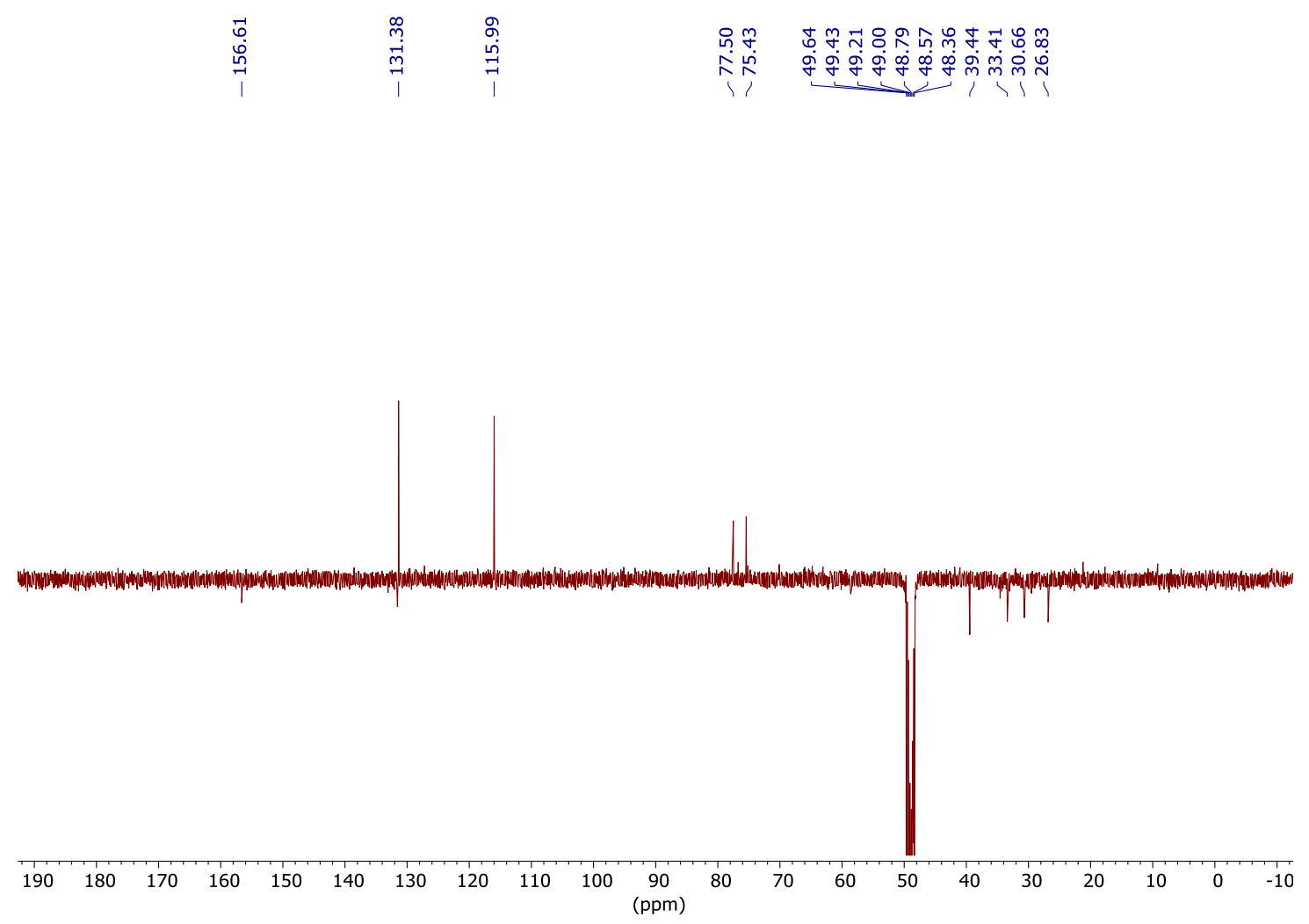

Figure S57. DEPTQ spectrum (100.63 MHz, $\left.\mathrm{CD}_{3} \mathrm{OD}\right)$ of $\mathbf{2 a}^{\prime} \cdot \mathrm{TFA}$. 


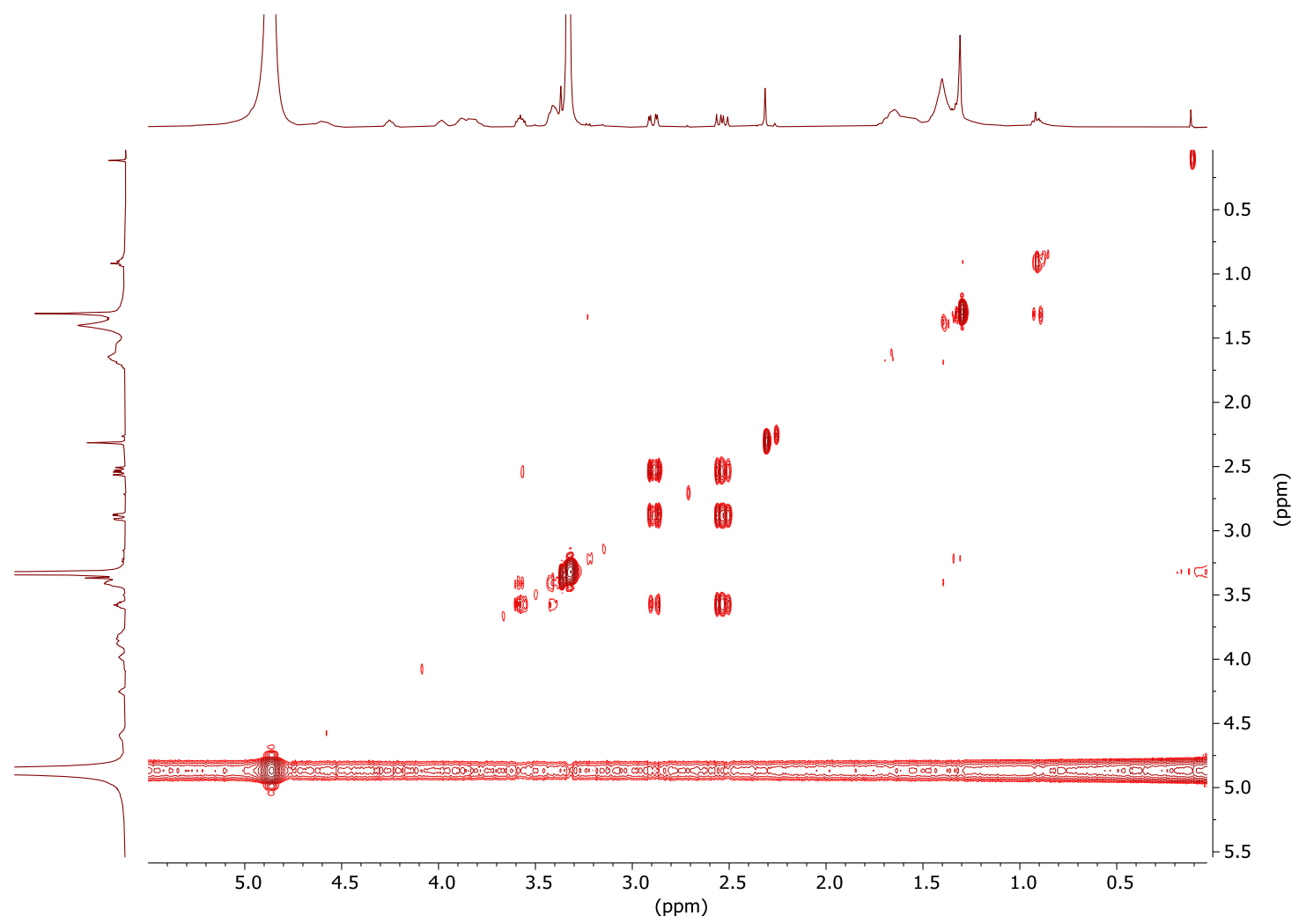

Figure S58. COSY spectrum (400.16 MHz, $\left.\mathrm{CD}_{3} \mathrm{OD}\right)$ of $\mathbf{2 a}^{\prime} \cdot \mathrm{TFA}$.

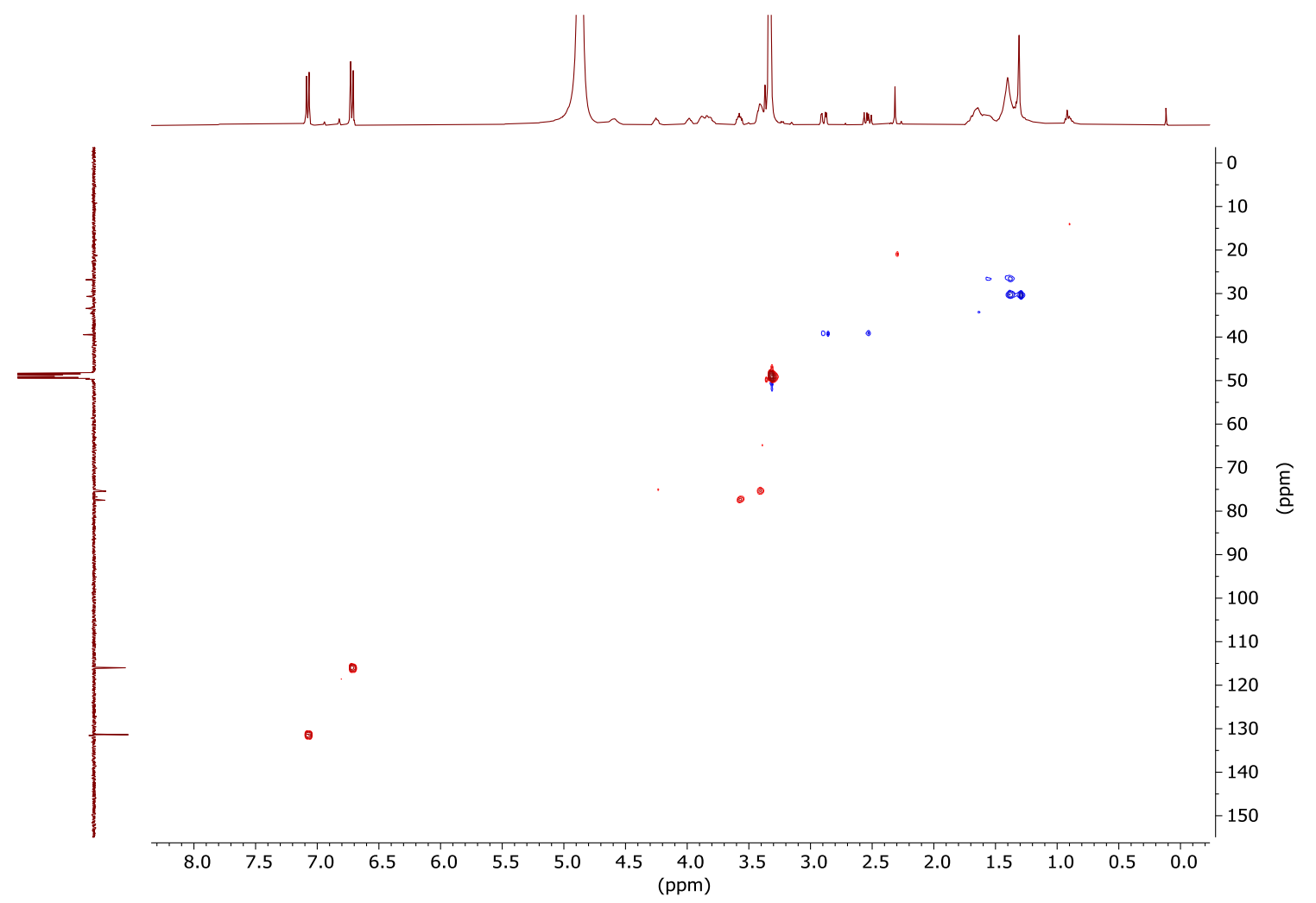

Figure S59. HSQC spectrum $\left(400.16,100.67 \mathrm{MHz}, \mathrm{CD}_{3} \mathrm{OD}\right)$ of $\mathbf{2 a}^{\prime} \cdot \mathrm{TFA}$. 


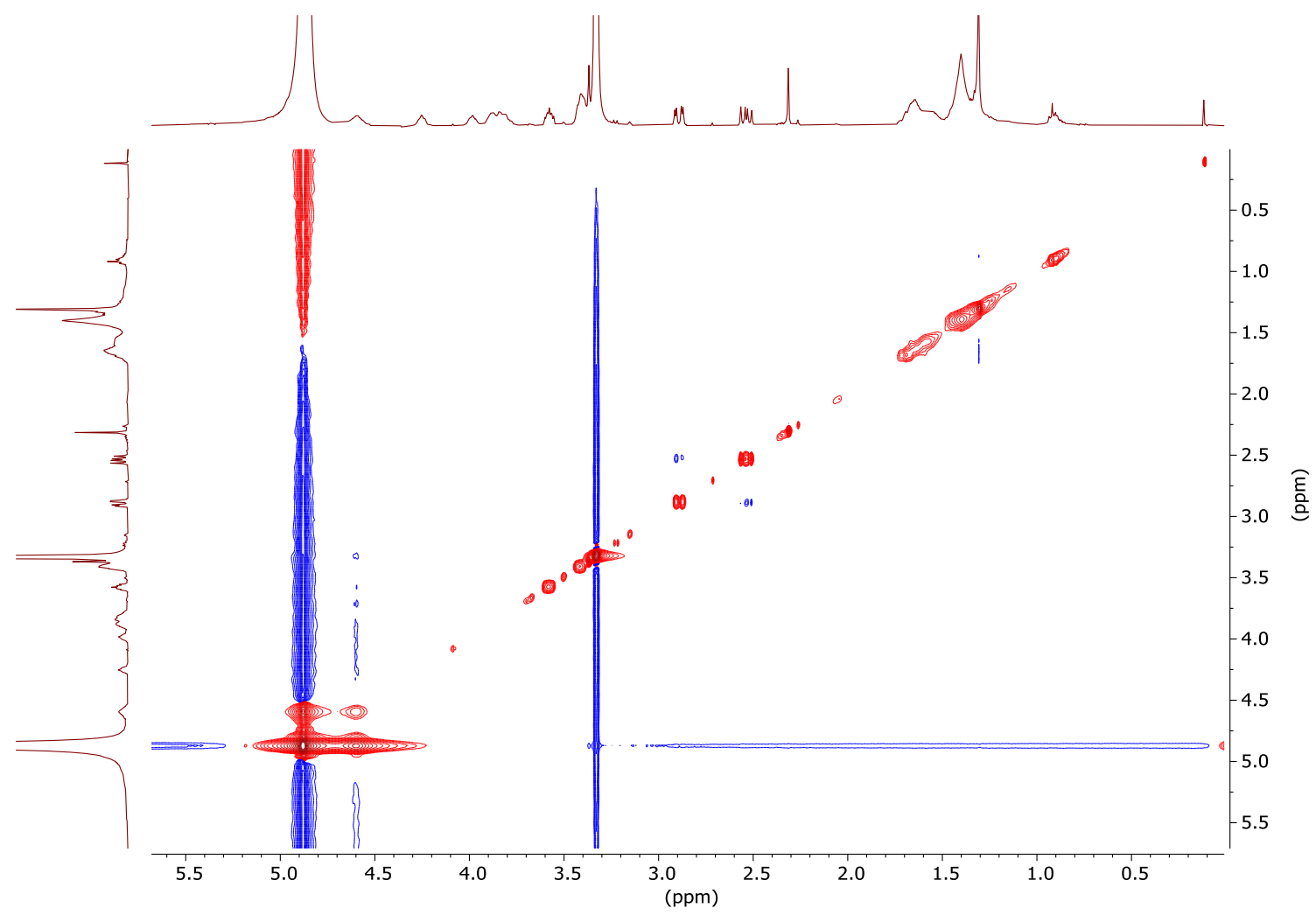

Figure S60. 2D NOESY spectrum (400.16 MHz, CD $\left.{ }_{3} \mathrm{OD}\right)$ of $\mathbf{2 a}^{\prime} \cdot$ TFA.

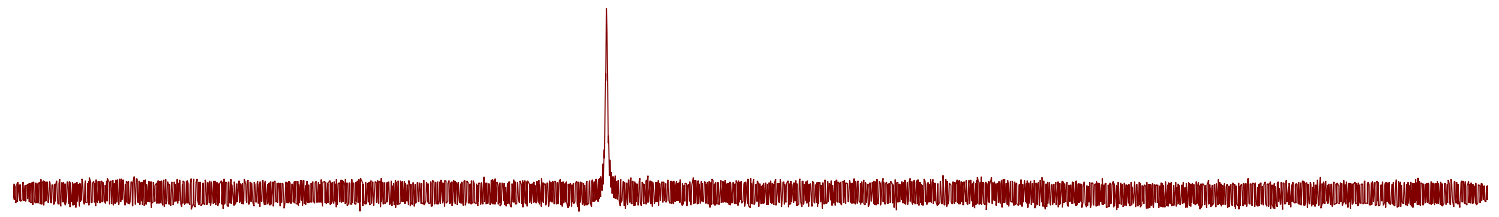

$\begin{array}{llllllllllllllllllllllllll}10 & 0 & -10 & -20 & -30 & -40 & -50 & -60 & -70 & -80 & -90 & -100 & -110 & -120 & -130 & -140 & -150 & -160 & -170 & -180 & -190 & -200 & -210\end{array}$

Figure S61. ${ }^{19} \mathrm{~F}$ NMR spectrum $\left(376.49 \mathrm{MHz}, \mathrm{CD}_{3} \mathrm{OD}\right)$ of $\mathbf{2 a}^{\prime} \cdot \mathrm{TFA}$. 
Column: $\quad$ Phenomenex Gemini $5 \mu(2.0 \times 100 \mathrm{~mm})$.

Detection: $\quad \mathrm{UV}$ at $220 \mathrm{~nm}$.

Solvent: $\quad \mathrm{H}_{2} \mathrm{O}, 0.0025 \% \mathrm{TFA}(\mathrm{A}) ; \mathrm{MeOH}(\mathrm{B})$.

Method: $\quad 0 \% \mathrm{~B}(0.0 \rightarrow 3.0 \mathrm{~min}), 11 \rightarrow 16 \% \mathrm{~B}(3.0 \rightarrow 18.0 \mathrm{~min}), 0 \% \mathrm{~B}(18.0 \rightarrow 23.0 \mathrm{~min})$.

Flow rate: $\quad 0.2 \mathrm{~mL} / \mathrm{min}$.

(a) Before semi-preparative HPLC (2a·TFA/2a'·TFA).

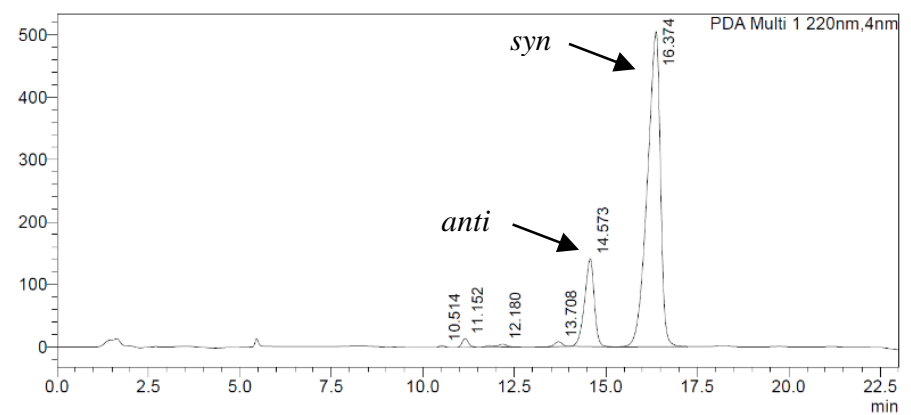

(b) After semi-preparative HPLC (2a-TFA).

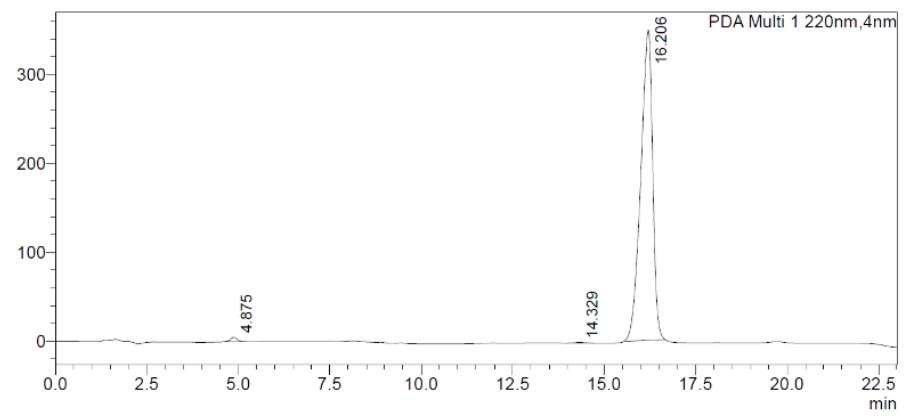

(c) After semi-preparative HPLC (2a'-TFA).

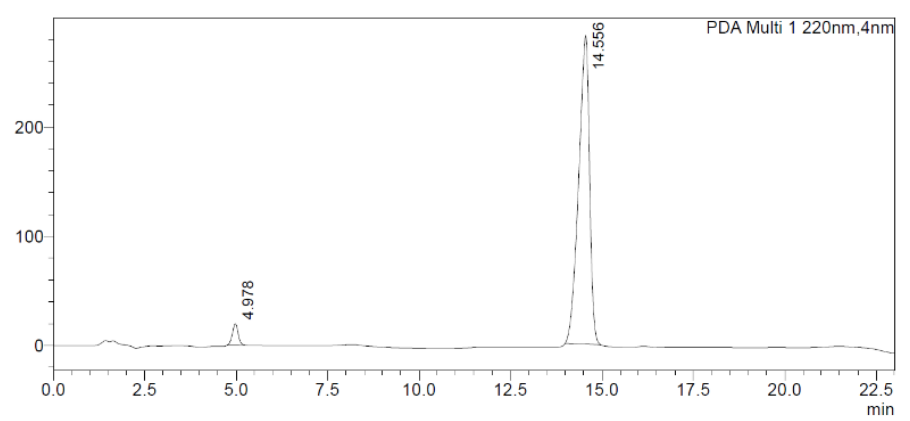

Figure S62. HPLC chromatograms of $\mathbf{2 a} \cdot$ TFA and $\mathbf{2} \mathbf{a}^{\prime} \cdot$ TFA.

\begin{tabular}{|c|c|c|}
\hline$R_{\mathbf{t}}(\min )$ & Area (\%) & Attribution \\
\hline 10.514 & 0.215 & Impurity \\
\hline 11.152 & 1.101 & Impurity \\
\hline 12.180 & 0.659 & Impurity \\
\hline 13.708 & 0.734 & Impurity \\
\hline 14.573 & 16.658 & $\mathbf{2} \mathbf{a}^{\prime} \cdot \mathrm{TFA}$ \\
\hline 16.374 & 80.638 & $\mathbf{2 a} \cdot \mathrm{TFA}$ \\
\hline
\end{tabular}

\begin{tabular}{rrc}
$\boldsymbol{R}_{\mathbf{t}}(\mathbf{m i n})$ & Area $(\%)$ & Attribution \\
4.875 & 0.838 & Impurity \\
14.329 & 0.177 & $\mathbf{2} \mathbf{a}^{\prime} \cdot \mathrm{TFA}$ \\
16.206 & 98.985 & $\mathbf{2 a} \cdot \mathrm{TFA}$ \\
\hline
\end{tabular}

\begin{tabular}{ccc}
$\boldsymbol{R}_{\mathbf{t}}(\mathbf{m i n})$ & Area $(\boldsymbol{\%})$ & Attribution \\
4.978 & 3.483 & Impurity \\
14.556 & 96.517 & $\mathbf{2 a}^{\prime} \cdot \mathrm{TFA}$ \\
\hline
\end{tabular}




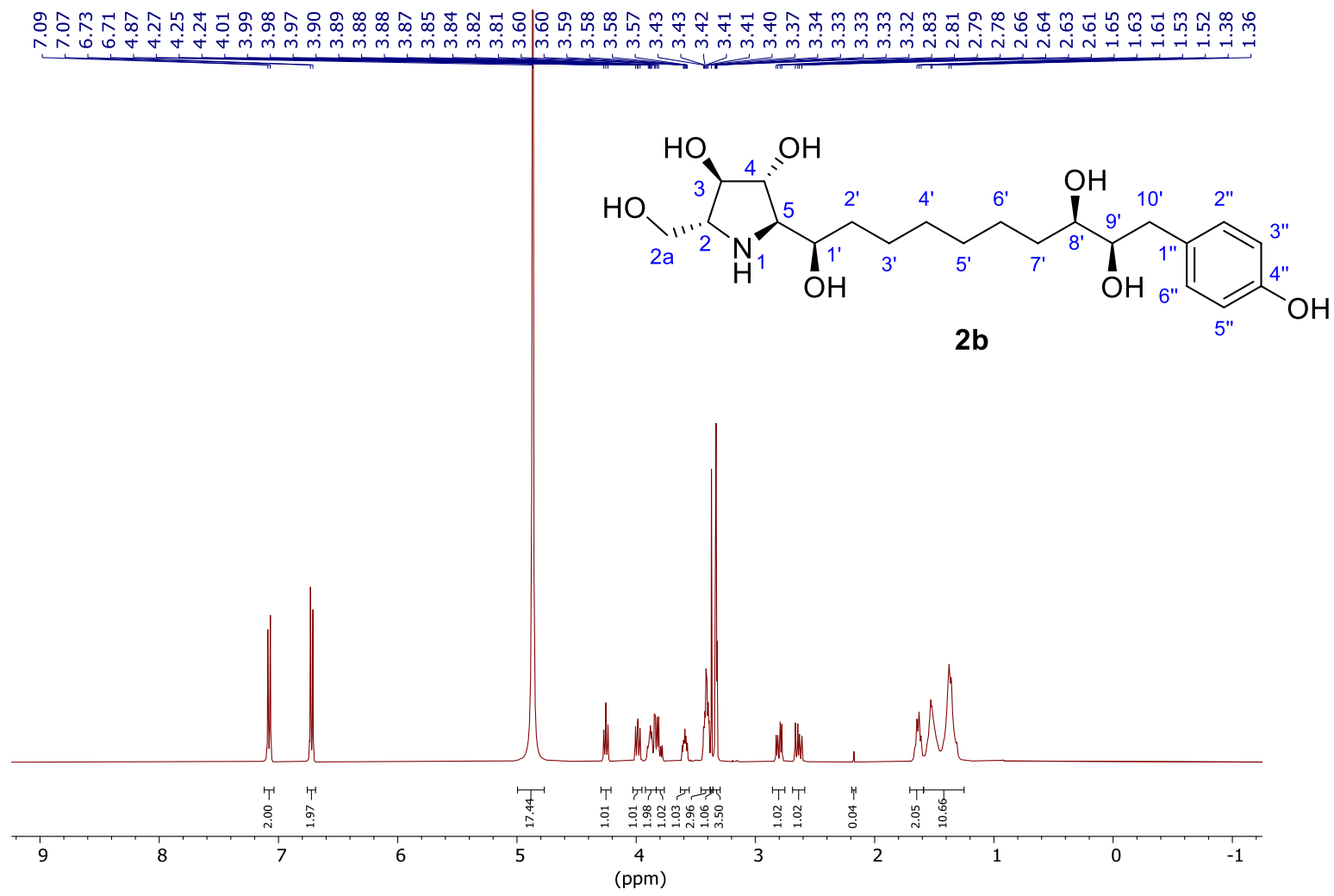

Figure S63. ${ }^{1} \mathrm{H}$ NMR spectrum $\left(400.16 \mathrm{MHz}, \mathrm{CD}_{3} \mathrm{OD}\right)$ of $\mathbf{2 b} \cdot \mathrm{TFA}$.
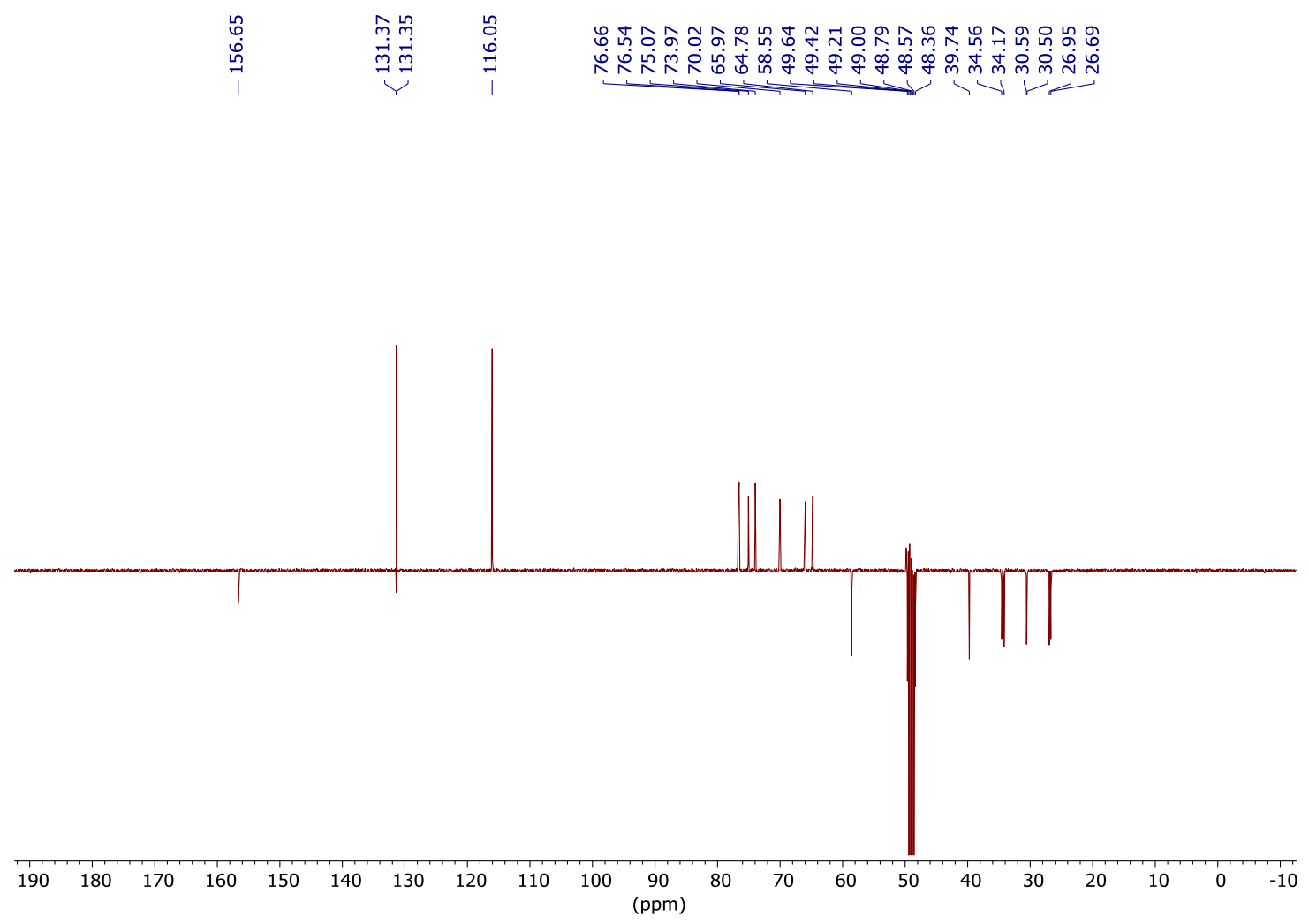

Figure S64. DEPTQ spectrum (100.63 MHz, $\left.\mathrm{CD}_{3} \mathrm{OD}\right)$ of $\mathbf{2 b} \cdot \mathrm{TFA}$. 


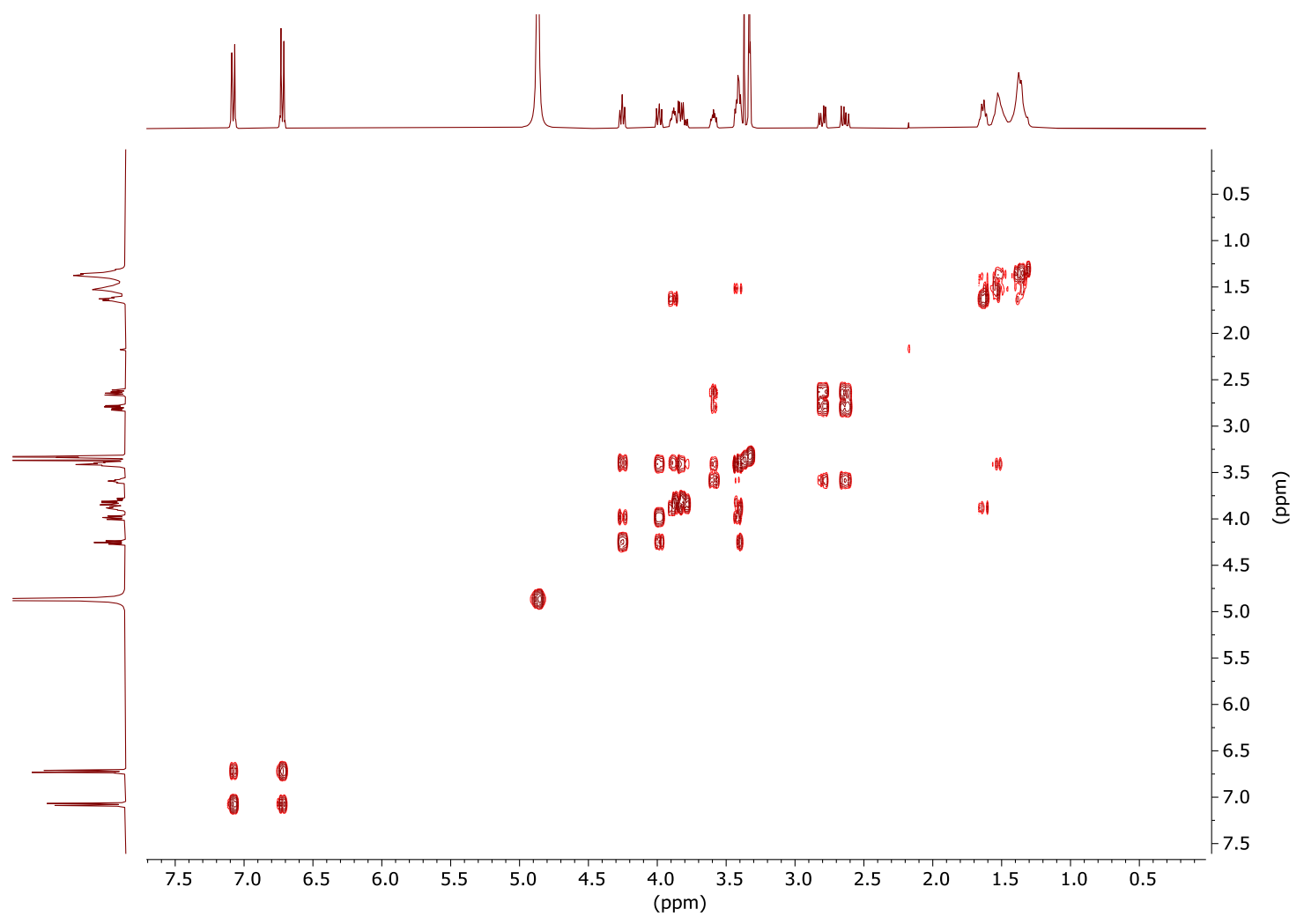

Figure S65. COSY spectrum (400.16 MHz, $\left.\mathrm{CD}_{3} \mathrm{OD}\right)$ of $\mathbf{2 b} \cdot \mathrm{TFA}$.

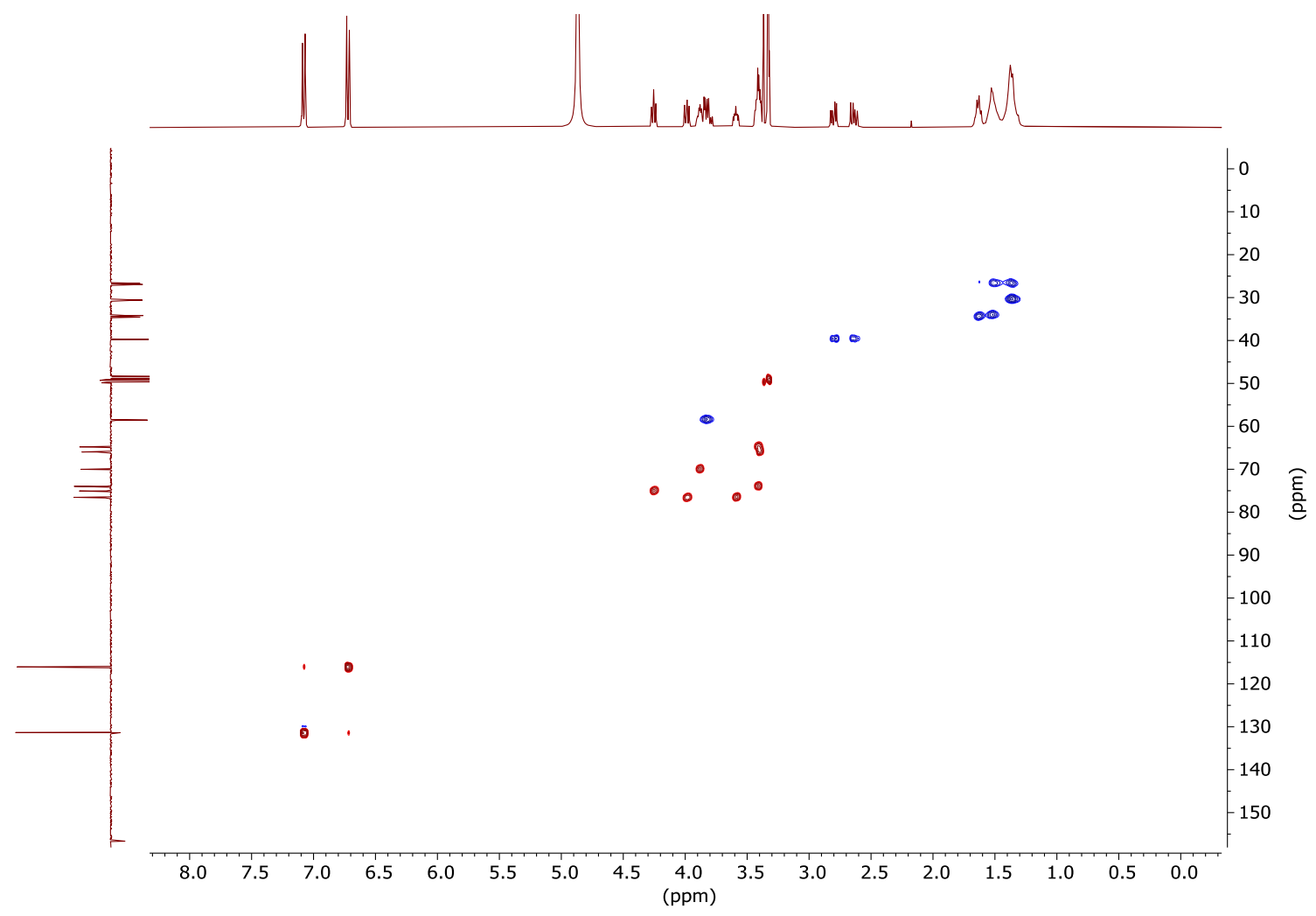

Figure S66. HSQC spectrum $\left(400.16,100.63 \mathrm{MHz}, \mathrm{CD}_{3} \mathrm{OD}\right)$ of $\mathbf{2 b} \cdot \mathrm{TFA}$. 


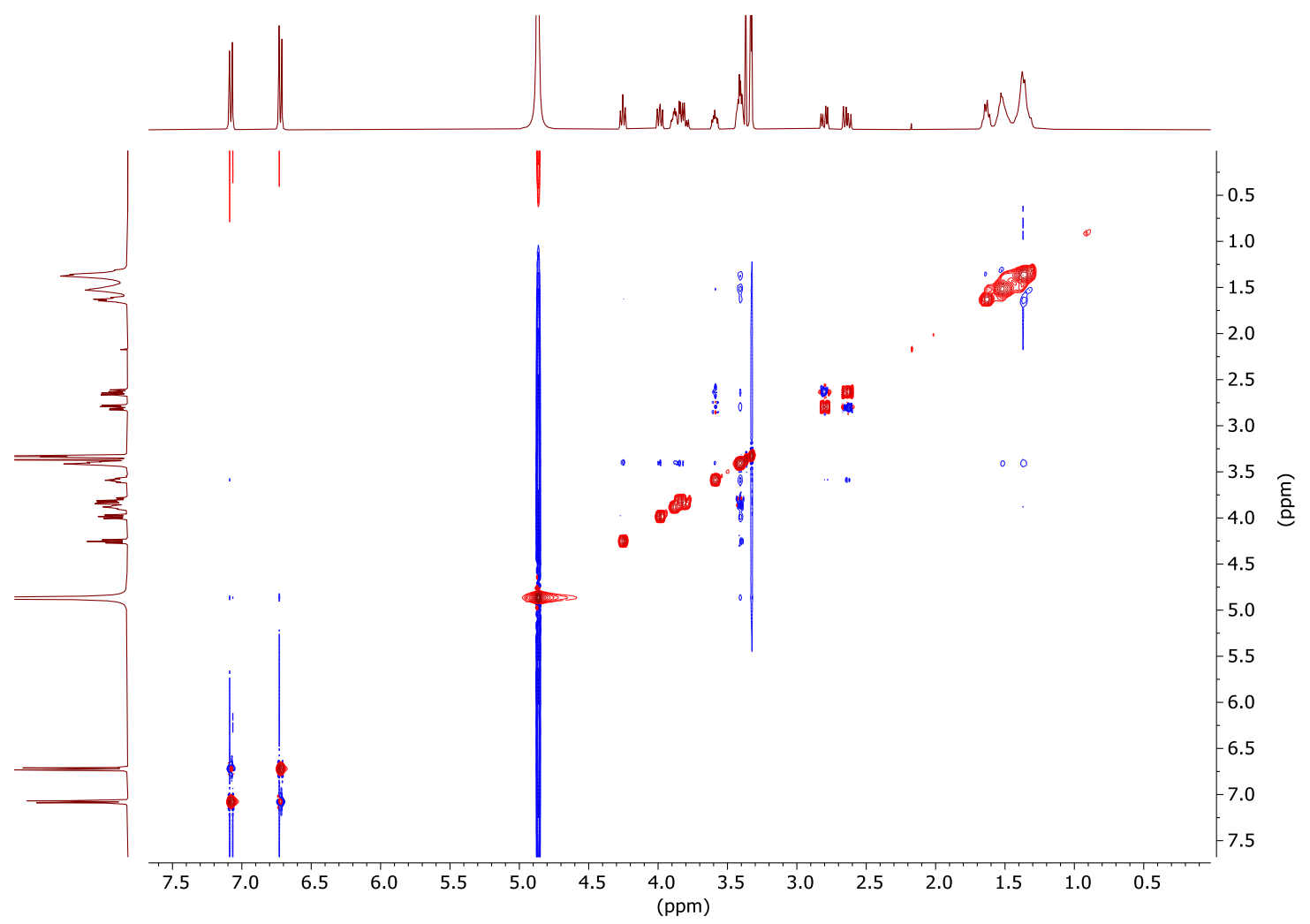

Figure S67. 2D NOESY spectrum (400.16 MHz, $\left.\mathrm{CD}_{3} \mathrm{OD}\right)$ of $\mathbf{2 b}$. TFA.

$$
\text { مू }
$$

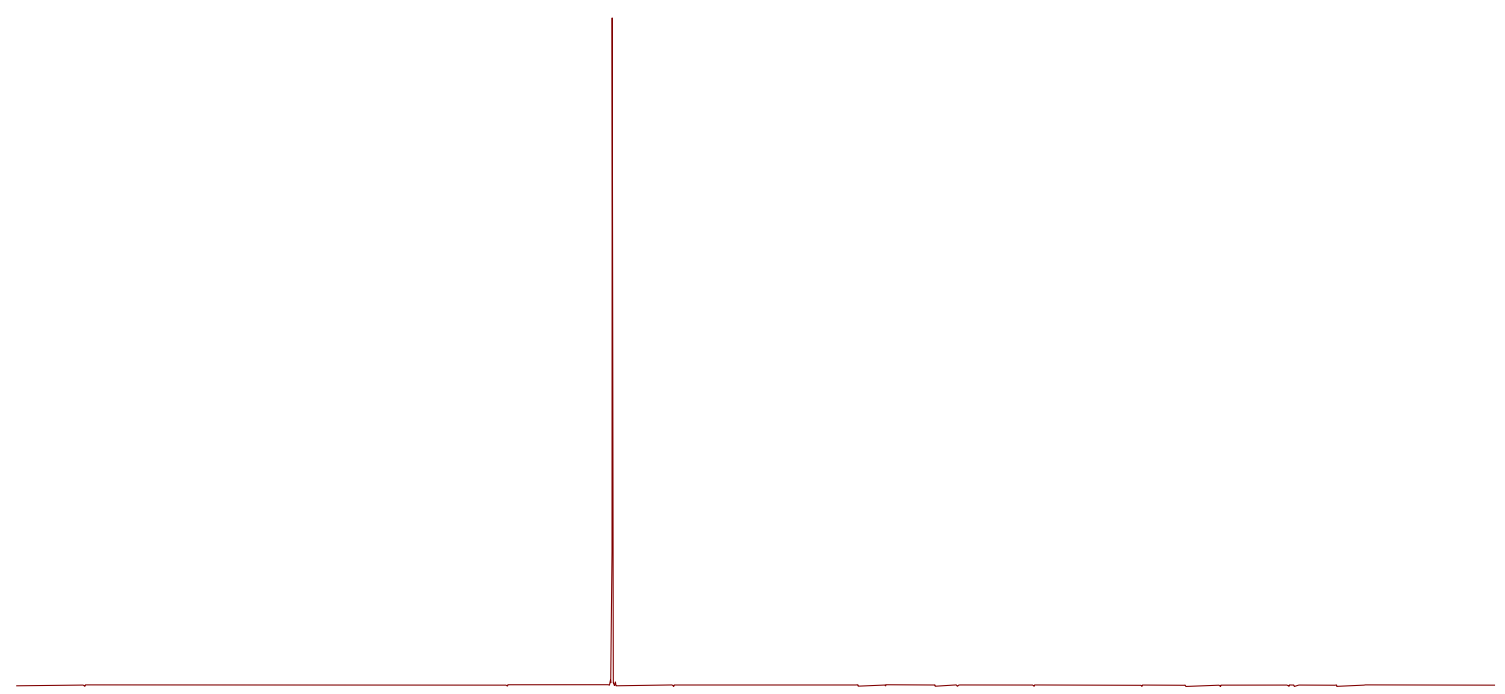

$\begin{array}{lllllllllllllllllllllllllllllllllll}10 & 0 & -10 & -20 & -30 & -40 & -50 & -60 & -70 & -80 & -90 & -100 & -110 & -120 & -130 & -140 & -150 & -160 & -170 & -180 & -190 & -200 & -210\end{array}$

Figure S68. ${ }^{19} \mathrm{~F}$ NMR spectrum $\left(376.49 \mathrm{MHz}, \mathrm{CD}_{3} \mathrm{OD}\right)$ of $\mathbf{2 b} \cdot \mathrm{TFA}$. 


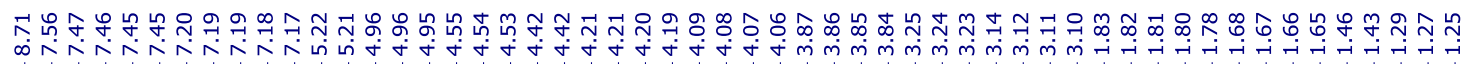

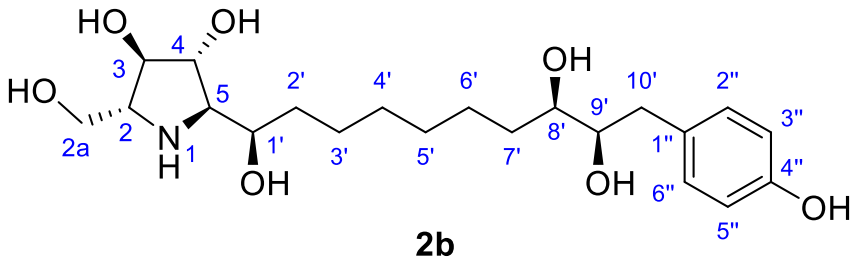

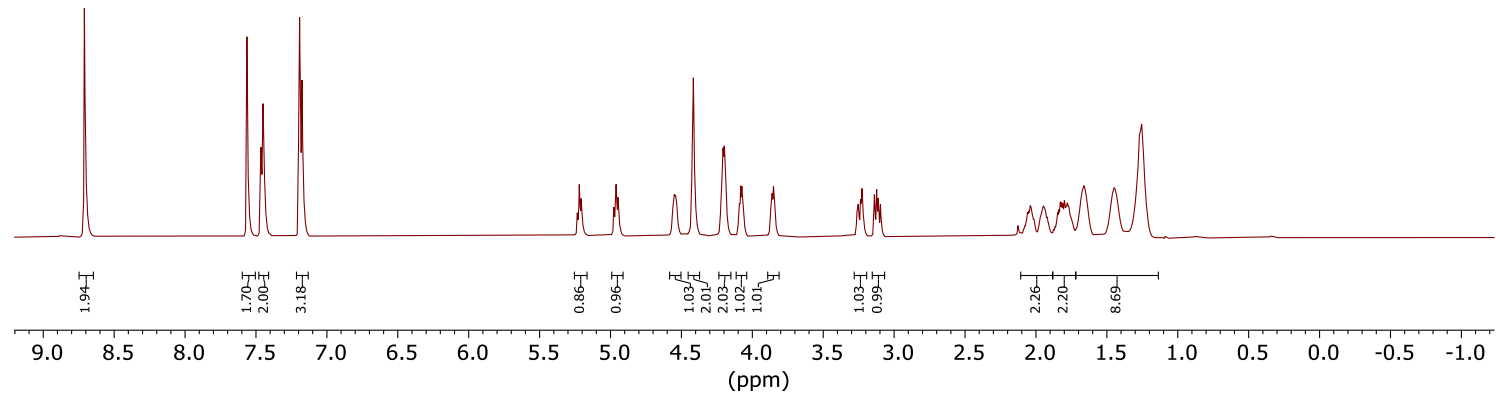

Figure S69. ${ }^{1} \mathrm{H}$ NMR spectrum (499.75 $\mathrm{MHz}, \mathrm{C}_{5} \mathrm{D}_{5} \mathrm{~N}$, water suppression) of $\mathbf{2 b} \cdot \mathrm{TFA}$.
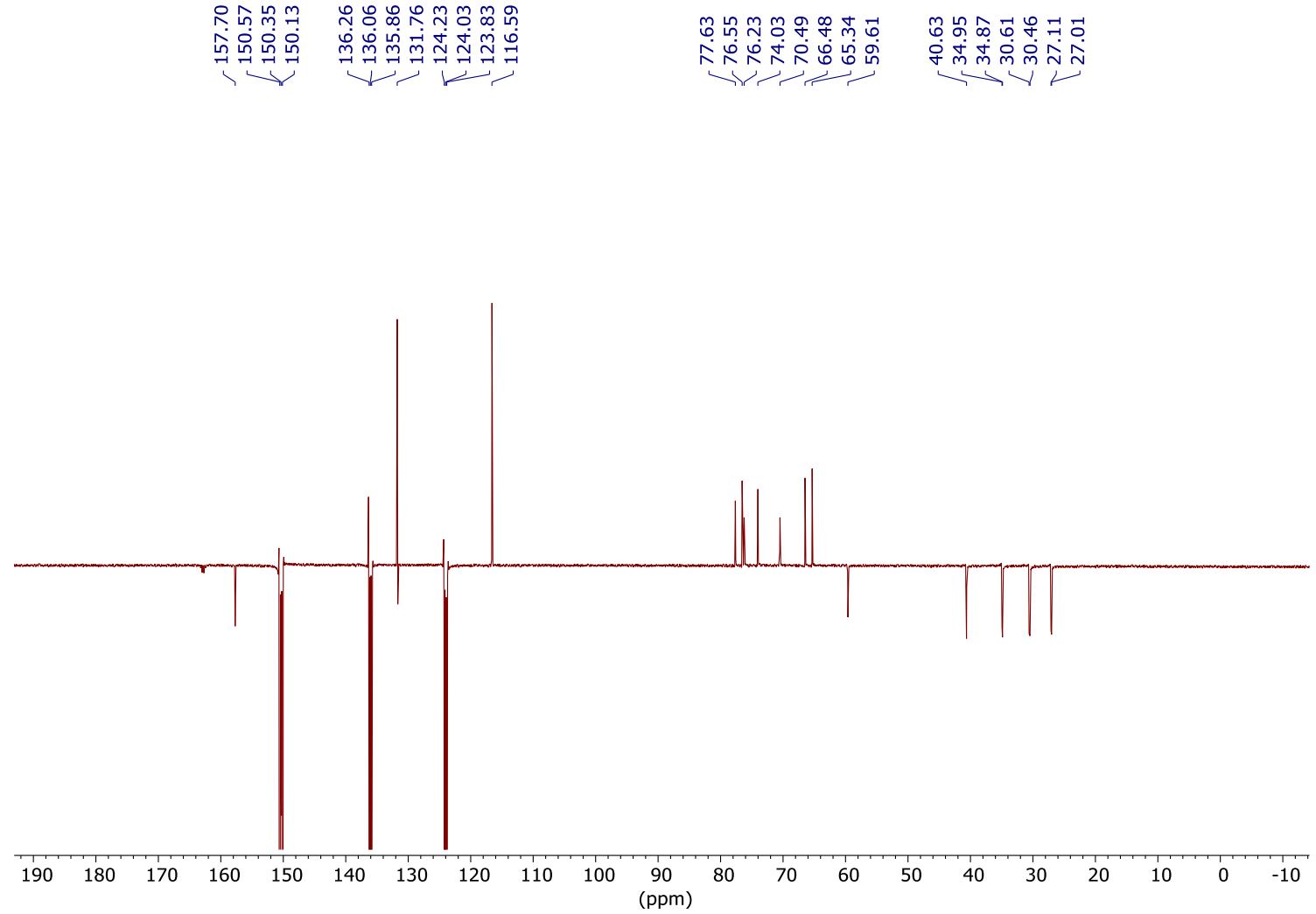

Figure S70. DEPTQ spectrum (125.67 MHz, $\left.\mathrm{C}_{5} \mathrm{D}_{5} \mathrm{~N}\right)$ of $\mathbf{2 b} \cdot$ TFA. 


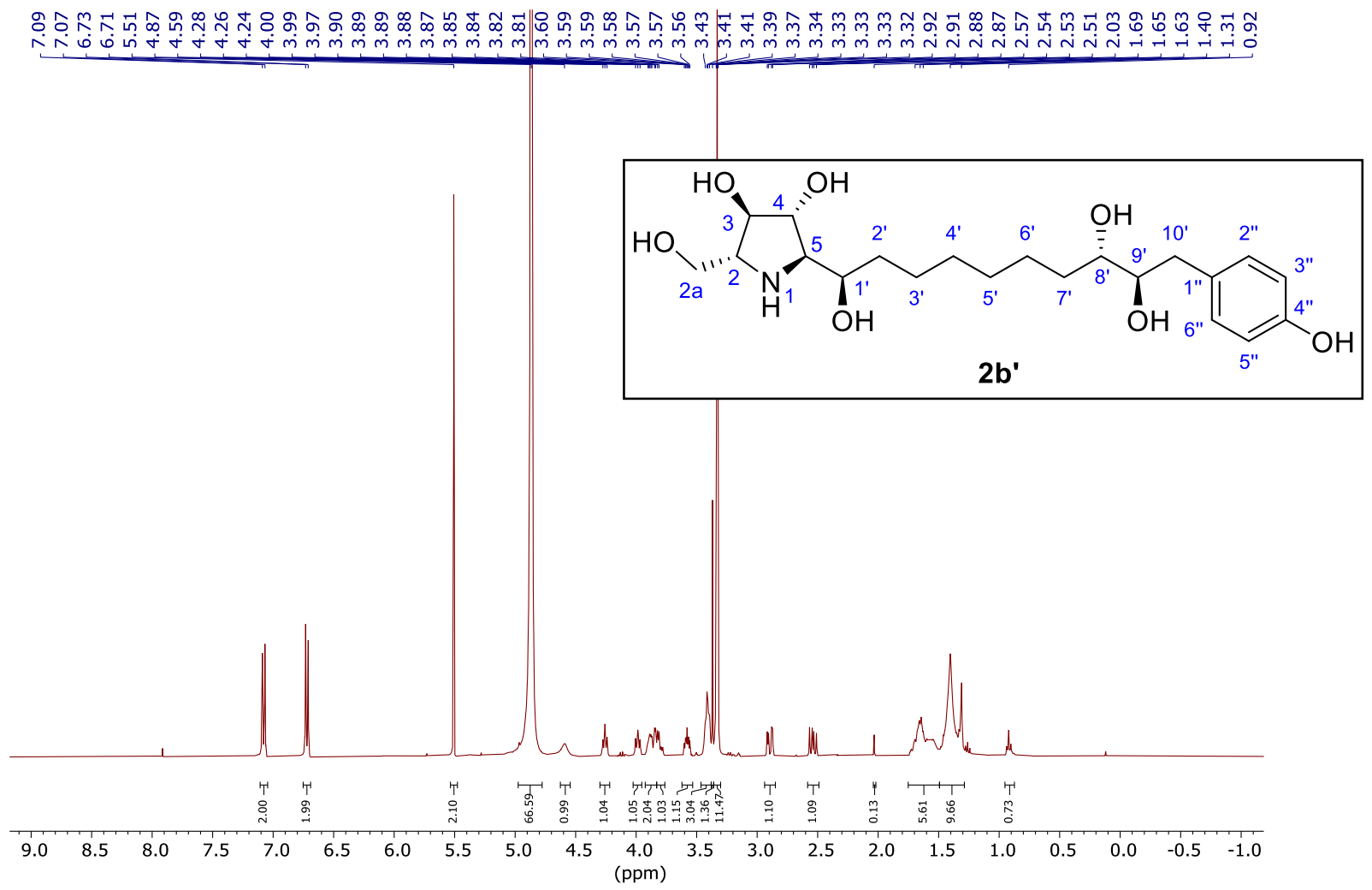

Figure S71. ${ }^{1} \mathrm{H}$ NMR spectrum $\left(400.16 \mathrm{MHz}, \mathrm{CD}_{3} \mathrm{OD}\right)$ of $\mathbf{2} \mathbf{b}^{\prime}$. TFA.
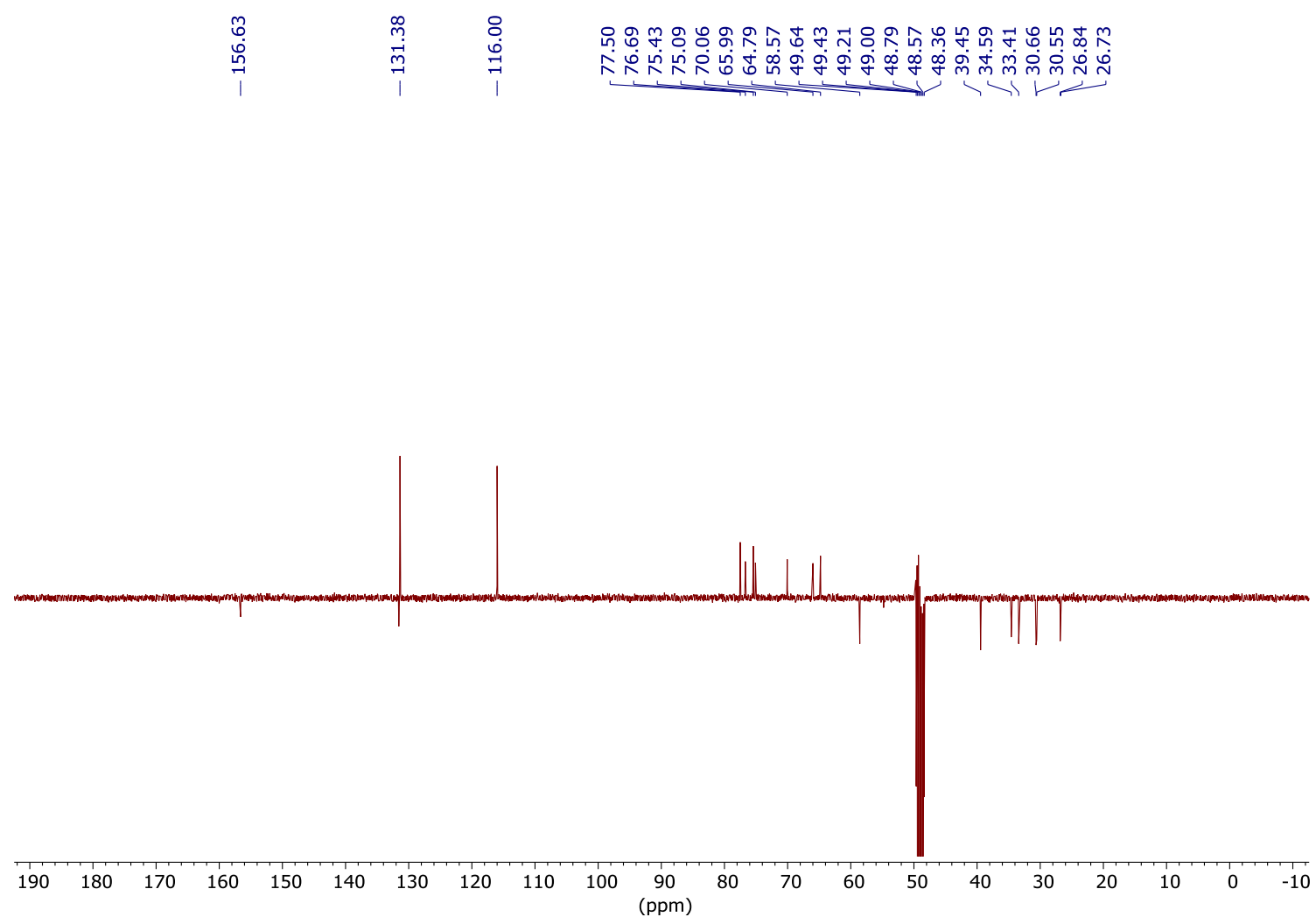

Figure S72. DEPTQ spectrum (400.16 MHz, $\left.\mathrm{CD}_{3} \mathrm{OD}\right)$ of $\mathbf{2 b}^{\prime}$.TFA. 


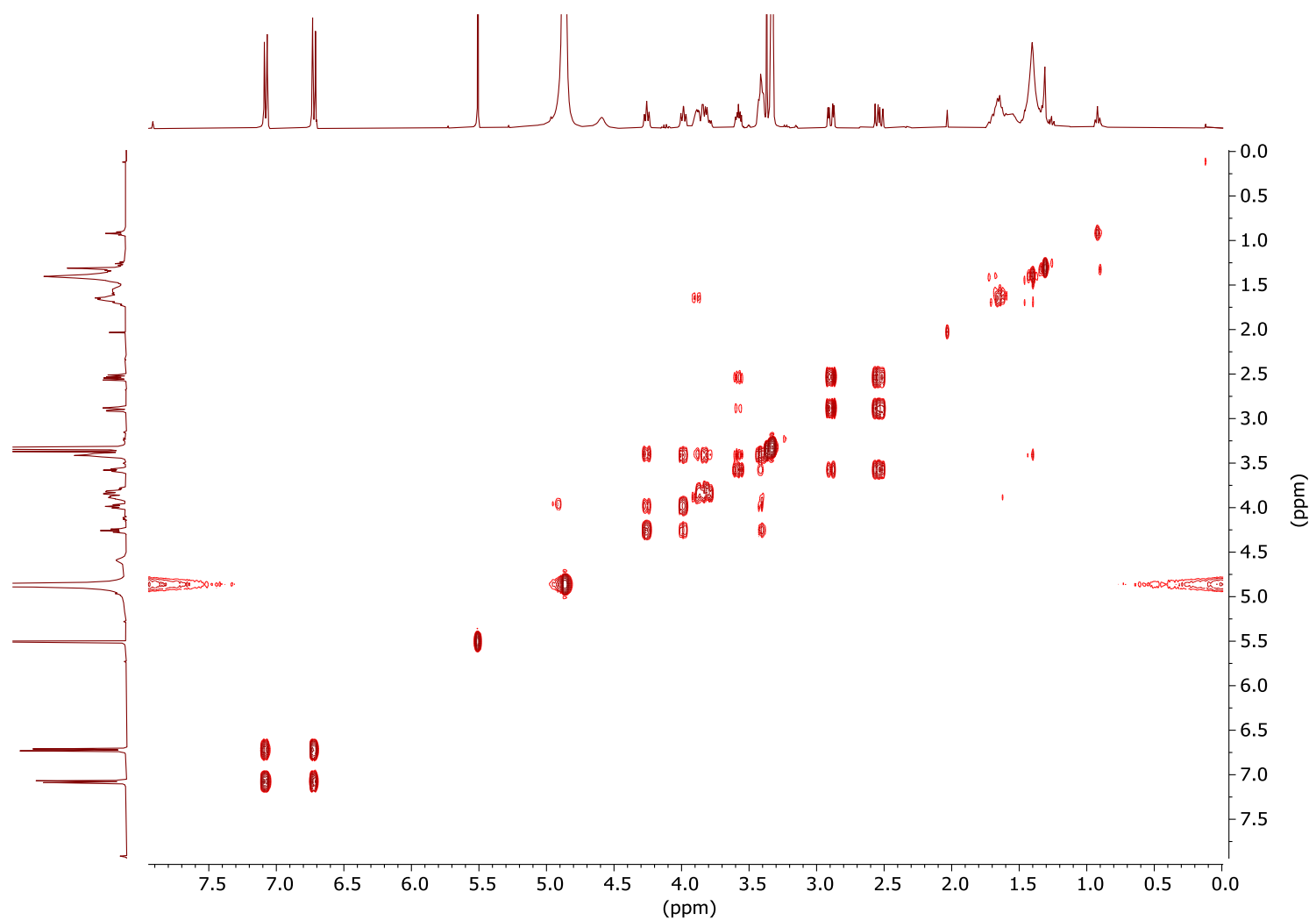

Figure S73. COSY spectrum (400.16 MHz, $\left.\mathrm{CD}_{3} \mathrm{OD}\right)$ of $\mathbf{2 b}^{\mathbf{\prime}}$. TFA.

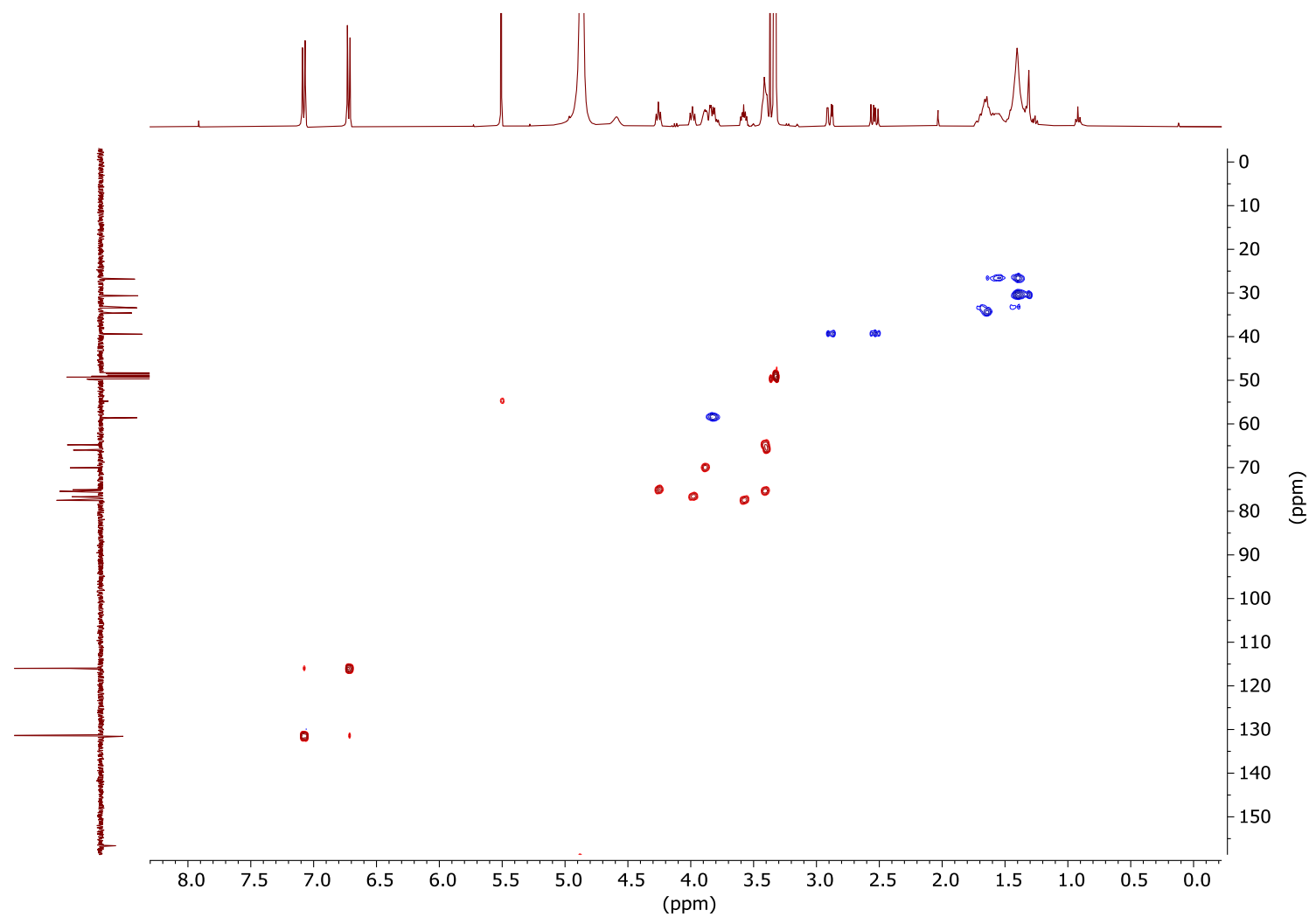

Figure S74. HSQC spectrum $\left(400.16,100.63 \mathrm{MHz}, \mathrm{CD}_{3} \mathrm{OD}\right)$ of $\mathbf{2}^{\prime}$.TFA. 


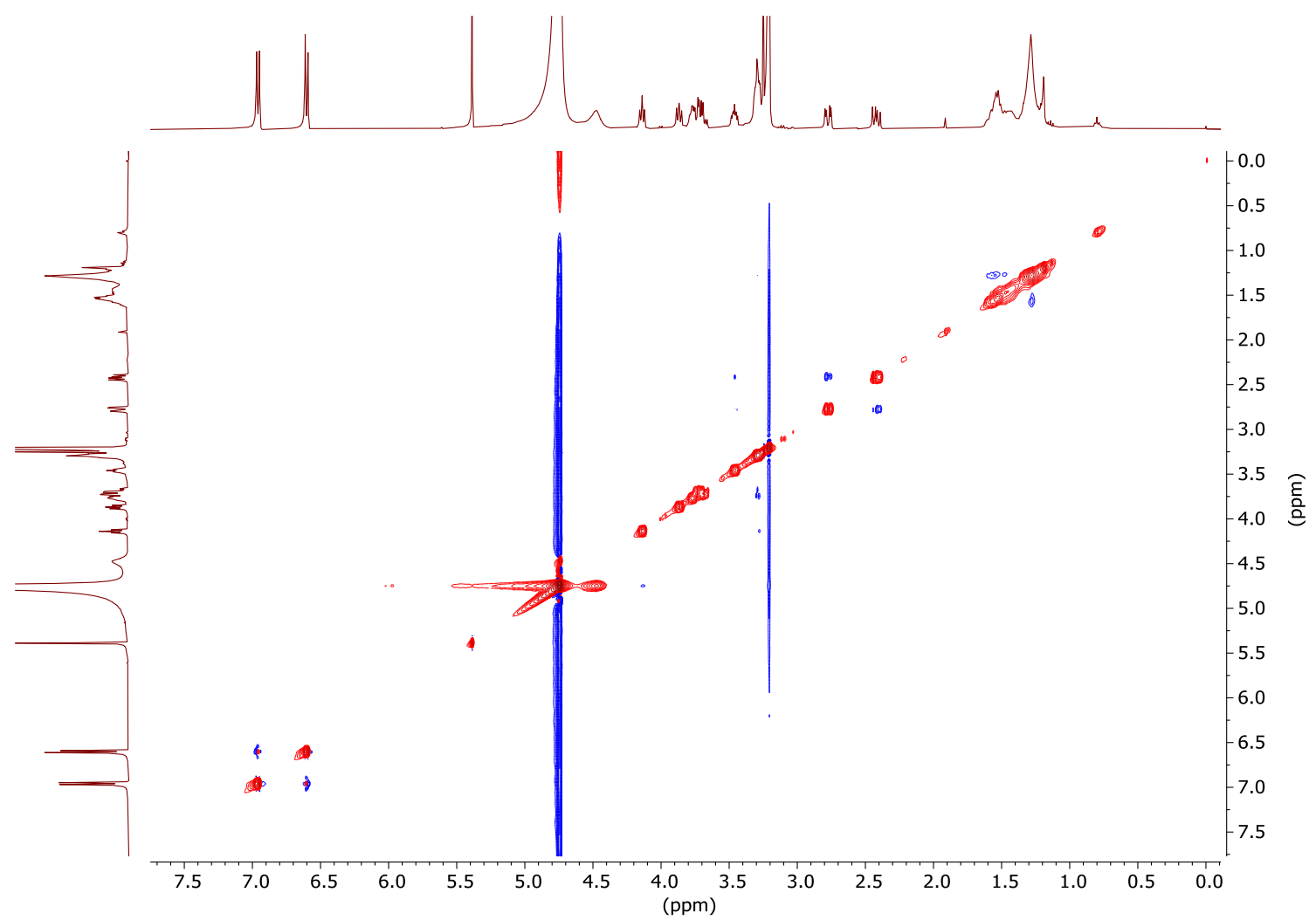

Figure S75. 2D NOESY spectrum (400.16 MHz, $\left.\mathrm{CD}_{3} \mathrm{OD}\right)$ of $\mathbf{2 b}^{\prime} \cdot$ TFA.

ลे
จิ
1

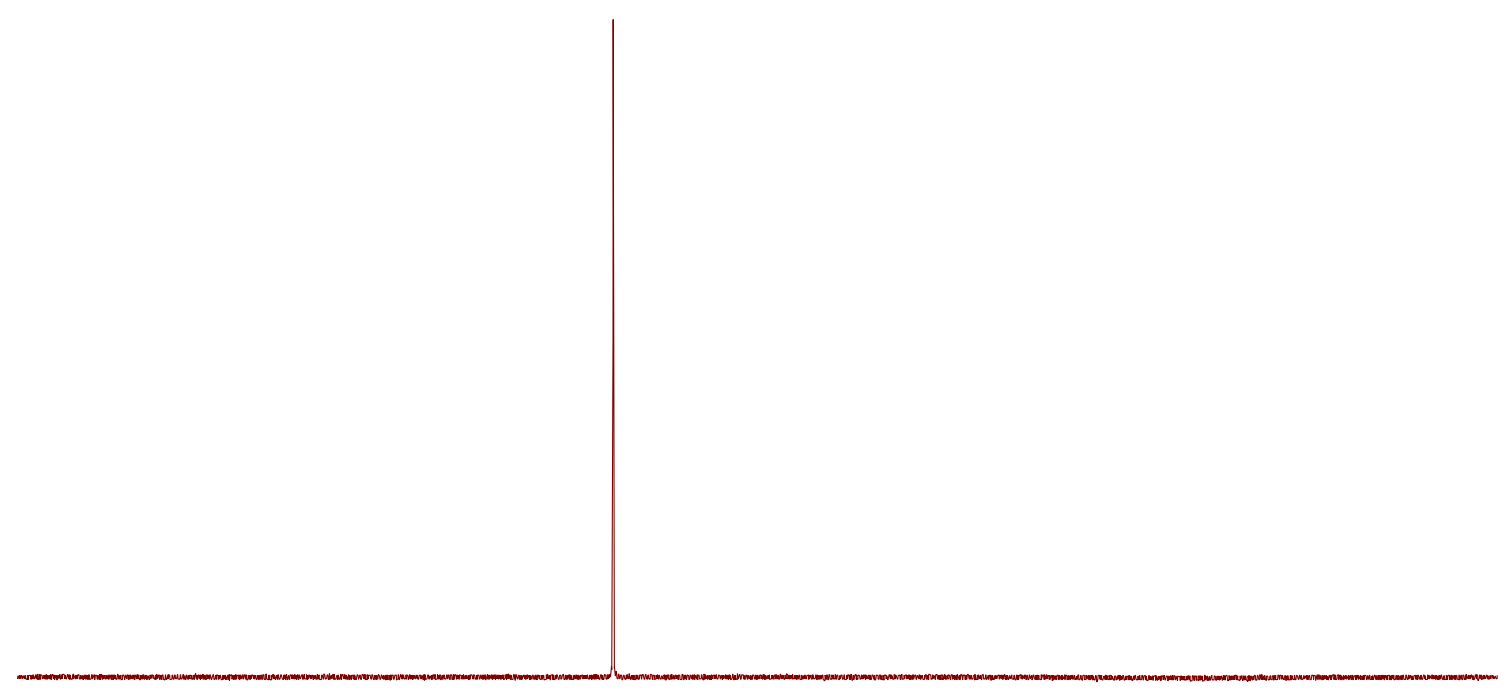

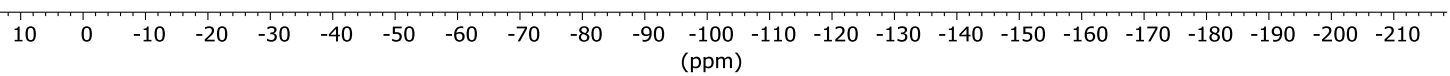

Figure S76. ${ }^{19} \mathrm{~F}$ NMR spectrum (376.49 MHz, $\left.\mathrm{CD}_{3} \mathrm{OD}\right)$ of $\mathbf{2 b}^{\prime} \cdot$ TFA. 
Column: $\quad$ Phenomenex Gemini $5 \mu(2.0 \times 100 \mathrm{~mm})$.

Detection: $\quad \mathrm{UV}$ at $220 \mathrm{~nm}$.

Solvent: $\quad \mathrm{H}_{2} \mathrm{O}, 0.0025 \% \mathrm{TFA}(\mathrm{A}) ; \mathrm{MeOH}(\mathrm{B})$.

Method: $\quad 0 \% \mathrm{~B}(0.0 \rightarrow 3.0 \mathrm{~min}), 11 \rightarrow 16 \% \mathrm{~B}(3.0 \rightarrow 18.0 \mathrm{~min}), 0 \% \mathrm{~B}(18.0 \rightarrow 23.0 \mathrm{~min})$.

Flow rate: $\quad 0.2 \mathrm{~mL} / \mathrm{min}$.

(a) Before semi-preparative HPLC (2b-TFA/2 $\left.\mathbf{b}^{\prime} \cdot \mathrm{TFA}\right)$.

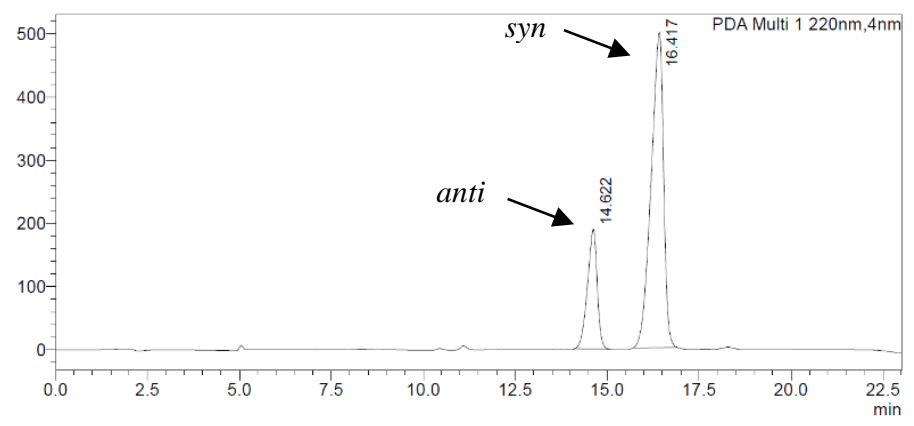

\begin{tabular}{|c|c|c|}
\hline$R_{\mathrm{t}}(\min )$ & Area $(\%)$ & Attribution \\
\hline 14.622 & 22.928 & $\mathbf{2} \mathbf{b}^{\prime} \cdot \mathrm{TFA}$ \\
\hline 16.417 & 77.072 & $\mathbf{2 b} \cdot \mathrm{TFA}$ \\
\hline
\end{tabular}

(b) After semi-preparative HPLC (2b-TFA).

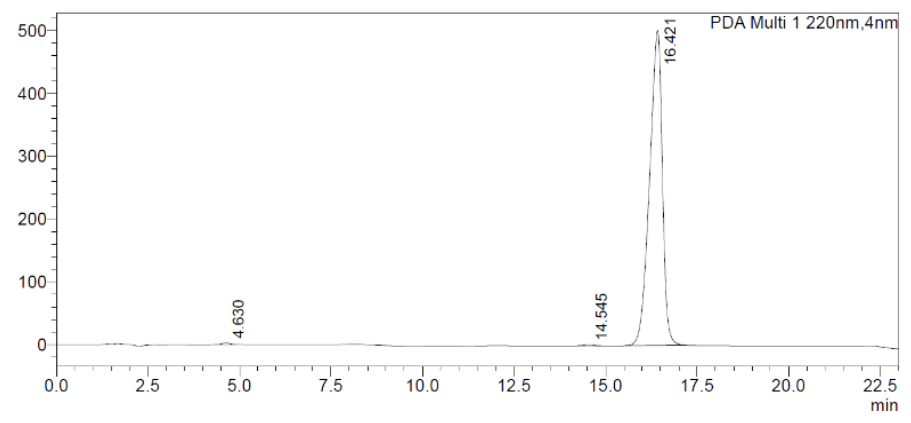

\begin{tabular}{rrc}
$\boldsymbol{R}_{\mathbf{t}}(\mathbf{m i n})$ & Area $(\boldsymbol{\%})$ & Attribution \\
4.630 & 0.301 & Impurity \\
14.545 & 0.246 & $\mathbf{2 b ^ { \prime }} \cdot$ TFA \\
16.421 & 99.453 & $\mathbf{2 b} \cdot$ TFA \\
\hline
\end{tabular}

(c) After semi-preparative HPLC (2b'·TFA).

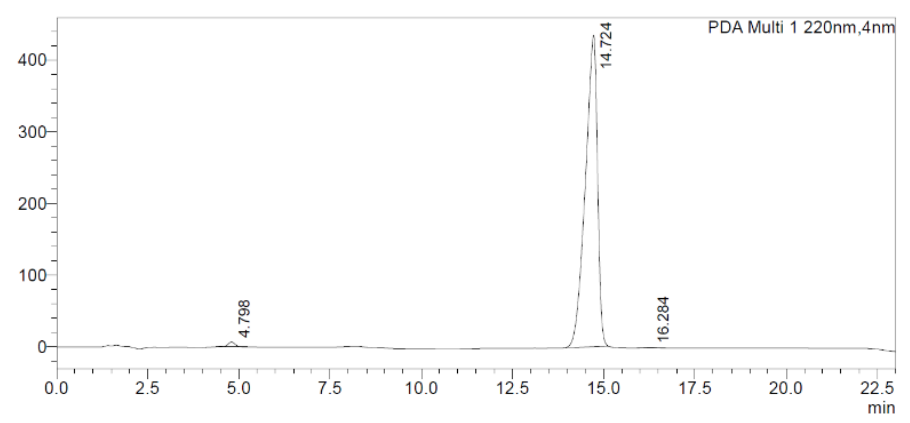

\begin{tabular}{rrc}
$\boldsymbol{R}_{\mathbf{t}}(\mathbf{m i n})$ & Area $(\boldsymbol{\%})$ & Attribution \\
4.798 & 1.026 & Impurity \\
14.724 & 98.770 & $\mathbf{2 b ^ { \prime }} \cdot \mathrm{TFA}$ \\
16.284 & 0.205 & $\mathbf{2 b} \cdot \mathrm{TFA}$ \\
\hline
\end{tabular}

Figure S77. HPLC chromatograms of $\mathbf{2} \mathbf{b}$. TFA and $\mathbf{2} \mathbf{b}^{\prime}$. TFA. 


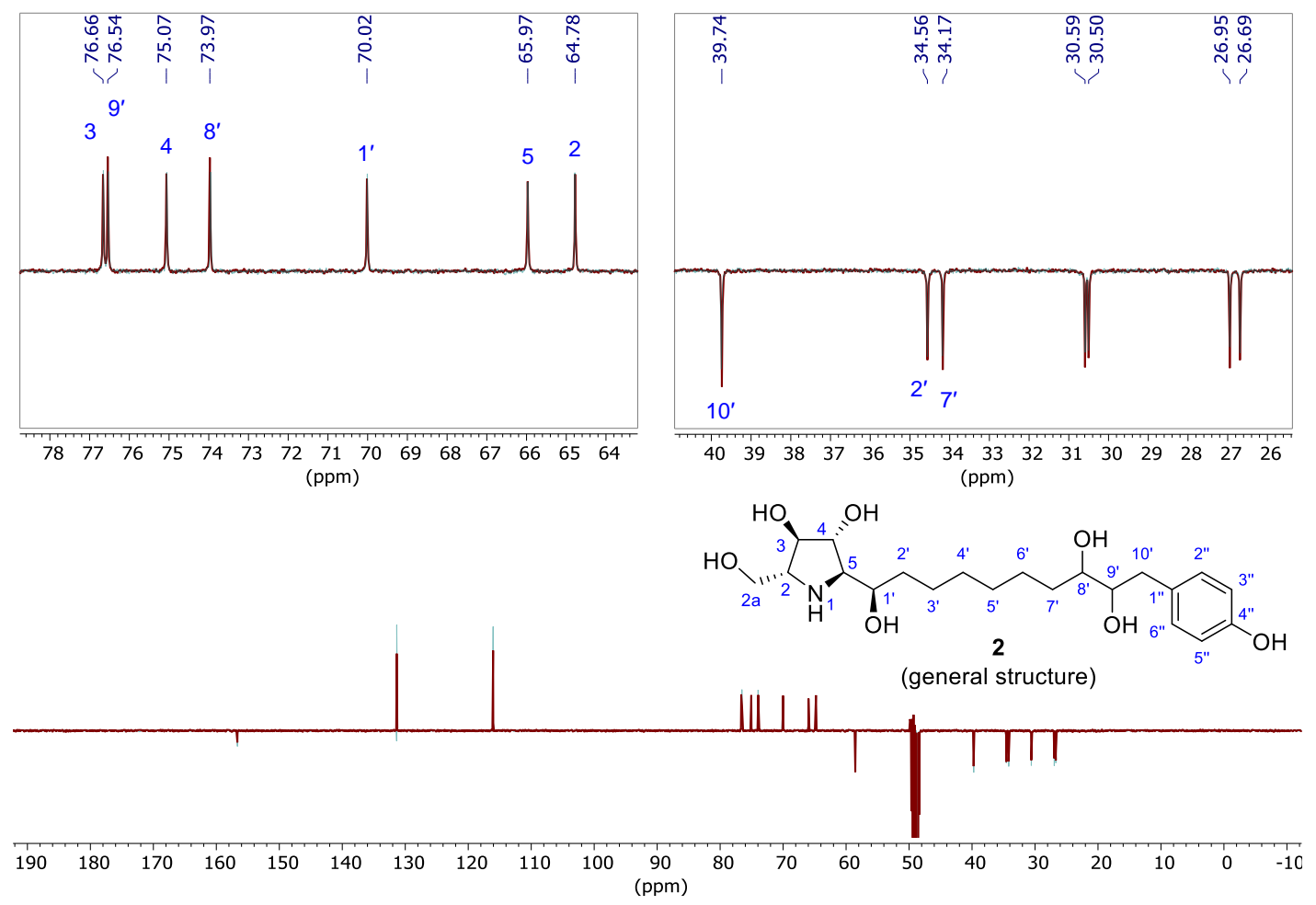

Figure S78. Superimposed DEPTQ spectra $\left(400.16 \mathrm{MHz}, \mathrm{CD}_{3} \mathrm{OD}\right)$ of $\mathbf{2 b} \cdot \mathrm{TFA}$ (bold, maroon) and 2a.TFA (teal).
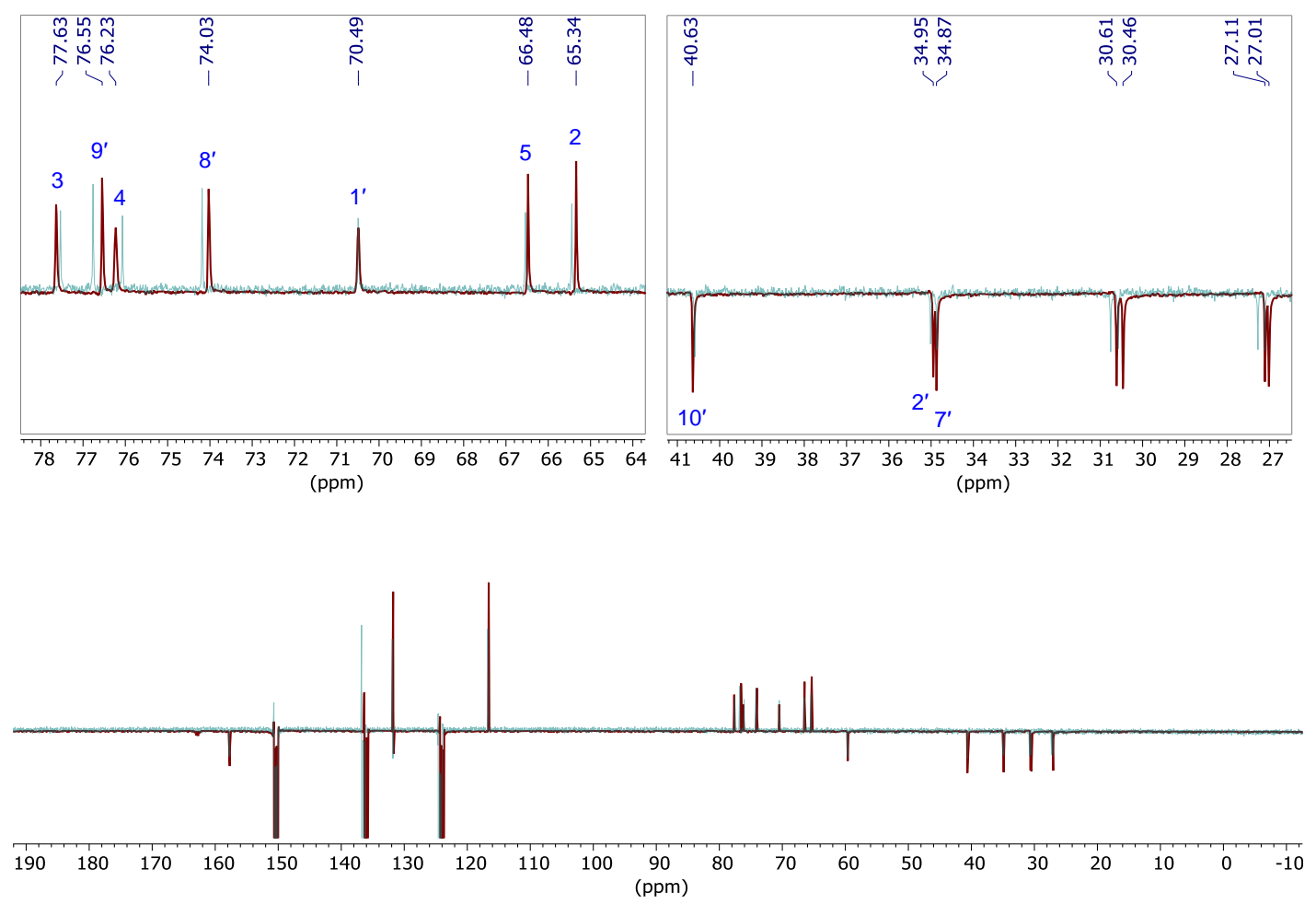

Figure S79. Superimposed DEPTQ spectra (125.67 MHz, $\left.\mathrm{C}_{5} \mathrm{D}_{5} \mathrm{~N}\right)$ of $\mathbf{2 b}$. TFA (bold, maroon) and 2a.TFA (teal). 


\section{Notes and References}

(1) Gossan, D. P. A.; Alabdul Magid, A.; Kouassi-Yao, P. A.; Behr, J.-B.; Ahibo, A. C.; Djakouré, L. A.; Harakat, D.; Voutquenne-Nazabadioko, L. Glycosidase inhibitors from the roots of Glyphaea brevis. Phytochemistry 2015, 109, 76.

(2) Godin, G.; Compain, P.; Martin, O. R.; Ikeda, K.; Yu, L.; Asano, N. $\alpha$-1-C-Alkyl-1-deoxynojirimycin derivatives as potent and selective inhibitors of intestinal isomaltase: remarkable effect of the alkyl chain length on glycosidase inhibitory profile. Bioorg. Med. Chem. Lett. 2004, 14, 5991.

(3) Wennekes, T.; van den Berg, R. J. B. H. N.; Boltje, T. J.; Donker-Koopman, W. E.; Kuijper, B.; van der Marel, G. A.; Strijland, A.; Verhagen, C. P.; Aerts, J. M. F. G.; Overkleeft, H. S. Synthesis and Evaluation of Lipophilic Aza-C-glycosides as Inhibitors of Glucosylceramide Metabolism. Eur. J. Org. Chem. 2010, 1258.

(4) Kato, A.; Hayashi, E.; Miyauchi, S.; Adachi, I.; Imahori, T.; Natori, Y.; Yoshimura, Y.; Nash, R. J.; Shimaoka, H.; Nakagome, I.; Koseki, J.; Hirono, S.; Takahata, H. $\alpha$-1-C-Butyl-1,4-dideoxy-1,4imino-L-arabinitol as a Second-Generation Iminosugar-Based Oral $\alpha$-Glucosidase Inhibitor for Improving Postprandial Hyperglycemia. J. Med. Chem. 2012, 55, 10347.

(5) Asano, N.; Nishida, M.; Miyauchi, M.; Ikeda, K.; Yamamoto, M.; Kizu, H.; Kameda, Y.; Watson, A. A.; Nash, R. J.; Fleet, G. W. J. Polyhydroxylated pyrrolidine and piperidine alkaloids from Adenophora triphylla var. japonica (Campanulaceae). Phytochemistry 2000, 53, 379.

(6) Kato, A.; Yamashita, Y.; Nakagawa, S.; Koike, Y.; Adachi, I.; Hollinshead, J.; Nash, R. J.; Ikeda, K.; Asano, N. 2,5-Dideoxy-2,5-imino-D-altritol as a new class of pharmacological chaperone for Fabry disease. Bioorg. Med. Chem. 2010, 18, 3790.

(7) Asano, N.; Yasuda, K.; Kizu, H.; Kato, A.; Fan, J.-Q.; Nash, R. J.; Fleet, G. W. J.; Molyneux, R. J. Novel $\alpha$-L-fucosidase inhibitors from the bark of Angylocalyx pynaertii (Leguminosae). Eur. J. Biochem. 2001, 268, 35.

(8) Garegg, P. J.; Samuelsson, B. Conversion of Vicinal Diols into Olefins using Triphenylphosphine and Triiodoimidazole. Synthesis 1979, 813.

(9) Rössler, S. L.; Schreib, B. S.; Ginterseder, M.; Hamilton, J. Y.; Carreira, E. M. Total Synthesis and Stereochemical Assignment of (+)-Broussonetine H. Org. Lett. 2017, 19, 5533.

(10) Carmona, A. T.; Whigtman, R. H.; Robina, I.; Vogel, P. Synthesis and Glycosidase Inhibitory Activity of 7-Deoxycasuarine. Helv. Chim. Acta 2003, 86, 3066.

(11) Martella, D.; D’Adamio, G.; Parmeggiani, C.; Cardona, F.; Moreno-Clavijo, E.; Robina, I.; Goti, A. Cycloadditions of Sugar-Derived Nitrones Targeting Polyhydroxylated Indolizidines. Eur. J. Org. Chem. 2016, 1588.

(12) The ${ }^{13} \mathrm{C}$ NMR spectrum of $(E)$-S4 in ref. [10] appears to be mis-referenced by approx. 5.7 ppm by comparison of the reported $\mathrm{Me}_{3} \mathrm{Si}$ resonance with that of the (Z)-isomer.

(13) Tsou, E.-L.; Yeh, Y.-T.; Liang, R.-H.; Cheng, W.-C. A convenient approach toward the synthesis of enantiopure isomers of DMDP and ADMDP. Tetrahedron 2009, 65, 93.

(14) Li, Y.-X.; Huang, M.-H.; Yamashita, Y.; Kato, A.; Jia, Y.-M.; Wang, W.-B.; Fleet, G. W. J.; Nash, R. J.; Yu, C.-Y. L-DMDP, L-homoDMDP and their C-3 fluorinated derivatives: synthesis and glycosidase-inhibition. Org. Biomol. Chem. 2011, 9, 3405.

(15) Commercial $m$-CPBA (70\%) was purified by dissolving in $\mathrm{Et}_{2} \mathrm{O}$, washing with a $\mathrm{NaOH} / \mathrm{KH}_{2} \mathrm{PO}_{4}$ buffer (pH 7.5), drying over $\mathrm{MgSO}_{4}$, and concentrating. Purity was measured by ${ }^{1} \mathrm{H} \mathrm{NMR}$ analysis, and the reagent was stored in a plastic container at $-20^{\circ} \mathrm{C}$. 
(16) Measured using integration of $\mathrm{CH}_{2} \mathrm{Cbz}(\mathbf{1 1 b})$ and $=\mathrm{CH}_{2}$ (11b and $\left.\mathbf{6 b}\right)$, accounting for differences in molecular mass, as fractions of the isolated yield.

(17) Jo, H.; Choi, M.; Viji, M.; Lee, Y. H.; Kwak, Y.-S.; Lee, K.; Choi, N. S.; Lee, Y.-J.; Lee, H.; Hong, J. T.; Lee, M. K.; Jung, J.-K. Concise Synthesis of Broussonone A. Molecules 2015, 20, 15966.

(18) Denton, R. M.; Scragg, J. T.; Saska, J. A concise synthesis of 4'-O-methyl honokiol. Tetrahedron Lett. 2011, 52, 2554.

(19) Devi, R.; Das, S. K. Combining spiro-fused cyclohexadienone - tetrahydrofuran ring system with glycine: Asymmetric synthesis of a new class of $\alpha$-amino acid derivatives. Tetrahedron Lett. 2018, $59,2281$.

(20) Uchiyama, M.; Furuyama, T.; Kobayashi, M.; Matsumoto, Y.; Tanaka, K. Toward a ProtectingGroup-Free Halogen-Metal Exchange Reaction: Practical, Chemoselective Metalation of Functionalized Aromatic Halides Using Dianion-type Zincate, ${ }^{t} \mathrm{Bu}_{4} \mathrm{ZnLi}_{2} . J$. Am. Chem. Soc. 2006, 128, 8404. 